\title{
PARTIAL LINEAR SPACES WITH A RANK 3 AFFINE PRIMITIVE GROUP OF AUTOMORPHISMS
}

\author{
JOHN BAMBERG, ALICE DEVILLERS, JOANNA B. FAWCETT, CHERYL E. PRAEGER
}

\begin{abstract}
A partial linear space is a pair $(\mathcal{P}, \mathcal{L})$ where $\mathcal{P}$ is a non-empty set of points and $\mathcal{L}$ is a collection of subsets of $\mathcal{P}$ called lines such that any two distinct points are contained in at most one line, and every line contains at least two points. A partial linear space is proper when it is not a linear space or a graph. A group of automorphisms $G$ of a proper partial linear space acts transitively on ordered pairs of distinct collinear points and ordered pairs of distinct non-collinear points precisely when $G$ is transitive of rank 3 on points. In this paper, we classify the finite proper partial linear spaces that admit rank 3 affine primitive automorphism groups, except for certain families of small groups, including subgroups of $\mathrm{A}_{1}(q)$. Up to these exceptions, this completes the classification of the finite proper partial linear spaces admitting rank 3 primitive automorphism groups. We also provide a more detailed version of the classification of the rank 3 affine primitive permutation groups, which may be of independent interest.
\end{abstract}

\section{INTRODUCTION}

Partial linear spaces are a class of incidence structures that generalise both graphs and linear spaces. Examples include polar spaces and generalised quadrangles. Specifically, a partial linear space $\mathcal{S}$ is a pair $(\mathcal{P}, \mathcal{L})$, where $\mathcal{P}$ is a non-empty set of points and $\mathcal{L}$ is a collection of subsets of $\mathcal{P}$ called lines such that any two distinct points are contained in at most one line, and every line contains at least two points. In this paper, the set $\mathcal{P}$ will always be finite. A partial linear space $\mathcal{S}$ is a linear space when any two distinct points are contained in exactly one line, and a graph when every line contains exactly two points. A partial linear space is proper when it is neither a linear space nor a graph; in particular, every proper partial linear space contains at least one line. If every line of $\mathcal{S}$ contains exactly $k$ points, then we say that $\mathcal{S}$ has line-size $k$; dually, if every point of $\mathcal{S}$ lies on exactly $\ell$ lines, then we say that $\mathcal{S}$ has point-size $\ell$. The automorphism group $\operatorname{Aut}(\mathcal{S})$ of $\mathcal{S}$ consists of those permutations of $\mathcal{P}$ that preserve $\mathcal{L}$.

Highly symmetric linear spaces have been studied extensively. For example, Kantor [36] classified the 2-transitive linear spaces, in which some automorphism group acts transitively on ordered pairs of distinct points; these include the projective space $\mathrm{PG}_{n}(q)$ and the affine space $\mathrm{AG}_{n}(q)$. More generally, the flag-transitive linear spaces $\mathcal{S}$-in which some automorphism group $G$ acts transitively on point-line incident pairs - have also been classified [9], except for the case where $\mathcal{S}$ has $q$ points and $G \leqslant \operatorname{A\Gamma L}_{1}(q)$ for some prime power $q$. The most natural way to generalise the concept of 2-transitivity to arbitrary partial linear spaces is to consider those partial linear spaces for which some automorphism group acts transitively on ordered pairs of distinct collinear points, as well as ordered pairs of distinct non-collinear points. Such partial linear spaces are flag-transitive, and when they have non-empty line sets and are not linear spaces, they are precisely the partial linear spaces $\mathcal{S}$ for which $\operatorname{Aut}(\mathcal{S})$ is transitive of rank 3 on points (see Lemma 2.4 and Remark 2.5).

A permutation group $G$ acting on a finite set $\Omega$ has rank $r$ when $G$ is transitive on $\Omega$ and has $r$ orbits on $\Omega \times \Omega$; we also say that $G$ is primitive if it is transitive on $\Omega$ and there are no non-trivial $G$-invariant equivalence relations on $\Omega$. Using the classification of the finite simple groups (CFSG), the primitive permutation groups of rank 3 have been classified (see [42] for

2010 Mathematics Subject Classification. 51E30, 05E18, 20B15, 05B25, $20 \mathrm{~B} 25$.

Key words and phrases. partial linear space, hypergraph, flag-transitive, rank 3 affine primitive group.

We sincerely thank the anonymous referees for their helpful and insightful comments. This work forms part of the Australian Research Council Discovery Project grant DP130100106 of the first, second and fourth authors. The third author was supported by this same grant, the London Mathematical Society and the European Union's Horizon 2020 research and innovation programme under the Marie Skłodowska-Curie grant agreement No. 746889". 
references), and as an immediate consequence of this classification, the graphs with a transitive automorphism group of rank 3 are known. However, we cannot make such a deduction for proper partial linear spaces, for the lines of such geometries are not necessarily determined by their collinearity relations. We therefore wish to classify the proper partial linear spaces with a rank 3 automorphism group $G$, and we will focus on the case where $G$ is primitive (see Remark 2.10 for some comments on the imprimitive case). The primitive permutation groups of rank 3 are of almost simple, grid or affine type (see Proposition 2.21), and Devillers has classified the partial linear spaces with a rank 3 primitive automorphism group of almost simple [16] or grid type [17], so it remains to consider those of affine type.

A primitive permutation group $G$ is affine when the socle of $G$ is (the additive group of) a vector space $V:=V_{d}(p)$ for some $d \geqslant 1$ and prime $p$, in which case we may view $G$ as a subgroup of $\mathrm{AGL}_{d}(p)$ acting on $V$. Moreover, we may view $V$ as the translation group of $\mathrm{AGL}_{d}(p)$, in which case $G=V: G_{0}$, where $G_{0}$ denotes the stabiliser of the zero vector in $V$, and $G_{0}$ is an irreducible subgroup of $\mathrm{GL}_{d}(p)$. Note that if $G_{0} \leqslant \Gamma \mathrm{L}_{a}(r)$ where $r^{a}=p^{d}$, then we may view $V$ as a vector space $V_{a}(r)$. The affine primitive permutation groups of rank 3 were classified by Foulser [24] in the soluble case and Liebeck [39] in general, and we provide a more detailed version of this classification in this paper (see Theorem 3.1). If $G$ is such a permutation group, then $G_{0}$ has two orbits on $V^{*}:=V \backslash\{0\}$, and if $\mathcal{S}$ is a partial linear space with $G \leqslant \operatorname{Aut}(\mathcal{S})$, then we may identify the points of $\mathcal{S}$ with $V$, in which case the set of vectors in $V$ that are collinear with 0 , denoted by $\mathcal{S}(0)$, is one of the two orbits of $G_{0}$ on $V^{*}$.

Before we state the main theorem of this paper, we first describe some infinite families of examples that arise. Throughout these examples, $p$ is a prime and $d$ is a positive integer. See $\S 2$ for any unexplained terminology. We will see that most of the proper partial linear spaces admitting rank 3 affine primitive automorphism groups have the form of Example 1.1.

Example 1.1. Let $(G, a, r)$ satisfy the following properties: $G$ is an affine primitive permutation group of rank 3 with socle $V:=V_{d}(p)$ such that $G_{0} \leqslant \Gamma L_{a}(r)$ and $G_{0}$ has two orbits $\Delta_{1}$ and $\Delta_{2}$ on the points of $\mathrm{PG}_{a-1}(r)$, where $r^{a}=p^{d}, a \geqslant 2$ and $r>2$. Let $\mathcal{L}_{i}:=\left\{\langle u\rangle+v:\langle u\rangle \in \Delta_{i}, v \in V\right\}$ for $i \in\{1,2\}$. Then $\mathcal{S}_{i}:=\left(V, \mathcal{L}_{i}\right)$ is a proper partial linear space such that $G \leqslant \operatorname{Aut}\left(\mathcal{S}_{i}\right)$ for $i \in\{1,2\}$. Note that $\left(V, \mathcal{L}_{1} \cup \mathcal{L}_{2}\right)$ is the linear space $\mathrm{AG}_{a}(r)$, and $G$ has orbits $\mathcal{L}_{1}$ and $\mathcal{L}_{2}$ on the lines of $\mathrm{AG}_{a}(r)$. Geometrically, if we embed $\mathrm{PG}_{a-1}(r)$ as a hyperplane $\Pi$ in $\mathrm{PG}_{a}(r)$ and view $V$ as the set of points in $\mathrm{AG}_{a}(r)$ (i.e., the set of points in $\mathrm{PG}_{a}(r)$ that are not in $\Pi$ ), then $\mathcal{L}_{i}$ is the set of affine lines of $\mathrm{AG}_{a}(r)$ whose completions meet $\Pi$ in a point of $\Delta_{i}$. For most rank 3 affine primitive permutation groups $G$, there are various pairs $(a, r)$ such that $G_{0} \leqslant \Gamma \mathrm{L}_{a}(r)$ and $G_{0}$ has two orbits on $\mathrm{PG}_{a-1}(r)$; see Hypothesis 3.6 and Corollary 3.7 (and Theorem 3.1).

Example 1.2. Let $U:=V_{2}(q)$ and $W:=V_{m}(q)$ where $q^{2 m}=p^{d}$ and $m \geqslant 2$. Let $V:=U \otimes W$. Let $\Sigma_{U}:=\left\{U \otimes w: w \in W^{*}\right\}$ and $\Sigma_{W}:=\left\{u \otimes W: u \in U^{*}\right\}$. For $X \in\{U, W\}$, let $\mathcal{L}_{X}:=\{Y+v:$ $\left.Y \in \Sigma_{X}, v \in V\right\}$. Then $\mathcal{S}_{X}:=\left(V, \mathcal{L}_{X}\right)$ is a proper partial linear space, and $\mathcal{S}_{U} \simeq \mathcal{S}_{W}$ when $m=2$. Geometrically, if we embed $\mathrm{PG}_{2 m-1}(q)$ as a hyperplane $\Pi$ in $\mathrm{PG}_{2 m}(q)$ and view $V$ as the set of points in $\mathrm{AG}_{2 m}(q)$ (i.e., the set of points in $\mathrm{PG}_{2 m}(q)$ that are not in $\Pi$ ), then for $(X, n) \in$ $\{(U, 2),(W, m)\}$, we may view $\Sigma_{X}$ as a set of projective $(n-1)$-subspaces of $\Pi$, whence $\mathcal{L}_{X}$ is the set of affine $n$-subspaces of $\mathrm{AG}_{2 m}(q)$ whose completions meet $\Pi$ in an element of $\Sigma_{X}$. For $\mathcal{S}_{U}$, the line-size is $q^{2}$ and the point-size is $\left(q^{m}-1\right) /(q-1)$, while for $\mathcal{S}_{W}$, the line-size is $q^{m}$ and the point-size is $q+1$. By Proposition 10.10, $\operatorname{Aut}\left(\mathcal{S}_{U}\right)=\operatorname{Aut}\left(\mathcal{S}_{W}\right)=V:\left(\mathrm{GL}_{2}(q) \circ \mathrm{GL}_{m}(q)\right): \operatorname{Aut}\left(\mathbb{F}_{q}\right)$, a rank 3 affine primitive group. See $\S 10$ for more details.

Example 1.3. Let $d=2 n$ where $p^{n} \neq 2$. The $p^{n} \times p^{n}$ grid is a proper partial linear space with point set $V:=V_{n}(p) \oplus V_{n}(p)$ whose line set is the union of $\left\{\left\{(v, w): v \in V_{n}(p)\right\}: w \in V_{n}(p)\right\}$ and $\left\{\left\{(w, v): v \in V_{n}(p)\right\}: w \in V_{n}(p)\right\}$. The $p^{n} \times p^{n}$ grid has line-size $p^{n}$ and point-size 2, and its full automorphism group is $S_{p^{n}}\left\{S_{2}\right.$, which contains the rank 3 affine primitive group $V:\left(\operatorname{GL}_{n}(p) \imath S_{2}\right)$. See $\S 13$ for more details.

Now we state the main result of this paper. 
Theorem 1.4. Let $\mathcal{S}$ be a finite proper partial linear space, and let $G \leqslant \operatorname{Aut}(\mathcal{S})$ such that $G$ is an affine primitive permutation group of rank 3 with socle $V:=V_{d}(p)$ where $d \geqslant 1$ and $p$ is prime. Then one of the following holds.

(i) $\mathcal{S}$ is isomorphic to a partial linear space from Example 1.1 with respect to a triple $(H, a, r)$ satisfying Hypothesis 3.6 such that $H$ is primitive with socle $V$ and $r^{a}=p^{d}$.

(ii) $\mathcal{S}$ is described in Examples 1.2 or 1.3 .

(iii) $\mathcal{S}$ is described in Table 1 where $k$ and $\ell$ are the line- and point-size of $\mathcal{S}$, respectively, and $\operatorname{Aut}(\mathcal{S})=p^{d}: \operatorname{Aut}(\mathcal{S})_{0}$.

(iv) One of the following holds.

(a) $G_{0} \leqslant \Gamma L_{1}\left(p^{d}\right)$.

(b) $V=V_{n}(p) \oplus V_{n}(p)$ and $G_{0} \leqslant \Gamma L_{1}\left(p^{n}\right)$ ᄀ $S_{2}$ where $\mathcal{S}(0)=V_{n}(p)^{*} \times V_{n}(p)^{*}$.

(c) $V=V_{2}\left(q^{3}\right)$ and $\mathrm{SL}_{2}(q) \unlhd G_{0} \leqslant \Gamma L_{2}\left(q^{3}\right)$ where $|\mathcal{S}(0)|=q\left(q^{3}-1\right)\left(q^{2}-1\right)$.

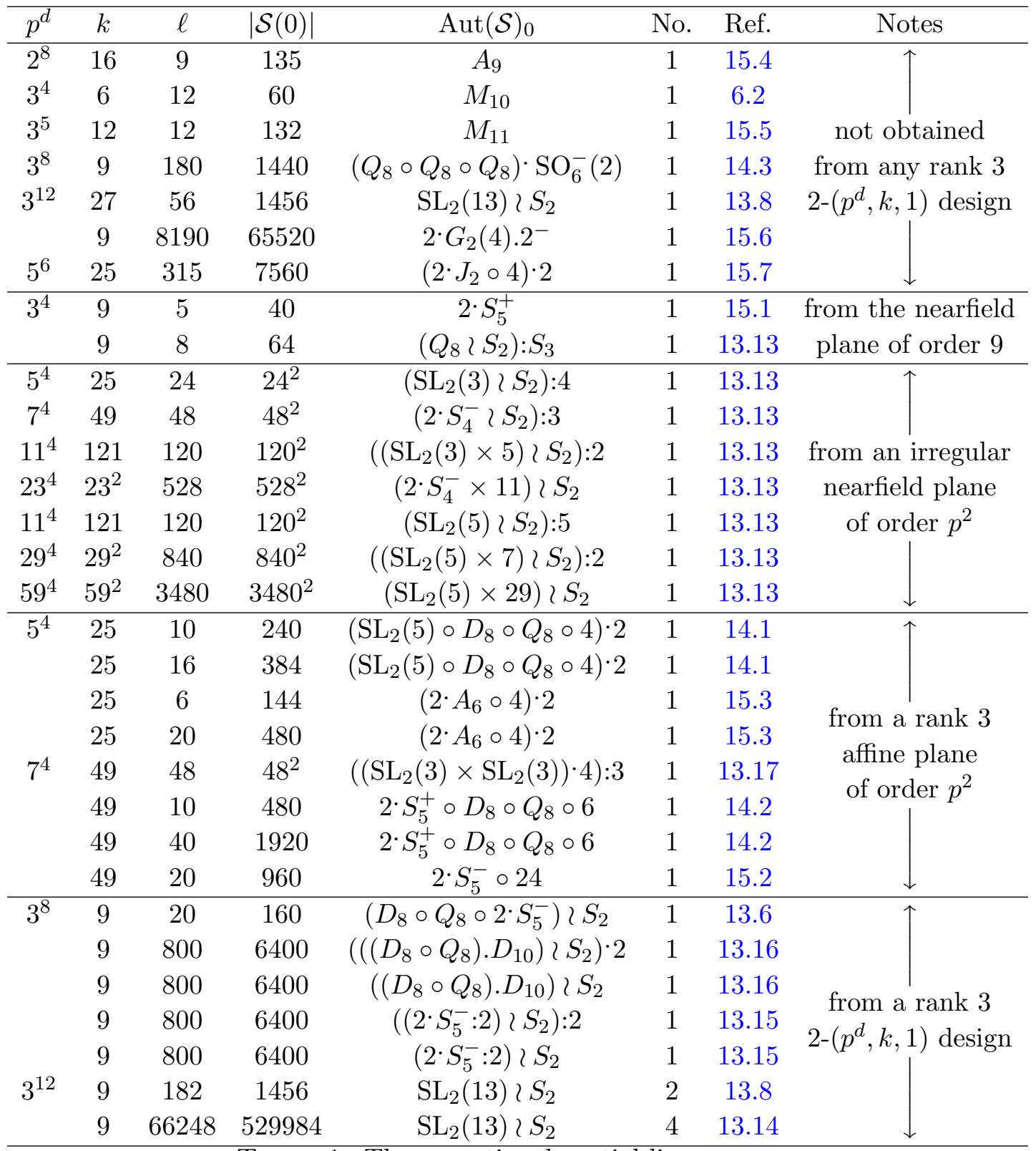

TABLE 1. The exceptional partial linear spaces 
For each exceptional partial linear space $\mathcal{S}$ in Table $1, \operatorname{Aut}(\mathcal{S})$ is an affine primitive group and $\operatorname{Aut}(\mathcal{S})_{0}$, the stabiliser in $\operatorname{Aut}(\mathcal{S})$ of the 0 vector, is an irreducible subgroup of $\mathrm{GL}_{d}(p)$. We also list the following in Table 1: the number (No.) of partial linear spaces that satisfy the given parameters up to isomorphism; a reference (Ref.) for the definition of the partial linear space and its automorphism group; and some additional notes that will be explained in Remark 1.5. Observe that $\mathcal{S}$ has $p^{d} \ell / k$ lines and that $|\mathcal{S}(0)|=\ell(k-1)$ (see Lemma 2.8).

We caution the reader that there are affine primitive permutation groups $G$ of rank 3 and partial linear spaces $\mathcal{S}$ with $G \leqslant \operatorname{Aut}(\mathcal{S})$ such that $\mathcal{S}$ satisfies the conditions of Theorem 1.4(i) with respect to some triple $(H, a, r)$ but not $(G, a, r)$ (see Remark 13.2).

Remark 1.5. One way of constructing a partial linear space is to remove lines from a linear space, as we did in Example 1.1 with the linear space $\mathrm{AG}_{a}(r)$. In particular, for a rank 3 permutation group $G$ on $\mathcal{P}$, if $\mathcal{S}:=(\mathcal{P}, \mathcal{L})$ is a linear space with at least three points on every line such that $G \leqslant \operatorname{Aut}(\mathcal{S})$ and $G$ has exactly two orbits $\mathcal{L}_{1}$ and $\mathcal{L}_{2}$ on $\mathcal{L}$, then $\left(\mathcal{P}, \mathcal{L}_{1}\right)$ and $\left(\mathcal{P}, \mathcal{L}_{2}\right)$ are proper partial linear spaces that admit $G$ and have disjoint collinearity relations; in fact, the converse of this statement also holds (see Lemma 2.11). Observe that $\mathcal{S}$ is a $2-(v, k, 1)$ design - that is, a linear space with $v$ points and line-size $k$-precisely when $\left(\mathcal{P}, \mathcal{L}_{1}\right)$ and $\left(\mathcal{P}, \mathcal{L}_{2}\right)$ both have line-size $k$. Those $2-(v, k, 1)$ designs admitting a rank 3 automorphism group $G$ with two orbits on lines have been studied in the special case of affine planes (e.g., [3]) and in general when $G$ is an affine primitive group $[4,46]$. For each partial linear space $\mathcal{S}$ in Table 1 , we state whether $\mathcal{S}$ can be obtained from a $2-(v, k, 1)$ design using a rank 3 group as above; when this design is an affine plane (i.e., when $v=k^{2}$ ), we state this instead, and when this affine plane is well known, we give its name. More details may be found at the given reference or in $\S 16$.

It therefore follows from Theorem 1.4 that there are proper partial linear spaces with rank 3 affine primitive automorphism groups that cannot be obtained from any $2-(v, k, 1)$ design using a rank 3 group as described in Remark 1.5. In fact, there are infinitely many such structures: we prove that the partial linear space $\mathcal{S}_{W}$ from Example 1.2 cannot be obtained from a $2-(v, k, 1)$ design using any rank 3 group for $m \geqslant 4$ and $(m, q) \neq(5,2)$; see Proposition 18.1. However, if $\mathcal{S}$ is $\mathcal{S}_{U}$ from Example 1.2 for $m \geqslant 2$ (respectively, $\mathcal{S}_{W}$ for $m=3$ ), then $\mathcal{S}$ can be obtained from $\mathrm{AG}_{m}\left(q^{2}\right)$ (respectively, $\mathrm{AG}_{2}\left(q^{3}\right)$ ) using a rank 3 affine primitive subgroup $G$ of $\operatorname{Aut}(\mathcal{S})($ see $\S 18)$; in other words, $\mathcal{S}$ is described in Example 1.1 with respect to the triple $\left(G, m, q^{2}\right)$ (respectively, $\left.\left(G, 2, q^{3}\right)\right)$, but we choose not to omit $\mathcal{S}$ from Example 1.2 because $\operatorname{Aut}(\mathcal{S})$ is itself a rank 3 affine primitive group. Similarly, if $\mathcal{S}$ is the $p^{n} \times p^{n}$ grid of Example 1.3, then $\mathcal{S}$ can be obtained from the affine plane $\mathrm{AG}_{2}\left(p^{n}\right)$ using the rank 3 affine primitive group $V:\left(\mathrm{GL}_{1}\left(p^{n}\right) \gtrless S_{2}\right)$, but this group is considerably smaller than $\operatorname{Aut}(\mathcal{S})$.

Remark 1.6. We are unable to classify the partial linear spaces that satisfy the conditions of Theorem 1.4(iv). In fact, the groups of Theorem 1.4(iv)(a) were also omitted from the classification of the flag-transitive linear spaces [9]. There are partial linear spaces from Example 1.1 for which $G$ satisfies the conditions of Theorem 1.4(iv)(a); see Example 4.5 for more details. There are also partial linear spaces $\mathcal{S}$ for which $G$ and $\mathcal{S}(0)$ satisfy the conditions of Theorem 1.4(iv)(b); see Examples 13.12 and 13.13. We completely classify the proper partial linear spaces $\mathcal{S}$ for which $G$ and $\mathcal{S}(0)$ satisfy the conditions of Theorem 1.4(iv)(c) under the extra assumption that $\mathrm{GL}_{2}(q) \circ Z\left(\mathrm{GL}_{2}\left(q^{3}\right)\right) \leqslant G_{0}$ (see Proposition 12.2); several infinite families arise, including some from Example 1.1. However, we believe that the situation is much more complicated in general. We illustrate this by providing a complete classification of the partial linear spaces that arise when $q=4$ (see Example 12.8).

All of the partial linear spaces of Examples 1.1, 1.2 and 1.3 have the property that their lines are affine subspaces of $V_{d}(p)$. Moreover, this turns out to be true for all of the partial linear spaces in Table 1 except when $\operatorname{Aut}(\mathcal{S})=3^{4}: M_{10}$ or $3^{5}: M_{11}$, in which case neither partial linear space has this property since the line-size is not a power of $p$. We suspect that these are the 
only two such partial linear spaces, and we prove that any other such partial linear space must satisfy the conditions of Theorem 1.4(iv)(b) as well as the constraints in (iii) below.

Corollary 1.7. Let $\mathcal{S}$ be a finite proper partial linear space, and let $G \leqslant \operatorname{Aut}(\mathcal{S})$ such that $G$ is an affine primitive permutation group of rank 3 with socle $V:=V_{d}(p)$ where $d \geqslant 1$ and $p$ is prime. If the lines of $\mathcal{S}$ are not affine subspaces of $V_{d}(p)$, then one of the following holds.

(i) $p^{d}=3^{4}, \mathcal{S}$ has line-size 6 and $\operatorname{Aut}(\mathcal{S})=3^{4}: M_{10}$.

(ii) $p^{d}=3^{5}, \mathcal{S}$ has line-size 12 and $\operatorname{Aut}(\mathcal{S})=3^{5}: M_{11}$.

(iii) $V=V_{n}(p) \oplus V_{n}(p)$ and $G_{0} \leqslant \Gamma L_{1}\left(p^{n}\right) 2 S_{2}$ where $\mathcal{S}(0)=V_{n}(p)^{*} \times V_{n}(p)^{*}$ and $n \geqslant 2$. Further, all of the following hold for any line $L$ of $\mathcal{S}$ such that $0 \in L$.

(1) The prime $p$ is odd, and $-1 \notin G_{0}$. In particular, $\left|G_{0} \cap Z\left(\mathrm{GL}_{d}(p)\right)\right|$ is odd.

(2) If $k$ is the line-size of $\mathcal{S}$, then $k(k-1)$ divides $\left(p^{n}-1\right)^{2}$, so $k$ is coprime to $p$.

(3) $L \cap\left\{\lambda u: \lambda \in \mathbb{F}_{p}\right\}=\{0, u\}$ for all $u \in L^{*}$.

(4) $L=\left\{\left(v, v^{\alpha}\right): v \in M\right\}$ for some $M \subseteq V_{n}(p)$ and injective map $\alpha: M \rightarrow V_{n}(p)$.

(5) For $g \in G_{0}$, there exists $v \in L$ such that $v^{g} \neq-v$.

(6) There exist $g_{1}, g_{2} \in \Gamma L_{1}\left(p^{n}\right)$ such that $\left(g_{1},-1\right) \in G_{0}$ and $\left(-1, g_{2}\right) \in G_{0}$.

(7) If $H \times K \leqslant G_{0}$ for some $H, K \leqslant \Gamma L_{1}\left(p^{n}\right)$, then $H$ or $K$ is not transitive on $V_{n}(p)^{*}$.

We saw in Example 1.3 that the $p^{n} \times p^{n}$ grid is a proper partial linear space with a rank 3 affine primitive group of automorphisms whose full automorphism group is not affine. Using [49], we prove that this is the only such example.

Theorem 1.8. Let $\mathcal{S}$ be a finite proper partial linear space, and let $G \leqslant \operatorname{Aut}(\mathcal{S})$ such that $G$ is an affine primitive permutation group of rank 3 with socle $V:=V_{d}(p)$ where $d \geqslant 1$ and $p$ is prime. Then one of the following holds.

(i) $\mathcal{S}$ is isomorphic to the $p^{n} \times p^{n}$ grid and $\operatorname{Aut}(\mathcal{S})=S_{p^{n}} \backslash S_{2}$, where $d=2 n$ and $n \geqslant 1$.

(ii) $\operatorname{Aut}(\mathcal{S})$ is an affine primitive permutation group of rank 3 with socle $V$.

This paper is organised as follows. In $\S 2$, we give some preliminaries and prove Theorem 1.8. In $\S 3$, we state and prove a modified version of Liebeck's classification [39] of the affine primitive permutation groups of rank 3 (see Theorem 3.1); in particular, we provide more detailed information about the possible rank 3 groups $G$ when $G_{0}$ is imprimitive or stabilises a tensor product decomposition. We then use Theorem 3.1 to state Hypothesis 3.6. The partial linear spaces of Example 1.1 have a property that we term dependence (see $\S 4$ ), and we prove in $\S 4$ that, under very general assumptions, the dependent partial linear spaces all have the form of Example 1.1; we also provide more details about our strategy for proving Theorem 1.4. In §5-12, we classify the independent proper partial linear spaces for various classes of rank 3 affine primitive groups, as defined by Theorem 3.1, and in $\S 13-15$, we consider the remaining rank 3 groups. In $\S 16$, we prove Theorem 1.4, and in $\S 17$, we prove Corollary 1.7. In $\S 18$, we consider when a partial linear space from Example 1.2 can be obtained from a 2- $(v, k, 1)$ design using a rank 3 group.

\section{Preliminaries}

All groups and incidence structures in this paper are finite, and all group actions are written on the right. Basic terminology and results in permutation group theory or representation theory may be found in [20] or [32], respectively. The notation used to denote the finite simple groups (and their automorphism groups) is consistent with [37]. We use the algebra software MAGMA [6] and GAP [26] for a variety of computations. In particular, we use the GAP package FinInG [2], as well as nauty and Traces [45] underneath the GAP package Grape [52].

This section is organised as follows. In $\S 2.1-2.5$, we review some general notation, definitions and basic results. In $\S 2.6$, we consider some elementary properties of partial linear spaces. In $\S 2.7$, we describe several ways of constructing new partial linear spaces from given ones. In $\S 2.8$, we investigate some properties of partial linear spaces admitting affine automorphism groups. In $\S 2.9$, we describe a family of affine planes called nearfield planes. In $\S 2.10$, we 
state the classifications of the 2-transitive affine groups $[28,30]$ and the linear spaces admitting 2 -transitive affine automorphism groups [36]. In §2.11, we prove Theorem 1.8.

2.1. Group actions. Let $G$ be a group acting on a (finite) set $\Omega$. We denote the orbit of $x \in \Omega$ by $x^{G}$ and the pointwise stabiliser in $G$ of $x \in \Omega$ by $G_{x}$. We denote the setwise and pointwise stabilisers of $X=\left\{x_{1}, \ldots, x_{n}\right\} \subseteq \Omega$ in $G$ by $G_{X}$ and $G_{(X)}=G_{x_{1}, \ldots, x_{n}}$, respectively. We denote the permutation group induced by $G_{X}$ on $X$ by $G_{X}^{X}=G_{X} / G_{(X)}$, and the symmetric group on $\Omega$ by $\operatorname{Sym}(\Omega)$. The degree of $G$ is $|\Omega|$.

A block of $G$ is a non-empty subset $B$ of $\Omega$ such that for each $g \in G$, either $B^{g}=B$ or $B^{g} \cap B=\varnothing$. If $G$ is transitive on $\Omega$, then a block $B$ is non-trivial if it is neither a singleton nor $\Omega$, in which case $\left\{B^{g}: g \in G\right\}$ is a system of imprimitivity for $G$ on $\Omega$, and $G$ is imprimitive; recall from the introduction that $G$ is primitive if no such system of imprimitivity exists. We will use the following observation throughout this paper (see [20, Theorem 1.5A]): if $G$ is transitive on $\Omega$ and $x \in \Omega$, then the set of blocks of $G$ containing $x$ is in one-to-one correspondence with the set of subgroups of $G$ containing $G_{x}$; under this correspondence, a block $B$ containing $x$ is mapped to $G_{B}$, and a subgroup $H$ containing $G_{x}$ is mapped to $x^{H}$.

2.2. Notation and definitions for groups. For groups $G$ and $H$, we denote a split extension of $G$ by $H$ by $G: H$; a non-split extension by $G \cdot H$, an arbitrary extension by $G . H$, and the central product of $G$ and $H$ (with respect to some common central subgroup) by $G \circ H$. For $S \leqslant S_{n}$, we denote the wreath product $G^{n}: S$ by $G \imath S$. We denote the cyclic group of order $n$ by $C_{n}$ or just $n$, the elementary abelian group $C_{p}^{n}$ by $p^{n}$, the dihedral group of order $n$ by $D_{n}$, and the quaternion group by $Q_{8}$. For $H \leqslant G$ and $K \leqslant G$, we denote the centraliser and normaliser of $H$ in $K$ by $C_{K}(H)$ and $N_{K}(H)$, respectively. We denote the centre of $G$ by $Z(G)$ and the derived subgroup of $G$ by $G^{\prime}$.

The socle of a group $G$ is the subgroup generated by the minimal normal subgroups of $G$. The group $G$ is almost simple if its socle is a non-abelian simple group $T$; equivalently, $G$ is almost simple if $T \leqslant G \leqslant \operatorname{Aut}(T)$. The group $G$ is quasisimple if $G$ is perfect (i.e., $G=G^{\prime}$ ) and $G / Z(G)$ is a simple group. A covering group of $G$ is a group $L$ such that $L / Z(L) \simeq G$ and $Z(L) \leqslant L^{\prime}$. The symmetric group $S_{n}$ has two covering groups $2 \cdot S_{n}^{+}$and $2 \cdot S_{n}^{-}$for $n \geqslant 4$, both of which contain the covering group $2 \cdot A_{n}$ of $A_{n}$; in $2 \cdot S_{n}^{+}$, transpositions lift to involutions, whereas in $2 \cdot S_{n}^{-}$, transpositions lift to elements of order 4 . The almost simple group $G_{2}(4) .2$ also has two covering groups $2 \cdot G_{2}(4) .2^{+}$and $2 \cdot G_{2}(4) .2^{-}$, where $2 \cdot G_{2}(4) \cdot 2^{+}$is the group whose character table is given in [14, p.99]. Note that $2 \cdot S_{4}^{+} \simeq \mathrm{GL}_{2}(3), 2 \cdot A_{4} \simeq \mathrm{SL}_{2}(3)$ and $2 \cdot A_{5} \simeq \mathrm{SL}_{2}(5)$.

2.3. Fields, vector spaces and representation theory. Let $q$ be a power of a prime $p$. We denote the finite field of order $q$ by $\mathbb{F}_{q}$ and an $n$-dimensional vector space over $\mathbb{F}_{q}$ by $V_{n}(q)$. If $W \subseteq V_{n}(q)$, then we define $W^{*}:=W \backslash\{0\}$. For a subfield $F$ of $\mathbb{F}_{q}$, we write $\left\langle x_{1}, \ldots, x_{m}\right\rangle_{F}$ for the $F$-span of the vectors $x_{1}, \ldots, x_{m} \in V_{n}(q)$; when $F=\mathbb{F}_{q}$ and the context permits, we omit $F$ from this notation. We write $\sigma_{q}$ for the Frobenius automorphism $x \mapsto x^{p}$ of $\mathbb{F}_{q}$, so that $\operatorname{Aut}\left(\mathbb{F}_{q}\right)=\left\langle\sigma_{q}\right\rangle$, and we adopt the following convention: whenever we write $\Gamma \mathrm{L}_{n}(q)=\mathrm{GL}_{n}(q):\langle\sigma\rangle$, we mean that $\langle\sigma\rangle \simeq\left\langle\sigma_{q}\right\rangle$ and $\Gamma \mathrm{L}_{n}(q)$ acts on $V_{n}(q)$ with respect to some basis $\left\{v_{1}, \ldots, v_{n}\right\}$ such that $\left(\sum_{i=1}^{n} \lambda_{i} v_{i}\right)^{\sigma}=\sum_{i=1}^{n} \lambda_{i}^{\sigma} v_{i}$ for all $\lambda_{i} \in \mathbb{F}_{q}$. Any subgroup of $\Gamma_{n}(q)$ is $\mathbb{F}_{q}$-semilinear, and $g \in \Gamma L_{n}(q)$ is $\sigma$-semilinear when $\sigma \in\left\langle\sigma_{q}\right\rangle$ and $(\lambda v)^{g}=\lambda^{\sigma} v^{g}$ for all $\lambda \in \mathbb{F}_{q}$ and $v \in V_{n}(q)$. We write $\operatorname{diag}\left(\lambda_{1}, \ldots, \lambda_{n}\right)$ for the diagonal $n \times n$ matrix with diagonal entries $\lambda_{1}, \ldots, \lambda_{n}$. Now $Z\left(\mathrm{GL}_{n}(q)\right)=\left\{\operatorname{diag}(\lambda, \ldots, \lambda): \lambda \in \mathbb{F}_{q}^{*}\right\}$, and with a slight abuse of notation, we write $\lambda$ for $\operatorname{diag}(\lambda, \ldots, \lambda)$ and $\mathbb{F}_{q}^{*}$ for $Z\left(\mathrm{GL}_{n}(q)\right)$. We also write $\zeta_{q}$ for a generator of the multiplicative group of $\mathbb{F}_{q}$. Note that -1 denotes the central involution of $\mathrm{GL}_{n}(q)$ when $p$ is odd, but $-1=1$ when $p$ is even.

For a field $F$ and group $G$, we denote the group algebra of $G$ over $F$ by $F G$. Note that an $F$-vector space $V$ is a faithful $F G$-module if and only if $G \leqslant \mathrm{GL}(V)$. To emphasise that $V$ is a vector space over $F$, we write $\operatorname{GL}(V, F)$. An $F G$-module $V$ or a subgroup $G$ of $\operatorname{GL}(V, F)$ is 
irreducible if there are no proper $F G$-submodules of $V$. An irreducible subgroup $G$ of $\operatorname{GL}(V, F)$ is absolutely irreducible if $G$ is irreducible when viewed as a subgroup of $\operatorname{GL}(V, E)$ for all field extensions $E$ of $F$. An irreducible subgroup $G$ of $\operatorname{GL}(V, F)$ is absolutely irreducible if and only if $C_{\mathrm{GL}(V, F)}(G)=F^{*}$ by $[37,2.10 .1]$. We will use the following observation throughout this paper: if $G$ is an irreducible subgroup of $\mathrm{GL}(V)$ and $Z(G)$ contains an involution $z$, then $z=-1$.

Let $U$ and $W$ be vector spaces over the field $\mathbb{F}_{q}$, and let $V$ be the tensor product $U \otimes W$. For $g \in \mathrm{GL}(U)$ and $h \in \mathrm{GL}(W)$, let $(u \otimes w)^{g \otimes h}:=u^{g} \otimes w^{h}$ for all $u \in U$ and $w \in W$. Now $g \otimes h$ extends to a linear map of $V$. For $S \leqslant \mathrm{GL}(U)$ and $T \leqslant \mathrm{GL}(W)$, define $S \otimes T:=\{g \otimes h: g \in S, h \in T\}$. Then $S \otimes T \leqslant \mathrm{GL}(V)$ and $S \otimes T \simeq S \circ T$. There are natural actions of $\operatorname{Aut}\left(\mathbb{F}_{q}\right)$ on $V, U$ and $W$ for which $(u \otimes w)^{\sigma}=u^{\sigma} \otimes w^{\sigma}$ for all $u \in U, w \in W$ and $\sigma \in \operatorname{Aut}\left(\mathbb{F}_{q}\right)$, so that $(\mathrm{GL}(U) \otimes \mathrm{GL}(W)): \operatorname{Aut}\left(\mathbb{F}_{q}\right)$ stabilises the tensor decomposition of $V$.

2.4. Affine and projective planes and spaces. Recall from the introduction that a $2-(v, k, 1)$ design is a linear space with $v$ points and line-size $k$. For $n \geqslant 2$, a (finite) affine plane of order $n$ is a $2-\left(n^{2}, n, 1\right)$ design, and a (finite) projective plane of order $n$ is a $2-\left(n^{2}+n+1, n+1,1\right)$ design. Given a projective plane of order $n$, we obtain an affine plane of order $n$ by removing one line and all of its points. Conversely, given an affine plane $\mathcal{A}$ of order $n$, we obtain a projective plane of order $n$, called the completion of $\mathcal{A}$, by adding a point at infinity for every parallel class of lines, and defining the union of these new points to be the line at infinity, denoted by $\ell_{\infty}$.

For $m \geqslant 0$ and a prime power $q$, the affine space $\mathrm{AG}_{m}(q)$ is a linear space with points $V_{m}(q)$ and lines $\left\{\langle u\rangle+v: u \in V_{m}(q)^{*}, v \in V_{m}(q)\right\}$, while the projective space $\mathrm{PG}_{m}(q)$ or $\mathrm{PG}\left(V_{m+1}(q)\right)$ is a linear space whose points and lines are, respectively, the one- and two-dimensional subspaces of $V_{m+1}(q)$. An affine $\left(k\right.$-) subspace of $\mathrm{AG}_{m}(q)$ is a translation of a $k$-dimensional subspace of $V_{m}(q)$, and a projective $\left(k\right.$-) subspace of $\mathrm{PG}_{m}(q)$ is a $(k+1)$-dimensional subspace of $V_{m+1}(q)$; in particular, affine 2-subspaces are called planes, and projective $(m-1)$-subspaces are called hyperplanes. The affine space $\mathrm{AG}_{m}(q)$ may be obtained from $\mathrm{PG}_{m}(q)$ by removing a hyperplane $\mathcal{H}$ and its points and lines. With this viewpoint of $\mathrm{AG}_{m}(q)$, for $1 \leqslant k \leqslant m$, any affine $k$-subspace $W$ of $\mathrm{AG}_{m}(q)$ is the intersection of a projective $k$-subspace of $\mathrm{PG}_{m}(q)$ - the completion of $W$ with the complement of $\mathcal{H}$; further, the completion of $W$ meets $\mathcal{H}$ in a projective $(k-1)$-subspace. Note that $\mathrm{AG}_{2}(q)$ is the Desarguesian affine plane of order $q$, and $\mathrm{PG}_{2}(q)$ is the Desarguesian projective plane of order $q$.

2.5. Affine permutation groups. In the introduction, we defined an affine primitive permutation group to be a primitive group whose socle is a vector space over a field of prime order. In this section, for convenience, we expand this definition to include (certain) transitive groups.

First we require some notation. Let $q$ be a power of a prime $p$, and let $n$ be a positive integer. We denote the affine general linear group and affine semilinear group by $\operatorname{AGL}_{n}(q)$ and $\mathrm{A \Gamma L}_{n}(q)$, respectively. For $v \in V:=V_{n}(q)$, define $\tau_{v}: V \rightarrow V$ to be the translation $x \mapsto x+v$ for all $x \in V$. With some abuse of notation, we denote the group of translations of $V$ by $V$, so that $\operatorname{AGL}_{n}(q)=V: \operatorname{GL}_{n}(q)$ and $\operatorname{A\Gamma L}_{n}(q)=V: \Gamma L_{n}(q)$.

A group $G$ is an affine permutation group on $V:=V_{n}(q)$ whenever $V \leqslant G \leqslant \operatorname{A\Gamma L}_{n}(q)$. If $G$ is such a group, then $G=V: G_{0}$ and $G_{0} \leqslant \Gamma \mathrm{L}_{n}(q)$, where $G_{0}$ is the stabiliser of the zero vector. Note that if $q^{n}=p^{d}$, then $\Gamma \mathrm{L}_{n}(q) \leqslant \mathrm{GL}_{d}(p)$ and we may view $V$ as $V_{d}(p)$, so that $\mathrm{A \Gamma L}_{n}(q) \leqslant \mathrm{AGL}_{d}(p)$. The proof of the following is routine; see [22, Proposition 6.1.1].

Lemma 2.1. Let $G$ be an affine permutation group on $V:=V_{d}(p)$, where $d \geqslant 1$ and $p$ is prime. Then the following are equivalent.

(i) $G$ is primitive on $V$.

(ii) $V$ is an irreducible $\mathbb{F}_{p} G_{0}$-module.

Note that if an affine permutation group $G$ on $V$ is primitive, then $V$ is the socle of $G$ by [20, Theorem 4.3B]. Thus the definition from the introduction of an affine primitive permutation group coincides with the definition in this section of an affine permutation group that is primitive. 
If $G$ is an affine permutation group on $V$, then $G$ has rank 3 if and only if $G_{0}$ has two orbits on $V^{*}=V \backslash\{0\}$. This observation leads us to the following useful version of Lemma 2.1.

Lemma 2.2. Let $G$ be an affine permutation group of rank 3 on $V:=V_{d}(p)$, where $d \geqslant 1$ and $p$ is prime. Let $X$ and $Y$ be the orbits of $G_{0}$ on $V^{*}$. Then the following are equivalent.

(i) $G$ is primitive on $V$.

(ii) Neither $X \cup\{0\}$ nor $Y \cup\{0\}$ is an $\mathbb{F}_{p}$-subspace of $V$.

Proof. If $X \cup\{0\}$ is an $\mathbb{F}_{p}$-subspace of $V$, then $X \cup\{0\}$ is an $\mathbb{F}_{p} G_{0}$-submodule of $V$, so $G$ is imprimitive on $V$ by Lemma 2.1. Conversely, if $G$ is imprimitive on $V$, then $V$ has a proper non-zero $\mathbb{F}_{p} G_{0}$-submodule $W$ by Lemma 2.1 , but $W^{*}$ is preserved by $G_{0}$, so $W^{*}=X$ or $Y$.

Thus if $G$ and $H$ are affine permutation groups of rank 3 on $V:=V_{d}(p)$ where $G$ and $H$ have the same orbits on $V^{*}$, then $G$ is primitive on $V$ if and only if $H$ is primitive on $V$.

We conclude this section with an observation that we will use frequently to prove that some $\mathbb{F}_{p^{-}}$-subspace of $V$ is a line of a partial linear space (cf. Lemma 2.7).

Lemma 2.3. Let $G$ be an affine permutation group on $V:=V_{d}(p)$, where $d \geqslant 1$ and $p$ is prime. If $L$ is an $\mathbb{F}_{p}$-subspace of $V$, then $G_{L}$ is transitive on $L$.

Proof. Let $x \in L^{*}$. Then $0^{\tau_{x}}=x$ and $\tau_{x} \in G$, so it suffices to show that $L^{\tau_{x}} \subseteq L$. If $y \in L$, then $y^{\tau_{x}}=y+x \in L$, so $L^{\tau_{x}} \subseteq L$, as desired.

2.6. Partial linear spaces. Let $\mathcal{S}:=(\mathcal{P}, \mathcal{L})$ be a partial linear space. Distinct points $x, y \in \mathcal{P}$ are collinear if there exists a line $L \in \mathcal{L}$ containing $x$ and $y$; we also say that $x$ and $y$ lie on the line $L$, and so on. For $x \in \mathcal{P}$, let $\mathcal{L}_{x}$ denote the set of lines in $\mathcal{L}$ that contain the point $x$, and let $\mathcal{S}(x)$ denote the set of points in $\mathcal{P}$ that are collinear with $x$. The collinearity relation of $\mathcal{S}$ is the set of $(x, y) \in \mathcal{P} \times \mathcal{P}$ such that $x$ and $y$ are collinear, and the non-collinearity relation of $\mathcal{S}$ is the set of $(x, y) \in \mathcal{P} \times \mathcal{P}$ such that $x$ and $y$ are distinct and not collinear. The collinearity graph of $\mathcal{S}$ is the graph $(\mathcal{P},\{\{x, y\}:(x, y) \in \mathcal{R}\})$ where $\mathcal{R}$ is the collinearity relation of $\mathcal{S}$.

An isomorphism $\varphi: \mathcal{S} \rightarrow \mathcal{S}^{\prime}$ between $\mathcal{S}$ and a partial linear space $\mathcal{S}^{\prime}:=\left(\mathcal{P}^{\prime}, \mathcal{L}^{\prime}\right)$ is a bijection $\varphi: \mathcal{P} \rightarrow \mathcal{P}^{\prime}$ such that $\mathcal{L}^{\prime}=\mathcal{L}^{\varphi}$, where $\mathcal{L}^{\varphi}:=\left\{L^{\varphi}: L \in \mathcal{L}\right\}$ and $L^{\varphi}:=\left\{x^{\varphi}: x \in L\right\}$ for $L \in \mathcal{L}$. When such an isomorphism exists, we say that $\mathcal{S}$ and $\mathcal{S}^{\prime}$ are isomorphic. The automorphism group $\operatorname{Aut}(\mathcal{S})$ of $\mathcal{S}$ is $\left\{g \in \operatorname{Sym}(\mathcal{P}): \mathcal{L}^{g}=\mathcal{L}\right\}$. For $g \in \operatorname{Sym}(\mathcal{P})$, the pair $\left(\mathcal{P}, \mathcal{L}^{g}\right)$ is a partial linear space, which we denote by $\mathcal{S}^{g}$. Observe that $\mathcal{S}$ and $\mathcal{S}^{g}$ are isomorphic, and $\operatorname{Aut}\left(\mathcal{S}^{g}\right)=\operatorname{Aut}(\mathcal{S})^{g}$ for all $g \in \operatorname{Sym}(\mathcal{P})$.

A flag of $\mathcal{S}$ is a pair $(x, L)$ where $x$ is a point on a line $L$, and $\mathcal{S}$ is flag-transitive if $\operatorname{Aut}(\mathcal{S})$ acts transitively on the flags of $\mathcal{S}$. Similarly, $\mathcal{S}$ is point- or line-transitive if $\operatorname{Aut}(\mathcal{S})$ acts transitively on $\mathcal{P}$ or $\mathcal{L}$, respectively. Observe that if $\mathcal{S}$ is point- or line-transitive, then the point- or line-size of $\mathcal{S}$ is defined, respectively. Further, a flag-transitive partial linear space (with no isolated points) is point- and line-transitive. Lastly, if $\operatorname{Aut}(\mathcal{S})$ acts transitively on its collinearity relation, then $\mathcal{S}$ is flag-transitive.

We will use the following fundamental result throughout this paper; its proof is routine.

Lemma 2.4. Let $\mathcal{S}:=(\mathcal{P}, \mathcal{L})$ be a partial linear space with collinearity relation $\mathcal{R}_{1}$ and noncollinearity relation $\mathcal{R}_{2}$ where $\mathcal{R}_{1}$ and $\mathcal{R}_{2}$ are non-empty. Let $G \leqslant \operatorname{Aut}(\mathcal{S})$. Then the following are equivalent.

(i) $G$ is transitive of rank 3 on $\mathcal{P}$.

(ii) The orbits of $G$ on $\mathcal{P} \times \mathcal{P}$ are $\{(u, u): u \in \mathcal{P}\}, \mathcal{R}_{1}$ and $\mathcal{R}_{2}$.

(iii) $G$ is transitive on $\mathcal{P}$ and for $u \in \mathcal{P}$, the orbits of $G_{u}$ on $\mathcal{P}$ are $\{u\}, \mathcal{S}(u)=\{v \in \mathcal{P}$ : $\left.(u, v) \in \mathcal{R}_{1}\right\}$ and $\left\{v \in \mathcal{P}:(u, v) \in \mathcal{R}_{2}\right\}$.

Remark 2.5. If $\mathcal{S}$ is a proper partial linear space with collinearity relation $\mathcal{R}_{1}$ and noncollinearity relation $\mathcal{R}_{2}$, then $\mathcal{R}_{2}$ is non-empty (or else $\mathcal{S}$ is a linear space) and $\mathcal{R}_{1}$ is non-empty (or else $\mathcal{S}$ is a graph with no edges). In particular, for a proper partial linear space $\mathcal{S}:=(\mathcal{P}, \mathcal{L})$ 
with $G \leqslant \operatorname{Aut}(\mathcal{S})$ such that $G$ has rank 3 on $\mathcal{P}$, Lemma 2.4 implies that $\mathcal{S}$ is flag-transitive. Moreover, the orbitals of $G$ are self-paired, and $\operatorname{Aut}(\mathcal{S})$ has rank 3 on $\mathcal{P}$.

The following is a collection of necessary conditions for the existence of a partial linear space whose automorphism group is transitive on points and pairs of collinear points.

Lemma 2.6. Let $\mathcal{S}:=(\mathcal{P}, \mathcal{L})$ be a partial linear space with collinearity relation $\mathcal{R}$. Let $G \leqslant$ $\operatorname{Aut}(\mathcal{S})$ where $G$ is transitive on $\mathcal{P}$ and $\mathcal{R}$. Let $L \in \mathcal{L}$ and $u \in L$. Let $B:=L \backslash\{u\}$. Then the following hold.

(i) $B$ is a block of $G_{u}$ on $\mathcal{S}(u)$.

(ii) $G_{L}$ is 2-transitive on $L$.

(iii) If $\mathcal{S}$ is proper and $G$ is primitive on $\mathcal{P}$, then $B$ is a non-trivial block of $G_{u}$ on $\mathcal{S}(u)$.

Proof. (i) Suppose that $v \in B \cap B^{g}$ for some $g \in G_{u}$. Now $u$ and $v$ are distinct points on the lines $L$ and $L^{g}$, so $L=L^{g}$, in which case $B=B^{g}$.

(ii) Let $x, y, v, w \in L$ where $x \neq y$ and $v \neq w$. Now $(x, y),(v, w) \in \mathcal{R}$, so there exists $g \in G$ such that $x^{g}=v$ and $y^{g}=w$. In particular, $v, w \in L \cap L^{g}$, so $L=L^{g}$.

(iii) Suppose that $\mathcal{S}$ is proper. Then $|B| \geqslant 2$ and $|\mathcal{L}| \geqslant 2$. If $B=\mathcal{S}(u)$, then there is a unique line on $u$, so there is a unique line on every point of $\mathcal{S}$, but then $\mathcal{L}$ is a system of imprimitivity for $G$ on $\mathcal{P}$, so $G$ is imprimitive on $\mathcal{P}$.

Next we provide some sufficient conditions for the existence of a point-transitive partial linear space.

Lemma 2.7. Let $G$ be a transitive permutation group on $\mathcal{P}$. Let $u \in \mathcal{P}$, and let $B$ be a block of $G_{u}$ on $X$, where $X$ is an orbit of $G_{u}$ on $\mathcal{P} \backslash\{u\}$. Let $L:=B \cup\{u\}, \mathcal{L}:=L^{G}$ and $\mathcal{S}:=(\mathcal{P}, \mathcal{L})$, and suppose that $G_{L}$ is transitive on $L$. Then the following hold.

(i) $\mathcal{S}$ is a partial linear space with $G \leqslant \operatorname{Aut}(\mathcal{S})$ and $\mathcal{S}(u)=X$.

(ii) $\mathcal{S}$ is a linear space if and only if $G$ is 2-transitive on $\mathcal{P}$.

(iii) If $B$ is a non-trivial block, then $\mathcal{S}$ has line-size at least 3 and point-size at least 2.

(iv) If $B$ is a non-trivial block and $G$ has rank 3 on $\mathcal{P}$, then $\mathcal{S}$ is proper.

Proof. (i) The proof that $\mathcal{S}$ is a partial linear space is routine (see [16, Theorem 2.3] for details). Clearly $G \leqslant \operatorname{Aut}(\mathcal{S})$. If $v \in \mathcal{S}(u)$, then $v \in M$ for some $M \in \mathcal{L}_{u}$, and there exists $g \in G_{u}$ such that $M=L^{g}$ since $G_{L}$ is transitive on $L$, so $v \in B^{g} \subseteq X^{g}=X$. Conversely, if $v \in X$, then $v \in B^{g}$ for some $g \in G_{u}$, so $v \in \mathcal{S}(u)$.

(ii) $\mathcal{S}$ is a linear space if and only if $\mathcal{S}(u)=\mathcal{P} \backslash\{u\}$, so (ii) follows from (i).

(iii) Note that $\mathcal{S}$ is point- and line-transitive. If $B$ is non-trivial, then $|L| \geqslant 3$ and there are at least two lines on $u$, so (iii) holds.

(iv) This follows from (ii) and (iii).

In particular, if $G$ is a primitive permutation group of rank 3 on $\mathcal{P}$, then $\mathcal{S}:=(\mathcal{P}, \mathcal{L})$ is a proper partial linear space with $G \leqslant \operatorname{Aut}(\mathcal{S})$ if and only if $\mathcal{L}=L^{G}$ for some $L \subseteq \mathcal{P}$ such that $G_{L}$ is transitive on $L$ and $L \backslash\{u\}$ is a non-trivial block of $G_{u}$ on $v^{G_{u}}$ for some distinct $u, v \in L$.

The following result is standard and can be proved by counting flags.

Lemma 2.8. Let $\mathcal{S}:=(\mathcal{P}, \mathcal{L})$ be a partial linear space with line-size $k$ and point-size $\ell$. Then $|\mathcal{L}| k=|\mathcal{P}| \ell$ and $|\mathcal{S}(u)|=\ell(k-1)$ for all $u \in \mathcal{P}$.

In general, the automorphism group of a proper partial linear space can be primitive of rank 3 and have imprimitive subgroups. However, we now see that all rank 3 subgroups are primitive.

Lemma 2.9. Let $\mathcal{S}:=(\mathcal{P}, \mathcal{L})$ be a partial linear space with collinearity relation $\mathcal{R}_{1}$ and noncollinearity relation $\mathcal{R}_{2}$ where $\mathcal{R}_{1}$ and $\mathcal{R}_{2}$ are non-empty. Let $G \leqslant \operatorname{Aut}(\mathcal{S})$ where $G$ is transitive of rank 3 on $\mathcal{P}$. Then $G$ is primitive on $\mathcal{P}$ if and only if $\operatorname{Aut}(\mathcal{S})$ is primitive on $\mathcal{P}$. 
Proof. Suppose that $G$ is imprimitive on $\mathcal{P}$. Then there is a $G$-invariant equivalence relation $\mathcal{R}$ on $\mathcal{P}$ for which $\{(u, u): u \in \mathcal{P}\} \subsetneq \mathcal{R} \subsetneq \mathcal{P} \times \mathcal{P}$. By Lemma $2.4, \mathcal{R} \backslash\{(u, u): u \in \mathcal{P}\}$ is $\mathcal{R}_{1}$ or $\mathcal{R}_{2}$. Since $\mathcal{R}_{1}$ and $\mathcal{R}_{2}$ are $\operatorname{Aut}(\mathcal{S})$-invariant, $\operatorname{Aut}(\mathcal{S})$ is imprimitive. The converse is routine.

To finish this section, we make some brief observations about classifying partial linear spaces whose automorphism groups are imprimitive of rank 3 .

Remark 2.10. Let $\mathcal{S}:=(\mathcal{P}, \mathcal{L})$ be a partial linear space with collinearity relation $\mathcal{R}_{1}$ and non-collinearity relation $\mathcal{R}_{2}$ where $\mathcal{R}_{1}$ and $\mathcal{R}_{2}$ are non-empty. Let $\Gamma$ be the collinearity graph of $\mathcal{S}$. Then $\Gamma$ has the same collinearity and non-collinearity relations as $\mathcal{S}$. Let $G \leqslant \operatorname{Aut}(\mathcal{S})$ where $G$ is imprimitive of rank 3 on $\mathcal{P}$. By the proof of Lemma 2.9, $\mathcal{R}_{i} \cup\{(u, u): u \in \mathcal{P}\}$ is an equivalence relation with $m$ classes of size $n$ for some $i \in\{1,2\}$ and $m, n \geqslant 2$. If $i=1$, then $\Gamma$ is a disjoint union of $m$ complete graphs of size $n$, so $\mathcal{S}$ is a disjoint union of isomorphic 2 -transitive linear spaces (and such linear spaces were classified in [36]). Otherwise, $i=2$ and $\Gamma$ is a complete multipartite graph $K_{m[n]}$ with $m$ parts of size $n$. Thus, in order to classify the proper partial linear spaces admitting rank 3 imprimitive automorphism groups, it suffices to consider those with collinearity graph $K_{m[n]}$. This difficult problem has been studied in certain cases (see $[18,48]$ ) but remains open in general.

2.7. Constructing new partial linear spaces from given ones. Let $\mathcal{S}:=(\mathcal{P}, \mathcal{L})$ be a linear space with at least three points on every line, let $G \leqslant \operatorname{Aut}(\mathcal{S})$ such that $G$ has rank 3 on $\mathcal{P}$, and let $u \in \mathcal{P}$. Now $G_{u}$ has two orbits $X$ and $Y$ on $\mathcal{P} \backslash\{u\}$. If there exists $L \in \mathcal{L}_{u}$ such that $X \cap L$ and $Y \cap L$ are non-empty, then it is not hard to see that $\mathcal{S}$ is flag-transitive. Otherwise, $G$ has exactly two orbits on $\mathcal{L}$, and, as we discussed in Remark 1.5, we can use these orbits to construct partial linear spaces that admit $G$. We consider this construction in more detail now.

Lemma 2.11. Let $G$ be a rank 3 permutation group on $\mathcal{P}$. Then the following are equivalent.

(i) $\mathcal{S}:=\left(\mathcal{P}, \mathcal{L}_{1} \cup \mathcal{L}_{2}\right)$ is a linear space with at least three points on every line such that $G \leqslant \operatorname{Aut}(\mathcal{S})$ and $G$ has distinct orbits $\mathcal{L}_{1}$ and $\mathcal{L}_{2}$ on $\mathcal{L}_{1} \cup \mathcal{L}_{2}$.

(ii) $\mathcal{S}_{1}:=\left(\mathcal{P}, \mathcal{L}_{1}\right)$ and $\mathcal{S}_{2}:=\left(\mathcal{P}, \mathcal{L}_{2}\right)$ are proper partial linear spaces with $G \leqslant \operatorname{Aut}\left(\mathcal{S}_{1}\right)$ and $G \leqslant \operatorname{Aut}\left(\mathcal{S}_{2}\right)$ such that the collinearity relations of $\mathcal{S}_{1}$ and $\mathcal{S}_{2}$ are disjoint.

Proof. First suppose that (i) holds. Clearly $\mathcal{S}_{1}$ and $\mathcal{S}_{2}$ are proper partial linear spaces such that $G \leqslant \operatorname{Aut}\left(\mathcal{S}_{1}\right)$ and $G \leqslant \operatorname{Aut}\left(\mathcal{S}_{2}\right)$. If there exist $u, v \in \mathcal{P}$ such that $u$ and $v$ are collinear in both $\mathcal{S}_{1}$ and $\mathcal{S}_{2}$, then there exist $L_{1} \in \mathcal{L}_{1}$ and $L_{2} \in \mathcal{L}_{2}$ such that $u, v \in L_{1} \cap L_{2}$, but then $L_{1}=L_{2}$, so $\mathcal{L}_{1}$ and $\mathcal{L}_{2}$ are not disjoint, a contradiction. Thus (ii) holds.

Conversely, suppose that (ii) holds, and let $\mathcal{R}_{i}$ be the collinearity relation of $\mathcal{S}_{i}$ for $i \in\{1,2\}$. Since $\mathcal{R}_{1}$ and $\mathcal{R}_{2}$ are disjoint, $\mathcal{L}_{1}$ and $\mathcal{L}_{2}$ are disjoint, and $\mathcal{S}$ is a partial linear space. Observe that $\mathcal{L}_{1}$ and $\mathcal{L}_{2}$ are both non-empty since $\mathcal{S}_{1}$ and $\mathcal{S}_{2}$ are not graphs. Now $\mathcal{R}_{1}$ and $\mathcal{R}_{2}$ are both non-empty, so the orbits of $G$ on $\mathcal{P} \times \mathcal{P}$ are $\{(u, u): u \in \mathcal{P}\}, \mathcal{R}_{1}$ and $\mathcal{R}_{2}$. If $u$ and $v$ are distinct elements of $\mathcal{P}$, then $(u, v) \in \mathcal{R}_{i}$ for some $i$, so there exists $L \in \mathcal{L}_{i}$ such that $u, v \in L$. Thus $\mathcal{S}$ is a linear space. Clearly $G \leqslant \operatorname{Aut}(\mathcal{S})$, and $G$ is transitive on $\mathcal{L}_{1}$ and $\mathcal{L}_{2}$, so (i) holds.

Note that the partial linear spaces $\mathcal{S}_{1}$ and $\mathcal{S}_{2}$ of Lemma 2.11 have the same line-size if and only if $\mathcal{S}$ is a $2-(v, k, 1)$ design for some $v$ and $k$.

Remark 2.12. Observe that if $\mathcal{S}:=(\mathcal{P}, \mathcal{L})$ and $\mathcal{S}^{\prime}:=\left(\mathcal{P}^{\prime}, \mathcal{L}^{\prime}\right)$ are isomorphic linear spaces that satisfy the conditions of Lemma 2.11(i), then any isomorphism $\varphi: \mathcal{S} \rightarrow \mathcal{S}^{\prime}$ naturally determines isomorphisms between the corresponding partial linear spaces: indeed, if $\mathcal{L}_{1}$ and $\mathcal{L}_{2}$ are the orbits of the rank 3 group $G$ on $\mathcal{L}$, then $\mathcal{L}_{1}^{\prime}:=\mathcal{L}_{1}^{\varphi}$ and $\mathcal{L}_{2}^{\prime}:=\mathcal{L}_{2}^{\varphi}$ are the orbits of $G^{\varphi}$ on $\mathcal{L}^{\prime}$, so $\varphi$ is an isomorphism between the partial linear spaces $\left(\mathcal{P}, \mathcal{L}_{1}\right)$ and $\left(\mathcal{P}^{\prime}, \mathcal{L}_{1}^{\prime}\right)$, as well as $\left(\mathcal{P}, \mathcal{L}_{2}\right)$ and $\left(\mathcal{P}^{\prime}, \mathcal{L}_{2}^{\prime}\right)$. However, the converse is not true in general: see Remark 11.5.

Next we define the intersection of two partial linear spaces. Let $\mathcal{S}_{1}:=\left(\mathcal{P}_{1}, \mathcal{L}_{1}\right)$ and $\mathcal{S}_{2}:=$ $\left(\mathcal{P}_{2}, \mathcal{L}_{2}\right)$ be partial linear spaces. Define $\mathcal{S}_{1} \cap \mathcal{S}_{2}$ to be the pair $\left(\mathcal{P}_{1} \cap \mathcal{P}_{2}, \mathcal{L}\right)$ where $\mathcal{L}:=\left\{L_{1} \cap L_{2}\right.$ : 
$\left.L_{1} \in \mathcal{L}_{1}, L_{2} \in \mathcal{L}_{2},\left|L_{1} \cap L_{2}\right| \geqslant 2\right\}$. Further, if $\mathcal{P} \subseteq \mathcal{P}_{1}$ such that $|\mathcal{P}|>1$, then $\mathcal{S}:=(\mathcal{P},\{\mathcal{P}\})$ is a linear space, and we write $\mathcal{S}_{1} \cap \mathcal{P}$ for $\mathcal{S}_{1} \cap \mathcal{S}$. The proof of the following is routine.

Lemma 2.13. Let $\mathcal{S}_{1}:=\left(\mathcal{P}_{1}, \mathcal{L}_{1}\right)$ and $\mathcal{S}_{2}:=\left(\mathcal{P}_{2}, \mathcal{L}_{2}\right)$ be partial linear spaces.

(i) If $\mathcal{P}_{1} \cap \mathcal{P}_{2} \neq \varnothing$, then $\mathcal{S}_{1} \cap \mathcal{S}_{2}$ is a partial linear space.

(ii) If $\mathcal{P} \subseteq \mathcal{P}_{1}$ such that $|\mathcal{P}|>1$ and $G \leqslant \operatorname{Aut}\left(\mathcal{S}_{1}\right)$, then $G_{\mathcal{P}}^{\mathcal{P}} \leqslant \operatorname{Aut}\left(\mathcal{S}_{1} \cap \mathcal{P}\right)$.

Our final construction of this section is a natural generalisation of the cartesian product of graphs. Let $\mathcal{S}_{1}:=\left(\mathcal{P}_{1}, \mathcal{L}_{1}\right)$ and $\mathcal{S}_{2}:=\left(\mathcal{P}_{2}, \mathcal{L}_{2}\right)$ be partial linear spaces. Define the cartesian product $\mathcal{S}_{1} \square \mathcal{S}_{2}$ of $\mathcal{S}_{1}$ and $\mathcal{S}_{2}$ to be $\left(\mathcal{P}_{1} \times \mathcal{P}_{2}, \mathcal{L}_{1} \square \mathcal{L}_{2}\right)$ where $\mathcal{L}_{1} \square \mathcal{L}_{2}$ is the union of $\left\{\left\{\left(x_{1}, x_{2}\right)\right.\right.$ : $\left.\left.x_{1} \in L\right\}: x_{2} \in \mathcal{P}_{2}, L \in \mathcal{L}_{1}\right\}$ and $\left\{\left\{\left(x_{1}, x_{2}\right): x_{2} \in L\right\}: x_{1} \in \mathcal{P}_{1}, L \in \mathcal{L}_{2}\right\}$.

Lemma 2.14. Let $\mathcal{S}_{1}:=\left(\mathcal{P}_{1}, \mathcal{L}_{1}\right)$ and $\mathcal{S}_{2}:=\left(\mathcal{P}_{2}, \mathcal{L}_{2}\right)$ be partial linear spaces.

(i) $\mathcal{S}_{1} \square \mathcal{S}_{2}$ is a partial linear space, and $\operatorname{Aut}\left(\mathcal{S}_{1}\right) \times \operatorname{Aut}\left(\mathcal{S}_{2}\right) \leqslant \operatorname{Aut}\left(\mathcal{S}_{1} \square \mathcal{S}_{2}\right)$.

(ii) $\operatorname{Aut}\left(\mathcal{S}_{1}\right) 2\langle\tau\rangle \leqslant \operatorname{Aut}\left(\mathcal{S}_{1} \square \mathcal{S}_{1}\right)$ where $\left(x_{1}, x_{2}\right)^{\tau}:=\left(x_{2}, x_{1}\right)$ for all $x_{1}, x_{2} \in \mathcal{P}_{1}$.

(iii) If $\left|\mathcal{P}_{1}\right|>1$, then $\mathcal{S}_{1} \square \mathcal{S}_{1}$ is not a linear space.

Proof. Suppose that there exist distinct $\left(x_{1}, x_{2}\right),\left(y_{1}, y_{2}\right) \in L \cap M$ for some $L, M \in \mathcal{L}_{1} \square \mathcal{L}_{2}$. We may assume that $x_{1} \neq y_{1}$. Then $x_{2}=y_{2}$, so $L=\left\{\left(u, x_{2}\right): u \in L^{\prime}\right\}$ and $M=\left\{\left(v, x_{2}\right): v \in M^{\prime}\right\}$ for some $L^{\prime}, M^{\prime} \in \mathcal{L}_{1}$ such that $x_{1}, y_{1} \in L^{\prime} \cap M^{\prime}$. Now $L^{\prime}=M^{\prime}$, so $L=M$. Thus $\mathcal{S}_{1} \square \mathcal{S}_{2}$ is a partial linear space. The remaining claims of (i) and (ii) are straightforward. If there exist distinct $x_{1}, x_{2} \in \mathcal{P}_{1}$, then $\left(x_{1}, x_{2}\right)$ and $\left(x_{2}, x_{1}\right)$ are not collinear in $\mathcal{S}_{1} \square \mathcal{S}_{1}$, so (iii) holds.

2.8. Affine partial linear spaces. A partial linear space $S:=(\mathcal{P}, \mathcal{L})$ is $G$-affine if $G \leqslant \operatorname{Aut}(\mathcal{S})$ and $G$ is an affine permutation group on $V:=V_{d}(p)=\mathcal{P}$ (see $\S 2.5$ ) for some prime $p$ and $d \geqslant 1$. We also say that $\mathcal{S}$ is affine if it is $G$-affine for some $G$. Recall that for $x \in V$, we write $\mathcal{L}_{x}$ for the set of lines of $\mathcal{S}$ that contain $x$, and $\mathcal{S}(x)$ for the set of points of $\mathcal{S}$ that are collinear with $x$. In particular, $\mathcal{L}_{0}$ denotes the set of lines on the vector 0 , and $\mathcal{S}(0)$ denotes the set of vectors that are collinear with 0 . Recall also that if $L \subseteq V$, then $L^{*}:=L \backslash\{0\}$.

The first result of this section consists of two elementary observations that are the key tools for determining - or rather limiting - the structure of a $G$-affine proper partial linear space. In particular, there is a useful tension between Lemmas 2.15(i) and 2.2.

Lemma 2.15. Let $V:=V_{d}(p)$ where $d \geqslant 1$ and $p$ is prime, and let $\mathcal{S}:=(V, \mathcal{L})$ be a $G$-affine proper partial linear space where $G$ has rank 3 on $V$. Let $L \in \mathcal{L}_{0}$.

(i) If $x, y \in L^{*}$ and $x \neq y$, then $y-x \in x^{G_{0}}$.

(ii) If $x, y \in L^{*}$ and $x \neq y$, then $y^{G_{0, x}} \subseteq L^{*}$.

Proof. (i) By assumption, $V \leqslant G$, and $x$ and $y$ are collinear, so $x^{\tau_{-x}}$ and $y^{\tau_{-x}}$ are also collinear. Thus $y-x \in \mathcal{S}(0)$, and $\mathcal{S}(0)=x^{G_{0}}$ by Lemma 2.4 since $x \in \mathcal{S}(0)$ and $G$ has rank 3 on $V$.

(ii) If $z=y^{g}$ for some $g \in G_{0, x}$, then $L^{g}=L$, so $z \in\left(L^{*}\right)^{g}=L^{*}$.

A subset $L$ of a vector space $V$ is an affine subspace of $V$ if $L=W+v$ for some subspace $W$ of $V$ and $v \in V$. In the following, we generalise [40, Lemma 2.3] and the proof of [40, Lemma 2.2].

Lemma 2.16. Let $V:=V_{d}(p)$ where $d \geqslant 1$ and $p$ is prime. Let $\mathcal{S}:=(V, \mathcal{L})$ be a $G$-affine partial linear space where $G$ is transitive on $\mathcal{L}$ and $\mathcal{S}$ has line-size at least 3 . Suppose that one of the following holds.

(i) There exists $g \in G_{0}$ and $L \in \mathcal{L}_{0}$ such that $v^{g}=-v$ for all $v \in L$.

(ii) The prime $p=2$.

(iii) There exists $x \in V^{*}$ and $L \in \mathcal{L}$ such that $L^{\tau_{x}}=L$.

Then every line of $\mathcal{S}$ is an affine subspace of $V$.

Proof. By assumption, $V \leqslant G$. Suppose that (i) holds. Let $y, z \in L^{*}$ be distinct. Applying $g$ to $0, y$ and $z$, we see that $0,-y$ and $-z$ lie on a line, and we may translate by $y$, so $y, 0$ and $y-z$ lie on a line. Now $L$ is the unique line on 0 and $y$, so $y-z \in L^{*}$. Similarly, $z-y \in L^{*}$. Since 
$z-y \neq z$, we also have $-y=(z-y)-z \in L^{*}$. It follows that $u-v \in L$ for all $u, v \in L$, and

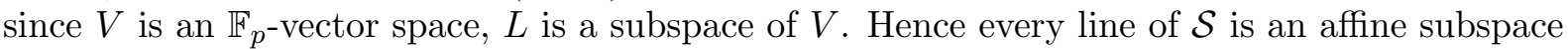
of $V$, for if $M \in \mathcal{L}$, then $M=L^{h}+v$ for some $h \in G_{0}$ and $v \in V$.

If the condition of (ii) holds, then the condition of (i) is satisfied with $g=1$ for any line $L$, so every line is an affine subspace of $V$.

Suppose that the condition of (iii) holds. First we claim that $L^{\tau_{v-u}}=L$ for all $u, v \in L$. Let $u, v \in L$. Now $u+\langle x\rangle$ and $v+\langle x\rangle$ are subsets of $L$, and $(u+\langle x\rangle)^{\tau_{v-u}}=v+\langle x\rangle$. Since $x \neq 0$, it follows that $\left|L^{\tau_{v-u}} \cap L\right| \geqslant 2$, so $L^{\tau_{v-u}}=L$, and the claim holds. Choose $y \in L$, and let $W:=\{u-y: u \in L\}$. Now $L=W+y$, and $W$ is a subspace of $V$ by the claim, so $L$ is an affine subspace of $V$. It follows that every line of $\mathcal{S}$ is an affine subspace of $V$.

Lemma 2.17. Let $V:=V_{d}(p)$ where $d \geqslant 1$ and $p$ is prime, and let $\mathcal{S}:=(V, \mathcal{L})$ be a $G$-affine proper partial linear space where $G$ has rank 3 on $V$. Then one of the following holds.

(i) Every line of $\mathcal{S}$ is an affine subspace of $V$.

(ii) The translation group $V$ acts semiregularly on $\mathcal{L}$ and $k(k-1)$ divides $\left|x^{G_{0}}\right|$, where $k$ is the line-size of $\mathcal{S}$ and $x \in \mathcal{S}(0)$.

Proof. Suppose that (i) does not hold. Now the translation group $V$ acts semiregularly on $\mathcal{L}$ by Lemma 2.16. Hence $|V|$ divides $|\mathcal{L}|$, so (ii) holds by Lemma 2.8 since $\mathcal{S}(0)=x^{G_{0}}$.

2.9. Nearfield planes. In Remark 1.5, we described how some of the partial linear spaces in Table 1 can be obtained from affine planes using rank 3 groups. In this section, we construct a particular family of these affine planes: the nearfield planes.

A nearfield $(Q,+, \circ)$ is a set $Q$ with binary operations + and $\circ$ such that the following four axioms hold.

(N1) $(Q,+)$ is an abelian group.

(N2) $(a+b) \circ c=a \circ c+b \circ c$ for all $a, b, c \in Q$.

(N3) $(Q \backslash\{0\}, \circ)$ is a group.

(N4) $a \circ 0=0$ for all $a \in Q$.

By (N2), $0 \circ b=0$ for all $b \in Q$. It then follows from (N2) that $(-a) \circ b=-(a \circ b)$ for all $a, b \in Q$.

A nearfield plane is constructed using a nearfield $(Q,+, \circ)$ as follows. For $w \in Q$, let $L(w):=$ $\{(v, v \circ w): v \in Q\}$, and let $L(\infty):=\{(0, v): v \in Q\}$. The nearfield plane on $Q$ has point set $Q \times Q$ and line set $\{L(w)+x: w \in Q \cup\{\infty\}, x \in Q \times Q\}$. Note that $(L(w),+) \simeq(Q,+)$ for $w \in Q \cup\{\infty\}$. Further, for each $(a, b) \in(Q \times Q) \backslash\{(0,0)\}$, there is a unique $w \in Q \cup\{\infty\}$ such that $(a, b) \in L(w)$ by (N3). Thus the nearfield plane on $Q$ is an affine plane of order $|Q|$.

Next we describe how to construct a nearfield using a sharply 2-transitive group. Let $V:=$ $V_{n}(q)$ where $n \geqslant 1$ and $q$ is a power of a prime $p$. Let $R$ be a subgroup of $\Gamma L_{n}(q)$ that is regular on $V^{*}=V \backslash\{0\}$, so $V: R$ acts sharply 2-transitively on $V$. Let $x \in V^{*}$. For $w \in V^{*}$, let $r_{w}$ denote the unique element of $R$ for which $w=x^{r_{w}}$, and for $v \in V$, let $v \circ w:=v^{r_{w}}$ and $v \circ 0:=0$. If $a, b \in V$ and $c \in V^{*}$, then $a \circ c+b \circ c=a^{r_{c}}+b^{r_{c}}=(a+b)^{r_{c}}=(a+b) \circ c$. Further, $r_{v} r_{w}=r_{v \circ w}$ for all $v, w \in V^{*}$, so $\left(V^{*}, \circ\right)$ is a group (and isomorphic to $R$ ). Thus $(V,+, \circ)$ is a nearfield.

The possibilities for $R$ are given by Zassenhaus's classification [57] of the sharply 2-transitive groups (see [20, §7.6]): either $n=1$, or $n=2$ and $q=p \in\{5,7,11,23,29,59\}$, in which case there are seven possibilities for $R$ (two of which occur for $p=11$ ), and the corresponding nearfield plane is an irregular nearfield plane of order $p^{2}$. See $[43, \S 7]$ for more details. Note that $\mathrm{AG}_{2}(q)$ is the nearfield plane that arises by taking $R=\mathrm{GL}_{1}(q)$. The (non-Desarguesian) nearfield plane of order 9 arises by taking $R=Q_{8} \leqslant \Gamma L_{1}(9)$; see $[43, \S 8]$ for more details.

2.10. Affine 2-transitive groups and linear spaces. The classification of the 2-transitive affine permutation groups was obtained by Huppert [30] in the soluble case (see also [24]), and by Hering [28] in general. Liebeck provides another proof of Hering's result in [39, Appendix 1]. The statement we give is similar to [39], except that we add a description of the affine 2-transitive subgroups of $\mathrm{A \Gamma L}_{2}(q)$ and $\mathrm{A \Gamma L}_{3}(q)$ for the proof of Theorem 3.2. 
Theorem 2.18 ([28, 39]). Let $G$ be a 2-transitive affine permutation group with socle $V:=V_{d}(p)$ where $d \geqslant 1$ and $p$ is prime. Then the stabiliser $G_{0}$ belongs to one of the following classes.

(H0) $G_{0} \leqslant \Gamma L_{1}(q)$ where $q=p^{d}$.

(H1) $\mathrm{SL}_{n}(q) \unlhd G_{0}$ where $q^{n}=p^{d}, n \geqslant 2$ and $(n, q) \neq(2,2)$ or $(2,3)$.

(H2) $\operatorname{Sp}_{2 n}(q) \unlhd G_{0}$ where $q^{2 n}=p^{d}$ and $n \geqslant 2$.

(H3) $G_{2}(q)^{\prime} \unlhd G_{0}$ where $q^{6}=p^{d}$ and $q$ is even.

(H4) $\mathrm{SL}_{2}(5) \unlhd G_{0} \leqslant \Gamma L_{2}(q)$ where $q^{2}=p^{d}$ and $q \in\{9,11,19,29,59\}$.

(H5) $G_{0}=A_{6}$ or $A_{7}$ and $p^{d}=2^{4}$.

(H6) $G_{0}=\mathrm{SL}_{2}(13)$ and $p^{d}=3^{6}$.

(H7) $\mathrm{SL}_{2}(3) \unlhd G_{0}$ where $d=2$ and $p \in\{3,5,7,11,23\}$. Here $\mathrm{SL}_{2}(3)$ is irreducible on $V$.

(H8) $D_{8} \circ Q_{8} \unlhd G_{0}$ where $p^{d}=3^{4}$. Here $D_{8} \circ Q_{8}$ is irreducible on $V$.

Further, if $G_{0} \leqslant \Gamma \mathrm{L}_{m}(r)$ where $r^{m}=p^{d}$ and $m=2$ or 3 , then one of the following holds: $G_{0}$ belongs to (H0); $G_{0}$ belongs to (H1) with $n=m$; or $m=2$ and $G_{0}$ belongs to (H4) or (H7).

Proof. Let $a$ be the minimal divisor of $d$ for which $G_{0} \leqslant \Gamma \mathrm{L}_{a}\left(p^{d / a}\right)$. Let $s:=p^{d / a}$. If $a=1$, then (H0) holds, so we assume that $a \geqslant 2$. In particular, $(a, s) \neq(2,2)$ since $\mathrm{GL}_{2}(2)=\Gamma L_{1}(4)$. By $[39$, Appendix 1], one of the following holds: (i) $\mathrm{SL}_{a}(s) \unlhd G_{0}$; (ii) $\operatorname{Sp}_{a}(s) \unlhd G_{0}$; (iii) $G_{2}(s)^{\prime} \unlhd G_{0}$ and $(a, p)=(6,2)$; (iv) $\mathrm{SL}_{2}(5) \unlhd G_{0}, a=2$ and $s \in\{9,11,19,29,59\}$; (v) $G_{0}=A_{6}$ or $A_{7}$ and $(a, s)=(4,2)$; (vi) $G_{0}=\mathrm{SL}_{2}(13)$ and $(a, s)=(6,3)$; (vii) $G_{0}$ normalises $E$ where $E \leqslant \mathrm{GL}_{d}(p)$ and either $E=Q_{8}$ and $p^{d} \in\left\{5^{2}, 7^{2}, 11^{2}, 23^{2}\right\}$, or $E=D_{8} \circ Q_{8}$ and $p^{d}=3^{4}$. If (i) holds, then either (H1) holds with $n=a$, or (H7) holds with $(a, s)=(2,3)$. If (ii) holds, then (H2) holds with $a=2 n$ where $n \geqslant 2$ since $\operatorname{Sp}_{2}(q)=\mathrm{SL}_{2}(q)$. If one of (iii), (iv), (v) or (vi) holds, then (H3), (H4), (H5) or (H6) holds, respectively. If (vii) holds with $E=Q_{8}$, then $a=2$, and (H7) holds by a computation in Magma. Lastly, if (vii) holds and $E=D_{8} \circ Q_{8}$, then by a computation in MAGMA, either $a=2$ and (H4) holds, or $a=4$ and (H8) holds (cf. Remark 2.20).

Suppose that $G_{0} \leqslant \Gamma L_{m}(r)$ where $r^{m}=p^{d}$ and $m=2$ or 3 . Then $a \leqslant m$. If $a=1$ or $m$, then we are done by the observations above. Otherwise, $a=2$ and $m=3$. Now $s^{2}=r^{3}$, so $s=t^{3}$ and $r=t^{2}$ where $t^{6}=p^{d}$. Further, (H1) holds and $S:=\mathrm{SL}_{2}\left(t^{3}\right) \unlhd G_{0}$. Since $S$ is perfect, $S \leqslant \mathrm{GL}_{3}\left(t^{2}\right)$, so $C:=C_{\mathrm{GL}_{6}(t)}(S)$ contains both $Z:=Z\left(\mathrm{GL}_{2}\left(t^{3}\right)\right)$ and $Z\left(\mathrm{GL}_{3}\left(t^{2}\right)\right)$. However, $S$ is irreducible on $V=V_{6}(t)$, so by Schur's lemma (see [32, Lemma 1.5]), $k:=\operatorname{Hom}_{\mathbb{F}_{t} S}(V, V)=C_{\operatorname{End}(V)}(S)$ is a division ring. Thus $k$ is a field by Wedderburn's theorem. Since $V$ is naturally a faithful $k S$-module, $t^{6}=|V|=|k|^{i}$ where $i \geqslant 2$, but $\mathbb{F}_{t^{3}} \subseteq k$, so $k=\mathbb{F}_{t^{3}}$ and $C=Z$, a contradiction.

Throughout this paper, we say that an affine 2-transitive permutation group $G$ or its stabiliser $G_{0}$ belongs to (or lies in, is contained in, etc.) one of the classes (H0)-(H8) whenever $G_{0}$ satisfies the given description. Note that the groups in class (H7) are all soluble.

Next we state Kantor's classification [36] of the 2-transitive affine linear spaces. Four exceptional linear spaces arise: the nearfield plane of order 9 (see $\S 2.9$ or $[43, \S 8]$ ), the Hering plane of order 27 (see [15, p.236]) and two Hering spaces on $3^{6}$ points with line-size 9 [29].

Theorem 2.19 ([36]). Let $G$ be a 2-transitive affine permutation group with socle $V:=V_{d}(p)$ where $d \geqslant 1$ and $p$ is prime. Then $\mathcal{S}$ is a $G$-affine linear space with line-size at least 3 if and only if one of the following holds.

(i) $\mathcal{S}$ has line set $\{V\}$ and $p^{d} \neq 2$.

(ii) $G_{0} \leqslant \Gamma L_{m}(q)$ where $q^{m}=p^{d}$ and $\mathcal{S}$ has line set $\left\{\langle u\rangle_{F}+v: u \in V^{*}, v \in V\right\}$, where $F$ is a subfield of $\mathbb{F}_{q}$ and $|F| \neq 2$.

(iii) $G_{0} \leqslant N_{\mathrm{GL}_{4}(3)}\left(D_{8} \circ Q_{8}\right)$ where $p^{d}=3^{4}$ and one of the following holds, where $\mathcal{N}$ is the nearfield plane of order 9 .

(a) $D_{8} \circ Q_{8} \unlhd G_{0}$ and $\mathcal{S}=\mathcal{N}$.

(b) $G_{0}=\mathrm{SL}_{2}(5)\left\langle\zeta_{9} \sigma_{9}\right\rangle \leqslant \Gamma L_{2}(9)$ and $\mathcal{S}=\mathcal{N}$ or $\mathcal{N}^{z}$ where $z:=\zeta_{9}^{2}$.

(iv) $G_{0}=\mathrm{SL}_{2}(13)$ where $p^{d}=3^{6}$ and $\mathcal{S}$ is the Hering plane of order 27 or either of the two Hering spaces with line-size 9. 
Moreover, if $G_{0}$ lies in (H0)-(H3), then (i) or (ii) holds, and if $G_{0}$ lies in (H5), then (i) holds.

Proof. This follows from [36, §4] and, in cases (iii) and (iv), a computation in MAGMA using Lemmas 2.6 and 2.16.

Remark 2.20. The automorphism group of the nearfield plane of order 9 is $V_{4}(3)$ : $N_{0}$ where $N_{0}:=N_{\mathrm{GL}_{4}(3)}\left(D_{8} \circ Q_{8}\right)=\left(D_{8} \circ Q_{8}\right) . S_{5}=D_{8} \circ Q_{8} \circ\left(\mathrm{SL}_{2}(5)\left\langle\zeta_{9} \sigma_{9}\right\rangle\right)$ with $\mathrm{SL}_{2}(5) \leqslant \mathrm{SL}_{2}(9)$. Further, if $G_{0} \leqslant N_{0}$ such that $G_{0}$ is transitive on $V_{4}(3)^{*}$, then either $D_{8} \circ Q_{8} \unlhd G_{0}$, or $G_{0}=\mathrm{SL}_{2}(5)\left\langle\zeta_{9} \sigma_{9}\right\rangle$. Note that $\mathrm{SL}_{2}(5)\left\langle\zeta_{9} \sigma_{9}\right\rangle \simeq 2 \cdot S_{5}^{-}$(see $\S 2.2$ for the definition of $2 \cdot S_{5}^{-}$).

2.11. Proof of Theorem 1.8. First we state the well-known structure theorem for primitive permutation groups of rank 3, which follows from the O'Nan-Scott Theorem (see [39, 41]). The groups in (ii) are said to be of grid type.

Proposition 2.21. For a primitive permutation group $G$ of rank 3 with degree $n$, one of the following holds.

(i) $G$ is almost simple.

(ii) $T \times T \unlhd G \leqslant K \backslash S_{2}$ and $n=m^{2}$, where $K$ is a 2-transitive group of degree $m$ that is almost simple with socle $T$, and $G$ has subdegrees $1,2(m-1)$ and $(m-1)^{2}$.

(iii) $G$ is affine.

We will use Proposition 2.21 to determine the automorphism groups of certain affine proper partial linear spaces, together with the following result.

Theorem 2.22 ([27]). Let $T$ be a non-abelian simple group that is transitive of degree $p^{d}$ for some prime $p$ and positive integer $d$. Then either $T$ is 2 -transitive, or $T \simeq \operatorname{PSU}_{4}(2)$ and $p^{d}=27$, in which case $T$ is primitive of rank 3 with subdegrees 1,10 and 16.

Any non-trivial normal subgroup of a primitive group is transitive, so if $G$ is an almost simple primitive group whose degree is a prime power not equal to 27 , then $G$ is 2 -transitive by Theorem 2.22.

Proof of Theorem 1.8. Let $H:=\operatorname{Aut}(\mathcal{S})$. Since $\mathcal{S}$ is proper and $G$ is a primitive permutation group of rank 3 on $V$, Remark 2.5 implies that $H$ is a primitive permutation group of rank 3 on $V$. Note that $H$ has degree $p^{d}$. If $H$ is almost simple with socle $T$, then $T$ is transitive of degree $p^{d}$. Since $H$ is not 2-transitive, $T \simeq \operatorname{PSU}_{4}(2)$ and $p^{d}=27$ by Theorem 2.22 , but then $H$ has no affine primitive subgroups by a computation in MAgma. (This also follows from [49, Proposition 5.1], except that the one possibility described for $G$ in [49, Table 2] is not primitive as claimed, and this is corrected in [50, Proposition 2.1].) If $H$ is affine, then by [49, Proposition 5.1], the socle of $G$ is equal to the socle of $H$, so (ii) holds. Otherwise, by Proposition 2.21, $T \times T \unlhd H \leqslant K \imath S_{2}$ and $d=2 n$, where $K$ is a 2-transitive group of degree $p^{n}$ that is almost simple with socle $T$. If $T=A_{p^{n}}$, then it is routine to verify that (i) holds (see [17] for details). If $T=\operatorname{PSU}_{4}(2)$ and $p^{n}=27$, then the order of $K$ is not divisible by $p^{n}-1$, so $K$ is not 2-transitive, a contradiction. Thus, by [49, Proposition 5.1], $K$ has degree $p$ and is listed in [49, Table 2].

Since $G$ is an affine primitive subgroup of $K$ ? $S_{2}$ with rank 3, the subgroup $G \cap(K \times K)$ has index 2 in $G$, and the projection $G^{1}$ of $G \cap(K \times K)$ onto the first coordinate of $K \times K$ is an affine 2-transitive subgroup of $K$. Since $G^{1}$ has degree $p$, it is therefore isomorphic to $\operatorname{AGL}_{1}(p)$. In particular, $G^{1}$ has order $p(p-1)$. By [49, Table 2], no such subgroup of $K$ exists when $(K, p)$ is one of $\left(\mathrm{PSL}_{2}(11), 11\right),\left(M_{11}, 11\right)$ or $\left(M_{23}, 23\right)$, so $T=\operatorname{PSL}_{m}(q)$ and $K \leqslant \mathrm{P \Gamma L}_{m}(q)$ for some prime power $q$ and $m \geqslant 2$ such that $p=\left(q^{m}-1\right) /(q-1)$, and $G^{1}$ is a subgroup of the normaliser of a Singer cycle. Now $q=r^{f}$ for some prime $r$, and it follows from [31, p.187] that the normaliser of a Singer cycle in $\mathrm{P}_{m}(q)$ has order $\left(q^{m}-1\right) /(q-1) f m=p f m$. Since $G^{1}$ has order $p(p-1)$, we conclude that $p-1$ divides $f m$. In particular,

$$
f m \geqslant p-1=\left(q^{m}-1\right) /(q-1)-1 \geqslant q^{m-1} \geqslant 2^{f(m-1)} \geqslant 2 f(m-1) .
$$


Thus $m=2$, and all of the inequalities above are equalities. Since $q \neq 2$ (or else $T$ is soluble), we conclude that $q=4$. Hence $T \simeq A_{5}$ and $p=5$, and as we saw above, (i) holds.

\section{The AFFINE PRIMITIVE PERMUTATION GROUPS OF RANK 3}

In this section, we state and prove a modified version of Liebeck's classification [39] of the affine primitive permutation groups of rank 3 . In particular, we provide more details about the rank 3 groups $G$ when $G_{0}$ is imprimitive or stabilises a tensor product decomposition. Throughout this section, recall the definition of $\zeta_{q}$ and $\sigma_{q}$ from $\S 2.3$.

Theorem 3.1 ([39]). Let $G$ be a finite affine primitive permutation group of rank 3 with socle $V:=V_{d}(p)$ where $d \geqslant 1$ and $p$ is prime. Then $G_{0}$ belongs to one of the following classes.

(T) The tensor product classes. Here $G_{0}$ stabilises a decomposition $V=V_{2}(q) \otimes V_{m}(q)$ where $q^{2 m}=p^{d}$ and $m \geqslant 2$, and one of the following holds.

(1) $\mathrm{SL}_{2}(q) \otimes \mathrm{SL}_{m}(q) \unlhd G_{0}$ where $q \geqslant 4$ or $(m, q)=(2,3)$.

(2) $\mathrm{SL}_{2}(5) \otimes \mathrm{SL}_{m}(q) \unlhd G_{0}$ where $q \in\{9,11,19,29,59\}$.

(3) $1 \otimes \mathrm{SL}_{m}(q) \unlhd G_{0} \leqslant N_{\mathrm{GL}_{2}(q)}\left(\mathrm{SL}_{2}(3)\right) \otimes \mathrm{GL}_{m}(q)$ where $q \in\{3,5,7,11,23\}$ but $G_{0} \nless$ $\Gamma \mathrm{L}_{1}\left(q^{2}\right) \otimes \mathrm{GL}_{m}(q)$. Further, $-1 \otimes g \in G_{0}$ for some $g \in \mathrm{GL}_{m}(q)$.

(S) The subfield classes. Here $q^{2 n}=p^{d}$ and one of the following holds.

(0) $V=V_{2}\left(q^{n}\right)$ and $\mathrm{SL}_{2}(q) \unlhd G_{0} \leqslant \Gamma L_{2}\left(q^{n}\right)$ where $n=3$.

(1) $V=V_{n}\left(q^{2}\right)$ and $\mathrm{SL}_{n}(q) \unlhd G_{0} \leqslant \Gamma \mathrm{L}_{n}\left(q^{2}\right)$ where $n \geqslant 2$.

(2) $V=V_{n}\left(q^{2}\right)$ and $A_{7} \unlhd G_{0} \leqslant \Gamma L_{n}\left(q^{2}\right)$ where $A_{7} \leqslant \mathrm{SL}_{4}(2) \simeq A_{8}$ and $(n, q)=(4,2)$.

(I) The imprimitive classes. Here $G_{0}$ stabilises a decomposition $V=V_{n}(p) \oplus V_{n}(p)$ where $2 n=d$. Further, $G_{0} \leqslant \Gamma L_{m}(q) \backslash S_{2}$ where $q^{m}=p^{n}$ and one of the following holds.

(0) $G_{0} \leqslant \Gamma L_{1}(q) \imath S_{2}$ and $m=1$.

(1) $\mathrm{SL}_{m}(q) \times \mathrm{SL}_{m}(q) \unlhd G_{0}$ where $m \geqslant 2$ and $(m, q) \neq(2,2),(2,3)$.

(2) $\operatorname{Sp}_{m}(q) \times \operatorname{Sp}_{m}(q) \unlhd G_{0}$ where $m$ is even and $m \geqslant 4$.

(3) $G_{2}(q)^{\prime} \times G_{2}(q)^{\prime} \unlhd G_{0}$ where $m=6$ and $q$ is even.

(4) $S \times S \unlhd G_{0} \leqslant N_{\Gamma \mathrm{L}_{m}(q)}(S) \imath S_{2}$ where $S=\mathrm{SL}_{2}(5), m=2$ and $q \in\{9,11,19,29,59\}$.

(5) $G_{0}=A_{6} 2 S_{2}$ or $A_{7}$ 々 $S_{2}$ where $m=4$ and $q=2$.

(6) $G_{0}=\mathrm{SL}_{2}(13)$ 乙 $S_{2}$ where $m=6$ and $q=3$.

(7) $G_{0} \leqslant N_{\mathrm{GL}_{m}(q)}\left(\mathrm{SL}_{2}(3)\right)<S_{2}$ where $m=2$ and $q \in\{3,5,7,11,23\}$.

(8) $E \times E \unlhd G_{0} \leqslant N_{\mathrm{GL}_{m}(q)}(E) \imath S_{2}$ where $E=D_{8} \circ Q_{8}, m=4$ and $q=3$.

$(\mathrm{R})$ The remaining infinite classes.

(0) $G_{0} \leqslant \Gamma L_{1}\left(p^{d}\right)$.

(1) $\mathrm{SU}_{n}(q) \unlhd G_{0}$ where $q^{2 n}=p^{d}$ and $n \geqslant 3$.

(2) $\Omega_{2 m}^{\varepsilon}(q) \unlhd G_{0}$ where $q^{2 m}=p^{d}$ and either $m \geqslant 3$ and $\varepsilon= \pm$, or $m=2$ and $\varepsilon=-$.

(3) $V=\bigwedge^{2}\left(V_{5}(q)\right)$ and $\mathrm{SL}_{5}(q) \unlhd G_{0}$ where $q^{10}=p^{d}$.

(4) $\mathrm{Sz}(q)={ }^{2} B_{2}(q) \unlhd G_{0}$ where $q^{4}=p^{d}$ and $q=2^{2 n+1}$ where $n \geqslant 1$.

(5) $V=V_{n}(q)$ is a spin module and $\operatorname{Spin}_{m}^{\varepsilon}(q) \unlhd G_{0}$ where $q^{n}=p^{d}$ and $(m, n, \varepsilon)$ is one of $(7,8, \circ)$ or $(10,16,+)$.

(E) The extraspecial class. Here $E \unlhd G_{0}$ and $E$ is irreducible on $V_{d}(p)$ where $\left(E, p^{d}, G\right)$ is given by Table 2.

(AS) The almost simple class. Here $S \unlhd G_{0} \leqslant \Gamma \mathrm{L}_{n}(q)$ and $S$ is irreducible on $V_{d}(p)$ where $\left(S, p^{d}, G\right)$ is given by Table $3, q^{n}=p^{d}$, and $q$ is given by the embedding of $S$ in $\operatorname{SL}_{n}(q)$.

Throughout this paper, we say that an affine permutation group $G$ or its stabiliser $G_{0}$ belongs to (or lies in, is contained in, etc.) one of the classes (T1)-(T3), (S0)-(S2), (I0)-(I8), (R0)-(R5), (E) or (AS) if $G$ has rank 3 and $G_{0}$ satisfies the given description. We also say that $G$ or $G_{0}$ belongs to class (T), (S), (I) or (R), respectively. We remark that if $G$ belongs to one of the classes (T1)-(T3), (S0)-(S2), (I0)-(I8) with $p^{n}>2$, (R1)-(R5), (E) or (AS), then $G$ is primitive: this holds for classes (E) and (AS) by Lemma 2.1 and can otherwise be verified using Lemma 2.2 and the descriptions of the orbits of $G_{0}$ that are given in subsequent sections. 


\begin{tabular}{cccc}
\hline$E$ & $p^{d}$ & Subdegrees of $G$ & Conditions \\
\hline & $7^{2}$ & 24,24 & $G_{0} \nless \Gamma L_{1}\left(7^{2}\right)$ \\
& $13^{2}$ & 72,96 & \\
$Q_{8}$ & $17^{2}$ & 96,192 & \\
& $19^{2}$ & 144,216 & \\
& $23^{2}$ & 264,264 & \\
& $29^{2}$ & 168,672 & \\
& $31^{2}$ & 240,720 & \\
& $47^{2}$ & 1104,1104 & \\
\hline $3^{1+2}$ & $2^{6}$ & 27,36 & $\mathrm{SU}_{3}(2) \nless G_{0} \leqslant \Gamma \mathrm{U}_{3}(2)$ \\
$D_{8} \circ Q_{8}$ & $5^{4}$ & 240,384 & \\
$D_{8} \circ Q_{8} \circ\left\langle\zeta_{5}\right\rangle$ & $5^{4}$ & 240,384 & $G_{0} \nless N_{\mathrm{GL}_{4}(5)}\left(D_{8} \circ Q_{8}\right)$ \\
$D_{8} \circ Q_{8}$ & $7^{4}$ & 480,1920 & \\
$Q_{8} \circ Q_{8} \circ Q_{8}$ & $3^{8}$ & 1440,5120 & \\
\hline \multicolumn{4}{c}{ TABLE 2. Extraspecial class $(\mathrm{E})$}
\end{tabular}

\begin{tabular}{cccc}
\hline$S$ & $p^{d}$ & Embedding of $S$ & Subdegrees of $G$ \\
\hline & $3^{4}$ & & 40,40 \\
& $31^{2}$ & & 360,600 \\
& $41^{2}$ & & 480,1200 \\
$2 \cdot A_{5}$ & $7^{4}$ & $S<\mathrm{SL}_{2}\left(p^{d / 2}\right)$ & 960,1440 \\
& $71^{2}$ & & 840,4200 \\
& $79^{2}$ & & 1560,4680 \\
& $89^{2}$ & & 2640,5280 \\
\hline $3 \cdot A_{6}$ & $2^{6}$ & $S<\mathrm{SL}_{3}(4)$ & 18,45 \\
$2 \cdot A_{6}$ & $5^{4}$ & $S<\mathrm{Sp}_{4}(5)$ & 144,480 \\
$2 \cdot A_{7}$ & $7^{4}$ & $S<\mathrm{Sp}_{4}(7)$ & 720,1680 \\
$A_{9}$ & $2^{8}$ & $S<\Omega_{8}^{+}(2)$ & 120,135 \\
$A_{10}$ & $2^{8}$ & $S<\mathrm{Sp}_{8}(2)$ & 45,210 \\
$\mathrm{PSL}_{2}(17)$ & $2^{8}$ & $S<\mathrm{Sp}_{8}(2)$ & 102,153 \\
$2 \cdot \mathrm{PSL}_{3}(4)$ & $3^{6}$ & $S<\Omega_{6}^{-}(3)$ & 224,504 \\
$2 \cdot \mathrm{PSU}_{4}(2)$ & $7^{4}$ & $S<\mathrm{SL}_{4}(7)$ & 240,2160 \\
$M_{11}$ & $3^{5}$ & $S<\mathrm{SL}_{5}(3)$ & 22,220 \\
& & & 110,132 \\
$M_{24}$ & $2^{11}$ & $S<\mathrm{SL}_{11}(2)$ & 276,1771 \\
& & & 759,1288 \\
$2 \cdot \mathrm{Suz}_{2}$ & $3^{12}$ & $S<\mathrm{Sp}_{12}(3)$ & 65520,465920 \\
$2 \cdot G_{2}(4)$ & $3^{12}$ & $S<2 \cdot \mathrm{Suz}_{2}<\mathrm{Sp}_{12}(3)$ & 65520,465920 \\
$J_{2}$ & $2^{12}$ & $S<G_{2}(4)<\mathrm{Sp}_{6}(4)$ & 1575,2520 \\
$2 \cdot J_{2}$ & $5^{6}$ & $S<\mathrm{Sp}_{6}(5)$ & 7560,8064 \\
\hline & $\mathrm{TABLE} 3$. & $\mathrm{Almost} \operatorname{simple}$ class $(\mathrm{AS})$ \\
& & &
\end{tabular}

In Table 4, we list the subdegrees of the groups in classes (T), (S), (I) and (R), as given by [39, Table 12]. Note that the groups in the classes (S0), (I0) and (R0) are precisely those that are listed in Theorem 1.4(iv). We caution the reader that the groups in class (I) are not necessarily 


\begin{tabular}{lll}
\hline Class of $G$ & $p^{d}$ & Subdegrees of $G$ \\
\hline (T): tensor product & $q^{2 m}$ & $(q+1)\left(q^{m}-1\right), q\left(q^{m}-1\right)\left(q^{m-1}-1\right)$ \\
(S): subfield & $q^{2 n}$ & $(q+1)\left(q^{n}-1\right), q\left(q^{n}-1\right)\left(q^{n-1}-1\right)$ \\
(I): $G_{0}$ imprimitive & $p^{2 n}$ & $2\left(p^{n}-1\right),\left(p^{n}-1\right)^{2}$ \\
(R0): $G_{0} \leqslant \Gamma L_{1}\left(p^{d}\right)$ & $p^{d}$ & given in $[25, \S 3]$ \\
(R1): $\operatorname{SU}_{n}(q) \unlhd G_{0}$ & $q^{2 n}$ & $\left(q^{n}-1\right)\left(q^{n-1}+1\right), q^{n-1}(q-1)\left(q^{n}-1\right), n$ even \\
& & $\left(q^{n}+1\right)\left(q^{n-1}-1\right), q^{n-1}(q-1)\left(q^{n}+1\right), n$ odd \\
& & $\left.q^{m}-1\right)\left(q^{m-1}+1\right), q^{m-1}(q-1)\left(q^{m}-1\right), \varepsilon=+$ \\
(R2): $\Omega_{2 m}^{\varepsilon}(q) \unlhd G_{0}$ & $q^{2 m}$ & \\
& & $\left(q^{m}+1\right)\left(q^{m-1}-1\right), q^{m-1}(q-1)\left(q^{m}+1\right), \varepsilon=-$ \\
(R3): $\operatorname{SL}_{5}(q) \unlhd G_{0}$ & $q^{10}$ & $\left(q^{5}-1\right)\left(q^{2}+1\right), q^{2}\left(q^{5}-1\right)\left(q^{3}-1\right)$ \\
(R4): $\operatorname{Sz}(q) \unlhd G_{0}$ & $q^{4}$ & $\left(q^{2}+1\right)(q-1), q\left(q^{2}+1\right)(q-1)$ \\
(R5): $\operatorname{Spin}_{m}^{\varepsilon}(q) \unlhd G_{0}$ & $q^{8}$ & $\left(q^{4}-1\right)\left(q^{3}+1\right), q^{3}\left(q^{4}-1\right)(q-1),(m, \varepsilon)=(7, \circ)$ \\
& $q^{16}$ & $\left(q^{8}-1\right)\left(q^{3}+1\right), q^{3}\left(q^{8}-1\right)\left(q^{5}-1\right),(m, \varepsilon)=(10,+)$ \\
\hline
\end{tabular}

TABLE 4. Classes (T), (S), (I) and (R)

subgroups of $\Gamma \mathrm{L}_{2 m}(q)$. For example, if $q$ is not prime, then $\Gamma \mathrm{L}_{m}(q)>S_{2}$ lies in class (I0) or (I1) but is not a subgroup of $\Gamma L_{2 m}(q)$ (see $\S 13$ for more details). We also caution the reader that (R5) includes the case where $m=7$ and $q$ is even, in which case $\operatorname{Spin}_{7}(q) \simeq \operatorname{Sp}_{6}(q)$.

In order to prove Theorem 3.1, we first consider the case where $G_{0}$ stabilises a tensor product decomposition. In the following, recall the definition of $\sigma_{q}$ from $\S 2.3$.

Theorem 3.2. Let $G$ be an affine permutation group of rank 3 on $V$ where $G_{0}$ stabilises a decomposition $V=V_{2}(q) \otimes V_{m}(q)$ for some $m \geqslant 2$ and prime power $q$. Then either $G_{0}$ belongs to one of the classes (R0), (I0), (T1), (T2) or (T3), or one of the following holds.

(T4) $1 \otimes A_{7} \unlhd G_{0}$ where $(m, q)=(4,2)$.

(T5) $1 \otimes \mathrm{SL}_{m}(q) \unlhd G_{0} \leqslant\left(\mathrm{GL}_{1}\left(q^{2}\right) \otimes \mathrm{GL}_{m}(q)\right):\left\langle(t \otimes 1) \sigma_{q}\right\rangle$ for some $t \in \mathrm{GL}_{2}(q)$ where $\Gamma L_{1}\left(q^{2}\right)=$ $\mathrm{GL}_{1}\left(q^{2}\right):\left\langle t \sigma_{q}\right\rangle$.

(T6) $\mathrm{SL}_{2}(q) \otimes 1 \unlhd G_{0} \leqslant\left(\mathrm{GL}_{2}(q) \otimes \mathrm{GL}_{1}\left(q^{3}\right)\right):\left\langle(1 \otimes t) \sigma_{q}\right\rangle$ for some $t \in \mathrm{GL}_{m}(q)$ where $m=3$ and $\Gamma \mathrm{L}_{1}\left(q^{3}\right)=\mathrm{GL}_{1}\left(q^{3}\right):\left\langle t \sigma_{q}\right\rangle$.

We will see in the proof of Theorem 3.1 that if $G_{0}$ satisfies (T4), (T5) or (T6), then $G_{0}$ lies in class (S2), (S1) or (S0), respectively, and we will see in $\S 11, \S 12$ and $\S 18$ that these two different viewpoints of $G_{0}$ are important. Throughout this paper, we say that an affine permutation group $G$ or its stabiliser $G_{0}$ belongs to (or lies in, is contained in, etc.) one of the classes (T4)-(T6) if $G$ has rank 3 and $G_{0}$ satisfies the given description. We also enlarge the class (T) so that it contains the groups in classes (T4)-(T6). Note that the statement of Theorem 3.2 is similar to that of [4, Lemma 55], but [4, Lemma 55] is proved as part of an analysis of $2-(v, k, 1)$ designs admitting rank 3 automorphism groups.

Proof of Theorem 3.2. Let $U:=V_{2}(q), W:=V_{m}(q)$ and $A:=(\operatorname{GL}(U) \otimes \operatorname{GL}(W)): \operatorname{Aut}\left(\mathbb{F}_{q}\right)$ (see §2.3). We write elements of $A$ as $g \otimes h$ for $g \in \Gamma L(U)$ and $h \in \Gamma L(W)$ where $g$ and $h$ are $\sigma$-semilinear for some $\sigma \in \operatorname{Aut}\left(\mathbb{F}_{q}\right)$. Let $H_{0}:=A \cap G_{0}$. Either $H_{0}=G_{0}$, or $m=2$ and $G_{0}=H_{0}\left\langle\left(g_{0} \otimes h_{0}\right) \tau\right\rangle$ for some $g_{0}, h_{0} \in \Gamma \mathrm{L}(U)$ where $\tau \in \mathrm{GL}(V)$ is defined by $u \otimes w \mapsto w \otimes u$ for all $u, w \in U=W$; let $\alpha:=\left(g_{0} \otimes h_{0}\right) \tau$. The orbits of $G_{0}$ on $V^{*}$ are the simple tensors $\{u \otimes w$ : $\left.u \in U^{*}, w \in W^{*}\right\}$ and the non-simple tensors $\left\{u_{1} \otimes w_{1}+u_{2} \otimes w_{2}: w_{1}, w_{2} \in W, \operatorname{dim}\left\langle w_{1}, w_{2}\right\rangle=2\right\}$ for any choice of basis $\left\{u_{1}, u_{2}\right\}$ of $U$. Let $p$ and $d$ be such that $q^{2 m}=p^{d}$ where $p$ is prime.

Let $Z_{U}:=Z(\mathrm{GL}(U)), Z_{W}:=Z(\mathrm{GL}(W)), Z_{V}:=Z(\mathrm{GL}(V))$ and $Z_{0}:=Z_{V} \cap G_{0}=Z_{V} \cap H_{0}$. Define $G_{0}^{U}$ to be the set of $g \in \Gamma \mathrm{L}(U)$ for which there exists $h \in \Gamma \mathrm{L}(W)$ such that $g$ and $h$ are both $\sigma$-semilinear for some $\sigma \in \operatorname{Aut}\left(\mathbb{F}_{q}\right)$ and $g \otimes h \in H_{0}$. Define $G_{0}^{W}$ similarly. Then $G_{0}^{U} \leqslant \Gamma L(U)$ and $G_{0}^{W} \leqslant \Gamma \mathrm{L}(W)$. Define $\varphi_{U}: A \rightarrow \operatorname{P\Gamma L}(U)$ by $g \otimes h \mapsto Z_{U} g$. Define $\varphi_{W}$ similarly. Then 
$\varphi_{U}$ and $\varphi_{W}$ are homomorphisms with kernels $1 \otimes \mathrm{GL}(W)$ and $\mathrm{GL}(U) \otimes 1$, respectively. Let $K_{U}:=\operatorname{ker}\left(\varphi_{U}\right) \cap H_{0}$ and $K_{W}:=\operatorname{ker}\left(\varphi_{W}\right) \cap H_{0}$. Note that $G_{0}^{U} Z_{U} / Z_{U}=H_{0} \varphi_{U} \simeq H_{0} / K_{U}$ and $G_{0}^{W} Z_{W} / Z_{W}=H_{0} \varphi_{W} \simeq H_{0} / K_{W}$. Now $K_{U}=1 \otimes H_{W}$ for some $H_{W} \leqslant \operatorname{GL}(W)$ and $K_{W}=H_{U} \otimes 1$ for some $H_{U} \leqslant \operatorname{GL}(U)$. Note that $H_{U} \unlhd G_{0}^{U}$ and $H_{W} \unlhd G_{0}^{W}$.

First we claim that if $H_{0} \neq G_{0}$, then $G_{0}^{U}$ and $G_{0}^{W}$ are conjugate in $\Gamma \mathrm{L}(U)$. If $g \in G_{0}^{U}$, then $g \otimes h \in H_{0}$ for some $h \in \Gamma \mathrm{L}(U)$, and $h^{h_{0}} \otimes g^{g_{0}}=(g \otimes h)^{\alpha} \in H_{0}$, so $\left(G_{0}^{U}\right)^{g_{0}} \leqslant G_{0}^{W}$. Similarly, $\left(G_{0}^{W}\right)^{h_{0}} \leqslant G_{0}^{U}$. By comparing orders, it follows that $\left(G_{0}^{U}\right)^{g_{0}}=G_{0}^{W}$.

Next we claim that $G_{0}^{U}$ is transitive on $\operatorname{PG}(U)$ and $G_{0}^{W}$ is transitive on $\operatorname{PG}(W)$. Since $G_{0}$ is transitive on the set of simple tensors of $V$, the claim holds when $G_{0}=H_{0}$. Suppose then that $G_{0} \neq H_{0}$. Now $G_{0}^{U}$ and $G_{0}^{W}$ have the same number of orbits, say $r$, on $\mathrm{PG}(U)$ since they are conjugate, so $H_{0}$ has at least $r^{2}$ orbits on the set of simple tensors of $V$. On the other hand, $G_{0}$ is transitive on the set of simple tensors and $\left[G_{0}: H_{0}\right]=2$, so $H_{0}$ has at most 2 orbits on the set of simple tensors. Thus $r=1$, as desired.

Case 1: $G_{0}$ is soluble. We may assume that $G_{0}$ does not belong to class (I0) or (R0). Then $(m, q) \neq(2,2)$, or else $G_{0} \leqslant \mathrm{GL}_{2}(2) \curlywedge S_{2}=\Gamma \mathrm{L}_{1}(4) \curlywedge S_{2}$. If $G_{0}$ stabilises a decomposition $V=V_{\ell}(p) \oplus V_{\ell}(p)$ where $2 \ell=d$, then $q^{2 m}=p^{2 \ell}$ and $G_{0}$ has orbit sizes $2\left(p^{\ell}-1\right)$ and $\left(p^{\ell}-1\right)^{2}$ on $V^{*}$, but $G_{0}$ also has orbit sizes $(q+1)\left(q^{m}-1\right)$ and $q\left(q^{m}-1\right)\left(q^{m-1}-1\right)$, so $(m, q)=(2,2)$, a contradiction. By [24, Theorem 1.1] and a consideration of the suborbit lengths of $G$, we conclude that $(m, q)=(2,3)$ or $(3,3)$. Using MAGMA, we determine that if $(m, q)=(2,3)$, then $G_{0}$ belongs to class (T1) or (T5), and if $(m, q)=(3,3)$, then either $\mathrm{GL}(U) \otimes \mathrm{GL}_{1}\left(q^{3}\right) \unlhd G_{0} \leqslant \mathrm{GL}(U) \otimes \Gamma \mathrm{L}_{1}\left(q^{3}\right)$, in which case $G_{0}$ belongs to (T6), or $G_{0}=\Gamma \mathrm{L}_{1}\left(q^{2}\right) \otimes \Gamma \mathrm{L}_{1}\left(q^{3}\right) \leqslant \Gamma \mathrm{L}_{1}\left(q^{6}\right)$, a contradiction.

Case 2: $G_{0}$ is insoluble. Now $G_{0}^{U}$ and $G_{0}^{W}$ are not both soluble, for $K_{U} / Z_{0}=K_{U} /\left(K_{U} \cap\right.$ $\left.K_{W}\right) \simeq K_{U} K_{W} / K_{W} \unlhd H_{0} / K_{W} \simeq G_{0}^{W} Z_{W} / Z_{W}$, and similarly $K_{W} / Z_{0}$ is isomorphic to a normal subgroup of $H_{0} / K_{U} \simeq G_{0}^{U} Z_{U} / Z_{U}$, so if $G_{0}^{U}$ and $G_{0}^{W}$ are both soluble, then $K_{W}$ and $K_{U}$ are both soluble, but then $H_{0}$ is soluble, so $G_{0}$ is soluble, a contradiction.

If $m \geqslant 4$, then $G_{0}^{W}$ is transitive on the lines of $\mathrm{PG}(W)$ since $G_{0}$ is transitive on the set of non-simple tensors of $V$, so either $G_{0}^{W}$ is 2-transitive on $\mathrm{PG}(W)$, or $G_{0}^{W}=\Gamma L_{1}\left(2^{5}\right)$ with $m=5$ and $q=2$ [35]. In this latter case, both $G_{0}^{U}$ and $G_{0}^{W}$ are soluble, a contradiction. Hence $G_{0}^{W}$ is 2-transitive on $\mathrm{PG}(W)$, so either $\mathrm{SL}(W) \unlhd G_{0}^{W}$, or $G_{0}^{W}=A_{7}$ with $m=4$ and $q=2$ [12]. If instead $m \leqslant 3$ and $G_{0}^{W}$ is insoluble, then since $G_{0}^{W} Z_{W}$ is transitive on $W^{*}$, either $\operatorname{SL}(W) \unlhd G_{0}^{W}$, or $\mathrm{SL}_{2}(5) \unlhd G_{0}^{W}$ with $m=2$ and $q \in\{9,11,19,29,59\}$ by Theorem 2.18. Thus one of the following holds: (a) $\mathrm{SL}(W) \unlhd G_{0}^{W}$ where $m \geqslant 2$ and $(m, q) \neq(2,2)$ or $(2,3)$; (b) $\mathrm{SL}_{2}(5) \unlhd G_{0}^{W}$ where $m=2$ and $q \in\{9,11,19,29,59\}$; (c) $G_{0}^{W}$ is soluble where $m=2$ or $3 ;$ (d) $G_{0}^{W}=A_{7}$ where $m=4$ and $q=2$. Further, since $G_{0}^{U} Z_{U}$ is transitive on $U^{*}$, either $\operatorname{SL}(U) \unlhd G_{0}^{U}$, or $\mathrm{SL}_{2}(5) \unlhd G_{0}^{U}$ where $q \in\{9,11,19,29,59\}$, or $G_{0}^{U}$ is soluble.

Suppose for a contradiction that $H_{W} \leqslant Z_{W}$. Then $K_{U}=Z_{0}$, so $H_{0} / Z_{0} \simeq G_{0}^{U} Z_{U} / Z_{U} \leqslant$ $\operatorname{P\Gamma L}(U)$. First suppose that $G_{0}=H_{0}$. Now $(q+1)\left(q^{m}-1\right)$ divides $\left|G_{0}\right|$, so $(q+1)\left(q^{m}-1\right)$ divides $q(q-1)\left(q^{2}-1\right) e$ where $q=p^{e}$. Then $q^{m-1}+\cdots+q+1$ divides $q(q-1) e$ and therefore $(q-1) e$. But $q \geqslant e$, so $q^{m-1}+\cdots+q+1>(q-1) e$ for $m \geqslant 3$, a contradiction. Thus $m=2$. Since $(q+1, q-1)=1$ or 2 , it follows that $q+1$ divides $2 e$, a contradiction. Now suppose that $G_{0} \neq H_{0}$. Then $(q+1)\left(q^{2}-1\right)$ divides $\left|G_{0}\right|=2\left|H_{0}\right|$, so $q+1$ divides $2(q-1) e$. If $q$ is even, then $q+1$ divides $e$, a contradiction. Thus $q$ is odd, and $q+1$ divides $4 e$, so $q=3$, but then $G_{0}^{U}$ and $G_{0}^{W}$ are soluble, a contradiction.

Thus $H_{W}$ is a non-central normal subgroup of $G_{0}^{W}$. If (d) holds, then $H_{W}=A_{7}$, so $G_{0}$ lies in class (T4). Hence we may assume that one of (a), (b) or (c) holds. Since $G_{0}^{W} Z_{W} / Z_{W}$ is almost simple when (a) or (b) holds, $\mathrm{SL}(W) \unlhd H_{W}$ when (a) holds and $\mathrm{SL}_{2}(5) \unlhd H_{W}$ when (b) holds.

If $H_{U} \leqslant Z_{U}$, then $K_{W}=Z_{0}$, so $G_{0}^{W} Z_{W} / Z_{W} \simeq H_{0} / Z_{0} \unrhd K_{U} / Z_{0} \simeq H_{W} Z_{W} / Z_{W}$, and since the quotient of $G_{0}^{W} Z_{W} / Z_{W}$ by $H_{W} Z_{W} / Z_{W}$ is soluble, it follows that $H_{0} / K_{U}$ is soluble and therefore that $G_{0}^{U}$ is soluble. Hence when $G_{0}^{U}$ is insoluble, either $\mathrm{SL}(U) \unlhd H_{U}$ and $q \geqslant 4$, or $\mathrm{SL}_{2}(5) \unlhd H_{U}$ where $q \in\{9,11,19,29,59\}$. 
If $G_{0} \neq H_{0}$, then since $G_{0}^{U}$ and $G_{0}^{W}$ are conjugate, we may assume that $S \otimes S \unlhd H_{0}$ where either $S=\mathrm{SL}(U)$, or $S=\mathrm{SL}_{2}(5)$ and $q \in\{9,11,19,29,59\}$. Now $S$ is the unique subgroup of $G_{0}^{U}$ and $G_{0}^{W}$ that is isomorphic to $S$, so $(S \otimes S)^{\alpha}=S^{h_{0}} \otimes S^{g_{0}}=S \otimes S$. Thus $S \otimes S \unlhd G_{0}$.

We conclude that one of the following holds: (i) $\mathrm{SL}(U) \otimes \mathrm{SL}(W) \unlhd G_{0}$ where $q \geqslant 4$; (ii) $\mathrm{SL}_{2}(5) \otimes \mathrm{SL}(W) \unlhd G_{0}=H_{0}$ where $q \in\{9,11,19,29,59\} ; \quad$ (iii) $1 \otimes \mathrm{SL}(W) \unlhd G_{0}=H_{0}$ and $G_{0}^{U}$ is soluble; (iv) $\mathrm{SL}(U) \otimes \mathrm{SL}_{2}(5) \unlhd G_{0}=H_{0}$ where $m=2$ and $q \in\{9,11,19,29,59\}$; (v) $\mathrm{SL}_{2}(5) \otimes \mathrm{SL}_{2}(5) \unlhd G_{0}$ where $m=2$ and $q \in\{9,11,19,29,59\} ;(\mathbf{v i}) 1 \otimes \mathrm{SL}_{2}(5) \unlhd G_{0}=H_{0}$ and $G_{0}^{U}$ is soluble where $m=2$ and $q \in\{9,11,19,29,59\}$; (vii) $\mathrm{SL}(U) \otimes 1 \unlhd G_{0}=H_{0}$ and $G_{0}^{W}$ is soluble where $m=2$ or 3 ; (viii) $\mathrm{SL}_{2}(5) \otimes 1 \unlhd G_{0}=H_{0}$ and $G_{0}^{W}$ is soluble where $m=2$ or 3 and $q \in\{9,11,19,29,59\}$. Moreover, if $m \leqslant 3$ and $G_{0}^{W}$ is soluble, then since $G_{0}^{W} Z_{W}$ is transitive on $W^{*}$, either $G_{0}^{W} \leqslant \Gamma L_{1}\left(q^{m}\right)$, or $\mathrm{SL}_{2}(3) \unlhd G_{0}^{W} Z_{W}$ where $m=2$ and $q \in\{3,5,7,11,23\}$ by Theorem 2.18. A similar result applies to $G_{0}^{U}$.

Cases (i), (ii), (iv) and (v). If (i) or (ii) holds, then $G_{0}$ lies in class (T1) or (T2), respectively. If (iv) holds, then $G_{0}$ lies in class (T2) by interchanging $U$ and $W$. Suppose that (v) holds. If $q \neq 9$, then $G_{0} \leqslant\left(\mathrm{SL}_{2}(5) \otimes \mathrm{SL}_{2}(5) Z_{V}\right) .2$, but $q$ divides $\left|G_{0}\right|$, a contradiction. Thus $q=9$, so $G_{0} \leqslant\left(\mathrm{SL}_{2}(5) \otimes \mathrm{SL}_{2}(5) Z_{V}\right) \cdot 2^{2}$, but, using MAGMA, we determine that this group is not transitive on the set of non-simple tensors of $V$, a contradiction.

Case (vi). Either $G_{0}^{U} \leqslant \Gamma L_{1}\left(q^{2}\right)$, or $\mathrm{SL}_{2}(3) \unlhd G_{0}^{U} Z_{U}$ and $q=11$, in which case $G_{0}^{U} \leqslant$ $\mathrm{GL}_{2}(3) Z_{U}$. In particular, the order of $G_{0}^{U}$ divides $\left(q^{2}-1\right) 2 e$, or 240, respectively. Further, $\mathrm{SL}_{2}(5) \unlhd H_{W} \leqslant \mathrm{GL}(W)$, so $H_{W} \leqslant \mathrm{SL}_{2}(5) \circ Z_{W}$. In particular, $1 \otimes H_{W}=K_{U}$ has order dividing $60(q-1)$. Recall that $G_{0}^{U} Z_{U} / Z_{U} \simeq G_{0} / K_{U}$, and $q$ divides $\left|G_{0}\right|$. If $q \neq 9$, then $q$ divides $\left|G_{0}^{U}\right|$, a contradiction. Similarly, if $q=9$, then 3 divides $\left|G_{0}^{U}\right|$, a contradiction.

Case (viii). We have just proved that $m \neq 2$, so $m=3$ and $G_{0}^{W} Z_{W} \leqslant \Gamma L_{1}\left(q^{3}\right)$. In particular, the order of $G_{0}^{W} Z_{W}$ divides $\left(q^{3}-1\right) 3 e$. Further, $\mathrm{SL}_{2}(5) \unlhd H_{U} \leqslant \mathrm{GL}(U)$, so $H_{U} \otimes 1=K_{W}$ has order dividing $60(q-1)$. Recall that $G_{0}^{W} Z_{W} / Z_{W} \simeq G_{0} / K_{W}$, and $q$ divides $\left|G_{0}\right|$. If $q \neq 9$, then $q$ divides $\left|G_{0}^{W}\right|$, a contradiction. Thus $q=9$. Now $q\left(q^{2}-1\right)\left(q^{3}-1\right)(q-1)$ divides $\left|G_{0}\right|\left|Z_{W}\right|$, which divides $\left(q^{3}-1\right) 3 e \cdot 60(q-1)$, a contradiction.

Case (iii). Either $\mathrm{SL}_{2}(3) \unlhd G_{0}^{U} Z_{U}$ where $q \in\{3,5,7,11,23\}$ and $G_{0}^{U} \nless \Gamma_{1}\left(q^{2}\right)$, or $G_{0}^{U} \leqslant$ $\Gamma L_{1}\left(q^{2}\right) \leqslant \Gamma \mathrm{L}(U)$. If the former holds, then $G_{0} \leqslant\left(N_{\mathrm{GL}(U)}\left(\mathrm{SL}_{2}(3)\right)\right) \otimes \mathrm{GL}(W)$, and $-1 \in G_{0}^{U}$ since $Q_{8}=\mathrm{SL}_{2}(3)^{\prime} \leqslant G_{0}^{U}$ and $Q_{8}$ is irreducible on $U$. Thus $-1 \otimes g \in G_{0}$ for some $g \in \mathrm{GL}(W)$ and $G_{0}$ lies in class (T3). Otherwise, since $\Gamma_{1}\left(q^{2}\right)=\mathrm{GL}_{1}\left(q^{2}\right):\left\langle t \sigma_{q}\right\rangle$ for some $t \in \mathrm{GL}(U)$, it follows that $G_{0}$ lies in class (T5).

Case (vii). If $m=2$, then $G_{0}$ lies in class (T5) by interchanging $U$ and $W$, so we may assume that $m=3$. Now $G_{0}^{W} \leqslant \Gamma L_{1}\left(q^{3}\right) \leqslant \Gamma \mathrm{L}(W)$, so $G_{0}$ lies in class (T6).

Next we consider the case where $G_{0}$ is imprimitive. Note that our result in this case is similar to [4, Proposition 39], but [4, Proposition 39] is proved as part of an analysis of $2-(v, k, 1)$ designs admitting rank 3 automorphism groups and omits the cases where $G_{0}$ is soluble or $(n, p)=(6,2)$.

Theorem 3.3. Let $G$ be an affine permutation group of rank 3 on $V$ where $G_{0}$ stabilises a decomposition $V=V_{n}(p) \oplus V_{n}(p)$ for some $n \geqslant 1$ and prime $p$. Then $G_{0}$ belongs to one of the classes (I0)-(I8).

Proof. The group $G_{0}$ is a subgroup of $\mathrm{GL}_{n}(p) \imath\langle\tau\rangle$ where $\tau$ is the involution in $\mathrm{GL}_{2 n}(p)$ defined by $\left(u_{1}, u_{2}\right)^{\tau}=\left(u_{2}, u_{1}\right)$ for all $u_{1}, u_{2} \in V_{n}(p)$. Let $V_{1}:=\left\{(u, 0): u \in V_{n}(p)\right\}$ and $V_{2}:=\{(0, u)$ : $\left.u \in V_{n}(p)\right\}$. For each $i \in\{1,2\}$, let $\pi_{i}$ denote the projection of $G_{0, V_{1}}=G_{0} \cap\left(\operatorname{GL}_{n}(p) \times \operatorname{GL}_{n}(p)\right)$ onto the $i$-th factor of $\mathrm{GL}_{n}(p) \times \mathrm{GL}_{n}(p)$, and let $K_{i}$ and $G_{0}^{i}$ denote the kernel and image of $\pi_{i}$, respectively. The orbits of $G_{0}$ on $V^{*}$ are $V_{1}^{*} \cup V_{2}^{*}$ and $V_{n}(p)^{*} \times V_{n}(p)^{*}$. In particular, $G_{0}^{i}$ is transitive on $V_{n}(p)^{*}$ for both $i$, and $G_{0, V_{1}}$ is an index 2 subgroup of $G_{0}$. Now $G_{0}=G_{0, V_{1}}\langle(t, s) \tau\rangle$ for some $t, s \in \mathrm{GL}_{n}(p)$. Conjugating $G_{0}$ by $\left(s^{-1}, 1\right)$ if necessary, we may assume that $s=1$, in which case $G_{0}^{1}=G_{0}^{2}$ and $t \in G_{0}^{1}$ since $((t, 1) \tau)^{2}=(t, t)$. Now $G_{0} \leqslant G_{0}^{1} \imath\langle\tau\rangle$, and there exists $H \unlhd G_{0}^{1}$ such that $K_{1}=1 \times H$ and $K_{2}=H \times 1$. 
By Theorem 2.18, $G_{0}^{1}$ belongs to one of the classes (H0)-(H8). In particular, $G_{0}^{1} \leqslant \Gamma \mathrm{L}_{m}(q)$ where $q^{m}=p^{n}$, and either $G_{0}^{1}$ belongs to (H5)-(H8) and $q=p$, or $G_{0}^{1}$ belongs to (H0)-(H4) and $q$ is specified by Theorem 2.18. Now $G_{0} \leqslant \Gamma L_{m}(q) \imath\langle\tau\rangle$. Write $q=p^{e}$ and let $Z:=Z\left(\mathrm{GL}_{m}(q)\right)$.

If $G_{0}^{1}$ lies in class (H0) (i.e., if $m=1$ ), then $G_{0}$ lies in class (I0), so we assume that $m \geqslant 2$. If $G_{0}^{1}$ lies in (H7), then $G_{0}$ lies in (I7), and if $G_{0}^{1}$ lies in (H8), then $G_{0}$ lies in class (I8) by a computation in MAgma, so we may assume that $G_{0}^{1}$ lies in one of the classes (H1)-(H6).

Suppose for a contradiction that $H \leqslant Z$. Recall that $\left(q^{m}-1\right)^{2}$ divides $\left|G_{0}\right|=2\left|G_{0, V_{1}}\right|=$ $2\left|G_{0}^{1}\right||H|$. Hence $\left(q^{m}-1\right)^{2}$ divides $2\left|\Gamma \mathrm{L}_{m}(q)\right|(q-1)$. First suppose that $e m \geqslant 3$ and $(e m, p) \neq$ $(6,2)$. By Zsigmondy's theorem [58], there exists a primitive prime divisor $r$ of $p^{e m}-1$ (see [37, Theorem 5.2.14]). Let $f$ be the largest integer for which $r^{f}$ divides $q^{m}-1$. Now $r^{2 f}$ divides $\left(q^{m}-1\right)^{2}$, and $r$ is odd, so $r^{2 f}$ divides $\left|\Gamma \mathrm{L}_{m}(q)\right|(q-1)$, but $r$ is coprime to $q$ and does not divide $p^{i}-1$ for $i<e m$, so $r$ must divide $e$. However, $r \equiv 1 \bmod e m$ by [37, Proposition 5.2.15], a contradiction. Thus either $e m=2$ (so $m=2$ and $e=1$ ), or $(e m, p)=(6,2)$. Suppose that $m=2$. Now $\left(q^{2}-1\right)^{2}$ divides $2 q(q-1)^{2}\left(q^{2}-1\right) e$, so $q+1$ divides $2(q-1) e$. This is impossible when $p=2$ and $e=3$. Hence $e=1$. Now $p+1$ divides $2(p-1)$, but $(p+1, p-1)=(p-1,2)$, so $p=3$, in which case $G_{0}^{1}$ belongs to (H7), a contradiction. Further, if $q=4$ and $m=3$, then $\left(4^{3}-1\right)^{2}$ divides $6\left|\Gamma L_{3}(4)\right|$, a contradiction.

Thus $q=2$ and $m=6$. In particular, $H=1$, and $G_{0}^{1}$ is one of $G_{2}(2)^{\prime}=\operatorname{PSU}_{3}(3), G_{2}(2)=$ $\mathrm{PSU}_{3}(3): 2, \mathrm{Sp}_{6}(2)$ or $\mathrm{SL}_{6}(2)$. Since $\left(2^{6}-1\right)^{2}$ divides $2\left|G_{0}^{1}\right|$, we conclude that $G_{0}^{1}=\mathrm{SL}_{6}(2)$. Now there exists a bijective map $\alpha: \mathrm{SL}_{6}(2) \rightarrow \mathrm{SL}_{6}(2)$ such that $G_{0, V_{1}}=\left\{\left(g, g^{\alpha}\right): g \in \mathrm{SL}_{6}(2)\right\}$. Moreover, $\alpha$ is a homomorphism, so $\alpha \in \operatorname{Aut}\left(\mathrm{SL}_{6}(2)\right)=\mathrm{SL}_{6}(2):\langle\iota\rangle$ where $\iota$ maps each element of $\mathrm{SL}_{6}(2)$ to its inverse transpose. Since $G_{0, V_{1}}$ has index 2 in $G_{0}$ and $\left(2^{6}-1\right)^{2}$ is odd, it follows that $G_{0, V_{1}}$ is transitive on $V_{6}(2)^{*} \times V_{6}(2)^{*}$. Further, $G_{0, V_{1}}$ is conjugate in $\mathrm{SL}_{6}(2) \times \mathrm{SL}_{6}(2)$ to $\left\{(g, g): g \in \mathrm{SL}_{6}(2)\right\}$ or $\left\{\left(g, g^{\iota}\right): g \in \mathrm{SL}_{6}(2)\right\}$. However, neither of these is transitive, for if we view $V_{6}(2)$ as $\mathbb{F}_{2}^{6}$ and define $v_{1}:=(1,0,0,0,0,0)$ and $v_{2}:=(0,1,0,0,0,0)$, then $\left(v_{1}, v_{1}\right)$ and $\left(v_{1}, v_{2}\right)$ lie in different orbits.

Hence $H$ is a non-central normal subgroup of $G_{0}^{1}$. If $G_{0}^{1}$ lies in (H5) or (H6), then $H=G_{0}^{1}$, so $G_{0}$ lies in (I5) or (I6), respectively. Let $N$ be $\mathrm{SL}_{m}(q), \operatorname{Sp}_{m}(q), G_{2}(q)^{\prime}$ or $\mathrm{SL}_{2}(5)$ when $G_{0}^{1}$ lies in classes (H1)-(H4), respectively. Since $N Z / Z$ is the socle of the almost simple group $G_{0}^{1} Z / Z$, it follows that $N \unlhd H$. Hence $N \times N \unlhd G_{0}$, and $G_{0}$ lies in one of the classes (I1)-(I4).

Remark 3.4. Before we use [39] to prove Theorem 3.1, we discuss three minor oversights in the main theorem of [39]. This theorem states that if $G$ is an affine primitive permutation group of rank 3 with socle $V:=V_{d}(p)$ for some prime $p$, then $G_{0}$ belongs to one of 13 classes, and these classes are labelled (A1)-(A11), (B) and (C). The three issues are described below.

(1) In (A9), (A10) and (C), there is a non-abelian simple group $L$ such that $L \unlhd G_{0} / Z\left(G_{0}\right)$ where $L \leqslant \operatorname{PSL}_{a}(q)$ and $q^{a}=p^{d}$. Here $Z\left(G_{0}\right)$ should be replaced with $G_{0} \cap \mathbb{F}_{q}^{*}$. Indeed, except for the case $\left(L, p^{d}\right)=\left(A_{7}, 2^{8}\right)$ of $(\mathrm{C})$, such $G_{0}$ are analysed in [39] as part of [39, p.485, Case (I)], where the following hold: $G_{0} \leqslant \Gamma \mathrm{L}_{a}(q) ; L$ is the socle of $G_{0} /\left(G_{0} \cap \mathbb{F}_{q}^{*}\right)$; and the (projective) representation of $L$ on $V=V_{a}(q)$ is absolutely irreducible and cannot be realised over a proper subfield of $\mathbb{F}_{q}$. If $G_{0} \leqslant \mathrm{GL}_{a}(q)$, then $Z\left(G_{0}\right)=G_{0} \cap \mathbb{F}_{q}^{*}$ by [37, Lemma 2.10.1], but $G_{0} \cap \mathbb{F}_{q}^{*}$ is not a subgroup of $Z\left(G_{0}\right)$ in general.

(2) The case $\left(L, p^{d}\right)=\left(A_{7}, 2^{8}\right)$ of $(\mathrm{C})$ is different. Here $A_{7} \leqslant \mathrm{SL}_{4}(4)$ and $q=4$, but this representation of $A_{7}$ on $V_{4}(4)$ can be realised over $\mathbb{F}_{2}$ since $A_{7} \leqslant A_{8} \simeq \mathrm{SL}_{4}(2) \leqslant \mathrm{SL}_{4}(4)$. In $\left[39\right.$, p.483, Case (IIc)], Liebeck notes that $H_{0}:=\mathbb{F}_{4}^{*} \times A_{7}$ has two orbits on $V^{*}$, and since $H_{0} / \mathbb{F}_{4}^{*}$ is simple, $H_{0}$ lies in (C). Here Liebeck is classifying those $G$ for which $G_{0} \leqslant N_{\Gamma L_{4}(4)}\left(\mathrm{SL}_{4}(2)\right)$ and $G_{0} \cap \mathrm{SL}_{4}(2)=A_{7}$. Since $C_{\Gamma L_{4}(4)}\left(\mathrm{SL}_{4}(2)\right)=\mathbb{F}_{4}^{*}:\left\langle\sigma_{4}\right\rangle$, we may take $G_{0}$ to be $H_{0}\left\langle\sigma_{4}\right\rangle=\mathbb{F}_{4}^{*}:\left\langle\sigma_{4}\right\rangle \times A_{7}$, but the socle of $H_{0}\left\langle\sigma_{4}\right\rangle / \mathbb{F}_{4}^{*}$ is not simple, so $H_{0}\left\langle\sigma_{4}\right\rangle$ is not in (C). This is an omission of [39, p.483] but not [39] since $H_{0}\left\langle\sigma_{4}\right\rangle \simeq \Gamma L_{1}(4) \otimes A_{7}$, which stabilises a decomposition $V=V_{2}(2) \otimes V_{4}(2)$, so $H_{0}$ and $H_{0}\left\langle\sigma_{4}\right\rangle$ are in class (A3), 
and there are no other possibilities for $G_{0}$ by a computation in MAgma. Note that $H_{0}$ and $H_{0}\left\langle\sigma_{4}\right\rangle$ belong to class (T4) of Theorem 3.2, and they also belong to class (S2).

(3) When $L=A_{9}<\Omega_{8}^{+}(2)$, the subdegrees in [39, Table 14] are recorded as 105 and 150; these should be 120 and 135, which are, respectively, the numbers of non-singular and singular vectors with respect to the non-degenerate quadratic form preserved by $\Omega_{8}^{+}(2)$.

Proof of Theorem 3.1. By [39] and Remark 3.4, one of the following holds.

(1) (a) $G_{0} \leqslant \Gamma L_{1}\left(p^{d}\right)$.

(b) $V=V_{n}\left(q^{2}\right)$ and $\mathrm{SL}_{n}(q) \unlhd G_{0} \leqslant \Gamma L_{n}\left(q^{2}\right)$ where $q^{2 n}=p^{d}$ and $n \geqslant 2$ (see [39, p.482]).

(c) $V=V_{2}\left(q^{3}\right)$ and $\mathrm{SL}_{2}(q) \unlhd G_{0} \leqslant \Gamma L_{2}\left(q^{3}\right)$ where $q^{6}=p^{d}$ (see [39, p.483]).

(d) $V=\bigwedge^{2}\left(V_{5}(q)\right)$ and $\mathrm{SL}_{5}(q) \unlhd G_{0}$ where $q^{10}=p^{d}$.

(e) $\mathrm{Sz}(q)={ }^{2} B_{2}(q) \unlhd G_{0}$ where $q^{4}=p^{d}$ and $q$ is an odd power of 2 .

(2) $\mathrm{SU}_{n}(q) \unlhd G_{0}$ where $q^{2 n}=p^{d}$ and $n \geqslant 2$.

(3) $\Omega_{2 m}^{ \pm}(q) \unlhd G_{0}$ where $q^{2 m}=p^{d}$ and $m \geqslant 1$.

(4) $G_{0}$ stabilises a decomposition $V=V_{n}(p) \oplus V_{n}(p)$ where $2 n=d$.

(5) $G_{0}$ stabilises a decomposition $V=V_{2}(q) \otimes V_{m}(q)$ where $q^{2 m}=p^{d}$ and $m \geqslant 2$.

(6) $G_{0} \leqslant \Gamma L_{n}(q)$ and the socle $L$ of $G_{0} /\left(G_{0} \cap \mathbb{F}_{q}^{*}\right)$ is absolutely irreducible on $V=V_{n}(q)$ and cannot be realised over a proper subfield of $\mathbb{F}_{q}$. Further, one of the following holds.

(a) $V$ is a spin module, $L=\mathrm{P} \Omega_{m}^{\varepsilon}(q)$ and $(m, n, \varepsilon)$ is $(7,8, \circ)$ or $(10,16,+)$.

(b) $\left(L, p^{d}\right)$ and the embedding of $L$ in $\operatorname{PSL}_{n}(q)$ are given by [39, Table 2], where $\left(L, p^{d}\right) \neq$ $\left(A_{7}, 2^{8}\right)$. The subdegrees of $G$ are given by [25, Theorem 5.3] and [39, Table 14], except for $L=A_{9}$, in which case the subdegrees are 120 and 135 .

(7) $G_{0}$ normalises $E$ where $E \leqslant \mathrm{GL}_{d}(p)$ and $\left(E, p^{d}\right)$ is given by Table 5 (see $[37, \S 4.6]$ for details on extraspecial groups and [39, p.483, Case (IIe)]). The subdegrees of $G$ are given by [24, Theorem 1.1, 2(b) or 2( $\left.\left.\mathrm{b}^{\prime}\right)\right]$ when $E=D_{8}$ or $Q_{8}$, and [39, Table 13] otherwise.

\begin{tabular}{cc}
\hline$E$ & $p^{d}$ \\
\hline $3^{1+2}$ & $2^{6}$ \\
$D_{8}, Q_{8}$ & $3^{4}, 3^{6}, 7^{2}, 13^{2}, 17^{2}, 19^{2}, 23^{2}, 29^{2}, 31^{2}, 47^{2}$ \\
$Q_{8} \circ Q_{8}$ & $3^{4}$ \\
$D_{8} \circ Q_{8}$ & $3^{4}, 5^{4}, 7^{4}$ \\
$D_{8} \circ Q_{8} \circ\left\langle\zeta_{5}\right\rangle$ & $5^{4}$ \\
$Q_{8} \circ Q_{8} \circ Q_{8}$ & $3^{8}$ \\
\hline
\end{tabular}

TABLE 5.

We now consider each of the cases described above in order to prove that $G_{0}$ belongs to one of the classes in the statement of Theorem 3.1.

Case (1). If one of (a), (b), (c) or (d) holds, then $G_{0}$ lies in class (R0), (S1), (S0) or (R3), respectively. If (e) holds, then either $G_{0}$ lies in class (R4), or $q=2$ and $G_{0}$ lies in class (R0).

Case (2). Either $G_{0}$ lies in class (R1), or $n=2$, in which case $G_{0}$ lies in class (S1) since $\mathrm{SL}_{2}(q)$ and $\mathrm{SU}_{2}(q)$ are conjugate in $\mathrm{GL}_{d}(p)$. Indeed, let $\left\{x_{1}, x_{2}\right\}$ be a basis of $V_{2}\left(q^{2}\right)$ on which $\mathrm{SL}_{2}(q)$ acts naturally over $\mathbb{F}_{q}$, and choose $\mu \in \mathbb{F}_{q^{2}}^{*}$ such that $\mu+\mu^{q}=0$. Then $\operatorname{SL}_{2}(q)$ preserves the non-degenerate unitary form $\mathbf{f}$ for which $\mathbf{f}\left(x_{1}, x_{2}\right)=\mu$ and $\mathbf{f}\left(x_{i}, x_{i}\right)=0$ for $i=1,2$.

Case (3). Here one of the following holds: $G_{0}$ lies in class (R2); $m=2$ and $\varepsilon=+$, in which case $\Omega_{4}^{+}(q)$ and $\mathrm{SL}_{2}(q) \otimes \mathrm{SL}_{2}(q)$ are conjugate in $\mathrm{GL}_{d}(p)$, so $G_{0}$ lies in class (T1) for $q>2$ and class (I0) for $q=2$; or $m=1$, in which case $\varepsilon=+$ and $G_{0}$ lies in class (I0).

Case (4). By Theorem 3.3, $G_{0}$ lies in one of the classes (I0)-(I8).

Case (5). By Theorem 3.2, either $G_{0}$ belongs to one of the classes (R0), (I0), (T1), (T2) or (T3), in which case we are done, or $G_{0}$ lies in (T4), (T5) or (T6). If $G_{0}$ lies in class

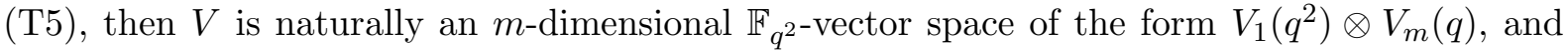


$\mathrm{SL}_{m}(q) \unlhd G_{0} \leqslant \Gamma \mathrm{L}_{m}\left(q^{2}\right)$, so $G_{0}$ belongs to class (S1). Similarly, if $G_{0}$ lies in class (T4), then since $\mathrm{GL}_{2}(2)=\Gamma \mathrm{L}_{1}\left(2^{2}\right)$, again $V$ is naturally a 4 -dimensional $\mathbb{F}_{2^{2}}$-vector space of the form $V_{1}\left(2^{2}\right) \otimes V_{4}(2)$, and $A_{7} \unlhd G_{0} \leqslant \Gamma L_{4}\left(2^{2}\right)$, so $G_{0}$ belongs to class (S2). Lastly, if $G_{0}$ lies in class (T6), then $V$ is naturally a 2-dimensional $\mathbb{F}_{q^{3}}$-vector space of the form $V_{2}(q) \otimes V_{1}\left(q^{3}\right)$, and $\mathrm{SL}_{2}(q) \unlhd G_{0} \leqslant \Gamma L_{2}\left(q^{3}\right)$, so $G_{0}$ belongs to class (S0).

Case (6). View $L$ as a subgroup of $G_{0} \mathbb{F}_{q}^{*} / \mathbb{F}_{q}^{*}$, and define $M$ to be the subgroup of $\operatorname{GL}_{n}(q)$ for which $M / \mathbb{F}_{q}^{*}=L$. Now $\mathbb{F}_{q}^{*}=Z(M)$, so by [1,31.1], $M=M^{\prime} \mathbb{F}_{q}^{*}$ and $M^{\prime}$ is quasisimple. Since $M \unlhd G_{0} \mathbb{F}_{q}^{*}$ and $M^{\prime}$ is perfect, it follows that $M^{\prime} \unlhd G_{0}$ and $M^{\prime} \leqslant \mathrm{SL}_{n}(q)$. If (a) holds, then $M^{\prime}=\operatorname{Spin}_{m}^{\varepsilon}(q)$ (see $\S 9$ for more details), so $G_{0}$ belongs to class (R5). Suppose instead that (b) holds. Using the $p$-modular character tables in [14, 26, 33], we determine that $M^{\prime}=S$, where $S$ is given by Table 3 . Now $S$ is absolutely irreducible on $V$ and cannot be realised over a proper subfield of $\mathbb{F}_{q}$, so $S$ is irreducible on $V_{d}(p)$ by [5, Theorems VII.1.16 and VII.1.17]. Thus $G_{0}$ belongs to class (AS).

Case (7). Where necessary, we use MAGMA to verify the various claims made below. If either $p^{d}=3^{8}$ and $E=Q_{8} \circ Q_{8} \circ Q_{8}$, or $p^{d}=7^{4}$ and $E=D_{8} \circ Q_{8}$, then $G_{0}$ belongs to (E). Suppose that $p^{d}=5^{4}$. If $E=D_{8} \circ Q_{8}$, then $G_{0}$ belongs to (E). Otherwise, $E=D_{8} \circ Q_{8} \circ\left\langle\zeta_{5}\right\rangle$, and we may assume that $G_{0} \nless N_{\mathrm{GL}_{4}(5)}\left(D_{8} \circ Q_{8}\right)$, in which case $G_{0}$ belongs to (E). Suppose that $p^{d}=2^{6}$ and $E$ is an extraspecial group $3^{1+2}$. Now $N_{\mathrm{GL}_{d}(p)}(E)$ is conjugate to $\Gamma \mathrm{U}_{3}(2)$, so we may assume that $N_{\mathrm{GL}_{d}(p)}(E)=\Gamma \mathrm{U}_{3}(2)$. If $\mathrm{SU}_{3}(2) \leqslant G_{0}$, then $G_{0}$ belongs to (R1); otherwise, $G_{0}$ belongs to (E).

Suppose that $d=2$, in which case $E=D_{8}$ or $Q_{8}$ and $p \in\{7,13,17,19,23,29,31,47\}$. For each such $p$, the group $\mathrm{GL}_{2}(p)$ contains a unique conjugacy class of subgroups isomorphic to $E$. If $p \neq 7$, then $N_{\mathrm{GL}_{2}(p)}\left(D_{8}\right)$ has at least 4 orbits on $V_{d}(p)$, so $E=Q_{8}$, in which case $G_{0}$ belongs to (E). Suppose instead that $p=7$. Now $N_{\mathrm{GL}_{2}(7)}\left(Q_{8}\right)$ is transitive on $V^{*}$ and contains, up to conjugacy in $\mathrm{GL}_{d}(p)$, five subgroups with two orbits on $V^{*}$; these have orders $24,24,24,36$ and 72. The group of order 36 has orbits of size 12 and 36 on $V^{*}$ and lies in (I0). The remaining four groups have two orbits of size 24 ; two of these are subgroups of $\Gamma L_{1}\left(7^{2}\right)$ and therefore belong to (R0), and the other two belong to (E). Lastly, if $E=D_{8}$, then $N_{\mathrm{GL}_{2}(7)}(E) \leqslant \Gamma L_{1}\left(7^{2}\right)$, so $G_{0}$ belongs to (R0).

Suppose that $p^{d}=3^{6}$ and $E=D_{8}$ or $Q_{8}$. Now $E \leqslant \Gamma L_{2}(27)$ by [24, Theorem 1.1]. The group $\Gamma \mathrm{L}_{2}(27)$ has a unique conjugacy class of subgroups isomorphic to $E$, and each such group is conjugate in $\mathrm{GL}_{6}(3)$ to $E \otimes 1 \leqslant \mathrm{GL}_{2}(3) \otimes \mathrm{GL}_{3}(3)$. The normalisers of $D_{8} \otimes 1$ and $Q_{8} \otimes 1$ in $\mathrm{GL}_{6}(3)$ are $\Gamma \mathrm{L}_{1}(9) \otimes \mathrm{GL}_{3}(3)$ and $\mathrm{GL}_{2}(3) \otimes \mathrm{GL}_{3}(3)$, respectively, so $G_{0}$ stabilises a decomposition $V=V_{2}(3) \otimes V_{3}(3)$, and we considered such groups in case (5) above.

Suppose that $p^{d}=3^{4}$ and $E=D_{8} \circ Q_{8}$. Now $N_{\mathrm{GL}_{4}(3)}(E)$ is transitive on $V^{*}$ and has, up to conjugacy in $\mathrm{GL}_{d}(p)$, exactly 18 subgroups with two orbits on $V^{*}$. Eight of these groups have orbit sizes 32 and 48; these lie in (S1). Another eight have orbit sizes 16 and 64; these stabilise a decomposition $V=V_{2}(3) \oplus V_{2}(3)$, and we considered such groups in case (4) above. This leaves two groups with orbit sizes 40 and 40 . One is a subgroup of $\Gamma L_{1}\left(3^{4}\right)$ and therefore lies in (R0). The other is isomorphic to $\mathrm{SL}_{2}(5)$ and lies in class (AS).

It remains to consider the case where $p^{d}=3^{4}$ and $E$ is one of $Q_{8}, D_{8}$ or $Q_{8} \circ Q_{8}$. If $E=D_{8}$ or $Q_{8}$, then $\mathrm{GL}_{4}(3)$ contains a unique conjugacy class of subgroups isomorphic to $E$ whose normalisers in $\mathrm{GL}_{4}(3)$ have 2 orbits on $V^{*}$, and this conjugacy class contains $E \otimes 1 \leqslant$ $\mathrm{GL}_{2}(3) \otimes \mathrm{GL}_{2}(3)$. The normalisers of $D_{8} \otimes 1$ and $Q_{8} \otimes 1$ in $\mathrm{GL}_{4}(3)$ are $\Gamma_{1}(9) \otimes \mathrm{GL}_{2}(3)$ and $\mathrm{GL}_{2}(3) \otimes \mathrm{GL}_{2}(3)$, respectively. Both of these normalisers are subgroups of $\left(\mathrm{GL}_{2}(3) \otimes \mathrm{GL}_{2}(3)\right): 2$, which is the normaliser in $\mathrm{GL}_{4}(3)$ of $Q_{8} \otimes Q_{8} \simeq Q_{8} \circ Q_{8}$, and $\mathrm{GL}_{4}(3)$ contains a unique conjugacy class of subgroups isomorphic to $Q_{8} \circ Q_{8}$. Thus, for each possible $E$, the group $G_{0}$ stabilises a decomposition $V=V_{2}(3) \otimes V_{2}(3)$, and we considered such groups in case (5) above.

In [39, Remark 3], Liebeck observes that if $G$ is an affine primitive permutation group of rank 3 on $V$, then $G_{0}$ has exactly two orbits on the set of one-dimensional subspaces of $V$ with 
one exception: those groups in class (AS) with $S=2 \cdot A_{5}$ and degree $3^{4}$. However, Liebeck does not specify the field over which this occurs, and the choice of field matters; indeed, in the exceptional case, $2 \cdot A_{5} \unlhd G_{0} \leqslant \Gamma L_{2}(9) \leqslant \mathrm{GL}_{4}(3)$, and $G_{0}$ is transitive on the one-dimensional subspaces of $V_{2}(9)$ but has two orbits on the set of one-dimensional subspaces of $V_{4}(3)$. In the following, we give an explicit statement of [39, Remark 3], which we deduce from [39].

Corollary 3.5. Let $G$ be an affine primitive permutation group of rank 3 on $V_{d}(p)$ where $d \geqslant 1$ and $p$ is prime, and let $s^{c}=p^{d}$ where one of the following holds (in the notation of Theorem 3.1).

(i) $G_{0}$ lies in class $\mathcal{C}$, where $\mathcal{C}$ and $(c, s)$ are given by Table 6.

(ii) $G_{0}$ lies in class (E) and either $(c, s)=(3,4)$, or $p$ is odd and $(c, s)=(d, p)$.

(iii) $G_{0}$ lies in class $(\mathrm{AS})$, and $(c, s)=(d, p)$ unless $\left(S, p^{d}\right)$ is one of $\left(2 \cdot A_{5}, 3^{4}\right),\left(2 \cdot A_{5}, 7^{4}\right)$, $\left(3 \cdot A_{6}, 2^{6}\right)$ or $\left(J_{2}, 2^{12}\right)$, in which case $(c, s)=\left(d / 2, p^{2}\right)$.

Then $G_{0} \leqslant \Gamma L_{c}(s)$. Further, $G_{0}$ has two orbits on the points of $\mathrm{PG}_{c-1}(s)$ unless $G_{0}$ lies in $(\mathrm{R} 0)$ or (AS) with $\left(S, p^{d}\right)=\left(2 \cdot A_{5}, 3^{4}\right)$, in which case $G_{0}$ is transitive on the points of $\mathrm{PG}_{c-1}(s)$.

\begin{tabular}{|c|c|c|c|c|c|c|c|c|c|c|}
\hline $\begin{array}{c}\mathcal{C} \\
(c, s)\end{array}$ & $\begin{array}{c}\mathrm{R} 0) \\
\left(1, p^{d}\right)\end{array}$ & $\begin{array}{c}(\mathrm{R} 1) \\
\left(n, q^{2}\right)\end{array}$ & $\begin{array}{c}(\mathrm{R} 2) \\
(2 m, q)\end{array}$ & $\begin{array}{c}\text { (R3) } \\
(10, q)\end{array}$ & $\begin{array}{l}\mathrm{R} 4) \\
(4, q)\end{array}$ & $\begin{array}{l}\mathrm{R} 5) \\
(n, q)\end{array}$ & $\begin{array}{c}\mathrm{T} 1)-(\mathrm{T} 3) \\
(2 m, q)\end{array}$ & $\begin{array}{c}(\mathrm{S} 0) \\
\left(2, q^{3}\right)\end{array}$ & $\begin{array}{c}(\mathrm{S} 1)-(\mathrm{S} 2) \\
\left(n, q^{2}\right)\end{array}$ & $\begin{array}{c}(\mathrm{I}) \\
(d, p)\end{array}$ \\
\hline
\end{tabular}

We now use the pair $(c, s)$ from Corollary 3.5 to state Hypothesis 3.6, which was alluded to in Example 1.1 and Theorem 1.4.

Hypothesis 3.6. Let $(G, a, r)$ be defined as follows: $G$ is an affine primitive permutation group of rank 3 on $V:=V_{d}(p)$ where $d \geqslant 2$ and $p$ is prime, $r^{a}=p^{d}$ and one of the following holds, where $s^{c}=p^{d}$ and $(c, s)$ is given by Corollary 3.5.

(i) $G_{0}$ lies in one of (R1)-(R5), (T1)-(T3), (S0)-(S2) or (E), and $\mathbb{F}_{r}$ is a subfield of $\mathbb{F}_{s}$.

(ii) $G_{0}$ lies in $(\mathrm{AS})$, and $\mathbb{F}_{r}$ is a subfield of $\mathbb{F}_{s}$ unless $\left(S, p^{d}\right)=\left(2 \cdot A_{5}, 3^{4}\right)$, in which case $r=p$.

(iii) $V=V_{b}(r) \oplus V_{b}(r)$ and $G_{0} \leqslant\left(\Gamma L_{b}(r) \imath S_{2}\right) \cap \Gamma L_{2 b}(r)$ where $a=2 b$.

Corollary 3.7. If $(G, a, r)$ satisfies Hypothesis 3.6, then $G_{0} \leqslant \Gamma \mathrm{L}_{a}(r)$ and $G_{0}$ has two orbits on the points of $\mathrm{PG}_{a-1}(r)$.

Proof. Suppose that $(G, a, r)$ satisfies Hypothesis 3.6, and let $V:=V_{d}(p)$ where $p^{d}=r^{a}$ and $p$ is prime. Now $G_{0}$ has two orbits on $V^{*}$. In particular, if $G_{0} \leqslant \Gamma L_{m}(q)$ where $q^{m}=p^{d}$, then $G_{0}$ has two orbits on the points of $\mathrm{PG}_{m-1}(q)$ if and only if $\langle u\rangle_{\mathbb{F}_{q}}^{*} \subseteq u^{G_{0}}$ for all $u \in V^{*}$. Note also that if $t^{n}=q^{m}$ where $\mathbb{F}_{t}$ is a subfield of $\mathbb{F}_{q}$, then $\Gamma \mathrm{L}_{m}(q) \leqslant \Gamma \mathrm{L}_{n}(t)$. If $V=V_{b}(r) \oplus V_{b}(r)$ and $G_{0} \leqslant\left(\Gamma \mathrm{L}_{b}(r)<S_{2}\right) \cap \Gamma \mathrm{L}_{2 b}(r)$ where $a=2 b$, then clearly $G_{0} \leqslant \Gamma \mathrm{L}_{a}(r)$ and $G_{0}$ has two orbits on the points of $\mathrm{PG}_{a-1}(r)$. If $G_{0}$ lies in one of (R1)-(R5), (T1)-(T3), (S0)-(S2), (E) or (AS) with $\left(S, p^{d}\right) \neq\left(2 \cdot A_{5}, 3^{4}\right)$, and if $s^{c}=p^{d}$ where $(c, s)$ is given by Corollary 3.5, then by Corollary 3.5, $G_{0} \leqslant \Gamma \mathrm{L}_{c}(s)$ and $G_{0}$ has two orbits on the points of $\mathrm{PG}_{c-1}(s)$, so if $\mathbb{F}_{r}$ is a subfield of $\mathbb{F}_{s}$, then $G_{0} \leqslant \Gamma \mathrm{L}_{a}(r)$ and $G_{0}$ has two orbits on the points of $\mathrm{PG}_{a-1}(r)$. Lastly, suppose that $G_{0}$ lies in class (AS) with $\left(S, p^{d}\right)=\left(2 \cdot A_{5}, 3^{4}\right)$ and $r=p$. Clearly $G_{0} \leqslant \mathrm{GL}_{4}(3)$, and $-1 \in G_{0}$ since $2 \cdot A_{5} \unlhd G_{0}$ and $2 \cdot A_{5}$ is irreducible on $V$, so $\{u,-u\} \subseteq u^{G_{0}}$ for all $u \in V^{*}$. Thus $G_{0}$ has two orbits on the points of $\mathrm{PG}_{3}(3)$.

Remark 3.8. Let $V:=V_{b}(r) \oplus V_{b}(r)$ and $p^{d}=r^{2 b}$ where $p$ is prime, $r$ is not prime and $b \geqslant 1$. Let $G:=\mathrm{A} \mathrm{L}_{b}(r) \imath S_{2}$. Then $G_{0}=\Gamma \mathrm{L}_{b}(r) \imath S_{2}$, and $G$ is an affine primitive permutation group of rank 3 on $V_{d}(p)$ by Lemma 2.2. However, we claim that $(G, 2 b, r)$ does not satisfy Hypothesis 3.6. Suppose for a contradiction that it does. Now (iii) does not hold since $G_{0}$ is not a subgroup of $\Gamma L_{2 b}(r)$ (see $\S 13$ for more details), so (i) or (ii) holds. Recall from Table 4 that $G$ has subdegrees $2\left(r^{b}-1\right)$ and $\left(r^{b}-1\right)^{2}$. If (ii) holds, then $G_{0}$ lies in class (AS) and $r=s=p^{2}$, so $\left(S, p^{d}\right)$ is $\left(2 \cdot A_{5}, 7^{4}\right)$ or $\left(J_{2}, 2^{12}\right)$, but this is impossible by the subdegrees listed in Table 3 . Thus (i) holds. 
If $G_{0}$ lies in class (E), then $r=s=4$ and $p^{d}=2^{6}$, a contradiction. Thus $G_{0}$ lies in one of (R1)-(R5), (T1)-(T3) or (S0)-(S2). By Table 4, there is a subdegree $t$ of $G$ that is divisible by $p$. Then $t=2\left(r^{b}-1\right), p=2$, and 2 is the highest power of $p$ that divides $t$. Since $r \leqslant s$ and $r$ is not prime, it follows that $G_{0}$ lies in class (S1) with $(n, q)=(2,2)$ and $(b, r)=(1,4)$, but then $\mathrm{SL}_{2}(2) \unlhd G_{0}=\mathrm{SL}_{2}(2)$ \ $S_{2}$, a contradiction.

\section{Dependent partial Linear SPaCes}

Let $V:=V_{n}(q)$ where $q$ is a prime power and $n \geqslant 1$. A partial linear space $\mathcal{S}:=(V, \mathcal{L})$ is $\mathbb{F}_{q}$-dependent if $L \subseteq\langle x\rangle_{\mathbb{F}_{q}}$ for every $L \in \mathcal{L}_{0}$ and $x \in L^{*}$, and $\mathbb{F}_{q}$-independent otherwise. Observe that if $\mathcal{S}$ is $\mathbb{F}_{q}$-dependent, then it is $\mathbb{F}_{q^{m}}$-dependent for all divisors $m$ of $n$ since we may view $V$ as a vector space $V_{n / m}\left(q^{m}\right)$. In other words, if $\mathcal{S}$ is $\mathbb{F}_{q}$-independent, then it is $\mathbb{F}_{r}$-independent for all subfields $\mathbb{F}_{r}$ of $\mathbb{F}_{q}$.

Using Kantor's classification [36, Proposition 4.1] of the $G$-affine linear spaces for which $G$ is 2 -transitive and $G \leqslant \mathrm{~A}_{1}(q)$ (see also Theorem 2.19), we prove the following.

Lemma 4.1. Let $V:=V_{n}(q)$ where $n \geqslant 1$ and $q$ is a prime power. Let $(V, \mathcal{L})$ be a $G$-affine partial linear space where $G_{0} \leqslant \Gamma L_{n}(q)$. Let $L \in \mathcal{L}_{0}$ and $u \in L^{*}$. If $\langle u\rangle^{*} \subseteq u^{G_{0}}$, then $\left\{\lambda \in \mathbb{F}_{q}: \lambda u \in L\right\}$ is either $\{0,1\}$ or a subfield of $\mathbb{F}_{q}$.

Proof. Let $\mathcal{S}:=(V, \mathcal{L})$. Let $U:=\langle u\rangle$ and $H:=U: G_{0, U}^{U} \leqslant \mathrm{A \Gamma L}_{1}(q)$. By Lemma 2.13, $\mathcal{S} \cap U$ is a partial linear space, and $H \leqslant \operatorname{Aut}(\mathcal{S} \cap U)$. If $\lambda u, \mu u \in U^{*}$, then by assumption there exists $g \in G_{0}$ such that $(\lambda u)^{g}=\mu u$, so $U^{g}=U$. Thus $H$ acts 2-transitively on $U$. Let $F:=\left\{\lambda \in \mathbb{F}_{q}: \lambda u \in L\right\}$ and $k:=|F| \geqslant 2$. If $k=2$, then $F=\{0,1\}$, so we may assume that $k \geqslant 3$. Now $\mathcal{S} \cap U$ is an $H$-affine linear space whose line set contains $L \cap U$, and $H$ lies in class (H0), so Theorem 2.19(i) or (ii) holds. In either case, $F$ is a subfield of $\mathbb{F}_{q}$.

Let $V:=V_{n}(q)$ where $n \geqslant 2$ and $q$ is a prime power such that $q>2$. Let $G$ be an affine permutation group of rank 3 on $V$, so that $G_{0} \leqslant \Gamma L_{n}(q)$ (see $\left.\S 2.5\right)$. Observe that the following three statements are equivalent.

(i) $G_{0}$ has two orbits on the points of $\mathrm{PG}_{n-1}(q)$.

(ii) $\langle u\rangle^{*} \subseteq u^{G_{0}}$ for all $u \in V^{*}$.

(iii) $\langle x\rangle^{*} \subseteq x^{G_{0}}$ for some $x \in V^{*}$.

Now suppose that $G_{0}$ has two orbits $\Sigma_{1}$ and $\Sigma_{2}$ on the points of $\mathrm{PG}_{n-1}(q)$, and let $\left\langle x_{1}\right\rangle \in \Sigma_{1}$ and $\left\langle x_{2}\right\rangle \in \Sigma_{2}$. Let $X_{1}$ and $X_{2}$ be the orbits of $G_{0}$ on $V^{*}$ containing $x_{1}$ and $x_{2}$, respectively, and note that $V^{*}=X_{1} \cup X_{2}$. In Example 1.1 (for $G$ primitive), we constructed two $G$-affine proper partial linear spaces $\mathcal{S}_{i}:=\left(V, \mathcal{L}_{i}\right)$, where $\mathcal{L}_{i}:=\left\{\langle u\rangle+v:\langle u\rangle \in \Sigma_{i}, v \in V\right\}=\left\{\langle u\rangle+v: u \in X_{i}, v \in V\right\}$ for $i \in\{1,2\}$. Observe that $\mathcal{S}_{1}$ and $\mathcal{S}_{2}$ are $\mathbb{F}_{q}$-dependent partial linear spaces. Let $F:=\mathbb{F}_{r}$ be a subfield of $\mathbb{F}_{q}$ with $r>2$ where $r^{a}=q^{n}$. Now $G_{0} \leqslant \Gamma L_{n}(q) \leqslant \Gamma L_{a}(r)$, and $G_{0}$ has two orbits $\Delta_{1}$ and $\Delta_{2}$ on the points of $\mathrm{PG}_{a-1}(r)$, where $\left\langle x_{1}\right\rangle_{F} \in \Delta_{1}$ and $\left\langle x_{2}\right\rangle_{F} \in \Delta_{2}$. Thus both $\left(V,\left\{\langle u\rangle_{F}+v: u \in X_{1}, v \in V\right\}\right)$ and $\left(V,\left\{\langle u\rangle_{F}+v: u \in X_{2}, v \in V\right\}\right)$ are $\mathbb{F}_{q}$-dependent $G$-affine proper partial linear spaces from Example 1.1 (when $G$ is primitive). Using Lemma 4.1, we now prove that every $\mathbb{F}_{q}$-dependent $G$-affine proper partial linear space has this form for some subfield $F$.

Proposition 4.2. Let $V:=V_{n}(q)$ where $n \geqslant 2$ and $q$ is a prime power, and let $G$ be an affine permutation group of rank 3 on $V$ where $\langle x\rangle^{*} \subseteq x^{G_{0}}$ for some $x \in V^{*}$. Let $(V, \mathcal{L})$ be an $\mathbb{F}_{q^{-}}$ dependent $G$-affine proper partial linear space. Then there exists a subfield $F$ of $\mathbb{F}_{q}$ with $|F|>2$ and an orbit $X$ of $G_{0}$ on $V^{*}$ such that $\mathcal{L}=\left\{\langle u\rangle_{F}+v: u \in X, v \in V\right\}$.

Proof. By assumption, $G_{0} \leqslant \Gamma L_{n}(q)$. There exists $L \in \mathcal{L}_{0}$ and $w \in L^{*}$. Since $\langle x\rangle^{*} \subseteq x^{G_{0}}$, it follows that $\langle w\rangle^{*} \subseteq w^{G_{0}}$. By assumption, $L \subseteq\langle w\rangle$ and $|L| \geqslant 3$, so by Lemma 4.1, there exists a subfield $F$ of $\mathbb{F}_{q}$ with $|F|>2$ such that $L=\langle x\rangle_{F}$. Hence $\mathcal{L}=\left\{\langle u\rangle_{F}+v: u \in w^{G_{0}}, v \in V\right\}$. 
Let $G$ be an affine primitive permutation group of rank 3 on $V:=V_{d}(p)$ where $d \geqslant 1$ and $p$ is prime. If $G_{0}$ belongs to one of (R1)-(R5), (T1)-(T3) or (S0)-(S2), then by Corollary 3.5, $V=V_{c}(s)$ and $G_{0} \leqslant \Gamma \mathrm{L}_{c}(s)$ where $(c, s)$ is given by Table 6 , and $\langle x\rangle_{\mathbb{F}_{s}}^{*} \subseteq x^{G_{0}}$ for all $x \in V^{*}$, so each $\mathbb{F}_{s}$-dependent $G$-affine proper partial linear space is given by Proposition 4.2 and therefore Example 1.1 with respect to some triple $\left(G, \log _{r}\left(p^{d}\right), r\right)$ that satisfies Hypothesis 3.6. Thus for those $G_{0}$ in classes (R1)-(R5), (T1)-(T3) and (S0)-(S2), it remains to consider $\mathbb{F}_{s}$-independent $G$-affine proper partial linear spaces. This we do in $\S 5-12$, and we will see that Lemma 2.15 imposes severe restrictions on the possible examples; in particular, for $G_{0}$ in classes (R1)-(R5), there is a unique $\mathbb{F}_{s}$-independent $G$-affine proper partial linear space, and this example arises in class (R2) with $G=3^{4}: M_{10} \simeq 3^{4}:\left(\Omega_{4}^{-}(3) .2\right)$. However, for $G_{0}$ in classes (I0)-(I8), since $G_{0}$ need not be a subgroup of $\Gamma L_{2 m}(q)$, it is no longer convenient to make the distinction between $\mathbb{F}_{q}$-dependent and $\mathbb{F}_{q}$-independent $G$-affine proper partial linear spaces in our proofs; instead, in $\S 13$, we develop methods for building such partial linear spaces from 2-transitive affine linear spaces. Similarly, for $G_{0}$ in classes (E) and (AS), our methods are primarily computational (see $\S 14$ and $\S 15)$, so it is again more convenient not to make the distinction between dependent and independent $G$-affine proper partial linear spaces.

The next two results are also consequences of Lemma 4.1. When $q^{n}=p^{d}$ for a prime $p$, we say that a subset $L$ of $V:=V_{n}(q)$ is an affine $\mathbb{F}_{p}$-subspace of $V$ if $L$ is an affine subspace of the $\mathbb{F}_{p}$-vector space $V$.

Lemma 4.3. Let $V:=V_{n}(q)$ where $n \geqslant 1$ and $q$ is a power of a prime $p$, and let $G$ be an affine permutation group of rank 3 on $V$ where $\langle x\rangle^{*} \subseteq x^{G_{0}}$ for some $x \in V^{*}$. Let $\mathcal{S}:=(V, \mathcal{L})$ be a $G$-affine proper partial linear space whose lines are affine $\mathbb{F}_{p}$-subspaces of $V$. Then there exists a subfield $F$ of $\mathbb{F}_{q}$ such that each $L \in \mathcal{L}_{0}$ is an $F$-subspace of $V$ with the property that $F=\left\{\lambda \in \mathbb{F}_{q}: \lambda u \in L\right\}$ for all $u \in L^{*}$.

Proof. Let $L \in \mathcal{L}_{0}$ and $B:=L^{*}$. By Lemma 2.6, $B$ is a block of $G_{0}$ on $\mathcal{S}(0)$. For $u \in B$, let $F_{u}:=\left\{\lambda \in \mathbb{F}_{q}: \lambda u \in L\right\}$. First we claim that $F_{u}$ is a subfield of $\mathbb{F}_{q}$ for all $u \in B$. Let $u \in B$, and note that $\{0,1\} \subseteq F_{u}$. If $\left|F_{u}\right| \geqslant 3$, then the claim holds by Lemma 4.1. Otherwise, $\left|F_{u}\right|=2$, but $\left|F_{u}\right| \geqslant p$ since $L$ is an $\mathbb{F}_{p^{-}}$subspace of $V$, so $p=2$, and the claim follows. Let $u, v \in B$. Now $v=u^{g}$ for some $g \in G_{0}$, so $B=B^{g}$, and $g$ is $\sigma$-semilinear for some $\sigma \in \operatorname{Aut}\left(\mathbb{F}_{q}\right)$, so $F_{v}=F_{u}^{\sigma}=F_{u}$. Thus we may define $F:=F_{u}$ for $u \in B$, and $L$ is an $F$-subspace of $V$. If $L^{\prime} \in \mathcal{L}_{0}$, then $L^{\prime}=L^{g}$ for some $g \in G_{0}$, so $L^{\prime}$ also has the desired structure.

Lemma 4.4. Let $V:=V_{n}(q)$ where $n \geqslant 1$ and $q$ is a power of a prime $p$, and let $G$ be an affine permutation group of rank 3 on $V$ where $\langle x\rangle^{*} \subseteq x^{G_{0}}$ for some $x \in V^{*}$. Let $\mathcal{S}:=(V, \mathcal{L})$ be a $G$-affine proper partial linear space. If $|L \cap\langle u\rangle| \geqslant 3$ for some $L \in \mathcal{L}_{0}$ and $u \in L^{*}$, then every line of $\mathcal{S}$ is an affine $\mathbb{F}_{p}$-subspace of $V$.

Proof. If $|L \cap\langle u\rangle| \geqslant 3$ for some $L \in \mathcal{L}_{0}$ and $u \in L^{*}$, then $\left\{\lambda \in \mathbb{F}_{q}: \lambda u \in L\right\}$ is a subfield of $\mathbb{F}_{q}$ by Lemma 4.1, so $(L \cap\langle u\rangle)^{\tau_{u}}=L \cap\langle u\rangle$. Thus $L^{\tau_{u}}=L$, and we may apply Lemma 2.16.

Now we construct some dependent $G$-affine proper partial linear spaces for $G$ in class (R0).

Example 4.5. Let $q:=p^{d}$ and $V:=\mathbb{F}_{q}$ where $p$ is prime and $d \geqslant 2$. Let $G$ be an affine primitive permutation group of rank 3 on $V$, so that $G_{0} \leqslant \Gamma L_{1}(q)$. Let $F:=\mathbb{F}_{r}$ be a proper subfield of $\mathbb{F}_{q}$ with $r>2$ such that $\langle x\rangle_{F}^{*} \subseteq x^{G_{0}}$ for $x \in V^{*}$. Write $q=r^{a}$. Then $G_{0} \leqslant \Gamma \mathrm{L}_{a}(r)$, and $G_{0}$ has two orbits on the points of $\mathrm{PG}_{a-1}(r)$, so $\left(V,\left\{\langle u\rangle_{F}+v: u \in x^{G_{0}}, v \in V\right\}\right)$ is a $G$-affine proper partial linear space from Example 1.1 for $x \in V^{*}$.

To see that such $G$ and $F$ exist, assume that $p$ is odd and $d$ is even, and choose $F$ to be any subfield of $V=\mathbb{F}_{q}$ for which $\left[\mathbb{F}_{q}: F\right]$ is even. Let $\zeta:=\zeta_{q}$ and $\sigma:=\sigma_{q}$ (see $\S 2.3$ ). Then $\Gamma L_{1}(q)=\langle\zeta, \sigma\rangle$, and the orbits of $\left\langle\zeta^{2}, \sigma\right\rangle$ on $V^{*}$ are the squares $\left\langle\zeta^{2}\right\rangle$ and the non-squares $\left\langle\zeta^{2}\right\rangle \zeta$ of $\mathbb{F}_{q}^{*}$. Let $G:=V: G_{0}$, where $G_{0}$ is any subgroup of $\left\langle\zeta^{2}, \sigma\right\rangle$ with two orbits on $V^{*}$. Then $G$ is an affine permutation group of rank 3 on $V$, and $G$ is primitive by Lemma 2.2 since 
$\left|x^{G_{0}} \cup\{0\}\right|=(q+1) / 2$ for $x \in V^{*}$. Further, since $\left[\mathbb{F}_{q}: F\right]$ is even, every element of $\langle 1\rangle_{F}^{*}=F^{*}$ is a square in $\mathbb{F}_{q}^{*}$, so $\langle x\rangle_{F}^{*} \subseteq x^{G_{0}}$ for $x \in V^{*}$. Thus $\mathcal{S}:=\left(V,\left\{\langle u\rangle_{F}+v: u \in\left\langle\zeta^{2}\right\rangle, v \in V\right\}\right)$ is a $G$-affine proper partial linear space from Example 1.1. In fact, $\operatorname{Aut}(\mathcal{S})=V:\left\langle\zeta^{2}, \sigma\right\rangle$ since $V:\left\langle\zeta^{2}, \sigma\right\rangle$ is the automorphism group of the Paley graph with vertex set $\mathbb{F}_{q}$, in which vertices $x$ and $y$ are adjacent if and only if $x-y$ is a square in $\mathbb{F}_{q}^{*}$ (see [34, Theorem 9.1]).

\section{Class (R1)}

Let $G$ be an affine permutation group of rank 3 on $V:=V_{n}\left(q^{2}\right)$ for which $\operatorname{SU}_{n}(q) \unlhd G_{0}$ where $n \geqslant 3$. The orbits of $G_{0}$ on $V^{*}$ are the non-zero isotropic vectors and the non-isotropic vectors. Note that $\mathrm{SU}_{n}(q)$ acts transitively on the set of non-zero isotropic vectors for $n \geqslant 3$ by [37, Lemma 2.10.5]. In order to simplify the proofs in this section, we define a standard basis for a unitary space. Let $\mathbf{f}$ be the non-degenerate unitary form preserved by $\operatorname{SU}_{n}(q)$, and let $m$ be such that $n=2 m$ or $n=2 m+1$. By [37, Proposition 2.3.2], $V$ has a standard basis $\left\{e_{1}, \ldots, e_{m}, f_{1}, \ldots, f_{m}\right\}$ or $\left\{e_{1}, \ldots, e_{m}, f_{1}, \ldots, f_{m}, x_{0}\right\}$ when $n=2 m$ or $n=2 m+1$, respectively, where $\mathbf{f}\left(e_{i}, e_{j}\right)=\mathbf{f}\left(f_{i}, f_{j}\right)=0$ and $\mathbf{f}\left(e_{i}, f_{j}\right)=\delta_{i, j}$ for all $i, j$, and when $n$ is odd, $\mathbf{f}\left(x_{0}, x_{0}\right)=1$ and $\mathbf{f}\left(e_{i}, x_{0}\right)=\mathbf{f}\left(f_{i}, x_{0}\right)=0$ for all $i$.

Proposition 5.1. Let $V:=V_{n}\left(q^{2}\right)$ where $n \geqslant 3$ and $q$ is a prime power, and let $G$ be an affine permutation group of rank 3 on $V$ where $\mathrm{SU}_{n}(q) \unlhd G_{0}$. Then there is no $\mathbb{F}_{q^{2}}$-independent $G$-affine proper partial linear space.

Proof. Let $F:=\mathbb{F}_{q^{2}}$ and $\bar{\lambda}:=\lambda^{q}$ for $\lambda \in F$. Let $T: F \rightarrow \mathbb{F}_{q}$ be the trace map $\lambda \mapsto \lambda+\bar{\lambda}$, and let $N: F \rightarrow \mathbb{F}_{q}$ be the norm map $\lambda \mapsto \lambda \bar{\lambda}$. Note that both $T$ and $N$ are surjective maps. Further, $T$ has kernel $K:=\mathbb{F}_{q} \tau$ for some $\tau \in F^{*}$.

Suppose for a contradiction that $(V, \mathcal{L})$ is an $F$-independent $G$-affine proper partial linear space. Let $\mathbf{f}$ be the non-degenerate unitary form preserved by $\mathrm{SU}_{n}(q)$. Let $L \in \mathcal{L}_{0}$, let $B:=L^{*}$, and let $x \in L^{*}$. By Lemma 2.6, $B$ is a block of $G_{0}$ on $x^{G_{0}}$. Since $(V, \mathcal{L})$ is $F$-independent, $B \backslash\langle x\rangle$ is non-empty. Let $y \in B \backslash\langle x\rangle$.

First suppose that $x$ is isotropic. Let $m$ be such that $n=2 m$ or $n=2 m+1$. Let $\left\{e_{1}, \ldots, e_{m}, f_{1}, \ldots, f_{m}\right\}$ or $\left\{e_{1}, \ldots, e_{m}, f_{1}, \ldots, f_{m}, x_{0}\right\}$ be a standard basis of $V$ when $n=2 m$ or $n=2 m+1$, respectively. In either case, we denote this basis by $\mathcal{B}$. We may assume without loss of generality that $x=e_{1}$. For $\lambda \in F$, define $V_{x, \lambda}:=\left\{w \in V^{*}: \mathbf{f}(w, w)=0\right.$ and $\left.\mathbf{f}(x, w)=\lambda\right\}$.

We claim that if $\lambda \neq 0$, then $\mathrm{SU}_{n}(q)_{x}$ acts transitively on $V_{x, \lambda}$. We may assume that $\lambda=1$. Note that $f_{1} \in V_{x, 1}$, and let $u \in V_{x, 1}$. Then $u=\varepsilon e_{1}+f_{1}+u_{0}$ for some $\varepsilon \in F$ and $u_{0} \in\left\langle e_{1}, f_{1}\right\rangle^{\perp}$. There exists $g \in \mathrm{SL}_{n}\left(q^{2}\right)$ such that $e_{1}^{g}=e_{1}, f_{1}^{g}=u$ and $w^{g}=-\mathbf{f}(w, u) e_{1}+w$ for $w \in \mathcal{B} \backslash\left\{e_{1}, f_{1}\right\}$. Now $\mathbf{f}\left(v_{1}^{g}, v_{2}^{g}\right)=\mathbf{f}\left(v_{1}, v_{2}\right)$ for all $v_{1}, v_{2} \in V$, so $g \in \mathrm{SU}_{n}(q)_{x}$ and the claim holds.

Next we claim that if $n \geqslant 5$, then $\mathrm{SU}_{n}(q)_{x}$ acts transitively on $V_{x, 0} \backslash\langle x\rangle$. Note that $e_{2} \in$ $V_{x, 0} \backslash\langle x\rangle$, and let $u \in V_{x, 0} \backslash\langle x\rangle$. Then $u=\varepsilon e_{1}+u_{0}$ for some $\varepsilon \in F$ and non-zero isotropic vector $u_{0} \in\left\langle e_{1}, f_{1}\right\rangle^{\perp}$. Since $n \geqslant 4$, there exists $g \in \mathrm{SL}_{n}\left(q^{2}\right)$ such that $e_{2}^{g}=\varepsilon e_{1}+e_{2}, f_{1}^{g}=f_{1}-\bar{\varepsilon} f_{2}$ and $w^{g}=w$ for $w \in \mathcal{B} \backslash\left\{e_{2}, f_{1}\right\}$. Now $\mathbf{f}\left(v_{1}^{g}, v_{2}^{g}\right)=\mathbf{f}\left(v_{1}, v_{2}\right)$ for all $v_{1}, v_{2} \in V$, so $g \in \operatorname{SU}_{n}(q)_{x}$. Moreover, we may view $\mathrm{SU}_{n-2}(q)$ as a subgroup of $\mathrm{SU}_{n}(q)$ that fixes $\left\langle e_{1}, f_{1}\right\rangle$ pointwise and acts naturally on $\left\langle e_{1}, f_{1}\right\rangle^{\perp}$, and there exists $h \in \mathrm{SU}_{n-2}(q)$ such that $e_{2}^{h}=u_{0}$ by [37, Lemma 2.10.5] since $n \geqslant 5$. Now $g h \in \mathrm{SU}_{n}(q)_{x}$ and $e_{2}^{g h}=u$, so the claim holds.

Choose $\mu \in F \backslash K$ and let $\lambda:=\mathbf{f}(x, y)$. First suppose that either $n \geqslant 4$ and $\lambda \neq 0$, or $n \geqslant 5$ and $\lambda=0$. Now $\mathrm{SU}_{n}(q)_{x}$ acts transitively on $V_{x, \lambda} \backslash\langle x\rangle$ by the claims above, so $V_{x, \lambda} \backslash\langle x\rangle \subseteq B$ by Lemma 2.15(ii). In particular, $\bar{\lambda} f_{1}+\mu e_{2}$ and $\bar{\lambda} f_{1}+f_{2}$ are elements of $B$, but then $z:=\mu e_{2}-f_{2}$ is isotropic by Lemma 2.15(i), a contradiction since $\mathbf{f}(z, z)=-(\mu+\bar{\mu}) \neq 0$.

Next suppose that $n=3$. Now $V_{x, 0}=\langle x\rangle$, so $\lambda \neq 0$. Again, $V_{x, \lambda} \subseteq B$ by Lemma 2.15(ii). Choose distinct $\delta, \varepsilon \in F$ such that $N(\delta)=N(\varepsilon)=-N(\lambda) T(\mu)$. Now $\mu \bar{\lambda} e_{1}+\bar{\lambda} f_{1}+\delta x_{0}$ and $\mu \bar{\lambda} e_{1}+\bar{\lambda} f_{1}+\varepsilon x_{0}$ are elements of $V_{x, \lambda}$ and therefore $B$, but then $(\delta-\varepsilon) x_{0}$ is isotropic by Lemma 2.15(i), a contradiction. 
Hence $n=4$ and $\lambda=0$. Let $\lambda_{0}, \ldots, \lambda_{q}$ be a transversal for $\mathbb{F}_{q}^{*}$ in $F^{*}$. Recall that $\tau \in F^{*}$ and $\tau+\bar{\tau}=0$. For $0 \leqslant i \leqslant q$, let $\Lambda_{i}:=\left\langle\lambda_{i} e_{2}, \lambda_{i} \tau f_{2}\right\rangle_{\mathbb{F}_{q}}$ and $\Delta_{i}:=\left\{\delta e_{1}+u: \delta \in F, u \in \Lambda_{i}^{*}\right\}$. It is straightforward to verify that the orbits of $\mathrm{SU}_{4}(q)_{x}$ on $V_{x, 0} \backslash\langle x\rangle$ are $\Delta_{0}, \ldots, \Delta_{q}$ since the orbits of $\mathrm{SU}_{2}(q)$ on the isotropic vectors in $\left\langle e_{2}, f_{2}\right\rangle^{*}$ are $\Lambda_{0}^{*}, \ldots, \Lambda_{q}^{*}$. Thus $\Delta_{i} \subseteq B$ for some $i$ by Lemma 2.15(ii), in which case $\lambda_{i} e_{2}$ and $\lambda_{i} \tau f_{2}$ are elements of $B$. There exists $g \in G_{0, B}$ such that $\left(\lambda_{i} e_{2}\right)^{g}=x$. Now $z:=\left(\lambda_{i} \tau f_{2}\right)^{g} \in B \backslash\langle x\rangle$ and $\mathbf{f}(x, z)=\mathbf{f}\left(\lambda_{i} e_{2}, \lambda_{i} \tau f_{2}\right) \neq 0$, but we have already seen (replacing $y$ by $z$ above) that this leads to a contradiction.

Thus $x$ is non-isotropic. Let $\left\{v_{1}, \ldots, v_{n}\right\}$ be an orthonormal basis of $V$ (which exists by [37, Proposition 2.3.1]). We may assume without loss of generality that $x=v_{1}$. Now $B$ consists of non-isotropic vectors, so $\delta:=\mathbf{f}(y, y) \in \mathbb{F}_{q}^{*}$. Let $\lambda:=\mathbf{f}(x, y)$ and $\varepsilon:=\delta-\lambda \bar{\lambda} \in \mathbb{F}_{q}$. Define $\Gamma:=\{v \in V \backslash\langle x\rangle: \mathbf{f}(v, v)=\delta, \mathbf{f}(x, v)=\lambda\}$. Let $W:=\langle x\rangle, U:=W^{\perp}$ and $U_{\varepsilon}:=\left\{u \in U^{*}:\right.$ $\mathbf{f}(u, u)=\varepsilon\}$. Now $\Gamma=\left\{\bar{\lambda} x+u: u \in U_{\varepsilon}\right\}$. Observe that if either $n \geqslant 4$, or $n=3$ and $\varepsilon \neq 0$, then $\mathrm{SU}_{n}(q)_{x}$ acts transitively on $\Gamma$ since $\mathrm{SU}_{n-1}(q)$ acts transitively (as a subgroup of $\mathrm{SU}_{n}(q)$ ) on $U_{\varepsilon}$ by [37, Lemma 2.10.5], so $\Gamma \subseteq B$ by Lemma 2.15(ii).

First suppose that $\varepsilon=0$. By the above observations, there exist $\lambda_{2}, \lambda_{3} \in F^{*}$ such that $N\left(\lambda_{2}\right)+N\left(\lambda_{3}\right)=0$ and $z_{1}:=\bar{\lambda} x+\lambda_{2} v_{2}+\lambda_{3} v_{3} \in B$ (we may take $z_{1}=y$ when $n=3$ ). Choose $\mu \in F^{*}$ such that $\mu \neq 1$ and $\mu \bar{\mu}=1$. Let $z_{2}:=\bar{\lambda} x+\mu \lambda_{2} v_{2}+\bar{\mu} \lambda_{3} v_{3}$. There exists $g \in \operatorname{SU}_{n}(q)_{x}$ such that $v_{2}^{g}=\mu v_{2}, v_{3}^{g}=\bar{\mu} v_{3}$ and $v_{i}^{g}=v_{i}$ for $i \geqslant 4$. Now $z_{2}=z_{1}^{g} \in B$ by Lemma 2.15(ii), but $z_{2}-z_{1}$ is isotropic, contradicting Lemma 2.15(i).

Hence $\varepsilon \neq 0$ and $\Gamma \subseteq B$. It suffices to find distinct $z_{1}, z_{2} \in \Gamma$ such that $z_{2}-z_{1}$ is isotropic, for this will contradict Lemma 2.15(i). If $q=2$, then $\varepsilon=1$, so we may take $z_{1}$ and $z_{2}$ to be $\bar{\lambda} x+v_{2}$ and $\bar{\lambda} x+v_{3}$. Thus $q \geqslant 3$. Now $|K| \geqslant 3$, so there exists $\mu \in F^{*}$ such that $\mu \neq 1$ and $\mu+\bar{\mu}=2$ (including when $q$ is even). Let $F^{*}=\langle\zeta\rangle$, and write $\varepsilon=\zeta^{i(q+1)}$ and $\mu=\zeta^{j-i}$ where $1 \leqslant i \leqslant q-1$ and $1 \leqslant j-i<q^{2}-1$. Choose $\alpha \in F$ such that $\alpha \bar{\alpha}=\varepsilon-\zeta^{j} \zeta^{j q}$. Let $z_{1}:=\bar{\lambda} x+\zeta^{i} v_{2}$ and $z_{2}:=\bar{\lambda} x+\zeta^{j} v_{2}+\alpha v_{3}$. Now $z_{1}$ and $z_{2}$ are distinct elements of $\Gamma$ since $\zeta^{i} \neq \zeta^{j}$. Further,

$$
\mathbf{f}\left(z_{1}, z_{2}\right)+\mathbf{f}\left(z_{2}, z_{1}\right)-2 \lambda \bar{\lambda}=\zeta^{i} \zeta^{j q}+\zeta^{j} \zeta^{i q}=\left(\zeta^{(j-i) q}+\zeta^{j-i}\right) \zeta^{i(q+1)}=2 \varepsilon=2 \delta-2 \lambda \bar{\lambda},
$$

so $\mathbf{f}\left(z_{2}-z_{1}, z_{2}-z_{1}\right)=2 \delta-\mathbf{f}\left(z_{2}, z_{1}\right)-\mathbf{f}\left(z_{1}, z_{2}\right)=0$. Thus $z_{2}-z_{1}$ is isotropic.

\section{Class (R2)}

Let $G$ be an affine permutation group of rank 3 on $V:=V_{2 m}(q)$ for which $\Omega_{2 m}^{\varepsilon}(q) \unlhd G_{0}$ where either $m \geqslant 3$ and $\varepsilon= \pm$, or $m=2$ and $\varepsilon=-$. The orbits of $G_{0}$ on $V^{*}$ consist of the non-zero singular vectors and the non-singular vectors. In order to simplify the proofs in this section, we define a standard basis for a quadratic space. Let $Q$ be the non-degenerate quadratic form preserved by $\Omega_{2 m}^{\varepsilon}(q)$, and let $\mathbf{f}$ be the non-degenerate symmetric bilinear form associated with $Q($ so $\mathbf{f}(x, y)=Q(x+y)-Q(x)-Q(y)$ for all $x, y \in V)$. By [37, Proposition 2.5.3], if $\varepsilon=+$, then $V$ has a basis $\left\{e_{1}, \ldots, e_{m}, f_{1}, \ldots, f_{m}\right\}$ where $Q\left(e_{i}\right)=Q\left(f_{i}\right)=\mathbf{f}\left(e_{i}, e_{j}\right)=\mathbf{f}\left(f_{i}, f_{j}\right)=0$ and $\mathbf{f}\left(e_{i}, f_{j}\right)=\delta_{i, j}$ for all $i, j$. Further, if $\varepsilon=-$, then $V$ has a basis $\left\{e_{1}, \ldots, e_{m-1}, f_{1}, \ldots, f_{m-1}, x_{0}, y_{0}\right\}$ where $Q\left(e_{i}\right)=Q\left(f_{i}\right)=\mathbf{f}\left(e_{i}, e_{j}\right)=\mathbf{f}\left(f_{i}, f_{j}\right)=\mathbf{f}\left(e_{i}, x_{0}\right)=\mathbf{f}\left(f_{i}, x_{0}\right)=\mathbf{f}\left(e_{i}, y_{0}\right)=\mathbf{f}\left(f_{i}, y_{0}\right)=0$ and $\mathbf{f}\left(e_{i}, f_{j}\right)=\delta_{i, j}$ for all $i, j$, and $Q\left(x_{0}\right)=1, \mathbf{f}\left(x_{0}, y_{0}\right)=1$ and $Q\left(y_{0}\right)=\alpha$ for some $\alpha \in \mathbb{F}_{q}$ such that the polynomial $X^{2}+X+\alpha$ is irreducible over $\mathbb{F}_{q}$; we then define $e_{m}:=x_{0}$ and $f_{m}:=y_{0}$. In either case, we refer to $\left\{e_{1}, \ldots, e_{m}, f_{1}, \ldots, f_{m}\right\}$ as a standard basis of $V$.

First we consider the case where $m \geqslant 3$.

Proposition 6.1. Let $V:=V_{2 m}(q)$ where $m \geqslant 3$ and $q$ is a prime power, and let $G$ be an affine permutation group of rank 3 on $V$ where $\Omega_{2 m}^{\varepsilon}(q) \unlhd G_{0}$ and $\varepsilon= \pm$. Then there is no $\mathbb{F}_{q}$-independent $G$-affine proper partial linear space.

Proof. Let $Q$ be the non-degenerate quadratic form preserved by $\Omega_{2 m}^{\varepsilon}(q)$, let $\mathbf{f}$ be the nondegenerate symmetric bilinear form associated with $Q$, and let $\left\{e_{1}, \ldots, e_{m}, f_{1}, \ldots, f_{m}\right\}$ be a standard basis for $V$. Suppose for a contradiction that $(V, \mathcal{L})$ is an $\mathbb{F}_{q}$-independent $G$-affine 
proper partial linear space. Let $L \in \mathcal{L}_{0}$, let $B:=L^{*}$, and let $x \in L^{*}$. By Lemma 2.6, $B$ is a block of $G_{0}$ on $x^{G_{0}}$. By assumption, $B \backslash\langle x\rangle$ is non-empty.

Let $U:=\left\langle e_{1}, f_{1}\right\rangle$ and $W:=U^{\perp}$, and note that $V=U \oplus W$ since $U$ is non-degenerate. We may view $\Omega_{2 m-2}^{\varepsilon}(q)$ as a subgroup of $\Omega_{2 m}^{\varepsilon}(q)$ that fixes $\left\langle e_{1}, f_{1}\right\rangle$ pointwise and acts naturally on $W$ by [37, Lemma 4.1.1]. For $\lambda \in \mathbb{F}_{q}$, the group $\Omega_{2 m-2}^{\varepsilon}(q)$ acts transitively on $W_{\lambda}:=\left\{w \in W^{*}\right.$ : $Q(w)=\lambda\}$ by [37, Lemma 2.10.5] since $m \geqslant 3$. The following consequence of Lemma 2.15(ii) will be used repeatedly without reference below: if $x \in U$ and $u+w \in B$ where $u \in U$ and $w \in W^{*}$, then $u+z \in B$ for all $z \in W_{Q(w)}$.

First suppose that $x=e_{1}$, in which case $B$ consists of singular vectors. Choose $y \in B \backslash\langle x\rangle$, and write $y=u+w$ where $u \in U$ and $w \in W$. Since $y-x$ is singular by Lemma 2.15(i), it follows that $\mathbf{f}\left(y, e_{1}\right)=0$. Then $\mathbf{f}\left(u, e_{1}\right)=0$, so $u \in\langle x\rangle$. Since $y \notin\langle x\rangle$, it follows that $w$ is a non-zero singular vector. Thus $u+e_{2}$ and $u+f_{2}$ are elements of $B$, but then $e_{2}-f_{2}$ is singular by Lemma 2.15(i), a contradiction.

Thus we may assume that $x=e_{1}+f_{1}$, in which case $B$ consists of non-singular vectors. We claim that $B \subseteq U$. Suppose for a contradiction that there exists $y \in B \backslash U$. Write $y=u+w$ where $u \in U$ and $w \in W^{*}$. Let $\lambda:=Q(w)$. If either $\varepsilon=+$, or $\varepsilon=-$ and $m \geqslant 4$, then $u+\lambda e_{2}+f_{2}+e_{3}$ and $u+\lambda e_{2}+f_{2}$ are elements of $B$, but then $e_{3}$ is non-singular by Lemma 2.15(i), a contradiction. Thus $m=3$ and $\varepsilon=-$ (so $e_{3}$ and $f_{3}$ are non-singular). If $\lambda \neq 0$, then there exist $\mu_{1}, \mu_{2} \in \mathbb{F}_{q}$ such that $Q\left(\mu_{1} e_{3}+\mu_{2} f_{3}\right)=\lambda$ (see the remark after [37, Proposition 2.5.3]), in which case $u+e_{2}+\mu_{1} e_{3}+\mu_{2} f_{3}$ and $u+\mu_{1} e_{3}+\mu_{2} f_{3}$ are elements of $B$, but then $e_{2}$ is non-singular by Lemma 2.15(i), a contradiction. Thus $\lambda=0$. If $q>2$, then there exists $\mu \in \mathbb{F}_{q}^{*} \backslash\{1\}$, in which case $u+e_{2}$ and $u+\mu e_{2}$ are elements of $B$, but then $(\mu-1) e_{2}$ is non-singular by Lemma 2.15(i), a contradiction. Thus $q=2$. In particular, $L$ is a subspace of $V$ by Lemma 2.16. Therefore, since $u+e_{2}, u+f_{2}$ and $u+e_{2}+f_{2}+e_{3}$ are elements of $B$, it follows $u+e_{3} \in B$, but $Q\left(e_{3}\right) \neq 0$, so this is a contradiction, as above in the case $\lambda \neq 0$.

Thus $B \subseteq U$. Choose $y \in B \backslash\langle x\rangle$. In order to obtain a contradiction, it suffices to find $g \in \Omega_{2 m}^{\varepsilon}(q)_{x}$ such that $y^{g} \notin U$. There exists $g_{1} \in \mathrm{GL}_{2 m}(q)$ such that $e_{1}^{g_{1}}=e_{1}+e_{2}, f_{1}^{g_{1}}=f_{1}-e_{2}$, $e_{2}^{g_{1}}=e_{1}-f_{1}+e_{2}+f_{2}, f_{2}^{g_{1}}=e_{2}$ and $g_{1}$ fixes $\left\{e_{3}, \ldots, e_{m}, f_{3}, \ldots, f_{m}\right\}$ pointwise, and there exists $g_{2} \in \mathrm{GL}_{2 m}(q)$ that fixes $\left\{e_{1}, e_{3}, \ldots, e_{m}, f_{1}, f_{3}, \ldots, f_{m}\right\}$ pointwise and interchanges $e_{2}$ and $f_{2}$. Let $g:=g_{1}^{-1} g_{2}^{-1} g_{1} g_{2}$. Now $Q\left(v^{g_{i}}\right)=Q(v)$ for $v \in V$ and $i \in\{1,2\}$, so $g \in \Omega_{2 m}^{\varepsilon}(q)_{x}$ (see the definition of $\Omega_{2 m}^{\varepsilon}(q)$ in [37, §2.5, Descriptions 1 and 2]). Write $y=\delta_{1} e_{1}+\delta_{2} f_{1}$ where $\delta_{1}, \delta_{2} \in \mathbb{F}_{q}$, and note that $\delta_{1} \neq \delta_{2}$ since $y \notin\langle x\rangle$. Now $\left(\delta_{1} e_{1}+\delta_{2} f_{1}+\left(\delta_{2}-\delta_{1}\right) f_{2}\right)^{g_{1}}=y$, so $y^{g}=\delta_{2} e_{1}+\delta_{1} f_{1}+\left(\delta_{2}-\delta_{1}\right) e_{2} \notin U$, as desired.

In contrast to the case where $m \geqslant 3$, an $\mathbb{F}_{q}$-independent $G$-affine proper partial linear space does exist when $m=2$ and $q=3$. We describe this example now.

Example 6.2. Let $G_{0}:=M_{10} \simeq A_{6} .2 \simeq \Omega_{4}^{-}(3) .2$. Let $V$ be one of the irreducible $\mathbb{F}_{3} G_{0}$-modules of dimension 4. (There are two such modules, but the corresponding groups are conjugate in $\mathrm{GL}_{4}(3)$.) Let $G:=V: G_{0}$. Now $G$ has subdegrees 20 and 60 , and the restriction of $V$ to $H_{0}:=A_{6}$ is irreducible, so it is isomorphic to the fully deleted permutation module $D=S / W$ where $S=\left\{\left(\lambda_{1}, \ldots, \lambda_{6}\right) \in \mathbb{F}_{3}^{6}: \sum_{i=1}^{6} \lambda_{i}=0\right\}$ and $W=\left\{(\lambda, \ldots, \lambda): \lambda \in \mathbb{F}_{3}\right\}$. With this viewpoint, $H_{0}$ has orbits of sizes 20,30 and 30 containing $(1,1,1,0,0,0)+W,(1,-1,0,0,0,0)+W$ and $(1,-1,1,-1,0,0)+W$, respectively. Let $x:=(1,0,0,0,0,-1)+W$ and $B:=x^{K_{0}}$ where $K_{0}$ is the subgroup of $H_{0}$ that fixes 6 . Let $\mathcal{L}:=L^{G}$ where $L:=B \cup\{0\}$. We claim that $\mathcal{M}:=(V, \mathcal{L})$ is a $G$-affine proper partial linear space with line-size 6 and point-size 12: by Lemma 2.7, it suffices to show that $B$ is a block of $G_{0}$ and that there exists $h \in H_{L}$ for which $0^{h}=x$ where $H:=V: H_{0}$. Let $C$ be the orbit of $H_{0}$ containing $x$. Now $C$ is a block of $G_{0}$, so $G_{0, x} \leqslant G_{0, C}=H_{0}$. Thus $G_{0, x}$ is the subgroup of $H_{0}$ that fixes 1 and 6 . Since $G_{0, x} \leqslant K_{0}$, it follows that $B$ is a block of $G_{0}$. Define $h:=\tau_{v}(126)$ where $v:=(0,-1,0,0,0,1)+W$. Then $h \in H_{L}$ and $0^{h}=x$, so the claim holds. Using Magma, we verify that $\operatorname{Aut}(\mathcal{M})=G$. Note that if we instead choose $K_{0}$ to be the subgroup of $H_{0}$ that fixes 1 , then we obtain another $G$-affine proper partial linear space (to see 
this, take $h:=\tau_{v}(162)$ where $\left.v:=(-1,1,0,0,0,0)+W\right)$, but we will see in Proposition 6.3 that these partial linear spaces are isomorphic. Note also that $G_{0}$ has no proper subgroups with two orbits on $V^{*}$.

Proposition 6.3. Let $V:=V_{4}(q)$ where $q$ is a prime power, and let $G$ be an affine permutation group of rank 3 on $V$ where $\Omega_{4}^{-}(q) \unlhd G_{0}$. There is an $\mathbb{F}_{q}$-independent $G$-affine proper partial linear space $\mathcal{S}$ if and only if $q=3, G_{0}=M_{10}$ and $\mathcal{S}=\mathcal{M}$ or $\mathcal{M}^{g}$ where $g \in N_{\mathrm{GL}_{4}(3)}\left(G_{0}\right) \backslash G_{0}$ and $\mathcal{M}$ is the partial linear space of Example 6.2.

Proof. Let $S:=\Omega_{4}^{-}(q)$, and note that $S \simeq \operatorname{PSL}_{2}\left(q^{2}\right)$. Let $Q$ be the non-degenerate quadratic form preserved by $S$, let $\mathbf{f}$ be the non-degenerate symmetric bilinear form associated with $V$, and let $\left\{e_{1}, e_{2}, f_{1}, f_{2}\right\}$ be a standard basis for $V$. By [37, Lemma 2.10.5], the orbits of $S$ on $V^{*}$ are $V_{\lambda}:=\left\{v \in V^{*}: Q(v)=\lambda\right\}$ for $\lambda \in \mathbb{F}_{q}$. In particular, since $V$ contains $q\left(q^{2}+1\right)(q-1)$ non-singular vectors, it follows that $\left|V_{\lambda}\right|=q\left(q^{2}+1\right)$ for $\lambda \in \mathbb{F}_{q}^{*}$.

Let $(V, \mathcal{L})$ be an $\mathbb{F}_{q}$-independent $G$-affine proper partial linear space. Let $L \in \mathcal{L}_{0}$, let $B:=L^{*}$, and let $x \in B$. By Lemma 2.2, $G$ is primitive, so by Lemma 2.6, $B$ is a non-trivial block of $G_{0}$ on $x^{G_{0}}$. By assumption, there exists $y \in B \backslash\langle x\rangle$. If $x$ is singular, then $y$ is singular and $y \in\langle x\rangle^{\perp}$ by Lemma 2.15(i), but then $y \in\langle x\rangle$, a contradiction. Thus $x$ is non-singular, and $B$ consists of non-singular vectors.

First we claim that $S_{x} \simeq \operatorname{PSL}_{2}(q)$. Note that $\left|S_{x}\right|=q\left(q^{2}-1\right) /(2, q-1)=\left|\operatorname{PSL}_{2}(q)\right|$. If $q$ is odd, then $\langle x\rangle$ is non-degenerate, so $\Omega_{3}(q)$ is a subgroup of $S$ that fixes $\langle x\rangle$ pointwise by [37, Lemma 4.1.1(iii)]; thus $\operatorname{PSL}_{2}(q) \simeq \Omega_{3}(q) \leqslant S_{x}$, and the claim follows. Otherwise $q$ is even, in which case $S_{x}=S_{\langle x\rangle}$, and $S_{\langle x\rangle} \simeq \operatorname{Sp}_{2}(q)$ by the proof of [37, Proposition 4.1.7] (which is only stated for orthogonal groups with larger dimension). Thus $S_{x} \simeq \mathrm{PSL}_{2}(q)$, as desired.

If $q=2$, then $S$ is transitive on non-singular vectors and $S \simeq A_{5}$. By the claim, $S_{x} \simeq S_{3}$, so $S_{x}$ is maximal in $S$, in which case $B=x^{S}=x^{G_{0}}$, a contradiction. Thus $q>2$.

Let $M$ be a maximal subgroup of $S$ containing $S_{x}$. We claim that either $\left[M: S_{x}\right] \leqslant 2$, or $q=3$ and $M \simeq A_{5}$. If $q \neq 3$, then since $S_{x} \simeq \operatorname{PSL}_{2}(q)$, it follows from [7, Table 8.17] (see also Dickson [19]) that $M \simeq \mathrm{PGL}_{2}(q)$, in which case $\left[M: S_{x}\right] \leqslant 2$. If $q=3$, then $S \simeq A_{6}$ and $S_{x} \simeq A_{4}$, and the claim follows.

Let $\lambda \in \mathbb{F}_{q}^{*}$. We next claim that either $\left|B \cap V_{\lambda}\right| \leqslant 2$, or $q=3$ and $S_{B \cap V_{\lambda}} \simeq A_{5}$. Let $z \in B \cap V_{\lambda}$. Now $V_{\lambda}$ is an orbit of $S$ and $B$ is a block of $G_{0}$, so $B \cap V_{\lambda}$ is a block of $S$ in its action on $V_{\lambda}$, and $S_{z} \leqslant S_{B \cap V_{\lambda}}$. If $V_{\lambda} \subseteq B$, then some conjugate of $B$ contains $V_{1}$, but $Q\left(f_{1}+e_{2}\right)=1=Q\left(e_{2}\right)$, while $f_{1}$ is singular, contradicting Lemma 2.15(i). Thus $S_{B \cap V_{\lambda}}$ lies in some maximal subgroup $M$ of $S$. If $q=3$ and $M \simeq A_{5}$, then since $S_{z} \simeq A_{4}$, we conclude that $B \cap V_{\lambda}=\{z\}$ or $S_{B \cap V_{\lambda}} \simeq A_{5}$, as desired. Otherwise, $\left|B \cap V_{\lambda}\right| \leqslant\left[M: S_{z}\right] \leqslant 2$ by the previous claim.

We may assume that $x=e_{2}$ (since $x$ is non-singular). Suppose that $q$ is even. Let $U:=\left\langle e_{1}, f_{1}\right\rangle$ and $W:=U^{\perp}=\left\langle e_{2}, f_{2}\right\rangle$. Suppose that there exists $z \in B \backslash W$. Write $z=u+w$ where $u \in U^{*}$ and $w \in W$. Now $\left\{u^{g}+w: g \in \Omega_{2}^{+}(q)\right\} \subseteq B \cap V_{Q(z)}$ by Lemma 2.15(ii), but the orbits of $\Omega_{2}^{+}(q)$ on $U^{*}$ all have size $q-1$ by [37, Lemma 2.10.5], so $\left|B \cap V_{Q(z)}\right|>2$, a contradiction. Hence $B \subseteq W$. Now $y=\delta_{1} e_{2}+\delta_{2} f_{2}$ for some $\delta_{1}, \delta_{2} \in \mathbb{F}_{q}$ where $\delta_{2} \neq 0$. For $e \in\left\{e_{1}+e_{2}, e_{2}\right\}$, the reflection $r_{e}$ is an isometry of $(V, Q)$ for which $v \mapsto v+\mathbf{f}(e, v) e$ for all $v \in V$. By [37, p.30], $g:=r_{e_{1}+e_{2}} r_{e_{2}} \in S$ since $g$ is a product of two reflections. Now $x^{g}=x$, so $y^{g} \in B$, but $f_{2}^{g}=e_{1}+f_{2}$, so $y^{g}=\delta_{2} e_{1}+y \notin W$, a contradiction.

Thus $q$ is odd. Now $\Omega_{3}(q)=S_{x}$. Let $U:=\langle x\rangle$ and $W:=U^{\perp}$. Write $y=u+w$ where $u \in U$ and $w \in W^{*}$. Let $\lambda:=Q(y)$. Now $\left\{u+w^{g}: g \in \Omega_{3}(q)\right\} \subseteq B \cap V_{\lambda}$ by Lemma 2.15(ii), but the orbits of $\Omega_{3}(q)$ on $W^{*}$ have sizes $\left(q^{2}-1\right) / 2, q(q+1)$ and $q(q-1)$ by [37, Lemma 2.10.5], none of which are at most 2 . Thus $q=3$ and $S_{B \cap V_{\lambda}} \simeq A_{5}$. In particular, $\left|B \cap V_{\lambda}\right|=5$. Using Lemma 2.6 and Magma, we determine that this is only possible if $G_{0}=M_{10}$ and $|B|=5$. Further, $G_{0}$ has exactly two such blocks, say $B_{1}$ and $B_{2}$, and by Lemma 2.7, both $\left(B_{1} \cup\{0\}\right)^{G}$ and $\left(B_{2} \cup\{0\}\right)^{G}$ are $G$-affine proper partial linear spaces, so one must be $\mathcal{M}$, where $\mathcal{M}$ is the partial linear space of Example 6.2. Since $N_{\mathrm{GL}_{4}(3)}\left(G_{0}\right)=M_{10} .2$, the other is $\mathcal{M}^{g}$ where $g \in N_{\mathrm{GL}_{4}(3)}\left(G_{0}\right) \backslash G_{0}$. 


\section{Class (R3)}

Recall that the exterior square $\bigwedge^{2}(W)$ of a vector space $W:=V_{n}(q)$ is the quotient of $W \otimes W$ by the ideal $I:=\langle u \otimes u: u \in W\rangle$, and we write $u \wedge v$ for $u \otimes v+I$. A non-zero vector of $\bigwedge^{2}(W)$ is simple whenever it can be written in the form $u \wedge v$ for some $u, v \in W$. Note that $u \wedge u=0$ and $u \wedge v=-v \wedge u$ for all $u, v \in W$. Further, if $\left\{u_{1}, \ldots, u_{n}\right\}$ is a basis for $W$, then $\left\{u_{i} \wedge u_{j}: 1 \leqslant i<j \leqslant n\right\}$ is a basis for $\wedge^{2}(W)$. Lastly, $\Gamma \mathrm{L}(W)$ acts on $\bigwedge^{2}(W)$ by $(u \wedge v)^{g}=u^{g} \wedge v^{g}$ for all $g \in \Gamma \mathrm{L}(W)$ and $u, v \in W$. The kernel of this action is $\langle-1\rangle$ for $n \geqslant 3$.

Let $G$ be an affine permutation group of rank 3 on $V:=\bigwedge^{2}\left(V_{5}(q)\right)$ for which $\operatorname{SL}_{5}(q) \unlhd G_{0}$. The orbits of $G_{0}$ on $V^{*}$ consist of the set of simple vectors and the set of non-simple vectors.

Proposition 7.1. Let $V:=\bigwedge^{2}\left(V_{5}(q)\right)$ where $q$ is a prime power. Let $G$ be an affine permutation group of rank 3 on $V$ where $\mathrm{SL}_{5}(q) \unlhd G_{0}$. Then there is no $\mathbb{F}_{q}$-independent $G$-affine proper partial linear space.

Proof. Suppose for a contradiction that $(V, \mathcal{L})$ is an $\mathbb{F}_{q}$-independent $G$-affine proper partial linear space. Let $L \in \mathcal{L}_{0}$, let $B:=L^{*}$, and let $x \in L^{*}$. By Lemma 2.6, $B$ is a block of $G_{0}$ on $x^{G_{0}}$. By assumption, there exists $y \in B \backslash\langle x\rangle$.

First suppose that $x$ is simple. Then $x=x_{1} \wedge x_{2}$ for some linearly independent $x_{1}, x_{2} \in V_{5}(q)$ and $y=y_{1} \wedge y_{2}$ for some linearly independent $y_{1}, y_{2} \in V_{5}(q)$. If $x_{1}, x_{2}, y_{1}, y_{2}$ are linearly independent, then $y-x$ is not simple, contradicting Lemma 2.15(i), so $\mu_{1} x_{1}+\mu_{2} x_{2}+\mu_{3} y_{1}+\mu_{4} y_{2}=$ 0 for some $\mu_{1}, \ldots, \mu_{4} \in \mathbb{F}_{q}$ where $\mu_{i} \neq 0$ for some $i$. If $\mu_{3}=\mu_{4}=0$, then we have a contradiction, so without loss of generality, we may assume that $\mu_{3} \neq 0$. Hence $y=v_{1} \wedge v_{2}$ where $v_{2}:=y_{2}$ and $v_{1}:=\lambda_{1} x_{1}+\lambda_{2} x_{2}$ for some $\lambda_{1}, \lambda_{2} \in \mathbb{F}_{q}$. Now $x_{1}, x_{2}, v_{2}$ are linearly independent since $y \notin\langle x\rangle$, so we may choose $v_{3} \in V_{5}(q)$ such that $x_{1}, x_{2}, v_{2}, v_{3}$ are linearly independent, and there exists $g \in \mathrm{SL}_{5}(q)$ such that $x_{1}^{g}=x_{1}, x_{2}^{g}=x_{2}$ and $v_{2}^{g}=v_{3}$. Then $x^{g}=x$, so $z:=v_{1} \wedge v_{3}=y^{g} \in B$ by Lemma 2.15(ii). Since $v_{1}, v_{2}, v_{3}$ are linearly independent, there exists $v_{4} \in V_{5}(q)$ such that $v_{1}, v_{2}, v_{3}, v_{4}$ are linearly independent, and there exists $h \in \mathrm{SL}_{5}(q)$ such that $v_{1}^{h}=v_{3}, v_{3}^{h}=-v_{1}$ and $v_{2}^{h}=v_{4}$. Then $z^{h}=z$, so $v_{3} \wedge v_{4}=\left(v_{1} \wedge v_{2}\right)^{h} \in B$ by Lemma 2.15(ii). Thus $v_{1} \wedge v_{2}-v_{3} \wedge v_{4}$ is simple by Lemma 2.15(i), a contradiction.

Thus $x$ is not simple, in which case $x=x_{1} \wedge x_{2}+x_{3} \wedge x_{4}$ for some linearly independent $x_{1}, x_{2}, x_{3}, x_{4} \in V_{5}(q)$ and $y=y_{1} \wedge y_{2}+y_{3} \wedge y_{4}$ for some linearly independent $y_{1}, y_{2}, y_{3}, y_{4} \in$ $V_{5}(q)$. Suppose that $x_{1}, x_{2}, x_{3}, x_{4}, y_{4}$ are linearly independent. We may assume without loss of generality that $y_{1}, y_{2}, y_{3} \in\left\langle x_{1}, x_{2}, x_{3}, x_{4}\right\rangle$. Choose $x_{0} \in\left\langle x_{1}, x_{2}, x_{3}, x_{4}\right\rangle \backslash\left\langle y_{3}\right\rangle$. There exists $g \in \mathrm{SL}_{5}(q)$ such that $x_{i}^{g}=x_{i}$ for $1 \leqslant i \leqslant 4$ and $y_{4}^{g}=x_{0}+y_{4}$. Then $x^{g}=x$ and $y^{g}-y=y_{3} \wedge x_{0} \neq 0$, contradicting Lemma 2.15. By symmetry, we conclude that $y_{i} \in\left\langle x_{1}, x_{2}, x_{3}, x_{4}\right\rangle$ for $1 \leqslant i \leqslant 4$. Now $y=\sum_{1 \leqslant i<j \leqslant 4} \lambda_{i, j} x_{i} \wedge x_{j}$ for some $\lambda_{i, j} \in \mathbb{F}_{q}$. Let $x_{0}:=\lambda_{1,3} x_{3}+\lambda_{1,4} x_{4}$. There exists $h \in \operatorname{SL}_{5}(q)$ such that $x_{1}^{h}=x_{1}+x_{2}$ and $x_{i}^{h}=x_{i}$ for $2 \leqslant i \leqslant 4$. Now $x^{h}=x$ and $y^{h}-y=x_{2} \wedge x_{0}$, so by Lemma $2.15, x_{0}=0$. By exchanging the roles of $x_{1}$ and $x_{2}$ in this argument, we see that $y=\lambda x_{1} \wedge x_{2}+\mu x_{3} \wedge x_{4}$ for some $\lambda, \mu \in \mathbb{F}_{q}$. Note that $\lambda \neq \mu$ since $y \notin\langle x\rangle$. There exists $k \in \mathrm{SL}_{5}(q)$ such that $x_{1}^{k}=x_{1}+x_{3}, x_{4}^{k}=x_{4}-x_{2}$ and $x_{i}^{k}=x_{i}$ for $i=2,3$. Then $x^{k}=x$ and $y^{k}-y=(\lambda-\mu) x_{3} \wedge x_{2} \neq 0$, contradicting Lemma 2.15 .

\section{Class (R4)}

Let $G$ be an affine permutation group of rank 3 on $V_{4}(q)$ for which $\operatorname{Sz}(q) \unlhd G_{0}$, where $q=2^{2 n+1}$. Recall that the group $G$ has suborbits of size $(q-1)\left(q^{2}+1\right)$ and $q(q-1)\left(q^{2}+1\right)$.

Proposition 8.1. Let $V:=V_{4}(q)$ where $q:=2^{2 n+1}$ for some $n \geqslant 1$, and let $G$ be an affine permutation group of rank 3 on $V$ where $\mathrm{Sz}(q) \unlhd G_{0}$. Then there is no $\mathbb{F}_{q}$-independent $G$-affine proper partial linear space. 
Proof. We follow the notation of [53]. Let $\theta \in \operatorname{Aut}\left(\mathbb{F}_{q}\right)$ where $\alpha^{\theta}=\alpha^{2^{n+1}}$ for all $\alpha \in \mathbb{F}_{q}$. For $\alpha, \beta \in \mathbb{F}_{q}$, define $f(\alpha, \beta):=\alpha^{\theta+2}+\alpha \beta+\beta^{\theta}$,

$$
[\alpha, \beta]:=\left(\begin{array}{cccc}
1 & 0 & 0 & 0 \\
\alpha & 1 & 0 & 0 \\
\alpha^{\theta+1}+\beta & \alpha^{\theta} & 1 & 0 \\
f(\alpha, \beta) & \beta & \alpha & 1
\end{array}\right), \tau:=\left(\begin{array}{cccc}
0 & 0 & 0 & 1 \\
0 & 0 & 1 & 0 \\
0 & 1 & 0 & 0 \\
1 & 0 & 0 & 0
\end{array}\right),
$$

and observe that $[\alpha, \beta][\gamma, \delta]=\left[\alpha+\gamma, \alpha \gamma^{\theta}+\beta+\delta\right]$ for all $\gamma, \delta \in \mathbb{F}_{q}$. We also define

$$
\begin{aligned}
Q(q) & :=\left\{[\alpha, \beta]: \alpha, \beta \in \mathbb{F}_{q}\right\}, \\
K(q) & :=\left\{\operatorname{diag}\left(\kappa^{\theta^{-1}+1}, \kappa^{\theta^{-1}},\left(\kappa^{\theta^{-1}}\right)^{-1},\left(\kappa^{\theta^{-1}+1}\right)^{-1}\right): \kappa \in \mathbb{F}_{q}^{*}\right\}, \\
H(q) & :=\langle Q(q), K(q)\rangle, \\
\operatorname{Sz}(q) & :=\langle H(q), \tau\rangle .
\end{aligned}
$$

Clearly $Q(q)$ is a group of order $q^{2}$. Note that if $\kappa^{\theta+1}=1$ for some $\kappa \in \mathbb{F}_{q}^{*}$, then $\kappa=1$ since the integers $q-1$ and $2^{n+1}+1$ are coprime, so $\left\{\kappa^{\theta+1}: \kappa \in \mathbb{F}_{q}^{*}\right\}=\mathbb{F}_{q}^{*}$. Now $K(q)$ is a cyclic group of order $q-1$, and $K(q)$ normalises $Q(q)$, so $H(q)=Q(q): K(q)$ and $|H(q)|=q^{2}(q-1)$. Let $\operatorname{Sz}(q)$ act on $V$ with respect to the basis $\left\{v_{1}, v_{2}, v_{3}, v_{4}\right\}$. By [53, Theorem 7] and its proof, $\operatorname{Sz}(q)$ has order $q^{2}(q-1)\left(q^{2}+1\right)$ and every element of $\mathrm{Sz}(q) \backslash H(q)$ has the form $h_{1} \tau h_{2}$ for some $h_{1} \in H(q)$ and $h_{2} \in Q(q)$. It follows that $\operatorname{Sz}(q)_{v_{1}}=Q(q)$ and

$$
\Omega_{1}:=v_{1}^{\mathrm{Sz}(q)}=\left\{\lambda v_{1}: \lambda \in \mathbb{F}_{q}^{*}\right\} \cup\left\{\mu\left(f(\alpha, \beta) v_{1}+\beta v_{2}+\alpha v_{3}+v_{4}\right): \mu \in \mathbb{F}_{q}^{*}, \alpha, \beta \in \mathbb{F}_{q}\right\},
$$

a set of size $(q-1)\left(q^{2}+1\right)$. Moreover, $\operatorname{Sz}(q)_{v_{2}}=\left\{[0, \beta]: \beta \in \mathbb{F}_{q}\right\}$ and $\Omega_{2}:=v_{2}^{\mathrm{Sz}(q)}$ is a set of size $q(q-1)\left(q^{2}+1\right)$. Hence $V: \mathrm{Sz}(q)$ is itself a rank 3 permutation group on $V$.

Suppose for a contradiction that $(V, \mathcal{L})$ is an $\mathbb{F}_{q}$-independent $G$-affine proper partial linear space. Then $(V, \mathcal{L})$ is also a $(V: \mathrm{Sz}(q))$-affine partial linear space. Let $L \in \mathcal{L}_{0}$, let $B:=L^{*}$, and let $x \in B$. By Lemma 2.2, $V: \mathrm{Sz}(q)$ is primitive, so by Lemma 2.6, $B$ is a non-trivial block of $\mathrm{Sz}(q)$ on $x^{\mathrm{Sz}(q)}$. By assumption, there exists $y \in B \backslash\langle x\rangle$. Now $y=\sum_{i=1}^{4} \lambda_{i} v_{i}$ for some $\lambda_{i} \in \mathbb{F}_{q}$. If $x=v_{1}$, then $y \in \Omega_{1} \backslash\left\langle v_{1}\right\rangle$, so $\lambda_{4} \neq 0$, but $x^{[0,1]}=x$ and $y^{[0,1]}-y=\left(\lambda_{3}+\lambda_{4}\right) v_{1}+\lambda_{4} v_{2} \notin x^{\mathrm{Sz}(q)}$, contradicting Lemma 2.15. It follows that $x \notin \Omega_{1}$, so $x \in \Omega_{2}$, and we may assume without loss of generality that $x=v_{2}$.

Observe that $\mathrm{Sz}(q)_{x} \leqslant \mathrm{Sz}(q)_{B}<\mathrm{Sz}(q)$. Now $q$ divides $\left|\mathrm{Sz}(q)_{B}\right|$, so $\mathrm{Sz}(q)_{B} \leqslant H(q)^{g}$ for some $g \in \mathrm{Sz}(q)$ by [53, Theorem 9]. If $g \notin H(q)$, then $H(q)^{g}=H(q)^{\tau h}$ for some $h \in Q(q)$, but $\mathrm{Sz}(q)_{x}=Z(Q(q))$, so $\mathrm{Sz}(q)_{x} \leqslant H(q)^{\tau}$, a contradiction since $\tau$ conjugates any lower triangular matrix to an upper triangular matrix. Hence $g \in H(q)$, so $\operatorname{Sz}(q)_{B} \leqslant H(q)$.

Let $C:=\left\langle v_{1}, v_{2}\right\rangle \backslash\left\langle v_{1}\right\rangle$. Now $\operatorname{Sz}(q)_{C}=H(q)$ and $C$ is a block of $\operatorname{Sz}(q)$ in its action on $\Omega_{2}$. Thus $B \subseteq C$. In particular, $|B|$ divides $q(q-1)$. Moreover, $L$ is an $\mathbb{F}_{2}$-subspace of $V$ by Lemma 2.16, so $|B|$ divides $q-1$. Observe that $\mathrm{Sz}(q)_{x}$ is the kernel of the action of $H(q)$ on $C$, so $\bar{H}:=H(q) / \mathrm{Sz}(q)_{x}$ acts regularly on $C$. Thus $\left|\bar{H}_{B}\right|$ divides $q-1$.

Let $\bar{Q}:=Q(q) / \mathrm{Sz}(q)_{x}$ and $\bar{K}:=K(q) \mathrm{Sz}(q)_{x} / \mathrm{Sz}(q)_{x} \simeq K(q)$. Note that $\bar{H}=\bar{Q}: \bar{K}$. Further, $|\bar{Q}|=q$ and $|\bar{K}|=q-1$. Now $\bar{H}_{B} \cap \bar{Q}=1$. In particular, $\bar{H}_{B}$ is isomorphic to a subgroup of $\bar{H} / \bar{Q} \simeq \bar{K}$, so $\bar{H}_{B}$ is cyclic. Let $h$ be a generator of $\bar{H}_{B}$. We claim that $h \in \bar{K}^{g}$ for some $g \in \bar{Q}$. Observe that $\bar{K} \cap \bar{K}^{g}=1$ for all $g \in \bar{Q} \backslash\{1\}$, so

$$
\left|\bar{H} \backslash\left(\bigcup_{g \in \bar{Q}} \bar{K}^{g}\right)\right|=|\bar{H}|-(|\bar{Q}|(|\bar{K}|-1)+1)=|\bar{Q}|-1 .
$$

Thus $\bar{Q} \backslash\{1\}=\bar{H} \backslash\left(\bigcup_{g \in \bar{Q}} \bar{K}^{g}\right)$, and the claim follows.

Hence $\bar{H}_{B} \leqslant \bar{K}^{g}$ for some $g \in \bar{Q}$. It follows that $B \subseteq\left\{\alpha\left(\kappa^{\theta^{-1}+1}+\kappa^{\theta^{-1}}\right) v_{1}+\kappa^{\theta^{-1}} v_{2}: \kappa \in \mathbb{F}_{q}^{*}\right\}$ for some $\alpha \in \mathbb{F}_{q}$. Note that $\alpha \neq 0$, or else $B \subseteq\langle x\rangle$. Since $|B| \geqslant 2$, there exists $\kappa \in \mathbb{F}_{q}^{*}$ 
such that $\kappa \neq 1$ and $\alpha\left(\kappa^{\theta^{-1}+1}+\kappa^{\theta^{-1}}\right) v_{1}+\kappa^{\theta^{-1}} v_{2} \in B$, and since $L$ is an $\mathbb{F}_{2}$-subspace of $V$, $\alpha\left(\kappa^{\theta^{-1}+1}+\kappa^{\theta^{-1}}\right) v_{1}+\left(\kappa^{\theta^{-1}}+1\right) v_{2} \in B$. Note that $\kappa^{\theta^{-1}}+1=(\kappa+1)^{\theta^{-1}}$. Now

$$
\kappa^{\theta^{-1}+1}+\kappa^{\theta^{-1}}=(\kappa+1)^{\theta^{-1}+1}+(\kappa+1)^{\theta^{-1}}=\kappa^{\theta^{-1}+1}+\kappa .
$$

Thus $\kappa^{\theta}=\kappa$, but then $\kappa=\kappa^{\theta}=\kappa^{\theta^{2}}=\kappa^{2}$, so $\kappa=1$, a contradiction.

\section{Class (R5)}

In order to determine the $G$-affine proper partial linear spaces where $G$ belongs to (R5), we first outline the theory of spin modules and the quasisimple group $\operatorname{Spin}_{m}^{\varepsilon}(q)$. Our exposition closely follows Chevalley's treatise on the subject [13].

Let $W:=V_{m}(q)$ where $m \geqslant 7$ and $q$ is a prime power. Let $Q$ be a non-degenerate quadratic form on $W$ with associated non-degenerate symmetric bilinear form $\mathbf{f}$. (In particular, if $m$ is odd, then $q$ is odd.) Let $\varepsilon$ be the type of $Q$, where $\varepsilon \in\{+,-, \circ\}$. The Clifford algebra $C(W, Q)=C$ is defined to be the algebra $T / I$, where $T$ is the tensor algebra of $W$ (see [51]) and $I$ is the ideal generated by $v \otimes v-Q(v)$ for all $v \in W$. We view $W$ as a subspace of $C$ by identifying each $v \in W$ with $v+I$. Similarly, we view $\mathbb{F}_{q}$ as a subalgebra of $C$ by identifying each $\lambda \in \mathbb{F}_{q}$ with $\lambda+I$. We also suppress the notation $\otimes$. Now $v^{2}=Q(v)$ and $v w+w v=\mathbf{f}(v, w)$ for all $v, w \in V$.

By [13, II.1.2], for any basis $\left\{v_{1}, \ldots, v_{m}\right\}$ of $W$, the Clifford algebra has a basis consisting of the products $v_{i_{1}} \cdots v_{i_{k}}$ for $1 \leqslant k \leqslant m$ and integers $i_{1}, \ldots, i_{k}$ such that $1 \leqslant i_{1}<\cdots<i_{k} \leqslant m$, as well as the empty product 1 . In particular, $C$ has dimension $2^{m}$. Further, $C=C_{+} \oplus C_{-}$where $C_{+}$ (respectively, $C_{-}$) is the subspace of $C$ generated by the basis vectors with an even (respectively, odd) number of factors. We call the elements of $C_{+}$and $C_{-}$even and odd, respectively. Let $Z(C)$ denote the centre of $C$. For $m$ even, $Z(C)=\mathbb{F}_{q}$ by [13, II.2.1], and for $m$ odd, $Z(C)=\mathbb{F}_{q}+\mathbb{F}_{q} z_{0}$ for some $z_{0} \in C_{-}$by [13, II.2.6]. In particular, $Z(C) \cap C_{+}=\mathbb{F}_{q}$. For $R \subseteq C$, we write $R^{\times}$for the set of invertible elements in $R$.

The Clifford group $\Gamma(C)=\Gamma$ is defined to be $\left\{s \in C^{\times}: s^{-1} x s \in W\right.$ for all $\left.x \in W\right\}$. Note that $\Gamma \cap W$ is the set of non-singular vectors of $W$, for such a vector $v$ has inverse $Q(v)^{-1} v$ and $v^{-1} x v=-x+\mathbf{f}(x, v) Q(v)^{-1} v$ for all $x \in W$, while no singular vector of $W$ is invertible. The even Clifford group $\Gamma^{+}$is defined to be $\Gamma \cap C_{+}$. There is a natural representation $\chi$ of $\Gamma$ on $W$ defined by $s \chi: x \mapsto s^{-1} x s$ for $s \in \Gamma$ and $x \in W$. By [13, II.3.1], $\chi$ has kernel $Z(C)^{\times}$, and $\Gamma \chi$ is $\mathrm{O}_{m}^{\varepsilon}(q)$ for $m$ even and $\mathrm{SO}_{m}^{\varepsilon}(q)$ for $m$ odd. By [13, II.3.3], the group $\Gamma^{+} \chi$ has index 2 in $\mathrm{O}_{m}^{\varepsilon}(q)$ and, for $q$ odd, equals $\mathrm{SO}_{m}^{\varepsilon}(q)$. Note that $v \chi=-r_{v}$ for all non-singular vectors $v \in W$, where $r_{v}$ is the reflection in $v$, defined by $x \mapsto x-\mathbf{f}(x, v) Q(v)^{-1} v$ for all $x \in W$.

The Clifford algebra has an antiautomorphism $\alpha$ defined by $v_{1} v_{2} \cdots v_{k} \mapsto v_{k} v_{k-1} \cdots v_{1}$ for all $k \geqslant 1$ and $v_{i} \in W$ (see [51]). Now $\Gamma^{\alpha}=\Gamma$ and $s\left(s^{\alpha}\right) \in Z(C)^{\times}$for $s \in \Gamma$ by [13, II.3.5], so there is a homomorphism $\varphi: \Gamma \rightarrow Z(C)^{\times}$defined by $s \mapsto s\left(s^{\alpha}\right)$ for $s \in \Gamma$. Note that $v \varphi=v^{2}=Q(v)$ for $v \in \Gamma \cap W$. Let $\Gamma_{0}:=\operatorname{ker}(\varphi)$ and $\Gamma_{0}^{+}:=\Gamma_{0} \cap C_{+}$. Let $\pi: \mathbb{F}_{q}^{*} \rightarrow \mathbb{F}_{q}^{*} / \mathbb{F}_{q}^{* 2}$ be the natural homomorphism, where $\mathbb{F}_{q}^{* 2}=\left\{\lambda^{2}: \lambda \in \mathbb{F}_{q}^{*}\right\}$, and note that $\left|\mathbb{F}_{q}^{*} / \mathbb{F}_{q}^{* 2}\right|=\operatorname{gcd}(2, q-1)$. Since $\operatorname{ker}\left(\left.\chi\right|_{\Gamma^{+}}\right)=\mathbb{F}_{q}^{*} \leqslant \operatorname{ker}(\varphi \pi)$, there is a homomorphism $\theta: \Gamma^{+} \chi \rightarrow \mathbb{F}_{q}^{*} / \mathbb{F}_{q}^{* 2}$ called the spinor norm defined by $s \chi \mapsto s \varphi \pi$ for $s \in \Gamma^{+}$. Now $\left(\operatorname{ker}\left(\left.\varphi\right|_{\Gamma^{+}}\right)\right) \chi \leqslant \operatorname{ker}(\theta)$, and $\operatorname{ker}(\theta) \leqslant\left(\operatorname{ker}\left(\left.\varphi\right|_{\Gamma^{+}}\right)\right) \chi$ since $\operatorname{ker}(\pi) \leqslant\left(\operatorname{ker}\left(\left.\chi\right|_{\Gamma^{+}}\right)\right) \varphi$. Thus $\Gamma_{0}^{+} \chi=\operatorname{ker}(\theta)$. It follows from the definition of $\Omega_{m}^{\varepsilon}(q)$ in [37, $\S 2.5$, Descriptions 1 and 2] that $\operatorname{ker}(\theta)=\Omega_{m}^{\varepsilon}(q)$. Thus $\Gamma_{0}^{+} \chi=\Omega_{m}^{\varepsilon}(q)$. Further, $\Gamma_{0}^{+} \cap \operatorname{ker}(\chi)=\langle-1\rangle$ since $\operatorname{ker}\left(\left.\chi\right|_{\Gamma^{+}}\right)=\mathbb{F}_{q}^{*}$. Hence $\Gamma_{0}^{+} /\langle-1\rangle \simeq \Omega_{m}^{\varepsilon}(q)$. The group $\Gamma_{0}^{+}$is called the spin group and is also denoted by $\operatorname{Spin}_{m}^{\varepsilon}(q)$. Since $\Omega_{m}^{\varepsilon}(q)$ is perfect, $\Gamma_{0}^{+}=\left(\Gamma_{0}^{+}\right)^{\prime}\langle-1\rangle$. There exist $u, v \in W$ such that $Q(u)=Q(v)=1$ and $\mathbf{f}(u, v)=0$. Now $u v \in \Gamma_{0}^{+}$, so $-1=(u v)^{2} \in\left(\Gamma_{0}^{+}\right)^{\prime}$. Thus $\operatorname{Spin}_{m}^{\varepsilon}(q)$ is perfect. The proof of the following is routine.

Lemma 9.1. Let $Q$ be a non-degenerate quadratic form on $W:=V_{m}(q)$ with type $\varepsilon$ and associated bilinear form $\mathbf{f}$ where $m \geqslant 7$ and $q$ is a prime power. Let $u, v \in W$ be linearly independent where $Q(u)=0$ and $\mathbf{f}(u, v)=0$. Then $(1-u v)(1+u v)=1$ and $(1+u v) x(1-u v)=$ $x+\mathbf{f}(x, v) u-\mathbf{f}(x, u) v-Q(v) \mathbf{f}(x, u) u$ for all $x \in W$. In particular, $1-u v \in \operatorname{Spin}_{m}^{\varepsilon}(q)$. 
Now suppose that $m$ is even and $\varepsilon=+$. Write $m=2 r$. Let $\left\{e_{1}, \ldots, e_{r}, f_{1}, \ldots, f_{r}\right\}$ be a standard basis of $W$ (see $\S 6$ ). Let $E:=\left\langle e_{1}, \ldots, e_{r}\right\rangle$, and denote by $C^{E}$ the subalgebra of $C$ generated by $E$. For $S=\left\{i_{1}, \ldots, i_{k}\right\}$ in the power set $\mathbb{P}(\{1, \ldots, r\})$ where $i_{1}<\cdots<i_{k}$, let $e_{S}:=e_{i_{1}} \cdots e_{i_{k}}$ and $f_{S}:=f_{i_{1}} \cdots f_{i_{k}}$. Let $e_{\varnothing}:=1$ and $f_{\varnothing}:=1$. Then $\left\{e_{S}: S \in \mathbb{P}(\{1, \ldots, r\})\right\}$ is a basis for $C^{E}$, and $\left\{f_{T} e_{S}: T, S \in \mathbb{P}(\{1, \ldots, r\})\right\}$ is a basis for $C$. Let $f^{*}:=f_{1} \cdots f_{r}=f_{\{1, \ldots, r\}}$, and note that $f^{*} f_{i}=0$ for $1 \leqslant i \leqslant r$. Using this observation and the above basis for $C$, it can be verified that, for each $a \in C^{E}$ and $c \in C$, there exists a unique $a^{\prime} \in C^{E}$ such that $f^{*} a c=f^{*} a^{\prime}$, and we write $a^{c}:=a^{\prime}$. Further, $C^{E}$ is a right $C$-module under this action. Define $C_{+}^{E}:=C^{E} \cap C^{+}$. Now $C_{+}^{E}$ is an irreducible $\mathbb{F}_{q} \Gamma_{0}^{+}$-module by [13, II.4.3] and the introduction to $\left[13, \mathrm{Ch}\right.$. III]; it is called the spin module and has dimension $2^{r-1}$. The kernel of the action of $\Gamma^{+}$on $C_{+}^{E}$ has order $\operatorname{gcd}(2, q-1)$ by [13, III.6.1]. Let $z:=\Pi_{i=1}^{r}\left(e_{i}+f_{i}\right)\left(f_{i}-e_{i}\right)$. For $S \subseteq\{1, \ldots, r\}$, note that $f^{*} e_{S}\left(e_{i}+f_{i}\right)\left(f_{i}-e_{i}\right)$ is $-f^{*} e_{S}$ when $i \in S$ and $f^{*} e_{S}$ otherwise, whence $e_{S}^{z}=(-1)^{|S|} e_{S}$. Since those $e_{S}$ with $|S|$ even form a basis of $C_{+}^{E}$, it follows that $\langle z\rangle$ is the kernel of $\Gamma^{+}$on $C_{+}^{E}$. In particular, for $r$ odd, $C_{+}^{E}$ is a faithful $\mathbb{F}_{q} \operatorname{Spin}_{2 r}^{+}(q)$-module.

We claim that $\operatorname{Spin}_{10}^{+}(q)$ is quasisimple with centre $\operatorname{Spin}_{10}^{+}(q) \cap Z$ where $Z:=Z\left(\operatorname{GL}\left(C_{+}^{E}\right)\right)$. Since $\Gamma_{0}^{+} /\langle-1\rangle \simeq \Omega_{10}^{+}(q)$, it suffices to consider the case where $\Omega_{10}^{+}(q)$ is not simple, so we assume that $q \equiv 1 \bmod 4$. Now there exists $\lambda \in \mathbb{F}_{q}^{*}$ such that $\lambda^{2}=-1$, and $z \lambda \in \Gamma_{0}^{+} \cap Z$. Since $\left(\Gamma_{0}^{+} \cap Z\right) /\langle-1\rangle \leqslant Z\left(\Gamma_{0}^{+}\right) /\langle-1\rangle \leqslant Z\left(\Omega_{10}^{+}(q)\right)$, which has order 2 , it follows that $\Gamma_{0}^{+} \cap Z=Z\left(\Gamma_{0}^{+}\right)$ and that $\Gamma_{0}^{+}$is quasisimple.

Let $M$ be a maximal totally singular subspace of $W$. By $[13, \S 3.1]$, there exists a unique one-dimensional subspace $P_{M}$ of $C^{E}$ such that $v^{w}=0$ for all $v \in P_{M}$ and $w \in M$. Any non-zero element of $P_{M}$ is called a representative spinor of $M$; for example, 1 is a representative spinor of $\left\langle f_{1}, \ldots, f_{r}\right\rangle$. Any element of $C^{E}$ that is the representative spinor of some maximal totally singular subspace is called a pure spinor. If $u$ is a representative spinor of the maximal totally singular subspace $M^{\prime}$, then $M^{\prime}=\left\{v \in W: u^{v}=0\right\}$ by [13, III.1.4]. Thus there is a one-to-one correspondence between the maximal totally singular subspaces of $W$ and the one-dimensional subspaces of $C^{E}$ spanned by pure spinors. If $u$ is a representative spinor of $M$ and $s \in \Gamma$, then $u^{s}$ is a representative spinor of $s^{-1} M s$ by [13, III.1.3]. The group $\Gamma_{0} \chi$ acts transitively on the maximal totally singular subspaces by [13, III.2.7], so $\Gamma_{0}$ acts transitively on the one-dimensional subspaces of $C^{E}$ spanned by pure spinors. Since $m$ is even, any element $s$ of $\Gamma$ is either even or odd by [13, II.3.2], so $1^{s}$ is either even or odd. Thus a pure spinor is either even or odd, and $\Gamma_{0}^{+}$acts transitively on the one-dimensional subspaces of $C_{+}^{E}$ spanned by pure spinors. Further, since $W$ has exactly $2(q+1)\left(q^{2}+1\right) \cdots\left(q^{r-1}+1\right)$ maximal totally singular subspaces, there are exactly $(q-1)(q+1)\left(q^{2}+1\right) \cdots\left(q^{r-1}+1\right)$ pure spinors in $C_{+}^{E}$. The following is [13, III.1.12].

Lemma 9.2. Let $M$ and $M^{\prime}$ be distinct maximal totally singular subspaces of $V_{2 r}(q)$ with respect to a non-degenerate quadratic form with type + where $r \geqslant 4$ and $q$ is a prime power. Let $u$ and $u^{\prime}$ be representative spinors of $M$ and $M^{\prime}$, respectively. Then $u+u^{\prime}$ is pure if and only if $\operatorname{dim}\left(M \cap M^{\prime}\right)=r-2$.

Observe that $\left\{e_{S}: S \in \mathbb{P}(\{1, \ldots, r\}),|S| \equiv 0 \bmod 2\right\}$ is a basis for $C_{+}^{E}$ consisting entirely of pure spinors. Indeed, $e_{S}$ is a representative spinor for the maximal totally singular subspace spanned by $e_{i}$ for $i \in S$ and $f_{j}$ for $j \in\{1, \ldots, r\} \backslash S$.

Lemma 9.3. Let $Q$ be a non-degenerate quadratic form on $W:=V_{2 r}(q)$ with type + where $r \geqslant 4$ and $q$ is a power of a prime $p$. Let $\left\{e_{1}, \ldots, e_{r}, f_{1}, \ldots, f_{r}\right\}$ be a standard basis of $W$, and let $E:=\left\langle e_{1}, \ldots, e_{r}\right\rangle$. Let $H \leqslant \operatorname{Spin}_{2 r}^{+}(q)$, and let $B$ be a block of $H$ on $C_{+}^{E}$ such that $B \cup\{0\}$ is an $\mathbb{F}_{p}$-subspace of $C_{+}^{E}$. Let $x, y \in B$. Let $I:=\{S \in \mathbb{P}(\{1, \ldots, r\}):|S| \equiv 0 \bmod 2\}$ and write $y=\sum_{S \in I} \lambda_{S} e_{S}$ where $\lambda_{S} \in \mathbb{F}_{q}$ for $S \in I$. Let $\ell, k \in\{1, \ldots, r\}$ where $\ell \neq k$, and let $I_{\ell, k}:=\{S \in I: \ell \in S, k \notin S\}$. If $\lambda_{T} \neq 0$ for some $T \in I_{\ell, k}$ and $1-f_{\ell} e_{k} \in H_{x}$, then

$$
\sum_{S \in I_{\ell, k}}(-1)^{i_{S}} \lambda_{S} e_{(S \backslash\{\ell\}) \cup\{k\}} \in B
$$


for some $i_{S} \in\{0,1\}$.

Proof. Let $f^{*}:=f_{1} \cdots f_{r}$ and $s:=1-f_{\ell} e_{k}$. Now $f^{*} y^{s}=f^{*} y-\sum_{S \in I_{\ell, k}} \lambda_{S} f^{*} e_{S} f_{\ell} e_{k}$ since $f^{*} e_{S} f_{\ell} e_{k}=0$ for $S \in I \backslash I_{\ell, k}$. Further, for $S \in I_{\ell, k}, f^{*} e_{S} f_{\ell} e_{k}=f^{*}(-1)^{i} e_{(S \backslash\{\ell\}) \cup\{k\}}$ for some $i_{S} \in\{0,1\}$ since for any $A \in \mathbb{P}(\{1, \ldots, r\})$ with $\ell \notin A, f^{*} e_{A} e_{\ell} f_{\ell}=f^{*} e_{A}\left(1-f_{\ell} e_{\ell}\right)=f^{*} e_{A}$. Let $w:=\sum_{S \in I_{\ell, k}}(-1)^{i_{S}} \lambda_{S} e_{(S \backslash\{\ell\}) \cup\{k\}} \neq 0$. Now $x^{s}=x$, so $y-w=y^{s} \in B$. Thus $w \in B$.

Now we consider class $(\mathrm{R} 5)$ where $(m, n, \varepsilon)=(10,16,+)$.

Proposition 9.4. Let $V$ be the spin module of $\operatorname{Spin}_{10}^{+}(q)$ where $q$ is a prime power. Let $G$ be an affine permutation group of rank 3 on $V$ where $\operatorname{Spin}_{10}^{+}(q) \unlhd G_{0}$. Then there is no $\mathbb{F}_{q}$-independent $G$-affine proper partial linear space.

Proof. Let $Q$ be a non-degenerate quadratic form on $W:=V_{10}(q)$ with type + and standard basis $\left\{e_{1}, \ldots, e_{5}, f_{1}, \ldots, f_{5}\right\}$. Let $E:=\left\langle e_{1}, \ldots, e_{5}\right\rangle$ so that $V=C_{+}^{E}$, and let $Z:=Z\left(\operatorname{GL}\left(C_{+}^{E}\right)\right)$. Recall that $\operatorname{Spin}_{10}^{+}(q) /\left(\operatorname{Spin}_{10}^{+}(q) \cap Z\right) \simeq \mathrm{P} \Omega_{10}^{+}(q)$ and that $\operatorname{Spin}_{10}^{+}(q) Z$ acts transitively on the set of pure spinors in $C_{+}^{E}$. By [39, Lemma 2.9], $\operatorname{Spin}_{10}^{+}(q) Z$ has two orbits on $\left(C_{+}^{E}\right)^{*}$. Thus one of the orbits of $G_{0}$ is the set of pure spinors. Let $q=p^{e}$ where $p$ is prime.

Suppose for a contradiction that $\left(C_{+}^{E}, \mathcal{L}\right)$ is an $\mathbb{F}_{q}$-independent $G$-affine proper partial linear space. Let $L \in \mathcal{L}_{0}$, let $B:=L^{*}$, and let $x \in B$. By Lemma 2.6, $B$ is a block of $G_{0}$ on $x^{G_{0}}$, and $L$

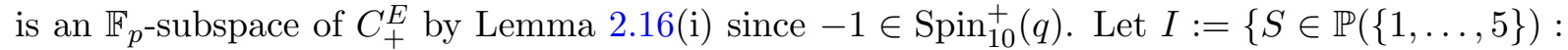
$|S| \equiv 0 \bmod 2\}$, and for distinct integers $\ell, k \in\{1,2,3,4,5\}$, let $I_{\ell, k}:=\{S \in I: \ell \in S, k \notin S\}$. By assumption, there exists $y \in B \backslash\langle x\rangle$. Write $y=\sum_{S \in I} \lambda_{S} e_{S}$ where $\lambda_{S} \in \mathbb{F}_{q}$ for $S \in I$.

First suppose that $x=1$. Since $y \notin\langle 1\rangle$, there exists $T \in I \backslash\{\varnothing\}$ such that $\lambda_{T} \neq 0$. Since $T$ is a non-empty proper subset of $\{1, \ldots, 5\}$, we may choose $k \in\{1, \ldots, 5\} \backslash T$ and $\ell \in T$. Now $T \in I_{\ell, k}$ and $1-f_{\ell} e_{k} \in \operatorname{Spin}_{10}^{+}(q)_{x}$ by Lemma 9.1, so Lemma 9.3 implies that $w:=\sum_{S \in I_{\ell, k}}(-1)^{i_{S}} \lambda_{S} e_{(S \backslash\{\ell\}) \cup\{k\}} \in B$ for some $i_{S} \in\{0,1\}$. Thus $w=\sum_{j \neq \ell, k} \mu_{j} e_{\{j, k\}}+\mu e_{A}$ for some $\mu_{j}, \mu \in \mathbb{F}_{q}$ where $A:=\{1, \ldots, 5\} \backslash\{\ell\}$. First suppose that $w=\mu e_{A}$. Then $1+\mu e_{A} \in B$. However, $1+\mu e_{A}$ is not pure by Lemma 9.2, a contradiction. Thus $\mu_{j} \neq 0$ for some $j$ (where $j \neq \ell, k)$. Choose $i \in\{1, \ldots, 5\} \backslash\{\ell, k, j\}$, and note that $i \in A$. Let $\ell^{*}:=j$ and $k^{*}:=i$. Now $1-f_{\ell^{*}} e_{k^{*}} \in \operatorname{Spin}_{10}^{+}(q)_{x}$ by Lemma 9.1, and $\{j, k\}$ is the only element of $I_{\ell^{*}, k^{*}}$ in the decomposition of $w$, so by Lemma $9.3,\left\langle e_{\{i, k\}}\right\rangle \cap B \neq \varnothing$. Without loss of generality, we may assume that $\{i, k\}=\{1,2\}$. Since $1-f_{1} e_{3} \in \operatorname{Spin}_{10}^{+}(q)_{x}$ by Lemma 9.1, $\left\langle e_{\{2,3\}}\right\rangle \cap B \neq \varnothing$ by Lemma 9.3. By a similar argument, $\left\langle e_{\{3,4\}}\right\rangle \cap B \neq \varnothing$. Thus $\delta_{1} e_{\{1,2\}}+\delta_{2} e_{\{3,4\}} \in B$ for some $\delta_{1}, \delta_{2} \in \mathbb{F}_{q}^{*}$, contradicting Lemma 9.2.

Thus $x$ is not a pure spinor, in which case we may assume that $x=1+e_{\{1,2,3,4\}}$ by Lemma 9.2. Let $A:=\{1,2,3,4\}$. Suppose that there exists $T \in I$ such that $\lambda_{T} \neq 0$ and $5 \in T$. Choose $k \in A \backslash T$. Now $T \in I_{5, k}$ and $1-f_{5} e_{k} \in \operatorname{Spin}_{10}^{+}(q)_{x}$ by Lemma 9.1, so Lemma 9.3 implies that $w:=\sum_{S \in I_{5, k}}(-1)^{i_{S}} \lambda_{S} e_{(S \backslash\{5\}) \cup\{k\}} \in B$ for some $i_{S} \in\{0,1\}$. Now $w=\sum_{j \neq 5, k} \mu_{j} e_{\{j, k\}}+\mu e_{A}$ for some $\mu_{j}, \mu \in \mathbb{F}_{q}$. Since $w$ is not pure, $\mu_{j} \neq 0$ for some $j$ (where $j \neq 5, k$ ). Choose $i \in A \backslash\{k, j\}$. Let $\ell^{*}:=j$ and $k^{*}:=i$. Now $1-f_{\ell^{*}} e_{k^{*}} \in \operatorname{Spin}_{10}^{+}(q)_{x}$ by Lemma 9.1, and $\{j, k\}$ is the only element of $I_{\ell^{*}, k^{*}}$ in the decomposition of $w$, so by Lemma $9.3,\left\langle e_{\{i, k\}}\right\rangle \cap B \neq \varnothing$, a contradiction. Thus $5 \notin S$ for all $S \in I$ such that $\lambda_{S} \neq 0$. Suppose that $\lambda_{\{1,2\}} \neq 0$. Now $1-f_{1} e_{3} \in \operatorname{Spin}_{10}^{+}(q)_{x}$ by Lemma 9.1 , so $\left\langle e_{2,3}, e_{3,4}\right\rangle \cap B \neq \varnothing$ by Lemma 9.3, contradicting Lemma 9.2. It follows that $y=\lambda 1+\mu e_{A}$ for some $\lambda, \mu \in \mathbb{F}_{q}^{*}$ such that $\lambda \neq \mu$. Let $u:=e_{2}-f_{4}$ and $v:=f_{3}-e_{1}$, and observe that $s:=1-u v \in \operatorname{Spin}_{10}^{+}(q)$ by Lemma 9.1. Now $1^{s}=1-e_{\{1,2\}}$ and $e_{A}^{s}=e_{A}+e_{\{1,2\}}$, so $x^{s}=x$. Thus $(\mu-\lambda) e_{\{1,2\}}=y^{s}-y \in B$, a contradiction.

We continue to assume that $W=V_{m}(q)$ where $m=2 r \geqslant 8$ and that $Q$ is a non-degenerate form on $W$ of type $\varepsilon=+$ with associated bilinear form $\mathbf{f}$. As above, $C=C(W, Q), \Gamma=\Gamma(C)$, and $W$ has a standard basis $\left\{e_{1}, \ldots, e_{r}, f_{1}, \ldots, f_{r}\right\}$. 
First suppose that $q$ is even, and define $\operatorname{Spin}_{2 r-1}(q):=\left\{s \in \Gamma_{0}^{+}: s^{-1}\left\langle e_{r}+f_{r}\right\rangle s=\left\langle e_{r}+f_{r}\right\rangle\right\}$. By [37, p.197], $\operatorname{Spin}_{2 r-1}(q) \simeq \operatorname{Sp}_{2 r-2}(q)$, and the spin module of $\operatorname{Sp}_{2 r-2}(q)$ is defined to be $C_{+}^{E}$ restricted to $\operatorname{Spin}_{2 r-1}(q)$.

Suppose instead that $q$ is odd. Let $\bar{W}$ be the subspace of $W$ spanned by $e_{r}+f_{r}$ and $e_{1}, \ldots, e_{r-1}, f_{1}, \ldots, f_{r-1}$. Observe that the restriction of $f$ to $\bar{W}$ is non-degenerate. Let $\bar{C}:=$ $C(\bar{W}, Q)$ and $\bar{\Gamma}:=\Gamma(\bar{C})$. We may view $\bar{C}$ as the subalgebra of $C$ generated by $\bar{W}$ by [13, II.1.4], in which case $\bar{C}_{+}=\bar{C} \cap C_{+}$. Further, $\operatorname{Spin}_{2 r-1}(q)=\bar{\Gamma}_{0}^{+}=\bar{\Gamma}^{+} \cap \Gamma_{0}^{+}$by [13, II.6.1]. The spin module of $\operatorname{Spin}_{2 r-1}(q)$ is defined to be the restriction of $C_{+}^{E}$ to $\bar{\Gamma}_{0}^{+}$(see [13, II.5.1 and §3.8]).

Now we consider class $(\mathrm{R} 5)$ where $(m, n, \varepsilon)=(7,8, \circ)$. Note that $\operatorname{Spin}_{7}(q)$ is quasisimple with centre $\operatorname{Spin}_{7}(q) \cap Z=\langle-1\rangle$ where $Z:=Z\left(\mathrm{GL}\left(C_{+}^{E}\right)\right.$ ) since $\operatorname{Sp}_{6}(q)$ ( $q$ even) and $\Omega_{7}(q)$ ( $q$ odd) are simple.

Proposition 9.5. Let $V$ be the spin module of $\operatorname{Spin}_{7}(q)$ where $q$ is a prime power. Let $G$ be an affine permutation group of rank 3 on $V$ where $\operatorname{Spin}_{7}(q) \unlhd G_{0}$. Then there is no $\mathbb{F}_{q}$-independent G-affine proper partial linear space.

Proof. Let $Q$ be a non-degenerate quadratic form on $W:=V_{8}(q)$ with type + and standard basis $\left\{e_{1}, \ldots, e_{4}, f_{1}, \ldots, f_{4}\right\}$. Let $E:=\left\langle e_{1}, \ldots, e_{4}\right\rangle$, so that $V=C_{+}^{E}$, and let $Z:=Z\left(\operatorname{GL}\left(C_{+}^{E}\right)\right)$. Recall that $\operatorname{Spin}_{7}(q) /\left(\operatorname{Spin}_{7}(q) \cap Z\right) \simeq \Omega_{7}(q)$. Recall also that $\operatorname{Spin}_{7}(q) Z$ acts on the set $P$ of pure spinors in $C_{+}^{E}$ and that $|P|=\left(q^{3}+1\right)\left(q^{4}-1\right)$. If $q$ is even, then by [39, Lemma 2.8], $\operatorname{Spin}_{7}(q) Z$ has two orbits on $\left(C_{+}^{E}\right)^{*}$ with sizes $\left(q^{3}+1\right)\left(q^{4}-1\right)$ and $q^{3}(q-1)\left(q^{4}-1\right)$, so $P$ is an orbit of $\operatorname{Spin}_{7}(q) Z$. If $q$ is odd, then by [39, p.494], $\operatorname{Spin}_{7}(q) Z$ has three orbits on $\left(C_{+}^{E}\right)^{*}$, one with size $\left(q^{3}+1\right)\left(q^{4}-1\right)$ and two with size $q^{3}(q-1)\left(q^{4}-1\right) / 2$, so $P$ is an orbit of $\operatorname{Spin}_{7}(q) Z$. Thus one of the orbits of $G_{0}$ is the set of pure spinors. Let $q=p^{e}$ where $p$ is prime.

Suppose for a contradiction that $\left(C_{+}^{E}, \mathcal{L}\right)$ is an $\mathbb{F}_{q}$-independent $G$-affine proper partial linear space. Let $L \in \mathcal{L}_{0}$, let $B:=L^{*}$, and let $x \in B$. By Lemma 2.6, $B$ is a block of $G_{0}$ on $x^{G_{0}}$,

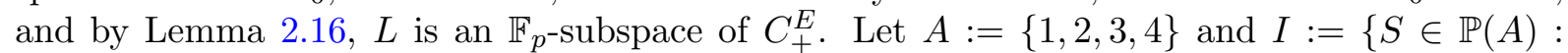
$|S| \equiv 0 \bmod 2\}$. For distinct integers $\ell, k \in A$, let $I_{\ell, k}:=\{S \in I: \ell \in S, k \notin S\}$. Note that $1-f_{\ell} e_{k} \in \operatorname{Spin}_{7}(q)$ for distinct $k, \ell \in\{1,2,3\}$ by Lemma 9.1. By assumption, there exists $y \in B \backslash\langle x\rangle$. Write $y=\sum_{S \in I} \lambda_{S} e_{S}$ where $\lambda_{S} \in \mathbb{F}_{q}$ for $S \in I$.

First suppose that $x=1$. Then $1-f_{\ell} e_{k}$ fixes $x$ for all $k, \ell \in\{1,2,3\}$. We claim that $\left\langle e_{\{i, j\}}\right\rangle \cap B$ is empty for all $i, j \in\{1,2,3\}$ such that $i \neq j$. Suppose otherwise. Then $\lambda e_{\{1,2\}}, \mu e_{\{1,3\}} \in B$ for some $\lambda, \mu \in \mathbb{F}_{q}^{*}$ by Lemma 9.3. Now $s:=1-f_{1}\left(e_{4}+f_{4}\right) \in \operatorname{Spin}_{7}(q)_{x}$ by $\operatorname{Lemma}$ 9.1, so $\mu\left(e_{\{1,3\}}+e_{\{3,4\}}\right)=\left(\mu e_{\{1,3\}}\right)^{s} \in B$. But then $\lambda e_{\{1,2\}}+\mu e_{\{3,4\}} \in B$, contradicting Lemma 9.2. Thus the claim holds. If $\lambda_{T}=0$ for all $T \in I$ such that $|T|=2$, then $y=\lambda_{A} e_{A}$ by Lemma 9.2. However, this implies that $1+\lambda_{A} e_{A} \in B$, contradicting Lemma 9.2. Thus $\lambda_{T} \neq 0$ for some $T \in I$ such that $|T|=2$. Choose $\ell \in T \backslash\{4\}$ and $k \in\{1,2,3\} \backslash T$. By Lemma 9.3, $\lambda e_{\{i, k\}}+\mu e_{\{k, 4\}} \in B$ for some $\lambda, \mu \in \mathbb{F}_{q}$ where $\{i, k, \ell\}=\{1,2,3\}$. If $\lambda \neq 0$, then $\left\langle e_{\{\ell, k\}}\right\rangle \cap B \neq \varnothing$ by Lemma 9.3, a contradiction. Thus $\mu e_{\{k, 4\}} \in B$. Let $s:=1-e_{k} e_{\ell} \in \operatorname{Spin}_{7}(q)$ by Lemma 9.1. Now $s$ fixes $\mu e_{\{k, 4\}}$, so $1^{s} \in B$, but then $e_{\{k, \ell\}} \in B$, a contradiction.

Thus $x$ is not a pure spinor, in which case we may assume that $x=1+e_{A}$ by Lemma 9.2. Suppose that $\lambda_{T} \neq 0$ for some $T \in I$ such that $|T|=2$. Choose $\ell \in T \backslash\{4\}$ and $k \in\{1,2,3\} \backslash T$. Since $x^{1-f_{\ell} e_{k}}=x$, Lemma 9.3 implies that $\left\langle e_{\{i, k\}}, e_{\{k, 4\}}\right\rangle \cap B \neq \varnothing$ where $\{i, k, \ell\}=\{1,2,3\}$, contradicting Lemma 9.2. Thus $y=\lambda 1+\mu e_{A}$ for some $\lambda, \mu \in \mathbb{F}_{q}^{*}$ such that $\lambda \neq \mu$. Let $u:=e_{2}-f_{2}-e_{4}-f_{4}$ and $v:=f_{3}-e_{1}$, and observe that $s:=1-u v \in \operatorname{Spin}_{7}(q)$ by Lemma 9.1. Now $1^{s}=1-e_{\{1,2\}}+e_{\{1,4\}}$ and $e_{A}^{s}=e_{A}+e_{\{1,2\}}-e_{\{1,4\}}$, so $x^{s}=x$. Thus $(\mu-\lambda)\left(e_{\{1,2\}}-e_{\{1,4\}}\right)=$ $y^{s}-y \in B$, contradicting Lemma 9.2.

\section{TEnsor PRODuCT Class (T)}

Let $V:=U \otimes W$ where $U:=V_{2}(q)$ and $W:=V_{m}(q)$ with $q$ a prime power and $m \geqslant 2$. Recall from $\S 2.3$ that for $g \in \mathrm{GL}(U)$ and $h \in \mathrm{GL}(W)$, the linear map $g \otimes h$ maps $u \otimes w$ to 
$u^{g} \otimes w^{h}$ for all $u \in U$ and $w \in W$. Recall also that for $S \leqslant \operatorname{GL}(U)$ and $T \leqslant \operatorname{GL}(W)$, the group $S \otimes T=\{g \otimes h: g \in S, h \in T\} \leqslant \mathrm{GL}(V)$, and $\operatorname{Aut}\left(\mathbb{F}_{q}\right)$ acts naturally on $V, U$ and $W$ in such a way that $(u \otimes w)^{\sigma}=u^{\sigma} \otimes w^{\sigma}$ for all $u \in U, w \in W$ and $\sigma \in \operatorname{Aut}\left(\mathbb{F}_{q}\right)$. Either $m \geqslant 3$ and the stabiliser of the decomposition of $V$ is $(\mathrm{GL}(U) \otimes \operatorname{GL}(W))$ : $\operatorname{Aut}\left(\mathbb{F}_{q}\right)$, or $m=2$, in which case we may assume that $U=W$, and the stabiliser of the decomposition of $V$ is $(\mathrm{GL}(U) \otimes \mathrm{GL}(U)):\left(\operatorname{Aut}\left(\mathbb{F}_{q}\right) \times\langle\tau\rangle\right)$ where $\tau \in \mathrm{GL}(V)$ is defined by $u \otimes w \mapsto w \otimes u$ for all $u, w \in U$. We adopt the convention of writing elements of $(\mathrm{GL}(U) \otimes \mathrm{GL}(W)): \operatorname{Aut}\left(\mathbb{F}_{q}\right)$ as $g \otimes h$ for $g \in \Gamma \mathrm{L}(U)$ and $h \in \Gamma \mathrm{L}(W)$ where $g$ and $h$ are $\sigma$-semilinear for the same $\sigma \in \operatorname{Aut}\left(\mathbb{F}_{q}\right)$. Let $G$ be an affine permutation group of rank 3 on $V$ where $G_{0}$ stabilises the decomposition of $V$. Recall that the orbits of $G_{0}$ on $V^{*}$ are the simple tensors $\left\{u \otimes w: u \in U^{*}, w \in W^{*}\right\}$ and the non-simple tensors $\left\{u_{1} \otimes w_{1}+u_{2} \otimes w_{2}: w_{1}, w_{2} \in W, \operatorname{dim}\left\langle w_{1}, w_{2}\right\rangle=2\right\}$ for any choice of basis $\left\{u_{1}, u_{2}\right\}$ of $U$.

This section is devoted to the proof of the following result, which deals with the classes (T1), (T2) and (T3). However, in the process of proving Proposition 10.1, we will often prove results for more general families of groups in class (T) so that these results can be used for classes (S0), (S1) and (S2).

Proposition 10.1. Let $G$ be an affine permutation group of rank 3 on $V:=U \otimes W$ where $U:=$ $V_{2}(q), W:=V_{m}(q), m \geqslant 2, q$ is a prime power and $G_{0}$ belongs to one of the classes (T1), (T2) or (T3). Then the following are equivalent.

(i) $(V, \mathcal{L})$ is an $\mathbb{F}_{q}$-independent $G$-affine proper partial linear space.

(ii) $\mathcal{L}$ is $\left\{U \otimes w+v: w \in W^{*}, v \in V\right\}$ or $\left\{u \otimes W+v: u \in U^{*}, v \in V\right\}$, and either $m \geqslant 3$, or $m=2$ and $G_{0} \leqslant(\mathrm{GL}(U) \otimes \mathrm{GL}(W)): \operatorname{Aut}\left(\mathbb{F}_{q}\right)$.

Observe that the partial linear spaces of Proposition 10.1(ii) are all described in Example 1.2, though the case $q=2$ is omitted from Proposition 10.1 by our restrictions on $G_{0}$. In Lemma 10.2 below, we prove that the incidence structures $\mathcal{S}_{U}$ and $\mathcal{S}_{W}$ of Example 1.2 are indeed $G$-affine proper partial linear spaces for all $m$ and $q$, where $G:=V:(\mathrm{GL}(U) \otimes \mathrm{GL}(W))$ : Aut $\left(\mathbb{F}_{q}\right)$. We will prove at the end of this section that $G=\operatorname{Aut}\left(\mathcal{S}_{U}\right)=\operatorname{Aut}\left(\mathcal{S}_{W}\right)$.

In order to prove Proposition 10.1, we first consider the orbit of simple tensors.

Lemma 10.2. Let $G$ be an affine permutation group of rank 3 on $V:=U \otimes W$ where $U:=V_{2}(q)$, $W:=V_{m}(q), m \geqslant 2, q$ is a prime power and $G_{0}$ stabilises the decomposition of $V$.

(i) Let $\mathcal{S}:=(V, \mathcal{L})$ be a $G$-affine proper partial linear space in which $\mathcal{S}(0)$ is the set of simple tensors. Let $L \in \mathcal{L}_{0}$ and $u \otimes w \in L^{*}$. Then $L \subseteq U \otimes w$ or $L \subseteq u \otimes W$. Moreover, if $S \otimes T \leqslant G_{0}$ for some $S \leqslant \mathrm{GL}(U)$ and $T \leqslant \mathrm{GL}(W)$, then $L^{*}=C \otimes w$ or $u \otimes C$, where $C$ is a block of $S$ on $U^{*}$ or $T$ on $W^{*}$, respectively.

(ii) Let $H_{0} \leqslant G_{0}$. Then for any $u \in U^{*}$ and $w \in W^{*}$, both $(U \otimes w)^{*}$ and $(u \otimes W)^{*}$ are blocks of $H_{0}$ in its action on the simple tensors unless $m=2$ and $H_{0}$ contains an element interchanging $U$ and $W$, in which case neither are blocks.

(iii) Define $\mathcal{L}$ to be $\left\{U \otimes w+v: w \in W^{*}, v \in V\right\}$ or $\left\{u \otimes W+v: u \in U^{*}, v \in V\right\}$. Then $(V, \mathcal{L})$ is an $\mathbb{F}_{q}$-independent $G$-affine proper partial linear space if and only if either $m \geqslant 3$, or $m=2$ and $G_{0} \leqslant(\mathrm{GL}(U) \otimes \mathrm{GL}(W)): \operatorname{Aut}\left(\mathbb{F}_{q}\right)$.

(iv) If $m=2$, then the two partial linear spaces described in (iii) are isomorphic.

Proof. (i) Suppose that there exists $u_{1} \otimes w_{1} \in L \backslash U \otimes w$ and $u_{2} \otimes w_{2} \in L \backslash u \otimes W$. Then $\left\{w_{1}, w\right\}$ and $\left\{u_{2}, u\right\}$ are both linearly independent sets, and $u_{1} \otimes w_{1}-u \otimes w$ and $u_{2} \otimes w_{2}-u \otimes w$ are both simple by Lemma 2.15(i), so $u_{1}=\lambda u$ and $w_{2}=\mu w$ for some $\lambda, \mu \in \mathbb{F}_{q}^{*}$. Thus $\lambda u \otimes w_{1}-u_{2} \otimes \mu w$ is a non-simple tensor, contradicting Lemma 2.15(i). Hence $L \subseteq U \otimes w$ or $L \subseteq u \otimes W$.

Moreover, suppose that $S \otimes T \leqslant G_{0}$ and $L \subseteq U \otimes w$. Then $L^{*}=C \otimes w$ for some $C \subseteq U^{*}$. Suppose that $v^{\prime}=v^{g}$ for some $v^{\prime}, v \in C$ and $g \in S$. Then $v^{\prime} \otimes w=(v \otimes w)^{g \otimes 1}$, so by Lemma 2.6, $C \otimes w=(C \otimes w)^{g \otimes 1}=C^{g} \otimes w$. Thus $C=C^{g}$. The case where $L \subseteq u \otimes W$ is similar.

(ii) First suppose that $m=2$ and $H_{0}$ contains an element $\nu$ interchanging $U$ and $W$, where we may assume that $W=U$. If $(U \otimes u)^{*}$ is a block for some $u \in U^{*}$, then $(U \otimes u)^{\nu}=u^{\prime} \otimes U$ 
is also a block for some $u^{\prime} \in U^{*}$, but $u^{\prime} \otimes u \in(U \otimes u)^{*} \cap\left(u^{\prime} \otimes U\right)^{*}$, a contradiction. Similarly, $(u \otimes U)^{*}$ is not a block for any $u \in U^{*}$.

Otherwise, $H_{0} \leqslant(\operatorname{GL}(U) \otimes \operatorname{GL}(W))$ : Aut $\left(\mathbb{F}_{q}\right)$. Let $w \in W^{*}$. Suppose that $u^{\prime} \otimes w=(u \otimes w)^{g \otimes h}$ for some $u \otimes w, u^{\prime} \otimes w \in(U \otimes w)^{*}$ and $g \otimes h \in H_{0}$. Then $u^{\prime}=\lambda u^{g}$ and $w=\lambda^{-1} w^{h}$ for some $\lambda \in \mathbb{F}_{q}^{*}$. Hence $(U \otimes w)^{g \otimes h}=U^{g} \otimes \lambda w=U \otimes w$, so $(U \otimes w)^{*}$ is a block. The proof for $(u \otimes W)^{*}$ is similar.

(iii) This follows from (ii) and Lemmas 2.3, 2.6 and 2.7.

(iv) Suppose that $m=2$. Let $\tau \in \operatorname{GL}(V)$ be defined by $u \otimes w \mapsto w \otimes u$ for all $u, w \in U=W$. Now $\tau$ interchanges the line sets of the two partial linear spaces of (iii), so these two partial linear spaces are isomorphic.

Note that in (i), we are not necessarily assuming that $S$ is transitive on $U^{*}$ nor that $T$ is transitive on $W^{*}$. Similarly, we are not necessarily assuming in (ii) that $H_{0}$ is transitive on the set of simple tensors.

Lemma 10.3. Let $G$ be an affine permutation group of rank 3 on $V:=U \otimes W$ where $U:=V_{2}(q)$, $W:=V_{m}(q), m \geqslant 2, q$ is a power of a prime $p$ and $G_{0}$ stabilises the decomposition of $V$. Let $\mathcal{S}:=(V, \mathcal{L})$ be an $\mathbb{F}_{q}$-independent $G$-affine proper partial linear space in which $\mathcal{S}(0)$ is the set of simple tensors. Let $L \in \mathcal{L}_{0}$.

(i) If $\mathrm{SL}(U) \otimes 1 \leqslant G_{0}$ and $L \subseteq U \otimes w$ for some $w \in W^{*}$, then $\mathcal{L}_{0}=\left\{U \otimes v: v \in W^{*}\right\}$.

(ii) If $1 \otimes \mathrm{SL}(W) \leqslant G_{0}$ and $L \subseteq u \otimes W$ for some $u \in U^{*}$, then $\mathcal{L}_{0}=\left\{v \otimes W: v \in U^{*}\right\}$.

(iii) If $q=p$ and $L \subseteq U \otimes w$ for some $w \in W^{*}$, and if $-1 \otimes g \in G_{0}$ for some $g \in \mathrm{GL}(W)_{w}$, then $\mathcal{L}_{0}=\left\{U \otimes v: v \in W^{*}\right\}$.

Proof. If $L=U \otimes w$ or $u \otimes W$, then $\mathcal{L}_{0}=\left\{U \otimes v: v \in W^{*}\right\}$ or $\left\{v \otimes W: v \in U^{*}\right\}$ by Lemmas 2.6 and 10.2(ii). It is routine to verify that if $C$ is a block of $\operatorname{SL}_{n}(q)$ on $V_{n}(q)^{*}$ where $n \geqslant 2$ and $x \in C$, then either $C \subseteq\langle x\rangle_{\mathbb{F}_{q}}$, or $C=V_{n}(q)^{*}$. Thus (i) and (ii) follow from Lemma 10.2(i). For (iii), $L$ is an $\mathbb{F}_{p}$-subspace of $U \otimes w$ by Lemma 2.16, and $|U \otimes w|=p^{2}$, so $L=U \otimes w$.

Lemma 10.4. Let $G$ be an affine permutation group of rank 3 on $V:=U \otimes W$ where $U:=V_{2}(q)$, $W:=V_{m}(q), m \geqslant 2, q$ is a prime power and $G_{0}$ belongs to one of the classes (T1), (T3) or (T4). Let $\mathcal{S}:=(V, \mathcal{L})$ be an $\mathbb{F}_{q}$-independent $G$-affine proper partial linear space in which $\mathcal{S}(0)$ is the set of simple tensors. Then $\mathcal{L}_{0}$ is $\left\{U \otimes w: w \in W^{*}\right\}$ or $\left\{u \otimes W: u \in U^{*}\right\}$.

Proof. Let $L \in \mathcal{L}_{0}$, let $B:=L^{*}$, and let $u \otimes w \in B$. By Lemma 10.2(i), either $L \subseteq U \otimes w$, or $L \subseteq u \otimes W$. Hence we are done when $G_{0}$ lies in (T1) by Lemma 10.3(i)-(ii), so we assume that $G_{0}$ lies in (T3) or (T4). Now $(U \otimes w)^{*}$ and $(u \otimes W)^{*}$ are blocks of $G_{0}$ on $\mathcal{S}(0)$ by Lemma 10.2(ii). If $G_{0}$ lies in (T4), then we use MAGMA to determine that there are exactly two non-trivial blocks of $G_{0}$ on $\mathcal{S}(0)$ that contain $u \otimes w$, so the desired result follows. Suppose that $G_{0}$ lies in (T3), and recall that $q$ is prime and $-1 \otimes g \in G_{0}$ for some $g \in \mathrm{GL}_{m}(q)$. If $L \subseteq u \otimes W$, then $\mathcal{L}_{0}=\left\{v \otimes W: v \in U^{*}\right\}$ by Lemma 10.3(ii). Otherwise, $L \subseteq U \otimes w$. Now there exists $h \in \mathrm{SL}(W)$ such that $\left(w^{g}\right)^{h}=w$, and $-1 \otimes g h \in G_{0}$, so $\mathcal{L}_{0}=\left\{U \otimes v: v \in W^{*}\right\}$ by Lemma 10.3(iii).

Lemma 10.5. Let $G$ be an affine permutation group of rank 3 on $V:=U \otimes W$ where $U:=V_{2}(q)$, $W:=V_{m}(q), m \geqslant 2, q$ is a prime power and $G_{0}$ belongs to $(\mathrm{T} 2)$. Let $\mathcal{S}:=(V, \mathcal{L})$ be an $\mathbb{F}_{q^{-}}$ independent $G$-affine proper partial linear space in which $\mathcal{S}(0)$ is the set of simple tensors. Then $\mathcal{L}_{0}$ is $\left\{U \otimes w: w \in W^{*}\right\}$ or $\left\{u \otimes W: u \in U^{*}\right\}$.

Proof. Let $L \in \mathcal{L}_{0}$ and $B:=L^{*}$. If $L \subseteq u \otimes W$ for some $u \in U^{*}$, then $\mathcal{L}_{0}=\left\{v \otimes W: v \in U^{*}\right\}$ by Lemma 10.3(ii), as desired. Otherwise, by Lemma 10.2(i), $L \subseteq U \otimes w$ for some $w \in W^{*}$, and $B=C \otimes w$, where $C$ is a block of $\mathrm{SL}_{2}(5)$ on $U^{*}$. If $C=U^{*}$, then $\mathcal{L}_{0}=\left\{U \otimes v: v \in W^{*}\right\}$, as desired, so we suppose for a contradiction that $C \neq U^{*}$. Let $U=\left\langle u_{1}, u_{2}\right\rangle$ and $x:=u_{1}$. We may assume that $x \otimes w \in B$, so that $x \in C$. Since $\mathrm{SL}_{2}(5)$ is an irreducible subgroup of $\mathrm{GL}_{2}(q)$, its central involution must be the central involution of $\mathrm{GL}_{2}(q)$. Thus $q=9$ by Lemma 10.3(iii) and the definition of (T2), and $L$ is an $\mathbb{F}_{3}$-subspace of $U \otimes w$ by Lemma 2.16. 
View $\mathrm{SL}_{2}(3)$ as the subgroup of $\mathrm{SL}_{2}(9)$ that acts naturally on the basis $\left\{u_{1}, u_{2}\right\}$ over $\mathbb{F}_{3}$. Assume that $\zeta^{2}=\zeta+1$ where $\zeta:=\zeta_{9}$ (see $\S 2.3$ ). There exists $s \in \mathrm{GL}_{2}(9)$ such that $u_{1}^{s}=\zeta^{2} u_{1}$ and $u_{2}^{s}=-u_{1}+\zeta^{6} u_{2}$. Let $S:=\left\langle\mathrm{SL}_{2}(3), s\right\rangle$. Now $S \simeq \mathrm{SL}_{2}(5)$ (this can be verified using MAGMA), and $\mathrm{GL}_{2}(9)$ has a unique conjugacy class of subgroups isomorphic to $\mathrm{SL}_{2}(5)$, so we may assume that $S \otimes \mathrm{SL}(W) \unlhd G_{0}$ and that $C$ is a block of $S$. The group $S$ has 2 orbits on $U^{*}$, each with size 40, and $S$ acts transitively on the set of 1-spaces of $U$. Let $\Omega_{1}$ and $\Omega_{2}$ be the two orbits of $S$ where $x \in \Omega_{1}$, and note that $\Omega_{2}=\zeta \Omega_{1}$. Since $\left|\mathrm{SL}_{2}(3)_{x}\right|=3$ and $\left|S_{x}\right|=3$, we conclude that $S_{x} \leqslant \mathrm{SL}_{2}(3)$. Then $S_{x}$ has orbits of size 3 on $U \backslash\langle x\rangle$, and no such orbit contains both $u$ and $-u$.

Observe that $|L|$ divides 81 , while $|L|-1=|B|$ divides $\left|(U \otimes w)^{*}\right|=80$ since $(U \otimes w)^{*}$ is a block of $S \otimes \mathrm{SL}(W)$ in its transitive action on the set of simple tensors by Lemma 10.2(ii). Since $\mathcal{S}$ is $\mathbb{F}_{9}$-independent, it follows that $|C|=8$. If $C$ is not contained in $\Omega_{1}$, then by the observations made above concerning the orbits of $S_{x}$, we must have $\left|C \cap \Omega_{2}\right|=6$. However, $C \cap \Omega_{2}$ is a block of $S$ on $\Omega_{2}$, while 6 does not divide 40 , a contradiction. Thus $C \subseteq \Omega_{1}$. In particular, $C=x^{S_{C}}$ and $\left|S_{C}\right|=24$. Recall that $S=\left\langle\mathrm{SL}_{2}(3), s\right\rangle$ where $u_{1}^{s}=\zeta^{2} u_{1}$ and $u_{2}^{s}=-u_{1}+\zeta^{6} u_{2}$. There are exactly two subgroups of $S$ of order 24 that contain $S_{x}$, namely $\mathrm{SL}_{2}(3)$ and $\mathrm{SL}_{2}(3)^{s}$ since $s$ normalises $S_{x}$. Hence $C$ is either $x^{\mathrm{SL}_{2}(3)}=\left\langle u_{1}, u_{2}\right\rangle_{\mathbb{F}_{3}}^{*}$ or

$$
x^{\mathrm{SL}_{2}(3)^{s}}=\left\{ \pm u_{1}\right\} \cup\left\{\zeta^{i} u_{1}+u_{2}: i \in\{3,5,6\}\right\} \cup\left\{\zeta^{j} u_{1}-u_{2}: j \in\{1,2,7\}\right\} .
$$

Let $\lambda:=\zeta^{2}$ and $\mu:=\lambda^{-1}$. There exists $h \in \mathrm{SL}(W)$ such that $w^{h}=\mu w$. Note that $x^{s}=\lambda x$. Now $x \otimes w=(x \otimes w)^{s \otimes h}$, so $C \otimes w=(C \otimes w)^{s \otimes h}=C^{s \mu} \otimes w$. Thus $C=C^{s \mu}$. However, if $C=x^{\mathrm{SL}_{2}(3)}$, then $u_{2} \in C$, so $\zeta^{2} u_{1}-u_{2}=u_{2}^{s \mu} \in C$, a contradiction. Similarly, if $C=x^{\mathrm{SL}_{2}(3)^{s}}$, then $\zeta^{2} u_{1}-u_{2} \in C$, so $u_{2}=\left(\zeta^{2} u_{1}-u_{2}\right)^{s \mu} \in C$, a contradiction.

Next we consider the orbit of non-simple tensors.

Lemma 10.6. Let $G$ be an affine permutation group of rank 3 on $V:=U \otimes W$ where $U:=V_{2}(q)$, $W:=V_{m}(q), m \geqslant 2, q$ is a power of a prime $p, G_{0}$ stabilises the decomposition of $V$ and $1 \otimes \mathrm{SL}(W) \unlhd G_{0}$. Let $\mathcal{S}:=(V, \mathcal{L})$ be a $G$-affine proper partial linear space in which $\mathcal{S}(0)$ is the set of non-simple tensors. Let $L \in \mathcal{L}_{0}$ and $x \in L^{*}$. Write $x=u_{1} \otimes x_{1}+u_{2} \otimes x_{2}$ where $U=\left\langle u_{1}, u_{2}\right\rangle$ and $X:=\left\langle x_{1}, x_{2}\right\rangle \subseteq W$. Then $L$ is an $\mathbb{F}_{p}$-subspace of $U \otimes X$, and $|L|$ divides $q^{2}$.

Proof. Suppose for a contradiction that there exists $y \in L \backslash(U \otimes X)$. Now $y=u_{1} \otimes y_{1}+u_{2} \otimes y_{2}$ for some $y_{1}, y_{2} \in W$, and without loss of generality, we may assume that $y_{1} \notin X$. We may therefore extend $\left\{x_{1}, x_{2}, y_{1}\right\}$ to a basis $\left\{x_{1}, x_{2}, y_{1}, v_{4}, \ldots, v_{m}\right\}$ of $W$. Now there exists $g \in$ $\operatorname{SL}(W)_{x_{1}, x_{2}, v_{4}, \ldots, v_{m}}$ such that $y_{1}^{g}=x_{1}+y_{1}$, in which case $y_{2}^{g}-y_{2}=\lambda x_{1}$ for some $\lambda \in \mathbb{F}_{q}$. By Lemma 2.15, since $x^{1 \otimes g}=x$, it follows that $y^{1 \otimes g} \in L^{*}$ and therefore that the non-zero vector $y^{1 \otimes g}-y=u_{1} \otimes x_{1}+u_{2} \otimes \lambda x_{1}$ is not simple, a contradiction. Thus $L \subseteq u_{1} \otimes X+u_{2} \otimes X=U \otimes X$. There exists $g \in \operatorname{SL}(W)$ such that $x_{1}^{g}=-x_{1}$ and $x_{2}^{g}=-x_{2}$, and $v^{1 \otimes g}=-v$ for all $v \in L$, so $L$ is an $\mathbb{F}_{p}$-subspace of $U \otimes X$ by Lemma 2.16(i). If $u_{1} \otimes w_{1}+u_{2} \otimes w_{2}$ and $u_{1} \otimes w_{1}+u_{2} \otimes w_{3}$ are elements of $L$ for some $w_{1}, w_{2}, w_{3} \in X$, then $u_{2} \otimes\left(w_{3}-w_{2}\right) \in L$, so $w_{2}=w_{3}$. Thus $|L| \leqslant|X|=q^{2}$, and

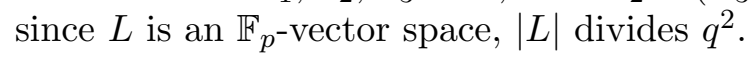

Lemma 10.6 enables us to give a reduction to the case where $m=2$; we include groups satisfying (T5) for our analysis of class (S1) in $\S 11$.

Lemma 10.7. Let $G$ be an affine permutation group of rank 3 on $V:=U \otimes W$ where $U:=$ $V_{2}(q)=\left\langle u_{1}, u_{2}\right\rangle, W:=V_{m}(q)=\left\langle x_{1}, \ldots, x_{m}\right\rangle, m \geqslant 3, q$ is a prime power, $G_{0}$ stabilises the decomposition of $V$ and $1 \otimes \mathrm{SL}(W) \unlhd G_{0}$. Let $X:=\left\langle x_{1}, x_{2}\right\rangle, Y:=U \otimes X$ and $H:=Y: G_{0, Y}^{Y}$. Then $H$ is an affine permutation group of rank 3 on $Y$ such that $H_{0}$ stabilises the decomposition of $Y$ and $1 \otimes \mathrm{GL}(X) \unlhd H_{0}$.

(i) Let $\mathcal{S}:=(V, \mathcal{L})$ be an $\mathbb{F}_{q}$-independent $G$-affine proper partial linear space for which 0 and $u_{1} \otimes x_{1}+u_{2} \otimes x_{2}$ lie on a line L. Then $\mathcal{S} \cap Y=\left(Y, L^{H}\right)$ is an $\mathbb{F}_{q}$-independent $H$-affine proper partial linear space. 
(ii) Let $(Y, \mathcal{L})$ be an $\mathbb{F}_{q}$-independent $H$-affine proper partial linear space for which 0 and $u_{1} \otimes x_{1}+u_{2} \otimes x_{2}$ lie on a line L. Then $\left(V, L^{G}\right)$ is an $\mathbb{F}_{q}$-independent $G$-affine proper partial linear space.

Moreover, if $G_{0}$ belongs to class $(\mathrm{Ti})$ where $i \in\{1,2,3,5\}$, then $H_{0}$ belongs to class $(\mathrm{Ti})$.

Proof. Let $A:=(\mathrm{GL}(U) \otimes \mathrm{GL}(W))$ : Aut $\left(\mathbb{F}_{q}\right)$ where $\operatorname{Aut}\left(\mathbb{F}_{q}\right)$ fixes $x_{1}$ and $x_{2}$. Note that $G_{0} \leqslant A$ since $m \geqslant 3$. Now $A_{Y}=\left(\mathrm{GL}(U) \otimes \mathrm{GL}(W)_{X}\right): \operatorname{Aut}\left(\mathbb{F}_{q}\right)$ and $A_{(Y)}=1 \otimes \mathrm{GL}(W)_{(X)}$, so there is a natural permutation isomorphism between $A_{Y} / A_{(Y)}=A_{Y}^{Y}$ and $(\mathrm{GL}(U) \otimes \mathrm{GL}(X))$ : $\operatorname{Aut}\left(\mathbb{F}_{q}\right)$. In particular, $H_{0}$ stabilises the decomposition of $Y$ and $1 \otimes \mathrm{GL}(X) \simeq(1 \otimes \mathrm{SL}(W))_{Y}^{Y} \unlhd H_{0}$.

Clearly $H$ is an affine permutation group on $Y$. We claim that $H$ has rank 3 on $Y$. Let $Y^{\prime}$ be the set of non-simple tensors of $Y$. Since $1 \otimes \mathrm{GL}(X)$ is faithful and semiregular on $Y^{\prime}$ and $\left|Y^{\prime}\right|=q(q-1)\left(q^{2}-1\right)=|\mathrm{GL}(X)|$, it follows that $1 \otimes \mathrm{GL}(X)$ is regular on $Y^{\prime}$. In particular, $H_{0}$ is transitive on $Y^{\prime}$. Let $u \otimes x, u^{\prime} \otimes x^{\prime}$ be simple tensors in $Y$. There exists $g \otimes h \in G_{0}$ such that $(u \otimes x)^{g \otimes h}=u^{\prime} \otimes x^{\prime}$. Now $u^{g}=\lambda u^{\prime}$ and $x^{h}=\lambda^{-1} x^{\prime}$ for some $\lambda \in \mathbb{F}_{q}^{*}$. Further, there exist $w, w^{\prime} \in X$ such that $X=\langle x, w\rangle=\left\langle x^{\prime}, w^{\prime}\right\rangle$, and since $m \geqslant 3$, there exists $k \in \mathrm{SL}(W)$ such that $\left(x^{\prime}\right)^{k}=x^{\prime}$ and $\left(w^{h}\right)^{k}=w^{\prime}$. Now $g \otimes h k \in G_{0, Y}$ since $X^{h k}=X$, and $(u \otimes x)^{g \otimes h k}=u^{\prime} \otimes x^{\prime}$, so the claim holds.

Suppose that $G_{0}$ belongs to (Ti) where $i \in\{1,2,3,5\}$. If $i=1$ or 2 , then $S \otimes 1 \unlhd G_{0}$ where $S=\mathrm{SL}(U)$ or $\mathrm{SL}_{2}(5)$, respectively, and $S \otimes 1 \simeq(S \otimes 1)_{Y}^{Y} \unlhd H_{0}$, so $H$ belongs to (Ti). If $i=3$, then since $-1 \otimes 1 \in H_{0}$ and $K \otimes \mathrm{GL}(X) \simeq(K \otimes \mathrm{GL}(W))_{Y}^{Y}$ for any $K \leqslant \mathrm{GL}(U)$, it follows that $H$ belongs to (T3). Lastly, if $i=5$, then $H_{0} \leqslant\left(\left(\mathrm{GL}_{1}\left(q^{2}\right) \otimes \operatorname{GL}(W)\right):\left\langle(t \otimes 1) \sigma_{q}\right\rangle\right)_{Y}^{Y} \simeq$ $\left(\mathrm{GL}_{1}\left(q^{2}\right) \otimes \mathrm{GL}(X)\right):\left\langle(t \otimes 1) \sigma_{q}\right\rangle$, so $H$ belongs to (T5).

(i) Lemma 2.13 implies that $\mathcal{S} \cap Y$ is an $H$-affine partial linear space. Since $L \subseteq Y$ by Lemma 10.6, $L$ is also a line of $\mathcal{S} \cap Y$, so $\mathcal{S} \cap Y$ is $\mathbb{F}_{q}$-independent with line-size at least 3, and 0 and $u_{1} \otimes x_{1}$ are non-collinear points, so $\mathcal{S} \cap Y$ is a proper partial linear space. Also, $\mathcal{S} \cap Y=\left(Y, L^{H}\right)$.

(ii) Let $B:=L^{*}$. We claim that $G_{L}$ is transitive on $L$ and that $B$ is a non-trivial block of $G_{0}$ on the non-simple tensors of $V$, for then $\left(V, \mathcal{L}^{G}\right)$ will be a (clearly $\mathbb{F}_{q}$-independent) $G$-affine proper partial linear space by Lemma 2.7. Since $-1 \otimes 1 \in H_{0}$, Lemmas 2.3 and 2.16 imply that $G_{L}$ is transitive on $L$. Suppose that $\left(u_{1} \otimes w_{1}+u_{2} \otimes w_{2}\right)^{g \otimes h}=u_{1} \otimes w_{1}^{\prime}+u_{2} \otimes w_{2}^{\prime}$ for some $u_{1} \otimes w_{1}+u_{2} \otimes w_{2}, u_{1} \otimes w_{1}^{\prime}+u_{2} \otimes w_{2}^{\prime} \in B$ and $g \otimes h \in G_{0}$. Now $X^{h}=\left\langle w_{1}, w_{2}\right\rangle^{h}=\left\langle w_{1}^{\prime}, w_{2}^{\prime}\right\rangle=X$, so $g \otimes h \in G_{0, Y}$. By Lemma 2.6, $B$ is a block of $H_{0}$ on the non-simple tensors of $Y$, so $B^{g \otimes h}=B$. Thus $B$ is a block of $G_{0}$ on the non-simple tensors of $V$, and it is clearly non-trivial.

Lemma 10.8. Let $G$ be an affine permutation group of rank 3 on $V:=U \otimes W$ where $U:=V_{2}(q)$, $W:=V_{m}(q), m \geqslant 2, q$ is a prime power and $G_{0}$ belongs to one of the classes (T1), (T2) or (T3). Then there is no $\mathbb{F}_{q}$-independent $G$-affine proper partial linear space in which 0 is collinear with a non-simple tensor.

Proof. By Lemma 10.7, we may assume that $m=2$. Let $\mathcal{S}:=(V, \mathcal{L})$ be an $\mathbb{F}_{q}$-independent $G$-affine proper partial linear space in which $\mathcal{S}(0)$ is the set of non-simple tensors. Let $q=p^{e}$ where $p$ is prime. Let $L \in \mathcal{L}_{0}$ and $B:=L^{*}$. Now, by Lemma 10.6, $L$ is an $\mathbb{F}_{p}$-subspace of $V$ and $|L|$ divides $q^{2}$. Further, $B$ is a block of $G_{0}$ in its action on the non-simple tensors by Lemma 2.6. Using MAGMA, we determine that there are no such blocks when any of the following cases hold: (T1) with $q=3$, (T2) or (T3). Thus $G_{0}$ belongs to (T1) and $q \geqslant 4$.

We may assume that $U=W$. Now $S:=\mathrm{SL}_{2}(q) \otimes \mathrm{SL}_{2}(q) \unlhd G_{0}$. Let $x:=u_{1} \otimes u_{1}+u_{2} \otimes u_{2} \in B$, where $U=\left\langle u_{1}, u_{2}\right\rangle$. By assumption, there exists $y \in B \backslash\langle x\rangle$, and $y=u_{1} \otimes\left(\lambda_{1} u_{1}+\lambda_{2} u_{2}\right)+$ $u_{2} \otimes\left(\varepsilon_{1} u_{1}+\varepsilon_{2} u_{2}\right)$ for some $\lambda_{1}, \lambda_{2}, \varepsilon_{1}, \varepsilon_{2} \in \mathbb{F}_{q}$. For $g \in \mathrm{SL}_{2}(q)$, let $\tilde{g}$ denote the transpose of $g^{-1}$. Now $S_{x}=\left\{g \otimes \tilde{g}: g \in \mathrm{SL}_{2}(q)\right\}$. Since $q \geqslant 4$, there exists $a \in \mathbb{F}_{q}^{*} \backslash\langle-1\rangle$. There exist $g, h \in \mathrm{SL}_{2}(q)$ such that $u_{1}^{g}=a u_{1}, u_{2}^{g}=a^{-1} u_{2}, u_{1}^{h}=u_{1}$ and $u_{2}^{h}=u_{1}+u_{2}$. Then $u_{1}^{\tilde{g}}=a^{-1} u_{1}$, $u_{2}^{\tilde{g}}=a u_{2}, u_{1}^{\tilde{h}}=u_{1}-u_{2}$ and $u_{2}^{\tilde{h}}=u_{2}$. Since $g \otimes \tilde{g}$ fixes $x$ and $L$ is an $\mathbb{F}_{p^{-}}$subspace of $V$, Lemma 2.15(ii) implies that $z:=y^{g \otimes \tilde{g}}-y \in L$. Let $\lambda:=\left(a^{2}-1\right) \lambda_{2}$ and $\varepsilon:=\left(a^{-2}-1\right) \varepsilon_{1}$. 
Then $z=u_{1} \otimes \lambda u_{2}+u_{2} \otimes \varepsilon u_{1}$. Suppose that $\varepsilon=0$. Then $\lambda=0$ since $B$ only contains nonsimple tensors. Since $a \notin\langle-1\rangle$, it follows that $\lambda_{2}=\varepsilon_{1}=0$, so $y=\lambda_{1} u_{1} \otimes u_{1}+\varepsilon_{2} u_{2} \otimes u_{2}$. Now $\left(\varepsilon_{2}-\lambda_{1}\right) u_{1} \otimes u_{2}=y^{h \otimes \tilde{h}}-y \in L$ since $h \otimes \tilde{h}$ fixes $x$, so $\varepsilon_{2}=\lambda_{1}$, but then $y \in\langle x\rangle$, a contradiction. Thus $\varepsilon \neq 0$. Let $w:=u_{1} \otimes \varepsilon\left(u_{1}-u_{2}\right)+u_{2} \otimes(-\varepsilon) u_{2}=z^{h \otimes \tilde{h}}-z \in B$. Then $u_{1} \otimes\left(1-a^{2}\right) \varepsilon u_{2}=w^{g \otimes \tilde{g}}-w \in B$, a contradiction.

Proof of Proposition 10.1. If (i) holds, then 0 is collinear with a simple tensor by Lemma 10.8, so (ii) holds by Lemmas 10.4, 10.5 and 10.2(iii). Conversely, if (ii) holds, then (i) holds by Lemma 10.2(iii).

To finish this section, we prove a result that enables us to determine the full automorphism group of a $G$-affine proper partial linear space when $G$ belongs to class (T). We then apply this result to the partial linear spaces of Example 1.2.

Lemma 10.9. Let $G$ be an affine permutation group of rank 3 on $V:=U \otimes W$ where $U:=V_{2}(q)$, $W:=V_{m}(q), m \geqslant 2, q$ is a prime power and $G_{0}$ stabilises the decomposition of $V$. Let $\mathcal{S}$ be a $G$-affine proper partial linear space. Then $\operatorname{Aut}(\mathcal{S})$ is an affine permutation group on $V$ and $\operatorname{Aut}(\mathcal{S})_{0}$ stabilises the decomposition of $V$.

Proof. Let $\Gamma$ be the graph with vertex set $V$ in which distinct vectors $v$ and $v^{\prime}$ are adjacent if and only if $v-v^{\prime}$ is a simple tensor. Then $\Gamma$ is isomorphic to the bilinear forms graph $H_{q}(2, m)$ $($ see $[8, \S 9.5 \mathrm{~A}])$. By $\left[8\right.$, Theorem 9.5.1], $\operatorname{Aut}(\Gamma)=V$ : $\operatorname{Aut}(\Gamma)_{0}$, where $\operatorname{Aut}(\Gamma)_{0}$ is the full stabiliser of the decomposition of $V$. By Lemma 2.4 and Remark 2.5, the collinearity graph of $\mathcal{S}$ is either $\Gamma$ or the complement of $\Gamma$, so $\operatorname{Aut}(\mathcal{S}) \leqslant \operatorname{Aut}(\Gamma)$, and the result holds since $V \leqslant G \leqslant \operatorname{Aut}(\mathcal{S})$.

Proposition 10.10. Let $V:=U \otimes W$ where $U:=V_{2}(q), W:=V_{m}(q), m \geqslant 2$, and $q$ is a prime power. Let $\mathcal{S}:=(V, \mathcal{L})$ where $\mathcal{L}$ is $\left\{(U \otimes w)+v: w \in W^{*}, v \in V\right\}$ or $\left\{(u \otimes W)+v: u \in U^{*}, v \in V\right\}$. Then $\operatorname{Aut}(\mathcal{S})=V:\left(\mathrm{GL}_{2}(q) \otimes \mathrm{GL}_{m}(q)\right): \operatorname{Aut}\left(\mathbb{F}_{q}\right) \simeq V:\left(\mathrm{GL}_{2}(q) \circ \mathrm{GL}_{m}(q)\right): \operatorname{Aut}\left(\mathbb{F}_{q}\right)$.

Proof. This follows from Lemmas 10.2(iii) and 10.9.

\section{Subfield Classes (S1) and (S2)}

Classes (S1) and (S2) consist of those affine permutation groups $G$ of rank 3 on $V_{n}\left(q^{2}\right)$ for which either $\mathrm{SL}_{n}(q) \unlhd G_{0}$ where $n \geqslant 2$, or $A_{7} \unlhd G_{0}$ where $(n, q)=(4,2)$ and $A_{7} \leqslant \mathrm{SL}_{4}(2) \simeq A_{8}$. Let $r:=q^{2}, K:=\mathbb{F}_{r}$ and $\zeta:=\zeta_{r}$ (see $\left.\S 2.3\right)$. Let $\left\{v_{1}, \ldots, v_{n}\right\}$ be a basis of $V_{n}(r)$ on which $\mathrm{GL}_{n}(q)$ acts naturally over $\mathbb{F}_{q}$, and let $W:=\left\langle v_{1}, \ldots, v_{n}\right\rangle_{\mathbb{F}_{q}}$. By assumption, $G_{0} \leqslant \Gamma L_{n}(r)$, so $G_{0} \leqslant\left(\mathrm{GL}_{n}(q) \circ K^{*}\right):\left\langle\sigma_{r}\right\rangle$ where $\sigma_{r}$ (see $\left.\S 2.3\right)$ acts on $V_{n}(r)$ with respect to $\left\{v_{1}, \ldots, v_{n}\right\}$. Representatives for the orbits of $G_{0}$ are $v_{1}$ and $v_{1}+\zeta v_{2}$, and $v_{1}^{G_{0}}=\left\{\lambda v: \lambda \in K^{*}, v \in W^{*}\right\}$. Let $\lambda W:=\{\lambda v: v \in W\}$ for $\lambda \in K^{*}$. The orbits of $\mathrm{SL}_{n}(q)$ on $v_{1}^{G_{0}}$ are $\left(\lambda_{1} W\right)^{*}, \ldots,\left(\lambda_{s} W\right)^{*}$, where $\lambda_{1}, \ldots, \lambda_{s}$ is a transversal for $\mathbb{F}_{q}^{*}$ in $K^{*}$ (so $s=q+1$ ).

Here is an equivalent definition for the classes (S1) and (S2). As above, let $r:=q^{2}, K:=\mathbb{F}_{r}$ and $\zeta:=\zeta_{r}$, and let $\left\{v_{1}, \ldots, v_{n}\right\}$ be a basis of $W:=V_{n}(q)$. The extension of scalars $K \otimes W$ is an $n$-dimensional $K$-vector space with basis $\left\{1 \otimes v_{1}, \ldots, 1 \otimes v_{n}\right\}$ on which $\left(\mathrm{GL}_{1}(r) \otimes \mathrm{GL}_{n}(q)\right):\left\langle\sigma_{r}\right\rangle$ acts naturally. Now classes (S1) and (S2) consist of those affine permutation groups $G$ of rank 3 on $K \otimes W$ for which either $1 \otimes \mathrm{SL}_{n}(q) \unlhd G_{0}$ where $n \geqslant 2$, or $1 \otimes A_{7} \unlhd G_{0}$ where $(n, q)=(4,2)$ and $A_{7} \leqslant \mathrm{SL}_{4}(2)$. Representatives for the orbits of $G_{0}$ are $1 \otimes v_{1}$ and $1 \otimes v_{1}+\zeta \otimes v_{2}$. Further, we may view $K \otimes W$ as a $2 n$-dimensional $\mathbb{F}_{q}$-vector space with basis $\left\{1 \otimes v_{1}, \ldots, 1 \otimes v_{n}, \zeta \otimes v_{1}, \ldots, \zeta \otimes v_{n}\right\}$, in which case $G_{0}$ lies in class (T5) or (T4), respectively. (Note that in class (T5), there exists $t \in \mathrm{GL}_{2}(q)$ such that $t \sigma_{q}$ acts as the Frobenius automorphism of $K$.)

The orbits of $1 \otimes \operatorname{SL}_{n}(q)$ on $\left(1 \otimes v_{1}\right)^{G_{0}}$ are $\lambda_{1} \otimes W^{*}, \ldots, \lambda_{s} \otimes W^{*}$ where $\lambda_{1}, \ldots, \lambda_{s}$ is a transversal for $\mathbb{F}_{q}^{*}$ in $K^{*}$. If we define $\mathcal{L}$ to be the set of translations of $\lambda_{i} \otimes W$ for $1 \leqslant i \leqslant q+1$, then $(K \otimes W, \mathcal{L})$ is one of the proper partial linear spaces described in Example 1.2 and Lemma 10.2(iii), and it is both $K$-independent and $\mathbb{F}_{q}$-independent. However, if we instead define $\mathcal{L}$ to be the set of translations of $K \otimes v=\langle 1 \otimes v\rangle_{K}$ for $v \in W^{*}$, then $(K \otimes W, \mathcal{L})$ is not only the other proper partial 
linear space described in Example 1.2 and Lemma 10.2(iii), but also a partial linear space from Example 1.1 that is $K$-dependent and $\mathbb{F}_{q}$-independent. (In the notation of Example 1.1, for any $G_{0}$ in class (S1) or (S2), the partial linear space is $\mathcal{S}_{1}$, where $\Delta_{1}$ is the orbit $\left\{\langle 1 \otimes v\rangle_{K}: v \in W^{*}\right\}$ of $G_{0}$ on the points of $\mathrm{PG}_{n-1}\left(q^{2}\right)$.)

By combining these two viewpoints of classes (S1) and (S2), we obtain the following as an immediate consequence of Lemmas 10.2, 10.3(ii) and Lemma 10.4.

Proposition 11.1. Let $G$ be an affine permutation group of rank 3 on $V:=V_{n}\left(q^{2}\right)$ where $q$ is a prime power and either $\mathrm{SL}_{n}(q) \unlhd G_{0}$ and $n \geqslant 2$, or $A_{7} \unlhd G_{0}$ and $(n, q)=(4,2)$. Let $K:=\mathbb{F}_{q^{2}}$. Let $\left\{v_{1}, \ldots, v_{n}\right\}$ be a basis of $V$ on which $\mathrm{GL}_{n}(q)$ acts naturally over $\mathbb{F}_{q}$, and let $W:=\left\langle v_{1}, \ldots, v_{n}\right\rangle_{\mathbb{F}_{q}}$.

(i) The only $K$-independent $G$-affine proper partial linear space for which 0 and $v_{1}$ are collinear has line set $\mathcal{L}:=\left\{\lambda W+v: \lambda \in K^{*}, v \in V\right\}$.

(ii) The partial linear space $(V, \mathcal{L})$ is described in Example 1.2 .

Next we consider the orbit containing $v_{1}+\zeta v_{2}$.

Proposition 11.2. Let $G$ be an affine permutation group of rank 3 on $V:=V_{n}\left(q^{2}\right)$ where $q$ is a prime power and either $\mathrm{SL}_{n}(q) \unlhd G_{0}$ and $n \geqslant 2$, or $A_{7} \unlhd G_{0}$ and $(n, q)=(4,2)$. Let $r:=q^{2}$, $K:=\mathbb{F}_{r}$ and $\zeta:=\zeta_{r}$ (where $\zeta^{2}=\zeta+1$ when $q=3$ ). Let $\left\{v_{1}, \ldots, v_{n}\right\}$ be a basis of $V$ on which $\mathrm{GL}_{n}(q)$ acts naturally over $\mathbb{F}_{q}$. Let $x:=v_{1}+\zeta v_{2}, y_{1}:=\zeta^{2} v_{1}+v_{2}$ and $y_{2}:=-\zeta v_{1}+v_{2}$.

(i) Let $A$ be the affine permutation group on $V$ for which $A_{0}=\mathrm{GL}_{n}(q)\left\langle\zeta^{2}, \zeta \sigma_{r}\right\rangle$, where $\sigma_{r}$ acts on $V$ with respect to $\left\{v_{1}, \ldots, v_{n}\right\}$. Then $A$ has rank 3 .

(ii) $(V, \mathcal{L})$ is a $K$-independent $G$-affine proper partial linear space for which 0 and $x$ are collinear if and only if $q=3, G \leqslant A$ and $\mathcal{L}=L^{A}$ where $L=\left\langle x, y_{1}\right\rangle_{\mathbb{F}_{3}}$ or $\left\langle x, y_{2}\right\rangle_{\mathbb{F}_{3}}$.

(iii) Let $q:=3$. Let $L_{3}:=\langle x\rangle_{K}$ and $L_{i}:=\left\langle x, y_{i}\right\rangle_{\mathbb{F}_{3}}$ for $i=1,2$. Let $\mathcal{S}_{i}$ be the partial linear space $\left(V, L_{i}^{A}\right)$ for $1 \leqslant i \leqslant 3$. Then $\mathcal{S}_{3}$ is $K$-dependent and $\mathcal{S}_{1} \simeq \mathcal{S}_{2} \simeq \mathcal{S}_{3}$.

When $\mathrm{SL}_{n}(q) \unlhd G_{0}$, we will use Lemma 10.7 to reduce to the case $n=2$. In order to deal with the case $n=2$, we require the following technical result.

Lemma 11.3. Let $q:=p^{e}$ where $p$ is a prime and $e \geqslant 1$, and let $s:=p^{f}$ where $1 \leqslant f<2 e$ and $f$ divides $2 e$. If $(q+1) / \operatorname{gcd}(q+1,4 e)$ divides $s-1$, then $(q, s)$ is $(3,3),(7,7)$ or $(8,4)$.

Proof. Since $f$ is a proper divisor of $2 e$, it follows that $f \leqslant e$. First suppose that $f=e$. Then $s=q$, so $q+1=a \operatorname{gcd}(q+1,4 e)$ for some $a \in\{1,2\}$, in which case $q=3$ or 7 .

Now suppose that $f<e$. Since $f$ divides $2 e$, it follows that $f \leqslant 2 e / 3$. Define $m$ to be 1 when $q$ is even, 2 when $q \equiv 1 \bmod 4$, and 4 when $q \equiv 3 \bmod 4$. Now $p^{e} / m e<p^{2 e / 3}$, so $p^{e}<(m e)^{3}$.

If $q$ is even, then $2 \leqslant e \leqslant 9$. Since $(q+1) / \operatorname{gcd}(q+1, e) \leqslant p^{2 e / 3}-1$, it follows that $q$ is $2^{3}$ or $2^{9}$. Since $(q+1) / \operatorname{gcd}(q+1, e)$ divides $s-1$ and $f \mid 2 e$ but $f<e$, it follows that $(q, s)=(8,4)$.

If $q$ is odd, then $2 \leqslant e \leqslant 10$, so $p \leqslant 19$. Since $(q+1) / \operatorname{gcd}(q+1,4 e) \leqslant p^{2 e / 3}-1$, it follows that $q$ is $3^{3}, 5^{3}$, or $11^{3}$. Then $f \in\{1,2\}$, but $(q+1) / \operatorname{gcd}(q+1,4 e) \nmid(s-1)$.

Now we consider the case $n=2$.

Proposition 11.4. Let $G$ be an affine permutation group of rank 3 on $V:=V_{2}\left(q^{2}\right)$ where $\mathrm{SL}_{2}(q) \unlhd G_{0}$ and $q$ is a prime power. Let $r:=q^{2}, K:=\mathbb{F}_{r}$ and $\zeta:=\zeta_{r}$ (where $\zeta^{2}=\zeta+1$ when $q=3)$. Let $\left\{v_{1}, v_{2}\right\}$ be a basis of $V$ on which $\mathrm{GL}_{2}(q)$ acts naturally over $\mathbb{F}_{q}$. Let $A$ be the affine permutation group on $V$ for which $A_{0}=\mathrm{GL}_{2}(q)\left\langle\zeta^{2}, \zeta \sigma_{r}\right\rangle$, where $\sigma_{r}$ acts on $V$ with respect to $\left\{v_{1}, v_{2}\right\}$. The following are equivalent.

(i) $(V, \mathcal{L})$ is a $K$-independent $G$-affine proper partial linear space for which 0 and $v_{1}+\zeta v_{2}$ are collinear.

(ii) $q=3, G \leqslant A$ and $\mathcal{L}=L^{A}$ where $L=\left\langle v_{1}+\zeta v_{2}, \zeta^{2} v_{1}+v_{2}\right\rangle_{\mathbb{F}_{3}}$ or $\left\langle v_{1}+\zeta v_{2},-\zeta v_{1}+v_{2}\right\rangle_{\mathbb{F}_{3}}$.

Proof. If (ii) holds, then (i) holds by Lemmas 2.3 and 2.7 and a computation in Magma.

Conversely, suppose that (i) holds, and let $\mathcal{S}:=(V, \mathcal{L})$. By Lemmas 10.6 and 4.3, there exists a subfield $\mathbb{F}_{s}$ of $K$ such that each $L \in \mathcal{L}_{0}$ is an $\mathbb{F}_{s}$-subspace of $V$ with the property that 
$\mathbb{F}_{s}=\{\lambda \in K: \lambda u \in L\}$ for all $u \in L^{*}$. Write $q=p^{e}$ and $s=p^{f}$ where $p$ is prime. By Lemma 10.6, $\mathcal{S}$ has line-size at most $q^{2}$. Since $\mathcal{S}$ is $K$-independent, $\mathbb{F}_{s}$ is a proper subfield of $K$, so $f$ is a proper divisor of $2 e$. If $q=3$, then (ii) holds by Lemma 2.6 and a computation in Magma, so we assume that $q \neq 3$. We claim that $(q+1) / \operatorname{gcd}(q+1,4 e)$ divides $s-1$. If so, then $(q, s)$ is $(7,7)$ or $(8,4)$ by Lemma 11.3 , in which case we use MAGMA and Lemma 2.6(i) and (ii) to obtain a contradiction.

Since $\mathrm{SL}_{2}(q)$ and $\mathrm{SU}_{2}(q)$ are conjugate in $\mathrm{GL}(V)$ (see case (2) of the proof of Theorem 3.1), we may assume that $\mathrm{SU}_{2}(q) \unlhd G_{0} \leqslant \Gamma \mathrm{U}_{2}(q)$, in which case the orbits of $G_{0}$ on $V^{*}$ consist of the nonzero isotropic vectors and the non-isotropic vectors, with sizes $\left(q^{2}-1\right)(q+1)$ and $q(q-1)\left(q^{2}-1\right)$, respectively. Further, the vector $v_{1}+\zeta v_{2}$ in (i) corresponds to a non-isotropic vector, so 0 is collinear with a non-isotropic vector. Let $\mathbf{f}$ be the non-degenerate unitary form preserved by $\mathrm{SU}_{2}(q)$, and let $\left\{u_{1}, u_{2}\right\}$ be an orthonormal basis for $V$ (see [37, Proposition 2.3.1]). Then $u_{1}$ is non-isotropic. Let $N$ be the kernel of the (multiplicative) norm map $\lambda \mapsto \lambda^{q+1}$ for $\lambda \in K^{*}$. Let $D$ be the set of $g \in \mathrm{GL}_{2}\left(q^{2}\right)$ such that $u_{1}^{g}=u_{1}$ and $u_{2}^{g}=\lambda u_{2}$ for some $\lambda \in N$, and observe that $D \simeq C_{q+1}$ since the norm map is surjective. It is routine to verify that $\Gamma \mathrm{U}_{2}(q)_{u_{1}} \cap \mathrm{GL}_{2}\left(q^{2}\right)=D$, so $\Gamma \mathrm{U}_{2}(q)_{u_{1}} / D$ is a group of order $2 e$, and $\left|G_{0, u_{1}} /\left(G_{0, u_{1}} \cap D\right)\right|$ divides $2 e$.

Since the subdegrees of $G$ are $\left(q^{2}-1\right)(q+1)$ and $\left|u_{1}^{G_{0}}\right|=q(q-1)\left(q^{2}-1\right)$, it follows that the least common multiple of $q+1$ and $q-1$ divides $\left|G_{0}\right| / q\left(q^{2}-1\right)=(q-1)\left|G_{0, u_{1}}\right|$. Thus $m:=(q+1) / \operatorname{gcd}(q+1, q-1)$ divides $\left|G_{0, u_{1}}\right|$, which divides $\left|G_{0, u_{1}} \cap D\right| 2 e$, so $m / \operatorname{gcd}(m, 2 e)$ divides $\left|G_{0, u_{1}} \cap D\right|$. Now $\operatorname{gcd}(q+1, q-1)=\operatorname{gcd}(2, q-1)$, so $\left|G_{0, u_{1}} \cap D\right|$ is divisible by $(q+$ $1) / \operatorname{gcd}(q+1,4 e)$. Thus, for any non-isotropic vector $v \in V$, the order of $\left|G_{0, v} \cap \mathrm{GL}_{2}\left(q^{2}\right)\right|$ is divisible by $(q+1) / \operatorname{gcd}(q+1,4 e)$. Moreover, $(q+1) \neq \operatorname{gcd}(q+1,4 e)$ since $q \neq 3$, so there exists $h \in G_{0, u_{1}} \cap D$ such that $h \neq 1$, in which case $u_{2}^{h}=\lambda u_{2}$ for some $\lambda \in N \backslash\{1\}$.

Let $L$ be the line of $\mathcal{S}$ on 0 and $u_{1}$, and let $B:=L^{*}$. By Lemma 2.6, $B$ is a block of $G_{0}$ on the set of non-isotropic vectors. By assumption, there exists $y \in B \backslash\left\langle u_{1}\right\rangle$. Write $y=\lambda_{1} u_{1}+\lambda_{2} u_{2}$ where $\lambda_{1}, \lambda_{2} \in K$. Now $\lambda_{1} u_{1}+\lambda \lambda_{2} u_{2}=y^{h} \in B$ by Lemma $2.15(\mathrm{ii})$, so $\delta u_{2} \in B$ where $\delta:=(\lambda-1) \lambda_{2}$

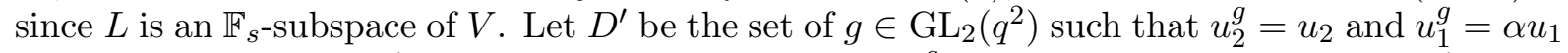
for some $\alpha \in N$. Let $N^{\prime}$ be the set of $\alpha \in N$ such that $u_{1}^{g}=\alpha u_{1}$ for some $g \in G_{0, \delta u_{2}} \cap D^{\prime}$. Then $N^{\prime} \leqslant N$, and $N^{\prime} \simeq G_{0, \delta u_{2}} \cap D^{\prime}=G_{0, \delta u_{2}} \cap \mathrm{GL}_{2}\left(q^{2}\right)$, so $(q+1) / \operatorname{gcd}(q+1,4 e)$ divides $\left|N^{\prime}\right|$. If $\mu \in N^{\prime}$, then $\mu u_{1} \in B$ by Lemma 2.15(ii), so $\mu \in \mathbb{F}_{s}^{*}$. Thus $N^{\prime} \leqslant \mathbb{F}_{s}^{*}$, and the claim follows.

Proof of Proposition 11.2. (i) This is routine.

(ii) If $A_{7} \unlhd G_{0}$ and $(n, q)=(4,2)$, then there are no $K$-independent $G$-affine proper partial linear spaces for which 0 and $x$ are collinear by Lemmas 2.6 and 2.16 and a computation in MAGMA, so we assume that $\mathrm{SL}_{n}(q) \unlhd G_{0}$. If $n=2$, then we are done by Proposition 11.4, so we assume that $n \geqslant 3$. Let $Y:=\left\langle v_{1}, v_{2}\right\rangle_{K}$ and $H:=W: G_{0, Y}^{Y}$. By Lemma 10.7, $\mathrm{GL}_{2}(q) \unlhd H_{0}$ and $H$ is transitive of rank 3 .

First we claim that if $q=3$ and $H_{0} \leqslant \mathrm{GL}_{2}(3)\left\langle\zeta^{2}, \zeta \sigma_{9}\right\rangle$, then $H_{0}=\mathrm{GL}_{2}(3)\left\langle\zeta^{2}, \zeta \sigma_{9}\right\rangle$. Since $H$ has rank 3, there exists $g \in H_{0}$ mapping $v_{1}$ to $\zeta^{2} v_{1}$, and $g=h \zeta^{2 i}\left(\zeta \sigma_{9}\right)^{j}$ for some $h \in \mathrm{GL}_{2}(3)$, $i \in\{0,1\}$ and $j \in\{0,1\}$, but this is impossible if $j=1$, so $j=0$, whence $i=1$. Since $\mathrm{GL}_{2}(3) \leqslant H_{0}$, it follows that $\zeta^{2} \in H_{0}$. Similarly, there exists $g \in H_{0}$ mapping $v_{1}$ to $\zeta v_{1}$, so $\zeta \sigma_{9} \in H_{0}$, and the claim holds.

Let $\mathcal{S}:=(V, \mathcal{L})$ be a $K$-independent $G$-affine proper partial linear space for which 0 and $x$ lie on a line $L$. Then $\mathcal{S}$ is $\mathbb{F}_{q^{-}}$-independent, so by Lemma $10.7(\mathrm{i}), \mathcal{S} \cap Y=\left(Y, L^{H}\right)$ is an $H$ affine proper partial linear space. Since $\mathcal{S} \cap Y$ contains the line $L$, it is $K$-independent, so by Proposition 11.4 and the claim, $q=3, H_{0}=\mathrm{GL}_{2}(3)\left\langle\zeta^{2}, \zeta \sigma_{9}\right\rangle$ and $L=\left\langle x, y_{1}\right\rangle_{\mathbb{F}_{3}}$ or $\left\langle x, y_{2}\right\rangle_{\mathbb{F}_{3}}$. Let $g \in G_{0}$. Then $Y^{g}=Y^{h}$ for some $h \in \mathrm{GL}_{n}(3)$. There exists $s \in \mathrm{SL}_{n}(3)$ such that $v_{1}^{h s}=v_{1}$ and $v_{2}^{h s}=v_{2}$, so $g s \in G_{0, Y} \leqslant A_{0}$. Thus $G \leqslant A$. Further, $L^{G}=L^{A}$ by Lemma 10.7(ii) since $A$ is a rank 3 group and $A_{0, Y}^{Y}=H_{0}$. 
Conversely, suppose that $q=3, G \leqslant A$ and $\mathcal{L}=L^{A}$ where $L=\left\langle x, y_{1}\right\rangle_{\mathbb{F}_{3}}$ or $\left\langle x, y_{2}\right\rangle_{\mathbb{F}_{3}}$. Then $H_{0} \leqslant \mathrm{GL}_{2}(3)\left\langle\zeta^{2}, \zeta \sigma_{9}\right\rangle$, so $H_{0}=\mathrm{GL}_{2}(3)\left\langle\zeta^{2}, \zeta \sigma_{9}\right\rangle$ by the claim. Thus $(V, \mathcal{L})$ is a $G$-affine proper partial linear space by Lemma 10.7 (ii) and Proposition 11.4, and it is clearly $K$-independent.

(iii) Clearly $\mathcal{S}_{3}$ is $K$-dependent. Since $\zeta$ normalises $A$, it follows that $\zeta$ maps $\mathcal{L}_{1}$ to $\mathcal{L}_{2}$, so it suffices to show that $\mathcal{S}_{2}$ and $\mathcal{S}_{3}$ are isomorphic. Since $\left\{v_{1}, \ldots, v_{n}, \zeta v_{1}, \ldots, \zeta v_{n}\right\}$ is an $\mathbb{F}_{3}$-basis of $V$, there exists $t \in \mathrm{GL}_{2 n}(3)$ such that $v_{i}^{t}=\zeta^{2} v_{i}$ and $\left(\zeta v_{i}\right)^{t}=-v_{i}$ for $1 \leqslant i \leqslant n$. Now $t$ centralises $\mathrm{GL}_{n}(3)$, and $t^{-1} \zeta \sigma_{9} t=\zeta^{3} \sigma_{9}$ and $t^{-1} \zeta^{2} t=\zeta \sigma_{9}$, so $t$ normalises $A_{0}$. Further, $x^{t}=\zeta^{2} v_{1}-v_{2}$ and $y_{2}^{t}=v_{1}+\zeta^{2} v_{2}$, so $y_{2}^{t}=\zeta^{6} x^{t}$, in which case $L_{2}^{t}=\left\langle x^{t}\right\rangle_{K}$. Thus $\left(L_{2}^{A}\right)^{t}=\left(\left\langle x^{t}\right\rangle_{K}\right)^{A}=L_{3}^{A}$.

In Remark 2.12, we observed that if $\mathcal{S}:=(\mathcal{P}, \mathcal{L})$ and $\mathcal{S}^{\prime}:=\left(\mathcal{P}^{\prime}, \mathcal{L}^{\prime}\right)$ are isomorphic linear spaces satisfying the conditions of Lemma 2.11(i), then any isomorphism $\varphi: \mathcal{S} \rightarrow \mathcal{S}^{\prime}$ determines isomorphisms between the proper partial linear spaces obtained from $\mathcal{S}$ and $\mathcal{S}^{\prime}$ (in the sense of Lemma 2.11). In the following, we show that the converse of this observation need not hold.

Remark 11.5. Let $V:=V_{2}\left(q^{2}\right)$ where $q$ is a prime power, let $K:=\mathbb{F}_{r}$ where $r:=q^{2}$, and let $G$ be the rank 3 affine primitive permutation group $V:\left(\mathrm{GL}_{2}(q) \circ K^{*}\right)$ : $\operatorname{Aut}(K)$ from class $(\mathrm{S} 1)$. Let $\left\{v_{1}, v_{2}\right\}$ be a basis of $V$ on which $\mathrm{GL}_{2}(q)$ acts naturally over $\mathbb{F}_{q}$, and let $W:=\left\langle v_{1}, v_{2}\right\rangle_{\mathbb{F}_{q}}$ and $Y:=\left(v_{1}+\zeta_{r} v_{2}\right)^{G_{0}}$. Let $\mathcal{L}_{U}:=\left\{\langle w\rangle_{K}+v: w \in W^{*}, v \in V\right\}, \mathcal{L}_{W}:=\left\{\lambda W+v: \lambda \in K^{*}, v \in V\right\}$ and $\mathcal{L}_{Y}:=\left\{\langle w\rangle_{K}+v: w \in Y, v \in V\right\}$. Let $\mathcal{S}_{U}:=\left(V, \mathcal{L}_{U}\right), \mathcal{S}_{W}:=\left(V, \mathcal{L}_{W}\right)$ and $\mathcal{S}_{Y}:=\left(V, \mathcal{L}_{Y}\right)$. Now $\mathcal{S}_{U}, \mathcal{S}_{W}$ and $\mathcal{S}_{Y}$ are $G$-affine proper partial linear spaces, and the collinearity relation of $\mathcal{S}_{U}$ and $\mathcal{S}_{W}$ is disjoint from the collinearity relation of $\mathcal{S}_{Y}$. Further, as we discussed at the beginning of this section, $\mathcal{S}_{U}$ and $\mathcal{S}_{W}$ are the two partial linear spaces of Example 1.2 (for $m=2$ ). Thus $\mathcal{S}_{U} \simeq \mathcal{S}_{W}$ by Lemma 10.2(iv), and of course $\mathcal{S}_{Y}$ is isomorphic to itself. However, the linear spaces $\mathcal{S}_{1}:=\left(V, \mathcal{L}_{U} \cup \mathcal{L}_{Y}\right)$ and $\mathcal{S}_{2}:=\left(V, \mathcal{L}_{W} \cup \mathcal{L}_{Y}\right)$ (see Lemma 2.11) are not isomorphic for $q \geqslant 3: \mathcal{S}_{1}$ is the affine plane $\mathrm{AG}_{2}(r)$, while $\mathcal{S}_{2}$ is the Hall plane of order $r$ (see $[43, \S 13]$ ).

\section{Subfield Class (S0)}

Class (S0) is similar to class (S1): it consists of those affine permutation groups $G$ of rank 3 on $V_{2}\left(q^{3}\right)$ for which $\mathrm{SL}_{2}(q) \unlhd G_{0}$. Let $r:=q^{3}, K:=\mathbb{F}_{r}$ and $\zeta:=\zeta_{r}$ (see $\S 2.3$ ). Let $\left\{v_{1}, v_{2}\right\}$ be a basis of $V_{2}(r)$ on which $\mathrm{GL}_{2}(q)$ acts naturally over $\mathbb{F}_{q}$, and let $U:=\left\langle v_{1}, v_{2}\right\rangle_{\mathbb{F}_{q}}$. By assumption, $G_{0} \leqslant \Gamma L_{2}(r)$, so $G_{0} \leqslant\left(\mathrm{GL}_{2}(q) \circ K^{*}\right):\left\langle\sigma_{r}\right\rangle$ where $\sigma_{r}$ (see $\left.\S 2.3\right)$ acts on $V_{2}(r)$ with respect to $\left\{v_{1}, v_{2}\right\}$. Representatives for the orbits of $G_{0}$ are $v_{1}$ and $v_{1}+\zeta v_{2}$, and $v_{1}^{G_{0}}=\left\{\lambda v: \lambda \in K^{*}, v \in\right.$ $\left.U^{*}\right\}$. Let $\lambda U:=\{\lambda v: v \in U\}$ for $\lambda \in K^{*}$. The orbits of $\mathrm{SL}_{2}(q)$ on $v_{1}^{G_{0}}$ are $\left(\lambda_{1} U\right)^{*}, \ldots,\left(\lambda_{s} U\right)^{*}$, where $\lambda_{1}, \ldots, \lambda_{s}$ is a transversal for $\mathbb{F}_{q}^{*}$ in $K^{*}$ (so $s=q^{2}+q+1$ ).

Here is an equivalent definition for the class (S0). As above, let $r:=q^{3}, K:=\mathbb{F}_{r}$ and $\zeta:=\zeta_{r}$. Let $\left\{v_{1}, v_{2}\right\}$ be a basis of $U:=V_{2}(q)$. The extension of scalars $U \otimes K$ is a 2-dimensional $K$-vector space with basis $\left\{v_{1} \otimes 1, v_{2} \otimes 1\right\}$ on which $\mathrm{GL}_{2}(q) \otimes \mathrm{GL}_{1}(r):\left\langle\sigma_{r}\right\rangle$ acts naturally. Now class (S0) consists of those affine permutation groups $G$ of rank 3 on $U \otimes K$ for which $\mathrm{SL}_{2}(q) \otimes 1 \unlhd G_{0}$. Representatives for the orbits of $G_{0}$ are $v_{1} \otimes 1$ and $v_{1} \otimes 1+v_{2} \otimes \zeta$. Further, we may view $U \otimes K$

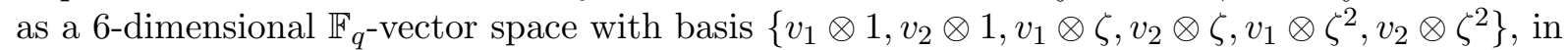
which case $G_{0}$ lies in class (T6). (Note that in class (T6), there exists $t \in \mathrm{GL}_{3}(q)$ such that $t \sigma_{q}$ acts as the Frobenius automorphism of $K$.)

The orbits of $\mathrm{SL}_{2}(q) \otimes 1$ on $\left(v_{1} \otimes 1\right)^{G_{0}}$ are $U^{*} \otimes \lambda_{1}, \ldots, U^{*} \otimes \lambda_{s}$ where $\lambda_{1}, \ldots, \lambda_{s}$ is a transversal for $\mathbb{F}_{q}^{*}$ in $K^{*}$. If we define $\mathcal{L}$ to be the set of translations of $U \otimes \lambda_{i}$ for $1 \leqslant i \leqslant q^{2}+q+1$, then $(U \otimes K, \mathcal{L})$ is one of the proper partial linear spaces described in Example 1.2 and Lemma 10.2(iii), and it is both $K$-independent and $\mathbb{F}_{q}$-independent. However, if we instead define $\mathcal{L}$ to be the set of translations of $v \otimes K=\langle v \otimes 1\rangle_{K}$ for $v \in U^{*}$, then $(U \otimes K, \mathcal{L})$ is not only the other proper partial linear space described in Example 1.2 and Lemma 10.2(iii), but also a partial linear space from Example 1.1 that is $K$-dependent and $\mathbb{F}_{q}$-independent. (In the notation of Example 1.1, for any $G_{0}$ in class (S0), the partial linear space is $\mathcal{S}_{1}$, where $\Delta_{1}$ is the orbit $\left\{\langle v \otimes 1\rangle_{K}: v \in U^{*}\right\}$ of $G_{0}$ on the points of $\mathrm{PG}_{1}\left(q^{3}\right)$.) 
By combining these two viewpoints of class (S0), we obtain the following as an immediate consequence of Lemmas 10.2 and 10.3(i).

Proposition 12.1. Let $G$ be an affine permutation group of rank 3 on $V:=V_{2}\left(q^{3}\right)$ where $\mathrm{SL}_{2}(q) \unlhd G_{0}$ and $q$ is a prime power. Let $K:=\mathbb{F}_{q^{3}}$. Let $\left\{v_{1}, v_{2}\right\}$ be a basis of $V$ on which $\mathrm{GL}_{2}(q)$ acts naturally over $\mathbb{F}_{q}$, and let $U:=\left\langle v_{1}, v_{2}\right\rangle_{\mathbb{F}_{q}}$.

(i) The only $K$-independent $G$-affine proper partial linear space for which 0 and $v_{1}$ are collinear has line set $\mathcal{L}:=\left\{\lambda U+v: \lambda \in K^{*}, v \in V\right\}$.

(ii) The partial linear space $(V, \mathcal{L})$ is described in Example 1.2.

Unfortunately, in contrast to class (S1), we are unable to provide a classification of the $K$ independent $G$-affine proper partial linear spaces for which 0 is collinear with $v_{1}+\zeta v_{2}$ for groups $G$ in class (S0). (Note that the conditions of Theorem 1.4(iv)(c) hold under these assumptions.) Here the group $\mathrm{GL}_{2}(q) \circ K^{*}$ acts regularly on the orbit containing $v_{1}+\zeta v_{2}$, so our usual techniques fail. However, we can classify the $G$-affine proper partial linear spaces when $\mathrm{GL}_{2}(q) \circ K^{*} \leqslant G_{0}$. Since the affine permutation group with stabiliser $\mathrm{GL}_{2}(q) \circ K^{*}$ is transitive of rank 3 , any example that arises for $G_{0}$ also arises for $\mathrm{GL}_{2}(q) \circ K^{*}$, so we assume that $G_{0}=\mathrm{GL}_{2}(q) \circ K^{*}$.

To state our classification, we need some notation. Let $F / E$ be a field extension of degree 2 , and let $\operatorname{End}_{E}(F)$ denote the ring of $E$-endomorphisms of $F$. For $\varphi \in \operatorname{End}_{E}(F)$, we denote the $2 \times 2$ transformation matrix of $\varphi$ with respect to an $E$-basis $\mathcal{B}$ of $F$ by $[\varphi]_{\mathcal{B}}$. In other words, if $\mathcal{B}=\left\{b_{1}, b_{2}\right\}$ and $v=\lambda_{1} b_{1}+\lambda_{2} b_{2}$ for some $\lambda_{1}, \lambda_{2} \in E$, then $\left(\lambda_{1}, \lambda_{2}\right)[\varphi]_{\mathcal{B}}=\left(\mu_{1}, \mu_{2}\right)$ if and only if $v \varphi=\mu_{1} b_{1}+\mu_{2} b_{2}$ where $\mu_{1}, \mu_{2} \in E$. Note that in the following result, it is more convenient not to make the distinction between $K$-independent and $K$-dependent partial linear spaces.

Proposition 12.2. Let $r:=q^{3}, K:=\mathbb{F}_{r}$ and $\zeta:=\zeta_{r}$ where $q$ is a prime power. Let $V:=K^{2}$ and $G:=V:\left(\mathrm{GL}_{2}(q) \circ K^{*}\right)$. Let $x:=(1, \zeta) \in V$. Then $(V, \mathcal{L})$ is a $G$-affine proper partial linear space for which 0 and $x$ are collinear if and only if $\mathcal{L}=L^{G}$ and one of the following holds.

(i) $L=\{\lambda x: \lambda \in F\}$, where $F$ is a subfield of $K$ and $|F|>2$.

(ii) $L=\left\{x \operatorname{diag}\left(\lambda, \lambda^{\tau}\right)^{g}: \lambda \in F\right\}$, where $F$ is a subfield of $\mathbb{F}_{q}, \tau$ is a non-trivial element of $\operatorname{Aut}(F)$, and $g \in \mathrm{GL}_{2}(q)$.

(iii) $L=\left\{x\left[\varphi_{\lambda}\right]_{\mathcal{B}}^{g}: \lambda \in F\right\}$, where $F$ is a field with $\left[F: F \cap \mathbb{F}_{q}\right]=2$, the map $\varphi_{\lambda} \in \operatorname{End}_{F \cap \mathbb{F}_{q}}(F)$ is defined by $v \mapsto \lambda v$ for all $v \in F$, the set $\mathcal{B}$ is an $\left(F \cap \mathbb{F}_{q}\right)$-basis of $F$, and $g \in \mathrm{GL}_{2}(q)$.

Before proving Proposition 12.2, we make some remarks and prove some lemmas.

Remark 12.3. Biliotti et al. [4] consider the case where $G_{0}=\mathrm{GL}_{2}(q) \circ K^{*}$ for $2-(v, k, 1)$ designs in [4, Theorem 32], but they mistakenly assert that their result applies to all rank 3 groups $G$ in class (S0) with $K^{*} \leqslant G_{0}$ by stating that any such group must contain $\mathrm{GL}_{2}(q) \circ K^{*}$ (see [4, p.148]). This is not true, as we now see. Let $K$ be a field of order $r:=q^{3}$ where $q=p^{e}$ and $p$ is prime. Let $S:=\mathrm{SL}_{2}(q) \circ K^{*}$ and $T:=\mathrm{GL}_{2}(q) \circ K^{*}$. Now $T$ is regular on $\left(1, \zeta_{r}\right)^{T}$ and $S$ has index $(2, q-1)$ in $T$, so $S$ has $(2, q-1)$ orbits on $\left(1, \zeta_{r}\right)^{T}$, say $X_{1}, \ldots, X_{(2, q-1)}$. Note also that $(1,0)^{S}=(1,0)^{T}$. Let $g:=\operatorname{diag}\left(\zeta_{q}, 1\right)$ and $\sigma:=\sigma_{r}$. Then $T=S\langle g\rangle$ and $S\langle g, \sigma\rangle / S=\langle S g\rangle \times\langle S \sigma\rangle \simeq C_{(2, q-1)} \times C_{3 e}$. Now $G_{0}$ is the point stabiliser of a rank 3 group in class (S0) that contains $K^{*}$ but not $T$ precisely when the following hold: $q$ is odd; $S \leqslant G_{0} \leqslant S\langle g, \sigma\rangle ; g \notin G_{0}$; and $X_{1}^{h}=X_{2}$ for some $h \in G_{0} \cap\langle g, \sigma\rangle$. Suppose then that $q$ is odd. The subgroups of $S\langle g, \sigma\rangle / S$ not containing $S g$ are cyclic, so it is routine to determine the possibilities for $G_{0}$ : $e$ is even, and either $X_{1}^{\sigma}=X_{1}$, in which case $G_{0}$ is $S\langle g \tau\rangle$ for any $\tau \in\langle\sigma\rangle$ with even order, or $X_{1}^{\sigma}=X_{2}$, in which case $G_{0}$ is $S\langle g \tau\rangle$ for any $\tau \in\left\langle\sigma^{2}\right\rangle$ with even order, or $S\langle\tau\rangle$ for any $\tau \in\langle\sigma\rangle \backslash\left\langle\sigma^{2}\right\rangle$.

Remark 12.4. Let $K$ be a field of order $q^{3}$ where $q=p^{e}$ and $p$ is prime. Let $V:=K^{2}$ and $G:=V:\left(\mathrm{GL}_{2}(q) \circ K^{*}\right)$. Note that the fields $F$ for which $\left[F: F \cap \mathbb{F}_{q}\right]=2$ are precisely the fields of order $p^{2 f}$ where $f$ divides $e$ but $2 f$ does not divide $e$. Let $\mathcal{S}$ be any $G$-affine proper partial linear space that arises from Proposition 12.2(iii) with respect to the field $F$, where $|F|=p^{2 f}$. Now $F$ is not a subfield of $K$. Hence, if $f<e$, then $\mathcal{S}$ cannot be obtained from any $G$-affine $2-\left(q^{6}, p^{2 f}, 1\right)$ design by Propositions 4.2 and 12.1 (in the sense of Remark 1.5 and Lemma 2.11). 
Next we have a lemma that applies to all groups in class (S0).

Lemma 12.5. Let $G$ be an affine permutation group of rank 3 on $V:=V_{2}\left(q^{3}\right)$ where $\mathrm{SL}_{2}(q) \unlhd G_{0}$ and $q$ is a power of a prime $p$. Let $r:=q^{3}$. Let $\left\{v_{1}, v_{2}\right\}$ be a basis of $V$ on which $\mathrm{GL}_{2}(q)$ acts naturally over $\mathbb{F}_{q}$. Let $(V, \mathcal{L})$ be a $G$-affine proper partial linear space for which 0 and $v_{1}+\zeta_{r} v_{2}$ are collinear. If $L \in \mathcal{L}_{0}$, then $L$ is an $\mathbb{F}_{p}$-subspace of $V$ and $|L|$ divides $q^{3}$.

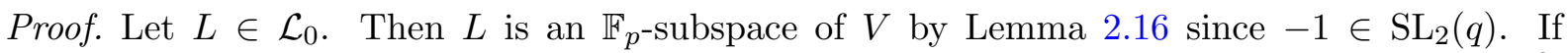
$\lambda v_{1}+\mu v_{2}, \lambda v_{1}+\delta v_{2} \in L^{*}$ for some $\lambda, \mu, \delta \in \mathbb{F}_{r}^{*}$, then $(\mu-\delta) v_{2} \in L$, so $\mu=\delta$. Thus $|L| \leqslant q^{3}$,

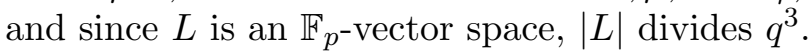

In the following, for a field of size $s$, we denote the ring of $2 \times 2$ matrices over $\mathbb{F}_{s}$ by $\mathrm{M}_{2}(s)$. Note that if $F$ is a field and $\varphi: F \rightarrow \mathrm{M}_{2}(s)$ is an injective ring homomorphism, then $(F \varphi)^{*} \leqslant \mathrm{GL}_{2}(s)$.

Lemma 12.6. Let $r:=q^{3}, K:=\mathbb{F}_{r}$ and $\zeta:=\zeta_{r}$, where $q$ is a prime power. Let $V:=K^{2}$ and $G:=V:\left(\mathrm{GL}_{2}(q) \circ K^{*}\right)$. Let $x:=(1, \zeta) \in V$. The following are equivalent.

(i) $(V, \mathcal{L})$ is a $G$-affine proper partial linear space for which 0 and $x$ are collinear.

(ii) $\mathcal{L}=L^{G}$ where $L=\{x(\lambda \varphi): \lambda \in F\}$ for some field $F$ with $|F|>2$ and injective ring homomorphism $\varphi: F \rightarrow \mathrm{M}_{2}(r)$ such that $(F \varphi)^{*} \leqslant \mathrm{GL}_{2}(q)$ or $K^{*}$.

Proof. Let $(V, \mathcal{L})$ be a $G$-affine proper partial linear space for which 0 and $x$ are collinear. Let $L$ be the line containing 0 and $x$. Since $G$ is transitive of rank 3 on $V$, the line set $\mathcal{L}=L^{G}$. By Lemma 12.5, $L$ is an $\mathbb{F}_{p}$-subspace of $V$ and $|L|=p^{m}$ for some $m \leqslant 3 e$ where $q=p^{e}$ and $p$ is prime. Let $B:=L^{*}$. By Lemma $2.6, B$ is a block of $G_{0}$ on $x^{G_{0}}$. Let $H:=G_{0, B}$. Note that $|H|=p^{m}-1$ since $G_{0}$ is regular on $x^{G_{0}}$. In order to establish (ii), since $B=\left\{x^{h}: h \in H\right\}$, it suffices to prove that $H \cup\{0\}$ is a field (as a subring of $\mathrm{M}_{2}(r)$ ) and that $H \leqslant \mathrm{GL}_{2}(q)$ or $K^{*}$.

Observe that $H \cap K^{*}=\left\{\lambda \in K^{*}: \lambda x \in B\right\}$, for if $\lambda \in K^{*}$ and $\lambda x \in B$, then $x^{\lambda} \in B^{\lambda} \cap B$, and since $\lambda \in G_{0}$, it follows that $B^{\lambda}=B$, so $\lambda \in H$. Since $L$ is closed under addition, it follows that $\left(H \cap K^{*}\right) \cup\{0\}$ is closed under addition. Clearly $H \cap K^{*}$ is a subgroup of the abelian group $K^{*}$, so $\left(H \cap K^{*}\right) \cup\{0\}$ is a field and $\left|H \cap K^{*}\right|=p^{f}-1$ for some $f$ that divides $3 e$. Since $\left|H \cap K^{*}\right|$ divides $|H|$, it follows that $f$ divides $m$. If $f=m$, then $H \leqslant K^{*}$, in which case $H \cup\{0\}$ is a field and (ii) holds, so we may assume that $f<m$.

The group $H /\left(H \cap K^{*}\right)$ is isomorphic to a subgroup of $\mathrm{GL}_{2}(q) /\left(\mathrm{GL}_{2}(q) \cap K^{*}\right)$, which has order $q\left(q^{2}-1\right)$, so $p^{m}-1$ divides $\left(p^{f}-1\right)\left(p^{2 e}-1\right)$. We claim that $m$ divides $2 e$. We may assume that $m>2$. If the pair $(p, m) \neq(2,6)$, then $p^{m}-1$ has a primitive prime divisor, say $t$ (see [58] or [37, Theorem 5.2.14]). Now $t$ divides $\left(p^{f}-1\right)\left(p^{2 e}-1\right)$, but $f<m$, so $t$ divides $p^{2 e}-1$. Since $p$ has order $m$ in $(\mathbb{Z} / t \mathbb{Z})^{*}$, we conclude that $m$ divides $2 e$, as desired. Otherwise, $(p, m)=(2,6)$. Now $f \in\{1,2,3\}$, and 63 divides $\left(2^{f}-1\right)\left(4^{e}-1\right)$. If $f=1$ or 2 , then 7 divides $4^{e}-1$, and 4 has order 3 in $(\mathbb{Z} / 7 \mathbb{Z})^{*}$, so 3 divides $e$. Thus $m$ divides $2 e$. If instead $f=3$, then 9 divides $4^{e}-1$, and 4 has order 3 in $(\mathbb{Z} / 9 \mathbb{Z})^{*}$, so $m$ divides $2 e$. Hence we have established the claim.

Next we claim that $H \leqslant \mathrm{GL}_{2}(q)$. Since $m$ divides $2 e$, the order of $H$ divides $q^{2}-1$. Let $h \in H$. Now $h=\lambda g$ for some $g \in \mathrm{GL}_{2}(q)$ and $\lambda \in K^{*}$. Since $h$ has order dividing $q^{2}-1$, it follows that $g^{q^{2}-1} \lambda^{q^{2}-1}=1$. Since $g \in \mathrm{GL}_{2}(q)$, either $g$ has one Jordan block and the order of $g$ divides $p(q-1)$, or $g$ has two Jordan blocks (over an extension field of $\mathbb{F}_{q}$ with degree at most 2) and the order of $g$ divides $q^{2}-1$. Thus $\lambda^{p\left(q^{2}-1\right)}=1$, so the order of $\lambda$ divides $\operatorname{gcd}\left(p\left(q^{2}-1\right), q^{3}-1\right)=q-1$. Hence $\lambda \in \mathrm{GL}_{2}(q)$, and the claim follows.

It remains to show that $H \cup\{0\}$ is a field. To do so, it suffices to prove that $\operatorname{det}(1+h) \neq 0$ for all $h \in H \backslash\{-1\}$. Indeed, suppose that this statement holds, and let $h_{1}, h_{2} \in H$. We claim that $h_{1}+h_{2} \in H \cup\{0\}$, in which case it will follow that $H \cup\{0\}$ is a field since $H$ is a subgroup of $\mathrm{GL}_{2}(q)$ and any finite division ring is a field by Wedderburn's theorem. If $h_{1}+h_{2}=0$, then we are done, so assume otherwise. Now $h_{1}^{-1} h_{2} \neq-1$, so $\operatorname{det}\left(1+h_{1}^{-1} h_{2}\right) \neq 0$, whence $\operatorname{det}\left(h_{1}+h_{2}\right) \neq 0$. Since $H \leqslant \mathrm{GL}_{2}(q)$, it follows that $h_{1}+h_{2} \in \mathrm{GL}_{2}(q)$. Now $x^{h_{1}+h_{2}} \neq 0$, and $L$ is closed under addition, so $x^{h_{1}+h_{2}}=x^{h_{1}}+x^{h_{2}} \in B$. Thus there exists $k \in H$ such that $x^{h_{1}+h_{2}}=x^{k}$, but $\mathrm{GL}_{2}(q)$ is semiregular on $x^{G_{0}}$, so $h_{1}+h_{2}=k \in H$, as desired. 
Let $h \in H \backslash\{-1\}$, and suppose for a contradiction that $\operatorname{det}(1+h)=0$. Let $\lambda$ and $\mu$ be the eigenvalues of $h$ in some field extension of $\mathbb{F}_{q}$. Since $|H|=p^{m}-1$, the order of $h$ is not divisible by $p$, so $h$ has two Jordan blocks. Thus $h^{g}=\operatorname{diag}(\lambda, \mu)$ for some $g \in \mathrm{GL}_{2}\left(\mathbb{F}_{q}(\lambda)\right)$. Now $(1+h)^{g}=1+h^{g}$, so $\operatorname{det}\left(1+h^{g}\right)=0$. Thus $\lambda=-1$ or $\mu=-1$. Without loss of generality, we may assume that $\lambda=-1$. In particular, $\mu \in \mathbb{F}_{q}^{*}$, so $g \in \mathrm{GL}_{2}(q)$. Let $y:=x^{g}$, and write $y=\left(\lambda_{1}, \lambda_{2}\right)$ where $\lambda_{1}, \lambda_{2} \in K^{*}$. Now $B^{g}$ is a block of $G_{0}$ containing $y$, and $h^{g} \in G_{0, B^{g}}$,

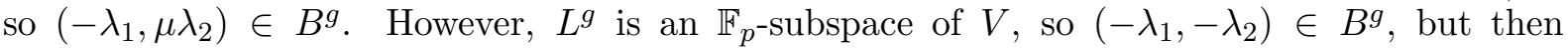
$\left(0, \lambda_{2}(\mu+1)\right) \in L^{g}$. Since $B^{g} \subseteq x^{G_{0}}$, it follows that $\mu=-1$, but then $h=-1$, a contradiction. Thus we have established (ii).

Conversely, suppose that $\mathcal{L}=L^{G}$ where $L=\{x(\lambda \varphi): \lambda \in F\}$ for some field $F$ with $|F|>2$ and injective ring homomorphism $\varphi: F \rightarrow \mathrm{M}_{2}(r)$ such that $(F \varphi)^{*} \leqslant \mathrm{GL}_{2}(q)$ or $K^{*}$. Let $H:=(F \varphi)^{*}$ and $B:=L^{*}=x^{H}$. Since $G_{0}$ acts regularly on $x^{G_{0}}$, it follows that $B$ is a block of

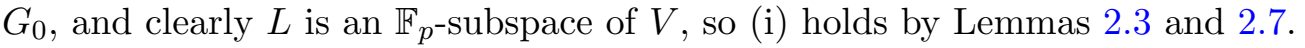

Now we consider the possibilities for the field $F$ and the injective ring homomorphism $\varphi$ in the case where $\varphi: F \rightarrow \mathrm{M}_{2}(q)$. There are two natural ways of defining such a ring homomorphism. First, let $F$ be a subfield of $\mathbb{F}_{q}$, let $\tau \in \operatorname{Aut}(F)$ and define $\varphi: F \rightarrow \mathrm{M}_{2}(q)$ by $\lambda \mapsto \operatorname{diag}\left(\lambda, \lambda^{\tau}\right)$. Clearly $\varphi$ is an injective ring homomorphism. Second, let $E$ be a subfield of $\mathbb{F}_{q}$, and let $F$ be a field extension of $E$ of degree 2. Define $\varphi: F \rightarrow \operatorname{End}_{E}(F)$ by $\lambda \mapsto \varphi_{\lambda}$ where $\varphi_{\lambda}: v \mapsto \lambda v$ for all $v \in F$. This is an injective ring homomorphism, and by fixing a basis for the $E$-vector space $F$, we see that $\operatorname{End}_{E}(F) \simeq \mathrm{M}_{2}(E)$, and $\mathrm{M}_{2}(E)$ is a subring of $\mathrm{M}_{2}(q)$, so $\varphi$ naturally gives us an injective ring homomorphism from $F$ to $\mathrm{M}_{2}(q)$. It is well known that, up to conjugacy in $\mathrm{GL}_{2}(q)$, there are no other ways of embedding $F$ in $\mathrm{M}_{2}(q)$. We state this result explicitly below, and since we could not find a reference for it in this form, we prove it for completeness.

Lemma 12.7. Let $F$ be a field, and let $\varphi: F \rightarrow \mathrm{M}_{2}(q)$ be an injective ring homomorphism where $q$ is a prime power. Let $H:=(F \varphi)^{*} \leqslant \mathrm{GL}_{2}(q)$, and let $H=\langle h\rangle$. Let $\varepsilon$ be an eigenvalue of $h$ in some field extension of $\mathbb{F}_{q}$. Then $F^{*} \simeq\langle\varepsilon\rangle$, and we may view $F$ as a subfield of $\mathbb{F}_{q}(\varepsilon)$. Further, exactly one of the following holds, where $E:=F \cap \mathbb{F}_{q}$.

(i) $\varepsilon \in \mathbb{F}_{q}$, and $h$ is conjugate in $\mathrm{GL}_{2}(q)$ to $\operatorname{diag}\left(\varepsilon, \varepsilon^{\tau}\right)$ for some $\tau \in \operatorname{Aut}(F)$.

(ii) $[F: E]=2$, and $h$ is conjugate in $\mathrm{GL}_{2}(q)$ to $\left[\varphi_{\varepsilon}\right]_{\mathcal{B}}$, where $\varphi_{\varepsilon} \in \operatorname{End}_{E}(F)$ is defined by $v \mapsto \varepsilon v$ for all $v \in F$, and $\mathcal{B}:=\{1, \varepsilon\}$ is an $E$-basis of $F$.

Proof. Let $p$ be the characteristic of $\mathbb{F}_{q}$. Since $\varphi$ maps the identity of $F$ to the identity of $\mathrm{M}_{2}(q)$, it follows that $F$ also has characteristic $p$. In particular, the order of $h$ is not divisible by $p$. Let $f(X) \in \mathbb{F}_{q}[X]$ be the characteristic polynomial of $h$. Then $f(X)=(X-\varepsilon)(X-\delta)$ for some $\delta \in \mathbb{F}_{q}(\varepsilon)$. Since the order of $h$ is not divisible by $p$, it must have two Jordan blocks, so $h$ is conjugate in $\mathrm{GL}_{2}\left(\mathbb{F}_{q}(\varepsilon)\right)$ to $g:=\operatorname{diag}(\varepsilon, \delta)$.

Let $\ell$ and $m$ be the order of $\varepsilon$ and $\delta$ in $\mathbb{F}_{q}(\varepsilon)^{*}$, respectively. We may assume that $\ell \leqslant m$. Now $\langle g\rangle \cup\{0\}$ is conjugate to $F \varphi$, so it is closed under addition. In particular, it contains the matrix $1-\operatorname{diag}\left(1, \delta^{\ell}\right)=\operatorname{diag}\left(0,1-\delta^{\ell}\right)$, so $1=\delta^{\ell}$, and it follows that $m=\ell=|F|-1$. Thus $F^{*} \simeq\langle\varepsilon\rangle \simeq\langle\delta\rangle$, and we may view $F$ as the subfield of $\mathbb{F}_{q}(\varepsilon)$ that contains $\varepsilon$ and $\delta$. Now $\delta=\varepsilon^{n}$ for some integer $n$, so $\langle g\rangle=\left\{\operatorname{diag}\left(\lambda, \lambda^{n}\right): \lambda \in F^{*}\right\}$, and it follows that $(\lambda+\mu)^{n}=\lambda^{n}+\mu^{n}$ for all $\lambda, \mu \in F$. Thus there exists $\tau \in \operatorname{Aut}(F)$ such that $\delta=\varepsilon^{\tau}$, and (i) holds when $\varepsilon \in \mathbb{F}_{q}^{*}$.

Suppose instead that $\varepsilon \notin \mathbb{F}_{q}$. As in the statement of the lemma, let $E:=F \cap \mathbb{F}_{q}$. Now $f(X) \in E[X]$ is irreducible, so $f(X)$ is the minimal polynomial of $\varepsilon$ over $E$, but $F=E(\varepsilon)$, so $[F: E]=2$. Thus $\mathcal{B}$ is an $E$-basis for $F$, and there exist $a, b \in E$ such that $\varphi_{\varepsilon}$ maps $\varepsilon$ to $a \cdot 1+b \cdot \varepsilon$. Now $\varepsilon$ is a root of $X^{2}-b X-a \in E[X]$, so $f(X)=X^{2}-b X-a$. Since $X^{2}-b X-a$ is also the characteristic polynomial of $\left[\varphi_{\varepsilon}\right]_{\mathcal{B}}$, we conclude that $\left[\varphi_{\varepsilon}\right]_{\mathcal{B}}$ is conjugate in $\operatorname{GL}_{2}\left(\mathbb{F}_{q}(\varepsilon)\right.$ ) to $\operatorname{diag}(\varepsilon, \delta)$. Hence $h$ and $\left[\varphi_{\varepsilon}\right]_{\mathcal{B}}$ are conjugate in $\mathrm{GL}_{2}\left(\mathbb{F}_{q}(\varepsilon)\right)$, but both matrices have entries in $\mathbb{F}_{q}$, so they are also conjugate in $\mathrm{GL}_{2}(q)$. 
Proof of Proposition 12.2. Let $(V, \mathcal{L})$ be a $G$-affine proper partial linear space for which 0 and $x$ are collinear. By Lemma 12.6, $\mathcal{L}=L^{G}$ where $L=\{x(\lambda \varphi): \lambda \in F\}$ for some field $F$ with $|F|>2$ and injective ring homomorphism $\varphi: F \rightarrow \mathrm{M}_{2}(r)$ such that $(F \varphi)^{*} \leqslant \mathrm{GL}_{2}(q)$ or $K^{*}$. If $(F \varphi)^{*} \leqslant K^{*}$, then (i) holds, so we may assume that $(F \varphi)^{*} \leqslant \mathrm{GL}_{2}(q)$. Let $(F \varphi)^{*}=\langle h\rangle$, and let $\varepsilon$ be an eigenvalue of $h$ in some field extension of $\mathbb{F}_{q}$. By Lemma 12.7, $F^{*} \simeq\langle\varepsilon\rangle$, we may view $F$ as a subfield of $\mathbb{F}_{q}(\varepsilon)$, and Lemma 12.7(i) or (ii) holds. First suppose that Lemma 12.7(i) holds. Then $\varepsilon \in \mathbb{F}_{q}$ and $h=\operatorname{diag}\left(\varepsilon, \varepsilon^{\tau}\right)^{g}$ for some $\tau \in \operatorname{Aut}(F)$ and $g \in \mathrm{GL}_{2}(q)$. If $\tau=1$, then (i) holds. Otherwise, (ii) holds. Thus we may assume that Lemma 12.7(ii) holds. Let $E:=F \cap \mathbb{F}_{q}$. Then $[F: E]=2$, and $h$ is conjugate in $\mathrm{GL}_{2}(q)$ to $\left[\varphi_{\varepsilon}\right]_{\mathcal{B}}$, where $\varphi_{\varepsilon} \in \operatorname{End}_{E}(F)$ is defined by $v \mapsto \varepsilon v$ for all $v \in F$, and $\mathcal{B}=\{1, \varepsilon\}$ is an $E$-basis of $F$. Thus (iii) holds.

Conversely, suppose that $\mathcal{L}=L^{G}$ where $L$ is given by (i), (ii) or (iii). In each case, we claim that that $L=\{x(\lambda \varphi): \lambda \in F\}$ for some injective ring homomorphism $\varphi: F \rightarrow \mathrm{M}_{2}(r)$ such that $(F \varphi)^{*} \leqslant \mathrm{GL}_{2}(q)$ or $K^{*}$. If the claim holds, then since $|F|>2$ in (i)-(iii), Lemma 12.6 implies that $(V, \mathcal{L})$ is a $G$-affine proper partial linear space for which 0 and $x$ are collinear, as desired. If (i) holds, then we define $\varphi$ by $\lambda \mapsto \operatorname{diag}(\lambda, \lambda)$ for all $\lambda \in F$, and the claim holds. If (ii) holds, then we define $\varphi$ by $\lambda \mapsto \operatorname{diag}\left(\lambda, \lambda^{\tau}\right)^{g}$ for all $\lambda \in F$, and the claim holds. If (iii) holds, then we define $\varphi$ by $\lambda \mapsto\left[\varphi_{\lambda}\right]_{\mathcal{B}}^{g}$ for all $\lambda \in F$, and the claim holds.

Proposition 12.2 provides a classification of the $G$-affine proper partial linear spaces for which 0 and $(1, \zeta)$ are collinear when $G_{0}$ lies in class $(\mathrm{S} 0)$ and $\mathrm{GL}_{2}(q) \circ \mathbb{F}_{q^{3}}^{*} \leqslant G_{0}$. However, we suspect that the situation is much more complicated in general. To illustrate this, we now provide a complete classification of the partial linear spaces that arise when $q=4$.

Example 12.8. Let $q:=4, r:=q^{3}, K:=\mathbb{F}_{r}, \sigma:=\sigma_{r}, \zeta:=\zeta_{r}, x:=(1, \zeta)$ and $V:=K^{2}$. Let $S:=\mathrm{GL}_{2}(q)$. By a computation in MAGMA, the point stabiliser of an affine permutation group of rank 3 on $V$ in (S0) is either $S\left\langle\zeta, \sigma^{i}\right\rangle$ for $i \in\{1,2,3,6\}$, or conjugate to $S\left\langle\zeta^{3}, \zeta^{7} \sigma^{2}\right\rangle$. Note that $S\left\langle\zeta^{3}, \zeta^{7} \sigma^{2}\right\rangle$ does not contain $S \circ K^{*}=S\langle\zeta\rangle$. Recall that we may view $S\langle\zeta, \sigma\rangle$ as a member of class (T6) and therefore as a subgroup of $W_{0}:=\left(S \otimes \mathrm{GL}_{3}(q)\right)$ : Aut $\left(\mathbb{F}_{q}\right)$.

We claim that Table 7 contains a complete list of representatives for the isomorphism classes of $X$-affine proper partial linear spaces for which 0 and $x$ are collinear, where $X$ is an affine permutation group of rank 3 on $V$ in (S0). For each such partial linear space $\mathcal{S}$, we prove that $\operatorname{Aut}(\mathcal{S})=V: \operatorname{Aut}(\mathcal{S})_{0}$, and we list the following in Table 7: the line-size $k ; \operatorname{Aut}(\mathcal{S})_{0}$; and when $S\langle\zeta\rangle \leqslant \operatorname{Aut}(\mathcal{S})_{0}$, a reference to Proposition 12.2.

\begin{tabular}{ccccc}
\hline$k$ & $\mathcal{S}$ & $\operatorname{Aut}(\mathcal{S})_{0}$ & 12.2 & Notes \\
\hline 4 & $\mathcal{S}_{4,1}$ & $W_{0}$ & (i) & $K$-dependent \\
& $\mathcal{S}_{4,2}$ & $S\langle\zeta, \sigma\rangle$ & (ii) & \\
& $\mathcal{S}_{4,3}$ & $S\left\langle\zeta, \sigma^{3}\right\rangle$ & (ii) & \\
& $\mathcal{S}_{4,4}$ & $S\langle\zeta\rangle$ & (ii) & \\
& $\mathcal{S}_{4, \ell}$ & $S\left\langle\zeta^{3}, \zeta^{7} \sigma^{2}\right\rangle$ & n/a & $5 \leqslant \ell \leqslant 7$ \\
\hline 8 & $\mathcal{S}_{8,1}$ & $S\langle\zeta, \sigma\rangle$ & (i) & $K$-dependent \\
\hline 16 & $\mathcal{S}_{16,1}$ & $S\langle\zeta\rangle$ & (iii) & \\
& $\mathcal{S}_{16, \ell}$ & $S\left\langle\zeta^{3}, \zeta^{7} \sigma^{2}\right\rangle$ & n/a & $2 \leqslant \ell \leqslant 3$ \\
\hline 64 & $\mathcal{S}_{64,1}$ & $S\langle\zeta, \sigma\rangle$ & (i) & $K$-dependent \\
\hline
\end{tabular}

TABLE 7. Partial linear spaces $\mathcal{S}$ when $q=4$ and 0 is collinear with $\left(1, \zeta_{64}\right)$

Observe in Table 7 that there are five pairwise non-isomorphic partial linear spaces $\mathcal{S}$ for which $\operatorname{Aut}(\mathcal{S})_{0}=S\left\langle\zeta^{3}, \zeta^{7} \sigma^{2}\right\rangle$, none of which are isomorphic to a partial linear space described by Proposition 12.2. Further, there are three pairwise non-isomorphic partial linear spaces that are described by Proposition 12.2(ii), necessarily with $F=\mathbb{F}_{4}$ and $\tau=\sigma_{4}$.

Now we prove the claim. Let $G:=V: S\langle\zeta\rangle, H:=V: S\left\langle\zeta^{3}, \zeta^{7} \sigma^{2}\right\rangle, N:=V: S\langle\zeta, \sigma\rangle$ and $W:=$ $V: W_{0}$. Then $N_{N_{0}}\left(H_{0}\right)=H_{0}\left\langle\sigma^{2}\right\rangle=G_{0}\left\langle\sigma^{2}\right\rangle$ and $\left|G_{0}\right|=\left|H_{0}\right|$. Let $\mathcal{G}:=\left\{H, G, G\left\langle\sigma^{2}\right\rangle, G\left\langle\sigma^{3}\right\rangle, N\right\}$. 
For $X \in\{G, H\}$, let $\mathcal{B}_{X}$ denote the set of non-trivial blocks $B$ of $X_{0}$ on $x^{N_{0}}$ such that $x \in B$ and $B \cup\{0\}$ is an $\mathbb{F}_{2}$-subspace of $V$. Let $Y \in \mathcal{G}$, and recall from Lemmas 2.6 and 12.5 that if $(V, \mathcal{L})$ is a $Y$-affine proper partial linear space and $L \in \mathcal{L}_{0} \cap \mathcal{L}_{x}$, then $L^{*} \in \mathcal{B}_{X}$ for some $X \in\{G, H\}$. Conversely, if $X \in\{G, H\}$ and $B \in \mathcal{B}_{X}$, then $\left(V,(B \cup\{0\})^{X}\right)$ is an $X$-affine proper partial linear space by Lemmas 2.3 and 2.7. Therefore, in order to find all of the relevant partial linear spaces, it suffices to determine the sets $\mathcal{B}_{X}$. By a computation in Magma, $\mathcal{B}_{G}=\mathcal{B}_{H}$, and $\mathcal{B}_{G}$ consists of 19 blocks. Three of these correspond to $K$-dependent $N$-affine partial linear spaces with line-sizes 4,8 and 64 , which we denote by $\mathcal{S}_{4,1}, \mathcal{S}_{8,1}$ and $\mathcal{S}_{64,1}$, respectively. Note that these are described by Proposition 12.2(i) with $F=\mathbb{F}_{k}$ where $k$ is the line-size. By a computation in MaGma, $\operatorname{Aut}\left(\mathcal{S}_{8,1}\right)=\operatorname{Aut}\left(\mathcal{S}_{64,1}\right)=N$. We will determine $\operatorname{Aut}\left(\mathcal{S}_{4,1}\right)$ below.

Of the remaining sixteen blocks in $\mathcal{B}_{G}$, six have size 15 and ten have size 3 . First we consider the blocks with size 15. By a computation in MAGMA, the group $\langle\sigma\rangle$ permutes the six $G$-affine partial linear spaces with line-size 16 transitively, so all of these examples are isomorphic; let $\mathcal{S}_{16,1}$ denote one of these. Further, the group $\langle\sigma\rangle$ does not act on the six $H$-affine partial linear spaces with line-size 16 , while $\left\langle\sigma^{2}\right\rangle$ has two orbits of size 3 ; let $\mathcal{S}_{16,2}$ and $\mathcal{S}_{16,3}$ denote orbit representatives of these. By a computation in Magma, $\operatorname{Aut}\left(\mathcal{S}_{16,1}\right)=G$, while $\operatorname{Aut}\left(\mathcal{S}_{16,2}\right)=\operatorname{Aut}\left(\mathcal{S}_{16,3}\right)=H$, and the partial linear spaces $\mathcal{S}_{16,1}, \mathcal{S}_{16,2}$ and $\mathcal{S}_{16,3}$ are pairwise non-isomorphic. Note that $\mathcal{S}_{16,1}$ must have the form of Proposition 12.2(iii) with $F=\mathbb{F}_{16}$.

It remains to consider the blocks with size 3 . Recall that $\mathcal{S}_{4,1}$ denotes the $K$-dependent $N$ affine partial linear space with line-size 4 . By a computation in MAGMA, the group $\langle\sigma\rangle$ has orbit sizes 1,3 and 6 on the ten $G$-affine $K$-independent partial linear spaces with line-size 4 . In particular, $N$ is an automorphism group of the partial linear space in the orbit of size 1; let $\mathcal{S}_{4,2}$ denote this partial linear space, and let $\mathcal{S}_{4,3}$ and $\mathcal{S}_{4,4}$ be representatives of the orbits with size 3 and 6 , respectively. Note that $\mathcal{S}_{4, j}$ must have the form of Proposition 12.2(ii) with $F=\mathbb{F}_{4}$ and $\tau=\sigma_{4}$ for $2 \leqslant j \leqslant 4$. By a computation in MAGMA, $\langle\sigma\rangle$ does not act on the nine $H$-affine $K$-independent partial linear spaces with line-size 4 that are not equal to $\mathcal{S}_{4,2}$, while $\left\langle\sigma^{2}\right\rangle$ has orbit sizes 3,3 and 3 ; let $\mathcal{S}_{4,5}, \mathcal{S}_{4,6}$ and $\mathcal{S}_{4,7}$ be orbit representatives of these.

Let $j \in\{1, \ldots, 7\}$. We wish to determine $\operatorname{Aut}\left(\mathcal{S}_{4, j}\right)$, but we are unable to do so directly using Magma. By Lemma 10.9, Aut $\left(\mathcal{S}_{4, j}\right) \leqslant W$. In particular, $\operatorname{Aut}\left(\mathcal{S}_{4,1}\right)=W$, so we assume that $j \neq 1$. Recall that $S \unlhd W_{0}$ and $S\left\langle\zeta^{3}\right\rangle \leqslant \operatorname{Aut}\left(\mathcal{S}_{4, j}\right)_{0}$. By a computation in Magma, there are exactly four subgroups of $W_{0}$ (up to conjugacy) that contain $S$ and have two orbits on $V^{*}$ but are not conjugate to a subgroup of $N_{0}$. Further, if $B$ is a block of one of these four groups on $x^{N_{0}}$ for which $x \in B$ and $B \cup\{0\}$ is an $\mathbb{F}_{2}$-subspace of $V$ with size 4, then $B \cup\{0\}=\langle x\rangle_{\mathbb{F}_{4}}$. However, $j \neq 1$, so $\operatorname{Aut}\left(\mathcal{S}_{4, j}\right)_{0}$ is conjugate in $W_{0}$ to a subgroup of $N_{0}$. By a computation in Magma, $N_{0}$ is the only conjugate of $N_{0}$ in $W_{0}$ that contains $\zeta^{3}$. Thus $\operatorname{Aut}\left(\mathcal{S}_{4, j}\right)_{0} \leqslant N_{0}$. Now we can deduce from the actions of $\langle\sigma\rangle$ given above that $\operatorname{Aut}\left(\mathcal{S}_{4, j}\right)$ is as described in Table 7. By a computation in Magma, $G\left\langle\sigma^{2}\right\rangle$ is the normaliser of $H$ in $S_{4096}$, and $G$ and $H$ are not permutation isomorphic. Thus the partial linear spaces $\mathcal{S}_{4, j}$ are pairwise non-isomorphic for $1 \leqslant j \leqslant 7$.

\section{The IMPRIMitive ClASS (I)}

Let $V:=V_{n}(p) \oplus V_{n}(p)$ where $n \geqslant 1$ and $p$ is prime. We adopt the following notation throughout this section. The stabiliser of the decomposition of $V$ in $\mathrm{GL}_{2 n}(p)$ is $\mathrm{GL}_{n}(p) \imath\langle\tau\rangle$, where $\tau$ is the involution in $\mathrm{GL}_{2 n}(p)$ defined by $\left(u_{1}, u_{2}\right)^{\tau}=\left(u_{2}, u_{1}\right)$ for all $u_{1}, u_{2} \in V_{n}(p)$. We write $V_{1}:=\left\{(u, 0): u \in V_{n}(p)\right\}$ and $V_{2}:=\left\{(0, u): u \in V_{n}(p)\right\}$, and for $G_{0} \leqslant \mathrm{GL}_{n}(p) \imath\langle\tau\rangle$ and $i \in\{1,2\}$, we write $G_{0}^{i}$ for the image of the projection of $G_{0, V_{1}}=G_{0} \cap\left(\mathrm{GL}_{n}(p) \times \mathrm{GL}_{n}(p)\right)$ onto the $i$-th factor of $\mathrm{GL}_{n}(p) \times \mathrm{GL}_{n}(p)$.

If $G$ is an affine permutation group of rank 3 on $V$, then the orbits of $G_{0}$ on $V^{*}$ are $V_{1}^{*} \cup V_{2}^{*}$ and $V_{n}(p)^{*} \times V_{n}(p)^{*}$. In particular, $G$ is primitive if and only if $p^{n}>2$ by Lemma 2.2. Further, $G_{0}^{i}$ is transitive on $V_{n}(p)^{*}$ for both $i$, and $G_{0, V_{1}}$ is an index 2 subgroup of $G_{0}$. Now $G_{0}=G_{0, V_{1}}\langle(t, s) \tau\rangle$ for some $t, s \in \mathrm{GL}_{n}(p)$. Conjugating $G_{0}$ by $\left(s^{-1}, 1\right)$ if necessary, we may assume that $s=1$, in which case $G_{0}^{1}=G_{0}^{2}$ and $G_{0} \leqslant G_{0}^{1} \imath\langle\tau\rangle$ since $((t, 1) \tau)^{2}=(t, t)$. 
If $H_{0} \leqslant \Gamma \mathrm{L}_{m}(q)$ where $q^{m}=p^{n}$ and $H_{0}$ is transitive on $V_{n}(p)^{*}$, then $V:\left(H_{0}\langle\langle\tau\rangle)\right.$ is a rank 3 subgroup of $V:\left(\mathrm{GL}_{n}(p)\langle\langle\tau\rangle)\right.$. Note that $\Gamma \mathrm{L}_{m}(q)\left\langle\langle\tau\rangle\right.$ is not a subgroup of $\Gamma \mathrm{L}_{2 m}(q)$ when $q \neq p$. Indeed, $\left(\Gamma \mathrm{L}_{m}(q) \imath\langle\tau\rangle\right) \cap \Gamma \mathrm{L}_{2 m}(q)=\left\{\left(g_{1} \sigma, g_{2} \sigma\right): g_{1}, g_{2} \in \mathrm{GL}_{m}(q), \sigma \in \operatorname{Aut}\left(\mathbb{F}_{q}\right)\right\}\langle\tau\rangle$, which is isomorphic to $\left(\mathrm{GL}_{m}(q)<\langle\tau\rangle\right)$ : $\operatorname{Aut}\left(\mathbb{F}_{q}\right)$.

In previous sections, the rank 3 groups under consideration were all subgroups of $\mathrm{A}_{m}(q)$ for some field extension $\mathbb{F}_{q}$ of $\mathbb{F}_{p}$, in which case it was logical to consider $\mathbb{F}_{q}$-dependent and $\mathbb{F}_{q}$-independent partial linear spaces separately. However, since this does not naturally occur for all groups in a particular subclass of (I), we will not make this distinction here. Nevertheless, we will see that there are two infinite families of $\mathbb{F}_{q}$-dependent proper partial linear spaces for each field $\mathbb{F}_{q}$ such that $q^{m}=p^{n}$. In fact, one of these examples admits $V:\left(\Gamma L_{m}(q) \imath\langle\tau\rangle\right)$ as an automorphism group, even though $\Gamma \mathrm{L}_{m}(q) \imath\langle\tau\rangle$ is not $\mathbb{F}_{q}$-semilinear when $q \neq p$.

In this section, we prove the following two propositions. For the first, recall the definition of the cartesian product of two partial linear spaces that was given in $\S 2.7$, and observe that the partial linear space in (i) below is that of Example 1.3.

Proposition 13.1. Let $G$ be an affine permutation group of rank 3 on $V:=V_{n}(p) \oplus V_{n}(p)$ where $n \geqslant 1, p$ is prime and $G_{0} \leqslant \mathrm{GL}_{n}(p) \imath\langle\tau\rangle$. Then $\mathcal{S}$ is a $G$-affine proper partial linear space in which $\mathcal{S}(0)=V_{1}^{*} \cup V_{2}^{*}$ if and only if one of the following holds.

(i) $\mathcal{S}$ is the $p^{n} \times p^{n}$ grid where $p^{n}>2$.

(ii) $\mathcal{S}=\mathrm{AG}_{m}(q) \square \mathrm{AG}_{m}(q)$ and $G_{0} \leqslant \Gamma \mathrm{L}_{m}(q) \imath\langle\tau\rangle$, where $q^{m}=p^{n}, m \geqslant 2$ and $q>2$.

(iii) $\mathcal{S} \simeq \mathcal{S}^{\prime} \square \mathcal{S}^{\prime}$ and one of the following holds.

(a) $\mathcal{S}^{\prime}$ is the nearfield plane of order 9 and $G_{0} \leqslant N_{\mathrm{GL}_{4}(3)}\left(D_{8} \circ Q_{8}\right) 2\langle\tau\rangle$. Here $p^{n}=3^{4}$.

(b) $\mathcal{S}^{\prime}$ is the Hering plane of order 27 or either of the two Hering spaces with line-size 9 , and $G_{0}=\mathrm{SL}_{2}(13) \imath\langle\tau\rangle$. Here $p^{n}=3^{6}$.

Remark 13.2. The partial linear space $\mathcal{S}:=\mathrm{AG}_{m}(q) \square \mathrm{AG}_{m}(q)$ in Proposition 13.1(ii) has line set $\left\{\langle u\rangle_{\mathbb{F}_{q}}+v: u \in V_{1}^{*} \cup V_{2}^{*}, v \in V\right\}$, so $\mathcal{S}$ is an $\mathbb{F}_{q^{-}}$-dependent partial linear space. In particular, $\mathcal{S}$ is described in Example 1.1 with respect to the triple $\left(\operatorname{AGL}_{m}(q) \imath\langle\tau\rangle, 2 m, q\right)$, which satisfies Hypothesis 3.6(iii). However, $\operatorname{A\Gamma L}_{m}(q) \imath\langle\tau\rangle \leqslant \operatorname{Aut}(\mathcal{S})$, and if $q$ is not prime, then the triple $\left(\mathrm{A \Gamma L}_{m}(q) \imath\langle\tau\rangle, 2 m, q\right)$ does not satisfy Hypothesis 3.6 by Remark 3.8.

Recall that for a vector space $V$ and $H \leqslant \mathrm{GL}(V)$, we write $C_{V}(H)$ for the subspace of $V$ consisting of those vectors in $V$ that are fixed by every element of $H$.

Proposition 13.3. Let $G$ be an affine permutation group of rank 3 on $V:=V_{n}(p) \oplus V_{n}(p)$ where $n \geqslant 1, p$ is prime, $G_{0} \leqslant \mathrm{GL}_{n}(p) \lambda\langle\tau\rangle$ and $G_{0}$ is not a subgroup of $\Gamma \mathrm{L}_{1}\left(p^{n}\right) \lambda\langle\tau\rangle$. If $\mathcal{S}$ is a $G$-affine proper partial linear space in which $\mathcal{S}(0)=V_{n}(p)^{*} \times V_{n}(p)^{*}$, then $\mathcal{S}$ is isomorphic to a partial linear space $(V, \mathcal{L})$ where one of the following holds.

(i) $\mathcal{L}=\left\{\langle v\rangle_{\mathbb{F}_{q}}+w: v \in V_{n}(p)^{*} \times V_{n}(p)^{*}, w \in V\right\}$ and $G_{0} \leqslant\left(\Gamma \mathrm{L}_{m}(q) \imath\langle\tau\rangle\right) \cap \Gamma \mathrm{L}_{2 m}(q)$, where $q^{m}=p^{n}, m \geqslant 2$ and $q>2$.

(ii) $\mathcal{L}=\left\{\left\{\left(v, v^{r}\right)+w: v \in V_{n}(p)\right\}: r \in R, w \in V\right\}$ for $R \leqslant \mathrm{GL}_{n}(p)$, where $n=2$ and $(p, R)$ is one of $\left(3, Q_{8}\right),\left(5, \mathrm{SL}_{2}(3)\right),\left(7,2 \cdot S_{4}^{-}\right),\left(11, \mathrm{SL}_{2}(3) \times C_{5}\right),\left(23,2 \cdot S_{4}^{-} \times C_{11}\right),\left(11, \mathrm{SL}_{2}(5)\right)$, $\left(29, \mathrm{SL}_{2}(5) \times C_{7}\right)$ or $\left(59, \mathrm{SL}_{2}(5) \times C_{29}\right)$.

(iii) $\mathcal{L}=\left\{\left(v, v^{\alpha}\right): v \in M\right\}^{V: H_{0} \times H_{0}}$, where $p^{n}=3^{4}, M=C_{V_{4}(3)}\left(H_{0, u}\right) \simeq V_{2}(3)$ for some $u \in V_{4}(3)^{*}, \alpha \in\{1, \beta\}$ where $\beta$ is chosen to be one of the two involutions in $\operatorname{GL}(M)_{u} \backslash$ $\left(N_{\mathrm{GL}_{4}(3)}\left(H_{0}\right)\right)_{M}^{M}$, and $H_{0} \leqslant \mathrm{GL}_{4}(3)$ is one of $2 \cdot S_{5}^{-}: 2$ or $\left(D_{8} \circ Q_{8}\right) \cdot C_{5}$.

(iv) $\mathcal{L}=\left\{\left(v, v^{\alpha}\right): v \in M\right\}^{V: H_{0} \times H_{0}}$, where $p^{n}=3^{6}, M=C_{V_{6}(3)}\left(H_{0, u}\right) \simeq V_{2}(3)$ for some $u \in V_{6}(3)^{*}, \alpha \in \mathrm{GL}(M)_{u} \simeq S_{3}$ such that $\alpha^{2}=1$, and $H_{0}=\mathrm{SL}_{2}(13) \leqslant \mathrm{GL}_{6}(3)$.

(v) $\mathcal{L}=\left\{\left\{\left(v, v^{g}\right)+w: v \in V_{n}(p)\right\}: g \in \mathrm{SL}_{2}(3) \alpha \cup \mathrm{SL}_{2}(3) \alpha^{2} r, w \in V\right\}$, where $p^{n}=7^{2}, 2 \cdot S_{4}^{-}=$ $\mathrm{SL}_{2}(3)\langle r\rangle$, and $\alpha$ is chosen to be one of the non-trivial elements of $N_{\mathrm{GL}_{2}(7)}\left(2 \cdot S_{4}^{-}\right)_{u} \simeq C_{3}$ for some $u \in V_{2}(7)^{*}$.

Conversely, if one of $(i)-(v)$ holds, then $(V, \mathcal{L})$ is an affine proper partial linear space. 
We are unable to provide a classification of the $G$-affine proper partial linear spaces $\mathcal{S}$ for which $\mathcal{S}(0)=V_{n}(p)^{*} \times V_{n}(p)^{*}$ for groups $G$ in class (I0), which is why we assume that $G_{0}$ is not a subgroup of $\left.\Gamma L_{1}\left(p^{n}\right)\right\rangle\langle\tau\rangle$ in Proposition 13.3. Note that these conditions on $G$ and $\mathcal{S}$ are precisely those of Theorem 1.4(iv)(b).

Proposition 13.1 is a direct consequence of Proposition 13.5, proved below, while Proposition 13.3 will follow (with some work) from Proposition 13.20, where detailed information about the groups involved is given. See also Examples 13.12-13.17. First we consider the orbit $V_{1}^{*} \cup V_{2}^{*}$.

Lemma 13.4. Let $G$ be an affine permutation group of rank 3 on $V:=V_{n}(p) \oplus V_{n}(p)$ where $n \geqslant 1, p$ is prime and $G_{0}=G_{0, V_{1}}\langle(t, 1) \tau\rangle \leqslant \mathrm{GL}_{n}(p) \imath\langle\tau\rangle$. The following are equivalent.

(i) $\mathcal{S}$ is a $G$-affine proper partial linear space in which $\mathcal{S}(0)=V_{1}^{*} \cup V_{2}^{*}$.

(ii) $\mathcal{S}=\mathcal{S}^{\prime} \square \mathcal{S}^{\prime}$ where $\mathcal{S}^{\prime}$ is a linear space with point set $V_{n}(p)$ and line-size at least 3 admitting $V_{n}(p): G_{0}^{1}$ as a 2-transitive group of automorphisms.

Proof. Suppose that (i) holds and let $\mathcal{L}$ be the line set of $\mathcal{S}$. Let $L \in \mathcal{L}_{0}$ and $B:=L^{*}$. Without loss of generality, we may assume that $(u, 0) \in L$ for some $u \in V_{n}(p)^{*}$. If there exists $(0, v) \in B$ for some $v \in V_{n}(p)^{*}$, then $(u,-v) \in V_{1}^{*} \cup V_{2}^{*}$ by Lemma 2.15(i), a contradiction. Thus $L \subseteq V_{1}$, so $L=\left\{(v, 0): v \in L^{\prime}\right\}$ for some $L^{\prime} \subseteq V_{n}(p)$. Let $\mathcal{S}^{\prime}:=\left(V_{n}(p), \mathcal{L}^{\prime}\right)$ where $\mathcal{L}^{\prime}$ is defined to be the set of subsets $M^{\prime}$ of $V_{n}(p)$ for which $\left\{(v, 0): v \in M^{\prime}\right\} \in \mathcal{L}$. Then $\mathcal{S}^{\prime}$ is a linear space with line-size at least 3 admitting $V_{n}(p): G_{0}^{1}$ as a 2-transitive group of automorphisms. It remains to show that $\mathcal{S}=\mathcal{S}^{\prime} \square \mathcal{S}^{\prime}$. Let $M \in \mathcal{L}$. Then $M=L^{g}+\left(v_{1}, v_{2}\right)$ for some $g \in G_{0}$ and $\left(v_{1}, v_{2}\right) \in V$. Now $g=\left(g_{1}, g_{2}\right) \tau^{i}$ for some $g_{1}, g_{2} \in \mathrm{GL}_{n}(p)$ and $i \in\{1,2\}$. If $i=2$, then $M=\left\{\left(v, v_{2}\right): v \in\left(L^{\prime}\right)^{g_{1}}+v_{1}\right\}$, and if $i=1$, then $M=\left\{\left(v_{1}, v\right): v \in\left(L^{\prime}\right)^{g_{1}}+v_{2}\right\}$. Now $g_{1} \in G_{0}^{1}$ since $G_{0} \leqslant G_{0}^{1} \imath\langle\tau\rangle$, so $\left(L^{\prime}\right)^{g_{1}}+v_{j} \in \mathcal{L}^{\prime}$ for $j=1,2$. Thus $M \in \mathcal{L}^{\prime} \square \mathcal{L}^{\prime}$. On the other hand, a typical line of $\mathcal{S}^{\prime} \square \mathcal{S}^{\prime}$ has the form $\left\{(v, w): v \in M^{\prime}\right\}$ or $\left\{(w, v): v \in M^{\prime}\right\}$ for some $w \in V_{n}(p)$ and $M^{\prime} \in \mathcal{L}^{\prime}$. Now $\left\{(v, 0): v \in M^{\prime}\right\} \in \mathcal{L}$, so $\left\{(v, w): v \in M^{\prime}\right\}=\left\{(v, 0): v \in M^{\prime}\right\}+(0, w) \in \mathcal{L}$. Further, $g:=\left(1, t^{-1}\right) \tau \in G_{0}$, so $\left\{(w, v): v \in M^{\prime}\right\}=\left\{\left(v, w^{t}\right): v \in M^{\prime}\right\}^{g} \in \mathcal{L}$. Thus (ii) holds.

Conversely, suppose that (ii) holds. By Lemma $2.14, \mathcal{S}$ is a proper partial linear space with point set $V$ in which $\mathcal{S}(0)=V_{1}^{*} \cup V_{2}^{*}$, and $\operatorname{Aut}(\mathcal{S}) \geqslant\left(V_{n}(p): G_{0}^{1}\right) \curlywedge\langle\tau\rangle \simeq V:\left(G_{0}^{1} \imath\langle\tau\rangle\right) \geqslant G$. Thus (i) holds.

Using Kantor's classification [36] of the linear spaces with a 2-transitive group of automorphisms (see Theorem 2.19), we prove the following, from which Proposition 13.1 immediately follows. Note that this result includes the groups in class (I0); in fact, its proof does not require Theorem 3.3. For the following, recall the definition of $\zeta_{9}$ and $\sigma_{9}$ from $\S 2.3$.

Proposition 13.5. Let $G$ be an affine permutation group of rank 3 on $V:=V_{n}(p) \oplus V_{n}(p)$ where $n \geqslant 1, p$ is prime and $G_{0}=G_{0, V_{1}}\langle(t, 1) \tau\rangle \leqslant \mathrm{GL}_{n}(p) \imath\langle\tau\rangle$. Then $\mathcal{S}:=(V, \mathcal{L})$ is a $G$-affine proper partial linear space in which $\mathcal{S}(0)=V_{1}^{*} \cup V_{2}^{*}$ if and only if one of the following holds.

(i) $\mathcal{L}=\left\{V_{i}+v: i \in\{1,2\}, v \in V\right\}$ where $p^{n} \neq 2$.

(ii) $\mathcal{L}=\left\{\langle u\rangle_{F}+v: u \in V_{1}^{*} \cup V_{2}^{*}, v \in V\right\}$ for some subfield $F$ of $\mathbb{F}_{q}$ where $q^{m}=p^{n},|F| \geqslant 3$ and $G_{0} \leqslant \Gamma L_{m}(q) \imath\langle\tau\rangle$. If $m=1$, then $F \neq \mathbb{F}_{q}$.

(iii) $\mathcal{L}=\mathcal{L}^{\prime} \square \mathcal{L}^{\prime}$ where $\left(V_{n}(p), \mathcal{L}^{\prime}\right)$ is a linear space and one of the following holds.

(a) $G_{0} \leqslant N_{\mathrm{GL}_{4}(3)}\left(D_{8} \circ Q_{8}\right) \lambda\langle\tau\rangle$ and $p^{n}=3^{4}$, and if $\mathcal{N}$ denotes the line set of the nearfield plane of order 9 , then either $\mathcal{L}^{\prime}=\mathcal{N}$, or $\left.G_{0} \leqslant\left(\mathrm{SL}_{2}(5)\left\langle\zeta_{9} \sigma_{9}\right\rangle\right)\right\rangle\langle\tau\rangle$ where $\mathrm{SL}_{2}(5) \leqslant$ $\mathrm{SL}_{2}(9)$, in which case $\mathcal{L}^{\prime}=\mathcal{N}^{g}$ for some $g \in\left\{1, \zeta_{9}^{2}\right\}$ and $G_{0}$ is $\left(\mathrm{SL}_{2}(5)\left\langle\zeta_{9} \sigma_{9}\right)\right\rangle\langle\langle\tau\rangle$ or $\left(\mathrm{SL}_{2}(5) \times \mathrm{SL}_{2}(5)\right)\left\langle\left(\zeta_{9} \sigma_{9}, 1\right) \tau\right\rangle$.

(b) $\mathcal{L}^{\prime}$ is the line set of the Hering plane of order 27 or either of the two Hering spaces with line-size 9 , and $G_{0}=\mathrm{SL}_{2}(13) \imath\langle\tau\rangle$ where $p^{n}=3^{6}$.

Proof. Recall that $t \in G_{0}^{1}=G_{0}^{2}$ and $G_{0} \leqslant G_{0}^{1} \imath\langle\tau\rangle$. If one of (i)-(iii) holds, then $(V, \mathcal{L})$ is a $G$-affine proper partial linear space by Lemma 13.4 and Theorem 2.19.

Conversely, let $\mathcal{S}:=(V, \mathcal{L})$ be a $G$-affine proper partial linear space in which $\mathcal{S}(0)=V_{1}^{*} \cup V_{2}^{*}$. By Lemma $13.4, \mathcal{L}=\mathcal{L}^{\prime} \square \mathcal{L}^{\prime}$ where $\mathcal{S}^{\prime}:=\left(V_{n}(p), \mathcal{L}^{\prime}\right)$ is a linear space with line-size at least 3 
admitting $V_{n}(p)$ : $G_{0}^{1}$ as a 2 -transitive group of automorphisms. Thus $\mathcal{S}^{\prime}$ is given by Theorem 2.19. If Theorem 2.19(i) holds for $\mathcal{S}^{\prime}$, then $\mathcal{L}^{\prime}=\left\{V_{n}(p)\right\}$, and (i) holds.

Suppose that Theorem 2.19(ii) holds for $\mathcal{S}^{\prime}$. Then $G_{0}^{1} \leqslant \Gamma L_{m}(q)$ where $q^{m}=p^{n}$ and $\mathcal{L}^{\prime}=$ $\left\{\langle u\rangle_{F}+v: u \in V_{n}(p)^{*}, v \in V_{n}(p)\right\}$ where $F$ is a subfield of $\mathbb{F}_{q}$ with $|F| \geqslant 3$. If $m=1$ and $F=\mathbb{F}_{q}$, then (i) holds; otherwise, (ii) holds.

Suppose that Theorem 2.19(iv) holds for $\mathcal{S}^{\prime}$. Then $G_{0}^{1}=\mathrm{SL}_{2}(13)$ where $p^{n}=3^{6}$ and $\mathcal{S}^{\prime}$ is the Hering plane of order 27 or either of the two Hering spaces with line-size 9 . It is straightforward to verify that $G_{0}=\mathrm{SL}_{2}(13) 2\langle\tau\rangle$, so (iii)(b) holds.

Suppose that Theorem 2.19(iii) holds for $\mathcal{S}^{\prime}$. Now $G_{0}^{1} \leqslant N_{\mathrm{GL}_{4}(3)}\left(D_{8} \circ Q_{8}\right)$ where $p^{n}=3^{4}$. Let $\mathcal{N}$ be the line set of the nearfield plane of order 9. By Theorem 2.19, either $D_{8} \circ Q_{8} \unlhd G_{0}^{1}$ and $\mathcal{L}^{\prime}=\mathcal{N}$, or $G_{0}^{1}=\mathrm{SL}_{2}(5)\left\langle\zeta_{9} \sigma_{9}\right\rangle \leqslant \Gamma L_{2}(9)$ and $\mathcal{L}^{\prime}=\mathcal{N}^{g}$ for some $g \in\left\{1, \zeta_{9}^{2}\right\}$. In either case, $\left.G_{0} \leqslant N_{\mathrm{GL}_{4}(3)}\left(D_{8} \circ Q_{8}\right)\right\rangle\langle\tau\rangle$. If $G_{0} \leqslant\left(\mathrm{SL}_{2}(5)\left\langle\zeta_{9} \sigma_{9}\right)\right\rangle\langle\langle\tau\rangle$, then since $G$ has rank 3, it can be verified that $G_{0}=\left(\mathrm{SL}_{2}(5)\left\langle\zeta_{9} \sigma_{9}\right\rangle\right) \imath\langle\tau\rangle$ or $\left(\mathrm{SL}_{2}(5) \times \mathrm{SL}_{2}(5)\right)\left\langle\left(\zeta_{9} \sigma_{9}, 1\right) \tau\right\rangle$. Thus (iii)(a) holds.

Example 13.6. Let $\mathcal{N}$ be the nearfield plane of order 9 (see $§ 2.9$ ). By Propositions 13.1(iii)(a) or 13.5(iii)(a), $\mathcal{N} \square \mathcal{N}$ is a $G$-affine proper partial linear space with point set $V:=V_{4}(3) \oplus V_{4}(3)$ where $G:=V:\left(N_{\mathrm{GL}_{4}(3)}\left(D_{8} \circ Q_{8}\right)<\langle\tau\rangle\right)$. By Remark $2.20, N_{\mathrm{GL}_{4}(3)}\left(D_{8} \circ Q_{8}\right)=D_{8} \circ Q_{8} \circ 2 \cdot S_{5}^{-}$. Using Magma, we determine that $\operatorname{Aut}(\mathcal{N} \square \mathcal{N})=G$. Note also that the lines of $\mathcal{N} \square \mathcal{N}$ are affine subspaces of $V$.

We wish to determine the full automorphism groups of the partial linear spaces in Proposition 13.1(iii)(b), but we are unable to do this using Magma. Instead, we use the following consequence of Theorem 1.8. We will also use this result to determine the full automorphism groups of some of the partial linear spaces from Proposition 13.3.

Lemma 13.7. Let $V:=V_{n}(p) \oplus V_{n}(p)$ where $n \geqslant 2$ and $p$ is an odd prime. Let $G$ be an insoluble affine permutation group of rank 3 on $V$ where $G_{0} \leqslant \mathrm{GL}_{n}(p) \lambda\langle\tau\rangle$. Let $\mathcal{S}$ be a $G$-affine proper partial linear space with point set $V$ that is not isomorphic to the $p^{n} \times p^{n}$ grid. Then $\operatorname{Aut}(\mathcal{S})$ is an affine permutation group on $V$, and $\operatorname{Aut}(\mathcal{S})_{0} \leqslant \mathrm{GL}_{n}(p) \imath\langle\tau\rangle$.

Proof. Let $H:=\operatorname{Aut}(\mathcal{S})$. By Theorem $1.8, H$ is an affine permutation group on $V$. Since $G_{0}$ is not soluble, $H_{0}$ does not belong to class (R0). If $H_{0}$ belongs to one of the classes (T), (S) or (R1)-(R5), then $p$ divides one of the subdegrees of $H$ (see Table 4$)$, but $H$ has subdegrees $2\left(p^{n}-1\right)$ and $\left(p^{n}-1\right)^{2}$, so $p=2$, a contradiction. By Tables 2 and $3, H$ belongs to class (I), so $H_{0}$ stabilises some (internal) direct sum decomposition $V=U_{1} \oplus U_{2}$ where $U_{1}$ and $U_{2}$ are $n$-dimensional subspaces of $V$. Now $G_{0}$ also stabilises this decomposition. In particular, $V_{1}^{*} \cup V_{2}^{*}$ and $U_{1}^{*} \cup U_{2}^{*}$ are both orbits of $G_{0}$ on $V^{*}$ with order $2\left(p^{n}-1\right)$, and $2\left(p^{n}-1\right) \neq\left(p^{n}-1\right)^{2}$ since $n \geqslant 2$, so $V_{1}^{*} \cup V_{2}^{*}=U_{1}^{*} \cup U_{2}^{*}$. If $x, y \in U_{1}^{*}$ where $x \in V_{1}$ and $y \in V_{2}$, then $x+y \in U_{1}$, so without loss of generality, $x+y \in V_{1}$, but then $y \in V_{1}$, a contradiction. Thus $\left\{U_{1}, U_{2}\right\}=\left\{V_{1}, V_{2}\right\}$, so $H_{0}$ stabilises the decomposition of $V$.

Example 13.8. Let $\mathcal{H}$ be the Hering plane of order 27 or either of the two Hering spaces with line-size 9 (see §2.10). By Propositions 13.1(iii)(b) or 13.5(iii)(b), $\mathcal{H} \square \mathcal{H}$ is a $G$-affine proper partial linear space with point set $V:=V_{6}(3) \oplus V_{6}(3)$ where $G:=V:\left(\mathrm{SL}_{2}(13) \imath\langle\tau\rangle\right)$. By Lemma 13.7, $\operatorname{Aut}(\mathcal{H} \square \mathcal{H})$ is an affine permutation group of rank 3 on $V$, and $\operatorname{Aut}(\mathcal{H} \square \mathcal{H})_{0} \leqslant$ $\mathrm{GL}_{6}(3) \imath\langle\tau\rangle$. Note that $\mathrm{SL}_{2}(13)$ is not a subgroup of $\Gamma L_{3}(9)$ or $\Gamma L_{2}(27)$. Thus $\operatorname{Aut}(\mathcal{H} \square \mathcal{H})=G$ by Proposition 13.1. Using Magma, we determine that $G$ is self-normalising in $\operatorname{Sym}(V)$, so the two partial linear spaces with line-size 9 are not isomorphic. Note that the lines of $\mathcal{H} \square \mathcal{H}$ are affine subspaces of $V$ for all possible $\mathcal{H}$, and $G_{0}$ has no proper subgroups with two orbits on $V^{*}$.

Now we consider the orbit $V_{n}(p)^{*} \times V_{n}(p)^{*}$. We begin by describing a method of constructing a partial linear space on $V_{n}(p) \oplus V_{n}(p)$ from a linear space on $V_{n}(p)$ that has a 2-transitive affine group of automorphisms for which the stabiliser of any line $M$ induces a sharply 2-transitive group on $M$. There are several examples of linear spaces with such groups, including $\operatorname{AG}_{m}(q)$ 
with its group of automorphisms $\operatorname{AGL}_{m}(q)$ (where $q^{m}=p^{n}$ ), as well as $\left(V_{2}(p),\left\{V_{2}(p)\right\}\right)$ with its group of automorphisms $V_{2}(p): R$, where $R \leqslant \mathrm{GL}_{2}(p)$ and $R$ is regular on $V_{2}(p)^{*}$; for example, we may take $p=3$ and $R=Q_{8}$. The reader may find it helpful to remember these examples throughout the following exposition.

Definition 13.9. Let $\mathcal{S}:=\left(V_{n}(p), \mathcal{M}\right)$ be a linear space where $n \geqslant 1$ and $p$ is prime. The pair $\left(H_{0}, M\right)$ sharply generates $\mathcal{S}$ if the following two conditions hold.

(i) $M \in \mathcal{M}_{0}$ and $H_{0} \leqslant \mathrm{GL}_{n}(p)$.

(ii) $H:=V_{n}(p): H_{0}$ is a 2-transitive subgroup of $\operatorname{Aut}(\mathcal{S})$, and $H_{M}^{M}$ is sharply 2-transitive.

Let $R:=H_{0, M}^{M}$ and $N:=N_{\mathrm{GL}_{n}(p)}\left(H_{0}\right)$. Then $R \unlhd N_{M}^{M} \leqslant \operatorname{Sym}(M)_{0}$, so $N_{M}^{M} \leqslant N_{\mathrm{Sym}_{(M)_{0}}}(R)$. The triple $\left(H_{0}, M, \alpha\right)$ is compatible with $\mathcal{S}$ if $\left(H_{0}, M\right)$ sharply generates $\mathcal{S}$ and the following two conditions hold.

(a) $\alpha \in N_{\operatorname{Sym}(M)_{0}}(R)$ and $\alpha^{2} \in N_{M}^{M}$.

(b) There exist $w \in M^{*}$ and $h_{1}, h_{2} \in H_{0}$ such that $M^{h_{1}}+w=M$ and $\left(v^{h_{1}}+w\right)^{\alpha}=v^{\alpha h_{2}}+w^{\alpha}$ for all $v \in M$.

The triple $\left(H_{0}, M, \alpha\right)$ is $u$-compatible with $\mathcal{S}$ if $\left(H_{0}, M, \alpha\right)$ is compatible with $\mathcal{S}$ and $u^{\alpha}=u \in M^{*}$. The triple $\left(H_{0}, M, \alpha\right)$ is linearly (u-) compatible with $\mathcal{S}$ if $\left(H_{0}, M, \alpha\right)$ is $(u$-)compatible with $\mathcal{S}$, the line $M$ is a subspace of $V_{n}(p)$ (in which case $N_{M}^{M} \leqslant \mathrm{GL}\left(M, \mathbb{F}_{p}\right)$ ), and $\alpha \in \operatorname{GL}\left(M, \mathbb{F}_{p}\right)$.

Suppose that $\left(H_{0}, M\right)$ sharply generates a linear space $\mathcal{S}:=\left(V_{n}(p), \mathcal{M}\right)$. Let $H:=V_{n}(p): H_{0}$, $R:=H_{0, M}^{M}, N:=N_{\mathrm{GL}_{n}(p)}\left(H_{0}\right)$ and ${ }^{-}: N_{M} \rightarrow N_{M}^{M}$ be the natural homomorphism. Here are some important observations. First, the linear space $\mathcal{M}$ can be recovered from $\left(H_{0}, M\right)$ since $\mathcal{M}=M^{H}$. Second, if $M$ is a subspace of $V_{n}(p)$, then $H_{M}^{M}=M: R$. Lastly, for any $M$, the group $R$ is regular on $M^{*}$, so for $u \in M^{*}$, the subspace $C_{V_{n}(p)}\left(H_{0, u}\right)$ contains $M$, and if $R \unlhd X \leqslant \operatorname{Sym}(M)_{0}$, then $X=R: X_{u}$ for $u \in M^{*}$.

Here are some natural instances of triples that are compatible with $\mathcal{S}$. First, $\left(H_{0}, M, \bar{s}\right)$ is compatible with $\mathcal{S}$ for $s \in N_{M}$ : clearly (a) holds, and for any $w \in M^{*}$, there exists $h_{1} \in H_{0}$ such that $M^{h_{1}}+w=M$ since $H_{M}$ is transitive on $M$, so (b) holds with $h_{2}:=s^{-1} h_{1} s$. In particular, $\left(H_{0}, M, 1\right)$ is compatible with $\mathcal{S}$. Second, for any triple $\left(H_{0}, M, \alpha\right)$ that is compatible with $\mathcal{S}$ and any $s \in H_{0, M}$, the triple $\left(H_{0}, M, \alpha \bar{s}\right)$ is also compatible with $\mathcal{S}$ : (a) holds for $\alpha \bar{s}$ since $\alpha$ normalises $R$, and (b) holds since there exist $w \in M^{*}$ and $h_{1}, h_{2} \in H_{0}$ such that $M^{h_{1}}+w=M$ and $\left(v^{h_{1}}+w\right)^{\alpha}=v^{\alpha h_{2}}+w^{\alpha}$ for all $v \in M$, in which case $\left(v^{h_{1}}+w\right)^{\alpha \bar{s}}=v^{(\alpha \bar{s})\left(s^{-1} h_{2} s\right)}+w^{\alpha \bar{s}}$ for all $v \in M$. (However, we caution the reader that there are instances where $\left(H_{0}, M, \alpha\right)$ is a compatible triple and $s \in N_{M}$, but $(\alpha \bar{s})^{2} \notin N_{M}^{M}$ : see Examples 13.15 and 13.16.) Finally, if $M$ is a subspace of $V_{n}(p)$ and $\alpha \in N_{\mathrm{GL}\left(M, \mathbb{F}_{p}\right)}(R)$ such that $\alpha^{2} \in N_{M}^{M}$, then $\left(H_{0}, M, \alpha\right)$ is linearly compatible with $\mathcal{S}$ since (b) holds for any $w \in M^{*}$ with $h_{1}=h_{2}=1$.

Definition 13.10. Let $V:=V_{n}(p) \oplus V_{n}(p)$ where $n \geqslant 1$ and $p$ is prime, and let $\left(H_{0}, M, \alpha\right)$ be a triple that is compatible with a linear space on $V_{n}(p)$. Let $N:=N_{\mathrm{GL}_{n}(p)}\left(H_{0}\right)$ and ${ }^{-}: N_{M} \rightarrow N_{M}^{M}$ be the natural homomorphism. Recall that the stabiliser of the decomposition of $V$ in $\mathrm{GL}_{2 n}(p)$ is $\operatorname{GL}_{n}(p) \imath\langle\tau\rangle$, where $\left(u_{1}, u_{2}\right)^{\tau}=\left(u_{2}, u_{1}\right)$ for all $u_{1}, u_{2} \in V_{n}(p)$. Define

$$
\begin{aligned}
\mathcal{L}\left(H_{0}, M, \alpha\right) & :=\left\{\left(v, v^{\alpha}\right): v \in M\right\}^{V: H_{0} \times H_{0}}, \\
\mathcal{S}\left(H_{0}, M, \alpha\right) & :=\left(V, \mathcal{L}\left(H_{0}, M, \alpha\right)\right), \\
\mathcal{N}\left(H_{0}, M, \alpha\right) & :=\left\{\left(s_{1}, s_{2}\right) \in N_{M} \times N_{M}: \bar{s}_{2}=\bar{s}_{1}^{\alpha}\right\}, \\
\mathcal{G}\left(H_{0}, M, \alpha\right) & :=\left(H_{0} \times H_{0}\right) \mathcal{N}\left(H_{0}, M, \alpha\right)\left\langle\left\{(s, 1) \tau: s \in N_{M}, \bar{s}=\alpha^{2}\right\}\right\rangle .
\end{aligned}
$$

Clearly $\mathcal{N}\left(H_{0}, M, \alpha\right)$ is a group, and if $s \in N_{M}$ such that $\bar{s}=\alpha^{2}$, then $(s, 1) \tau$ normalises $\mathcal{N}\left(H_{0}, M, \alpha\right)$, so $\mathcal{N}\left(H_{0}, M, \alpha\right)\left\langle\left\{(s, 1) \tau: s \in N_{M}, \bar{s}=\alpha^{2}\right\}\right\rangle$ is a group. Thus $\mathcal{G}\left(H_{0}, M, \alpha\right)$ is a group with normal subgroups $H_{0} \times H_{0}$ and $\left(H_{0} \times H_{0}\right) \mathcal{N}\left(H_{0}, M, \alpha\right)$. Observe that for $t \in N_{M}$ such that $\bar{t}=\alpha^{2},\left(H_{0} \times H_{0}\right)\langle(t, 1) \tau\rangle$ has two orbits on $V^{*}$. In particular, $V: \mathcal{G}\left(H_{0}, M, \alpha\right)$ is a rank 3 
group. Observe also that $\mathcal{G}\left(H_{0}, M, \alpha\right)_{V_{1}}=\left(H_{0} \times H_{0}\right) \mathcal{N}\left(H_{0}, M, \alpha\right)$ since $(s, s) \in \mathcal{N}\left(H_{0}, M, \alpha\right)$ for all $s \in N_{M}$ such that $\bar{s}=\alpha^{2}$.

Here are some basic properties of $\mathcal{G}\left(H_{0}, M, \alpha\right)$ and $\mathcal{S}\left(H_{0}, M, \alpha\right)$.

Lemma 13.11. Let $V:=V_{n}(p) \oplus V_{n}(p)$ where $n \geqslant 1$ and $p$ is prime, and let $\left(H_{0}, M, \alpha\right)$ be a triple that is compatible with a linear space on $V_{n}(p)$. Let $N:=N_{\mathrm{GL}_{n}(p)}\left(H_{0}\right)$ and ${ }^{-}: N_{M} \rightarrow N_{M}^{M}$ be the natural homomorphism. Let $R:=H_{0, M}^{M}$ and $S:=N_{M}^{M}$. Let $t \in N_{M}$ be such that $\bar{t}=\alpha^{2}$.

(i) $\mathcal{N}\left(H_{0}, M, \alpha\right)\langle(t, 1) \tau\rangle=\mathcal{N}\left(H_{0}, M, \alpha\right)\left\langle\left\{(s, 1) \tau: s \in N_{M}, \bar{s}=\alpha^{2}\right\}\right\rangle$.

(ii) $\left(H_{0} \times H_{0}\right) \mathcal{N}\left(H_{0}, M, \alpha\right)=\left(H_{0} \times H_{0}\right)\left\{\left(s_{1}, s_{2}\right) \in N_{M} \times N_{M}: u^{s_{1}}=u, \bar{s}_{2}=\bar{s}_{1}^{\alpha}\right\}$ for $u \in M^{*}$.

(iii) For $s \in N_{M}, \mathcal{G}\left(H_{0}, M, \bar{s}\right)=\mathcal{G}\left(H_{0}, M, 1\right)^{(1, s)}$ and $\mathcal{L}\left(H_{0}, M, \bar{s}\right)=\mathcal{L}\left(H_{0}, M, 1\right)^{(1, s)}$.

(iv) For $r \in R, \mathcal{G}\left(H_{0}, M, \alpha r\right)=\mathcal{G}\left(H_{0}, M, \alpha\right)$ and $\mathcal{L}\left(H_{0}, M, \alpha r\right)=\mathcal{L}\left(H_{0}, M, \alpha\right)$.

(v) Suppose that $\alpha$ normalises $S$ and fixes $u \in M^{*}$. Then $\mathcal{G}\left(H_{0}, M, 1\right)=\mathcal{G}\left(H_{0}, M, \alpha\right)$ if and only if $\alpha^{2}=1$ and $\alpha$ centralises $S_{u}$.

(vi) $\mathcal{S}\left(H_{0}, M, \alpha\right)$ is a $\left(V: \mathcal{G}\left(H_{0}, M, \alpha\right)\right)$-affine partial linear space.

Proof. (i) If $s \in N_{M}$ such that $\bar{s}=\alpha^{2}$, then $\left(s t^{-1}, 1\right) \in \mathcal{N}\left(H_{0}, M, \alpha\right)$, so (i) holds.

(ii) Let $u \in M^{*}$. Recall that $R$ is regular on $M^{*}$. Let $\left(s_{1}, s_{2}\right) \in \mathcal{N}\left(H_{0}, M, \alpha\right)$. There exist $h_{1}, h_{2} \in H_{0, M}$ such that $t_{1}:=h_{1}^{-1} s_{1}$ fixes $u$ and $t_{2}:=h_{2}^{-1} s_{2}$ fixes $u^{\alpha}$, in which case $t_{1}, t_{2} \in N_{M}$. Note that $\bar{t}_{1}^{\alpha}$ and $\bar{t}_{2}$ both fix $u^{\alpha}$. Now $\bar{h}_{2} \bar{t}_{2}=\bar{s}_{2}=\bar{s}_{1}^{\alpha}=\bar{h}_{1}^{\alpha} \bar{t}_{1}^{\alpha}$, and $R_{u^{\alpha}}=1$, so $\bar{t}_{2}=\bar{t}_{1}^{\alpha}$. It follows that (ii) holds.

(iii) Let $s \in N_{M}$. Recall that $\left(H_{0}, M, \bar{s}\right)$ and $\left(H_{0}, M, 1\right)$ are compatible with $\mathcal{S}$. Since $(1, s)$ normalises $V$ and $H_{0} \times H_{0}$, it follows that $\mathcal{L}\left(H_{0}, M, 1\right)^{(1, s)}=\mathcal{L}\left(H_{0}, M, \bar{s}\right)$. It is routine to verify that $\mathcal{N}\left(H_{0}, M, 1\right)^{(1, s)}=\mathcal{N}\left(H_{0}, M, \bar{s}\right)$. Further, $((s, s) \tau)^{(1, s)}=\left(s^{2}, 1\right) \tau$ and $(s, s) \in \mathcal{N}\left(H_{0}, M, 1\right)$, so $\left(\mathcal{N}\left(H_{0}, M, 1\right)\langle\tau\rangle\right)^{(1, s)}=\mathcal{N}\left(H_{0}, M, \bar{s}\right)\left\langle\left(s^{2}, 1\right) \tau\right\rangle$. Thus (iii) holds by (i).

(iv) Let $s \in H_{0, M}$. Recall that $\left(H_{0}, M, \alpha \bar{s}\right)$ is compatible with $\mathcal{S}$. Since $(1, s) \in H_{0} \times H_{0}$, it follows that $\mathcal{L}\left(H_{0}, M, \alpha \bar{s}\right)=\mathcal{L}\left(H_{0}, M, \alpha\right)$. It is routine to verify that $\mathcal{N}\left(H_{0}, M, \alpha\right)^{(1, s)}=$ $\mathcal{N}\left(H_{0}, M, \alpha \bar{s}\right)$. Further, $(\alpha \bar{s})^{2}=\alpha^{2} \bar{s}^{\alpha} \bar{s}=\overline{t h}$ for some $h \in H_{0, M}$. Hence by (i), $\mathcal{G}\left(H_{0}, M, \alpha \bar{s}\right)=$ $\left(H_{0} \times H_{0}\right) \mathcal{N}\left(H_{0}, M, \alpha\right)^{(1, s)}\langle(t h, 1) \tau\rangle=\mathcal{G}\left(H_{0}, M, \alpha\right)$ since $(1, s),(1, h) \in H_{0} \times H_{0}$.

(v) Suppose that $\alpha$ normalises $S$ and fixes $u \in M^{*}$. First suppose that $\mathcal{G}\left(H_{0}, M, 1\right)=$ $\mathcal{G}\left(H_{0}, M, \alpha\right)$. Then $(t, 1) \tau \in \mathcal{G}\left(H_{0}, M, 1\right)$, so $(t, 1) \tau=\left(h_{1}, h_{2}\right)\left(s_{1}, s_{2}\right) \tau$ for some $h_{1}, h_{2} \in H_{0}$ and $s_{1}, s_{2} \in N_{M}$ such that $\bar{s}_{2}=\bar{s}_{1}$. Thus $h_{1}, h_{2} \in H_{0, M}$, so $\alpha^{2}=\bar{t}=\bar{h}_{1} \bar{s}_{2}=\bar{h}_{1} \bar{h}_{2}^{-1} \in R_{u}=1$. Let $\bar{t}_{1} \in S_{u}$ where $t_{1} \in N_{M}$. Now $\bar{t}_{1}^{\alpha}=\bar{t}_{2} \in S_{u}$ for some $t_{2} \in N_{M}$ since $\alpha$ normalises $S$ and fixes $u$, so $\left(t_{1}, t_{2}\right) \in \mathcal{N}\left(H_{0}, M, \alpha\right) \leqslant\left(H_{0} \times H_{0}\right) \mathcal{N}\left(H_{0}, M, 1\right)$. Since $t_{1}$ and $t_{2}$ fix $u$, and since $H_{0, u} \times H_{0, u} \leqslant \mathcal{N}\left(H_{0}, M, 1\right)$, (ii) implies that $\left(t_{1}, t_{2}\right) \in \mathcal{N}\left(H_{0}, M, 1\right)$, so $\bar{t}_{1}=\bar{t}_{2}=\bar{t}_{1}^{\alpha}$. Thus $\alpha$ centralises $S_{u}$, as desired. Conversely, if $\alpha^{2}=1$ and $\alpha$ centralises $S_{u}$, then $\bar{t}=\alpha^{2}=\overline{1}$, so $\mathcal{G}\left(H_{0}, M, \alpha\right)=\mathcal{G}\left(H_{0}, M, 1\right)$ by (i) and (ii).

(vi) Let $L:=\left\{\left(v, v^{\alpha}\right): v \in M\right\}$, and observe that $\mathcal{N}\left(H_{0}, M, \alpha\right)\langle(t, 1) \tau\rangle$ fixes $L$. By Lemma 2.7, (vi) holds if $B:=L^{*}$ is a block of $H_{0} \times H_{0}$ and $\left(V:\left(H_{0} \times H_{0}\right)\right)_{L}$ is transitive on $L$. Suppose that $\left(v, v^{\alpha}\right)^{\left(h_{1}, h_{2}\right)}=\left(w, w^{\alpha}\right)$ for some $v, w \in M^{*}$ and $\left(h_{1}, h_{2}\right) \in H_{0} \times H_{0}$. Now $h_{1}, h_{2} \in H_{0, M}$ and $v^{\alpha h_{2} \alpha^{-1}}=w=v^{h_{1}}$, and $R$ is regular on $M^{*}$, so $\alpha \bar{h}_{2}=\bar{h}_{1} \alpha$. Thus $\left(h_{1}, h_{2}\right)$ fixes $B$, so $B$ is a block of $H_{0} \times H_{0}$. By assumption, there exist $w \in M^{*}$ and $h_{1}, h_{2} \in H_{0}$ such that $M^{h_{1}}+w=M$ and $\left(v^{h_{1}}+w\right)^{\alpha}=v^{\alpha h_{2}}+w^{\alpha}$ for all $v \in M$. Let $v_{1}, v_{2} \in V_{n}(p)$ be such that $v_{1}^{h_{1}}=w$ and $v_{2}^{h_{2}}=w^{\alpha}$, and let $g:=\tau_{\left(v_{1}, v_{2}\right)}\left(h_{1}, h_{2}\right)$, where $\tau_{\left(v_{1}, v_{2}\right)}$ denotes the translation of $V$ by $\left(v_{1}, v_{2}\right)$. Now $L^{g}=L$ and $0^{g}=\left(w, w^{\alpha}\right) \in B$. It follows that $\left(V:\left(H_{0} \times H_{0}\right)\right)_{L}$ is transitive on $L$.

By Lemma 13.11(iv), since $R$ is transitive on $M^{*}$, there is no loss in assuming that $\alpha$ fixes some $u \in M^{*}$. In other words, we may restrict our attention to triples that are $u$-compatible.

It turns out that $\mathrm{AG}_{m}(q)$, the nearfield plane of order 9 and exactly one of the Hering spaces on $3^{6}$ points with line-size 9 have sharply generating pairs. Further, for $n=2$ and $p \in\{3,5,7,11,23,29,59\}$, the linear space with line set $\left\{V_{2}(p)\right\}$ has sharply generating pairs. Note that the lines of these linear spaces are all affine subspaces of $V_{n}(p)$. We now describe 
five families of examples that arise under this framework, all of which are built from linearly $u$-compatible triples.

Notation and remarks for Examples 13.12-13.16. Let $V:=V_{n}(p) \oplus V_{n}(p)$ where $n \geqslant 1$ and $p$ is prime. Recall that the stabiliser of the decomposition of $V$ is $\operatorname{GL}_{n}(p)\langle\langle\tau\rangle$, where $\left(u_{1}, u_{2}\right)^{\tau}=\left(u_{2}, u_{1}\right)$ for all $u_{1}, u_{2} \in V_{n}(p)$, and recall Definitions 13.9 and 13.10. Given a pair $\left(H_{0}, M\right)$ that sharply generates some linear space on $V_{n}(p)$ where $M$ is a subspace of $V_{n}(p)$, we let $N:=N_{\mathrm{GL}_{n}(p)}\left(H_{0}\right), R:=H_{0, M}^{M}, S:=N_{M}^{M}, T:=N_{\mathrm{GL}\left(M, \mathbb{F}_{p}\right)}(R)$ and ${ }^{-}: N_{M} \rightarrow S$ be the natural homomorphism. Recall that $R \leqslant S \leqslant T$ and $T=R: T_{u}$ for all $u \in M^{*}$. Further, $\left(H_{0}, M, \alpha\right)$ is a linearly $u$-compatible triple if and only if $\alpha \in T_{u}$ and $\alpha^{2} \in S_{u}$. Lastly, for such a triple, $\mathcal{S}\left(H_{0}, M, \alpha\right)$ is a $\left(V: \mathcal{G}\left(H_{0}, M, \alpha\right)\right)$-affine partial linear space by Lemma 13.11 (vi).

Example 13.12. Let $U:=V_{m}(q)$ where $q^{m}=p^{n}$, and let $u \in U^{*}$. Let $F$ be a subfield of $\mathbb{F}_{q}$, let $M:=\langle u\rangle_{F}$, and let $H_{0}:=\mathrm{GL}_{m}(q)$. Now $\left(U, M^{U: H_{0}}\right)$ is isomorphic to the linear space $\mathrm{AG}_{a}(r)$ where $r=|F|$ and $r^{a}=p^{n}$, and $\left(U: H_{0}\right)_{M}^{M} \simeq \operatorname{AGL}_{1}(F)$, so $\left(U: H_{0}\right)_{M}^{M}$ is sharply 2transitive. Thus $\left(H_{0}, M\right)$ sharply generates $\left(U, M^{U: H_{0}}\right)$. Further, $N=\Gamma \mathrm{L}_{m}(q), R \simeq \mathrm{GL}_{1}(F)$, and $S=T \simeq \Gamma L_{1}(F)$. Let $\alpha \in T_{u} \simeq \operatorname{Aut}(F)$. There exists $\theta \in \operatorname{Aut}(F)$ such that $(\lambda u)^{\alpha}=\lambda^{\theta} u$ for all $\lambda \in F$; with some abuse of notation, we may write $\theta$ for $\alpha$, in which case

$$
\mathcal{L}\left(\mathrm{GL}_{m}(q),\langle u\rangle_{F}, \theta\right)=\left\{\left\{\left(\lambda u_{1}+v_{1}, \lambda^{\theta} u_{2}+v_{2}\right): \lambda \in F\right\}: u_{1}, u_{2} \in U^{*}, v_{1}, v_{2} \in U\right\} .
$$

In addition, there exists $\pi \in \operatorname{Aut}\left(\mathbb{F}_{q}\right)$ such that $\left.\pi\right|_{F}=\theta^{2}$. We may assume that $\operatorname{Aut}\left(\mathbb{F}_{q}\right)$ acts on $U$ in such a way that $u$ is fixed, so by Lemma 13.11(i) and (ii), $\mathcal{G}\left(\mathrm{GL}_{m}(q),\langle u\rangle_{F}, \theta\right)$ equals

$$
\left(\operatorname{GL}_{m}(q) \times \operatorname{GL}_{m}(q)\right)\left\{\left(\sigma_{1}, \sigma_{2}\right) \in \operatorname{Aut}\left(\mathbb{F}_{q}\right) \times \operatorname{Aut}\left(\mathbb{F}_{q}\right):\left.\sigma_{1}\right|_{F}=\left.\sigma_{2}\right|_{F}\right\}\langle(\pi, 1) \tau\rangle,
$$

which is a subgroup of $\Gamma \mathrm{L}_{m}(q) \curlywedge\langle\tau\rangle$. Observe that $\mathcal{S}\left(\mathrm{GL}_{m}(q), M, 1\right) \simeq \mathcal{S}\left(\mathrm{GL}_{m}(q), M, \theta\right)$ by Lemma 13.11(iii). Further, $\mathcal{S}\left(\mathrm{GL}_{m}(q), M, 1\right)$ is an $\mathbb{F}_{q}$-dependent partial linear space that contains the line $\langle(u, u)\rangle_{F}$. In fact, if $F=\mathbb{F}_{r}$ and $r^{a}=q^{m}$, then by viewing $U$ as $V_{a}(r)$, we see that $\mathcal{L}\left(\mathrm{GL}_{m}(q), M, 1\right)=\mathcal{L}\left(\mathrm{GL}_{a}(r), M, 1\right)$ and

$$
\mathcal{G}\left(\mathrm{GL}_{m}(q), M, 1\right) \leqslant \mathcal{G}\left(\mathrm{GL}_{a}(r), M, 1\right)=\left(\Gamma \mathrm{L}_{a}(r) \imath\langle\tau\rangle\right) \cap \Gamma \mathrm{L}_{2 a}(r) .
$$

Since $\operatorname{Aut}(F) \simeq S_{u}=T_{u}$ is abelian, parts (iii) and (v) of Lemma 13.11 imply that for $\theta_{1}, \theta_{2} \in$ $\operatorname{Aut}(F)$, the group $\mathcal{G}\left(\mathrm{GL}_{m}(q), M, \theta_{1}\right)=\mathcal{G}\left(\mathrm{GL}_{m}(q), M, \theta_{2}\right)$ if and only if $\theta_{1}^{2}=\theta_{2}^{2}$, and this occurs if and only if $\theta_{2}=\theta_{1} \iota$ where $\iota \in \operatorname{Aut}(F)$ and either $\iota=1$, or, for $|\operatorname{Aut}(F)|$ even, $\iota$ is the unique involution in $\operatorname{Aut}(F)$. In particular, in this latter case, $\mathcal{G}\left(\mathrm{GL}_{m}(q), M, 1\right)$ admits both $\mathcal{S}\left(\mathrm{GL}_{m}(q), M, 1\right)$ and $\mathcal{S}\left(\mathrm{GL}_{m}(q), M, \iota\right)$ as examples.

Example 13.13. Let $M:=V_{n}(p)$ and $H_{0} \leqslant \mathrm{GL}_{n}(p)$ be regular on $V_{n}(p)^{*}$. Now $\left(H_{0}, M\right)$ sharply generates the linear space $\left(V_{n}(p),\left\{V_{n}(p)\right\}\right)$. Further, $R=H_{0}$ and $S=N=T$. Let $u \in V_{n}(p)^{*}$ and $\alpha \in T_{u}$. Since $\alpha$ normalises $R$, and since $N_{M}=N$,

$$
\begin{aligned}
& \mathcal{L}\left(H_{0}, M, \alpha\right)=\left\{\left\{\left(v+v_{1}, v^{g}+v_{2}\right): v \in V_{n}(p)\right\}: g \in R \alpha, v_{1}, v_{2} \in V_{n}(p)\right\}, \\
& \mathcal{G}\left(H_{0}, M, \alpha\right)=(R \times R)\left\{\left(s, s^{\alpha}\right): s \in T_{u}\right\}\left\langle\left(\alpha^{2}, 1\right) \tau\right\rangle .
\end{aligned}
$$

By Lemma 13.11(iii), $\mathcal{S}\left(H_{0}, M, 1\right)$ is isomorphic to $\mathcal{S}\left(H_{0}, M, \alpha\right)$ for all $\alpha \in T_{u}$. Moreover, since $S=T$, Lemma 13.11(iii) and (v) imply that for $\alpha_{1}, \alpha_{2} \in T_{u}$, the groups $\mathcal{G}\left(H_{0}, M, \alpha_{1}\right)$ and $\mathcal{G}\left(H_{0}, M, \alpha_{2}\right)$ are equal if and only if $\alpha_{1}^{2}=\alpha_{2}^{2}$ and $\alpha_{2} \alpha_{1}^{-1} \in Z\left(T_{u}\right)$.

The possibilities for $H_{0}$ are given by Zassenhaus's classification [57] of the sharply 2-transitive permutation groups (see $[20, \S 7.6]$ ); in particular, either $H_{0}$ is a subgroup of $\Gamma L_{1}\left(p^{n}\right)$, or $n=2$ and $\left(p, H_{0}, T_{u}\right)$ is given by Table 8 (cf. Theorem 2.18), in which case $\mathcal{S}\left(H_{0}, M, 1\right)$ is listed in Table 1. Note that $Q_{8} \leqslant \Gamma L_{1}(9)$, but we include this case in Table 8 since $T_{u} \nless \Gamma L_{1}(9)$. Note also that if $H_{0}=\mathrm{GL}_{1}\left(p^{n}\right)$, then $\mathcal{S}\left(H_{0}, V_{n}(p), \alpha\right)=\mathcal{S}\left(H_{0},\langle u\rangle_{F}, \alpha\right)$ where $F=\mathbb{F}_{p^{n}}$, a partial linear space from Example 13.12. For any possible $H_{0}$, the linear space $\mathcal{N}$ with point set $V$ and line set $\left\{V_{i}+v: i \in\{1,2\}, v \in V\right\} \cup \mathcal{L}\left(H_{0}, M, 1\right)$ is a nearfield plane (see $\S 2.9$ ); in particular, if 


\begin{tabular}{c|cccccccc}
\hline$p$ & 3 & 5 & 7 & 11 & 23 & 11 & 29 & 59 \\
$H_{0}$ & $Q_{8}$ & $\mathrm{SL}_{2}(3)$ & $2 \cdot S_{4}^{-}$ & $\mathrm{SL}_{2}(3) \times C_{5}$ & $2 \cdot S_{4}^{-} \times C_{11}$ & $\mathrm{SL}_{2}(5)$ & $\mathrm{SL}_{2}(5) \times C_{7}$ & $\mathrm{SL}_{2}(5) \times C_{29}$ \\
$T_{u}$ & $S_{3}$ & $C_{4}$ & $C_{3}$ & $C_{2}$ & 1 & $C_{5}$ & $C_{2}$ & 1 \\
\hline
\end{tabular}

TABLE 8. Exceptional sharply 2-transitive groups

$n=2$ and $\left(p, H_{0}\right)$ is given by Table 8 , then either $p=3$ and $\mathcal{N}$ is the nearfield plane of order 9 , or $p \neq 3$ and $\mathcal{N}$ is an irregular nearfield plane of order $p^{2}$.

We claim that for each $\left(p, H_{0}\right)$ in Table 8 , the group $V: \mathcal{G}\left(H_{0}, M, 1\right)$ is the full automorphism group of $\mathcal{S}\left(H_{0}, M, 1\right)$. If $\left(p, H_{0}\right)$ is not $\left(29, \mathrm{SL}_{2}(5) \times C_{7}\right)$ or $\left(59, \mathrm{SL}_{2}(5) \times C_{29}\right)$, then we verify this using Magma. Let $K:=\operatorname{Aut}\left(\mathcal{S}\left(H_{0}, M, 1\right)\right)$ where $\left(p, H_{0}\right)$ is $\left(29, \mathrm{SL}_{2}(5) \times C_{7}\right)$ or $\left(59, \mathrm{SL}_{2}(5) \times\right.$ $\left.C_{29}\right)$. By Lemma $13.7, K$ is an affine permutation group on $V$, and $K_{0} \leqslant \mathrm{GL}_{2}(p) \imath\langle\tau\rangle$. If $K$ belongs to (I1), then $\left\{(v, v): v \in V_{2}(p)^{*}\right\}$ is a block of $\mathrm{SL}_{2}(p) \times \mathrm{SL}_{2}(p)$, a contradiction. Thus $K$ belongs to (I4), and it follows from Lemma 13.19 below (and its proof) that $K=V: \mathcal{G}\left(H_{0}, M, 1\right)$.

Example 13.14. Here $p^{n}=3^{6}$. Let $H_{0}:=\mathrm{SL}_{2}(13) \leqslant \mathrm{GL}_{6}(3)$. Let $u \in V_{6}(3)^{*}$ and $M:=$ $C_{V_{6}(3)}\left(H_{0, u}\right) \simeq V_{2}(3)$. Using MAGMA, we determine that $M$ is a line of one of the Hering spaces $\mathcal{H}$ with line-size 9 (see $\S 2.10$ ). Now $V_{6}(3): H_{0}$ is a 2-transitive subgroup of $\operatorname{Aut}(\mathcal{H})$, so $\left(H_{0}, M\right)$ sharply generates $\mathcal{H}$. Further, $N=H_{0}, S=R=Q_{8}, T=\mathrm{GL}_{2}(3)$ and $T_{u} \simeq S_{3}$. If $\alpha \in T_{u}$ such that $\alpha^{2} \in S$, then $\alpha^{2} \in R_{u}=1$, so there are four possibilities for $\alpha$, namely 1 and the three involutions in $T_{u}$. Now $H_{0}\left\langle\langle\tau\rangle=\mathcal{G}\left(H_{0}, M, \alpha\right)\right.$ for all such $\alpha$, so $H_{0} 2\langle\tau\rangle$ admits $\mathcal{S}\left(H_{0}, M, \alpha\right)$ for all such $\alpha$. Let $K:=\operatorname{Aut}\left(\mathcal{S}\left(H_{0}, M, \alpha\right)\right)$ for such an $\alpha$. By Lemma $13.7, K$ is an affine permutation group on $V$, and $K_{0} \leqslant \mathrm{GL}_{6}(3) \imath\langle\tau\rangle$. Note that $\mathrm{SL}_{2}(13)$ is not a subgroup of $\Gamma L_{3}(9)$ or $\Gamma L_{2}(27)$. If $K$ belongs to class (I1) or (I2), then $\left\{\left(v, v^{\alpha}\right): v \in M^{*}\right\}$ is a block of $\mathrm{SL}_{6}(3) \times \mathrm{SL}_{6}(3)$ or $\operatorname{Sp}_{6}(3) \times \operatorname{Sp}_{6}(3)$, a contradiction. Thus $\operatorname{Aut}\left(\mathcal{S}\left(H_{0}, M, \alpha\right)\right)=V:\left(H_{0} \prec\langle\tau\rangle\right)$ for each $\alpha \in T_{u}$ such that $\alpha^{2}=1$. Using MAGma, we determine that $V:\left(H_{0}\langle\langle\tau\rangle)\right.$ is self-normalising in $\operatorname{Sym}(V)$, so the four partial linear spaces $\mathcal{S}\left(H_{0}, M, \alpha\right)$ are pairwise non-isomorphic.

Example 13.15. Here $p^{n}=3^{4}$. Let $U:=V_{2}(9), u \in U^{*}, \zeta:=\zeta_{9}$ and $\sigma:=\sigma_{9}$ where $u^{\sigma}=u$ (see $\S 2.3$ for the definition of $\zeta_{9}$ and $\left.\sigma_{9}\right)$. Now $\mathrm{SL}_{2}(5) \leqslant \mathrm{SL}_{2}(9)$ and $N_{\mathrm{GL}_{4}(3)}\left(\mathrm{SL}_{2}(5)\right)=\mathrm{SL}_{2}(5)\langle\zeta, \sigma\rangle$. Let $H_{0}:=\mathrm{SL}_{2}(5)\left\langle\zeta^{2}, \zeta \sigma\right\rangle \simeq 2 \cdot S_{5}^{-}: 2$ and $M:=\langle u\rangle_{\mathbb{F}_{9}}$. Note that $\mathrm{SL}_{2}(5)$ has two orbits on $U^{*}$, and if $\Omega$ is one such orbit, then $\zeta \Omega$ is the other. Now $H_{0}$ is transitive on $U^{*}$ and has order 480 . Further, $H_{0, u} \leqslant \mathrm{GL}_{2}(9)$, so $\left(H_{0}, M\right)$ sharply generates $\mathrm{AG}_{2}(9)$, and $N=\mathrm{SL}_{2}(5)\langle\zeta, \sigma\rangle$. (Note that the pair $\left(\mathrm{SL}_{2}(5)\left\langle\zeta^{i} \sigma\right\rangle, M\right)$ sharply generates $\mathrm{AG}_{2}(9)$ for $i \in\{1,3\}$, but it is more convenient to work only with $H_{0}$ since $\mathrm{SL}_{2}(5)\left\langle\zeta^{i} \sigma\right\rangle$ is not normal in $N$.) With some abuse of notation, we may write $\zeta$ for $\bar{\zeta}$ and $\sigma$ for $\bar{\sigma}$, in which case $R=\left\langle\zeta^{2}, \zeta \sigma\right\rangle=Q_{8}, S=\langle\zeta, \sigma\rangle \simeq \Gamma L_{1}(9), T=\mathrm{GL}_{2}(3)$ and $\langle\sigma\rangle=S_{u} \leqslant T_{u} \simeq S_{3}$. If $\alpha \in T_{u}$ such that $\alpha^{2} \in S_{u}$, then $\alpha^{2}=1$, so there are four possibilities for $\alpha$, namely $1, \sigma$, and the two remaining involutions in $T_{u}$. Using Lemma 13.11, we verify that $\mathcal{G}\left(H_{0}, M, 1\right)=\mathcal{G}\left(H_{0}, M, \sigma\right)=H_{0} \prec\langle\tau\rangle\langle(\sigma, \sigma)\rangle$, while for an involution $\alpha \in T_{u} \backslash\{1, \sigma\}$, $\mathcal{G}\left(H_{0}, M, \alpha\right)=H_{0} \imath\langle\tau\rangle$. In particular, any subgroup of $H_{0} \prec\langle\tau\rangle\langle(\sigma, \sigma)\rangle$ admits $\mathcal{S}\left(H_{0}, M, 1\right)$ and $\mathcal{S}\left(H_{0}, M, \sigma\right)$, while any subgroup of $H_{0}\left\langle\langle\tau\rangle\right.$ admits $\mathcal{S}\left(H_{0}, M, \alpha\right)$ for $\alpha \in T_{u}$ with $\alpha^{2}=1$. Note that $\mathcal{S}\left(H_{0}, M, 1\right) \simeq \mathcal{S}\left(H_{0}, M, \sigma\right)$ by Lemma 13.11 (iii), and $\mathcal{S}\left(H_{0}, M, \alpha\right) \simeq \mathcal{S}\left(H_{0}, M, \beta\right)$ for involutions $\alpha, \beta \in T_{u} \backslash\{1, \sigma\}$ since $\mathcal{L}\left(H_{0}, M, \alpha^{\sigma}\right)=\mathcal{L}\left(H_{0}, M, \alpha\right)^{(\sigma, \sigma)}$. By a computation in GAP using [45, 52], the full automorphism group of $\mathcal{S}\left(H_{0}, M, \alpha\right)$ is $V: \mathcal{G}\left(H_{0}, M, \alpha\right)$ for $\alpha \in T_{u}$ such that $\alpha^{2}=1$. In particular, $\mathcal{S}\left(H_{0}, M, 1\right)$ is not isomorphic to $\mathcal{S}\left(H_{0}, M, \alpha\right)$ for any involution $\alpha \in T_{u} \backslash\{1, \sigma\}$. Further, $\mathcal{S}\left(H_{0}, M, 1\right) \not \mathcal{S}\left(\mathrm{GL}_{2}(9), M, 1\right)$.

Example 13.16. Here $p^{n}=3^{4}$. Let $H_{0}:=\left(D_{8} \circ Q_{8}\right) . C_{5} \leqslant \mathrm{GL}_{4}(3)$. Let $u \in V_{4}(3)^{*}$ and $M:=C_{V_{4}(3)}\left(H_{0, u}\right) \simeq V_{2}(3)$. The nearfield plane of order 9 (see §2.9) admits the 2-transitive group $V_{4}(3): H_{0}$ as an automorphism group by Remark 2.20 and contains the line $M$, so $\left(H_{0}, M\right)$ is a sharply generating pair for this linear space. Further, $N=\left(D_{8} \circ Q_{8}\right)$. $\mathrm{AGL}_{1}(5), R=Q_{8}$, $S \simeq \Gamma L_{1}(9), T=\mathrm{GL}_{2}(3), S_{u} \simeq C_{2}, T_{u} \simeq S_{3}$ and $C_{8} \simeq N_{u} \leqslant N_{M}$ since $V_{4}(3): N$ is an automorphism group of the nearfield plane of order 9 by Remark 2.20. Write $N_{u}=\langle s\rangle$. Then 


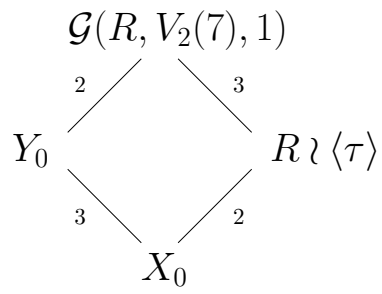

Figure 1. Groups of Example 13.17

$H_{0, u}=\left\langle s^{4}\right\rangle$ and $S_{u}=\langle\bar{s}\rangle$, so $N=H_{0}\langle s\rangle$ and $s^{2}$ fixes $M$ pointwise. Let $K_{0}:=H_{0}\left\langle s^{2}\right\rangle$, so $H_{0} \leqslant K_{0} \leqslant N$ and $K_{0}=\left(D_{8} \circ Q_{8}\right) \cdot D_{10}$. Then $K_{0, u}=\left\langle s^{2}\right\rangle$ and $M=C_{V_{4}(3)}\left(K_{0, u}\right)$, so $\left(K_{0}, M\right)$ is also a sharply generating pair for the nearfield plane of order 9 . Note that $N=N_{\mathrm{GL}_{4}(3)}\left(K_{0}\right)$. If $\alpha \in T_{u}$ such that $\alpha^{2} \in S_{u}$, then $\alpha^{2}=1$, so there are four possibilities for $\alpha$, namely $1, \bar{s}$, and the two remaining involutions in $T_{u}$. Observe that $\mathcal{L}\left(H_{0}, M, \alpha\right)=\mathcal{L}\left(K_{0}, M, \alpha\right)$ for any $\alpha \in T_{u}$ with $\alpha^{2}=1$ since $s^{2}$ fixes $M$ pointwise. Using Lemma 13.11, we verify that

$$
\mathcal{G}\left(H_{0}, M, 1\right)=\mathcal{G}\left(H_{0}, M, \bar{s}\right)=\mathcal{G}\left(K_{0}, M, 1\right)=\mathcal{G}\left(K_{0}, M, \bar{s}\right)=K_{0} \imath\langle\tau\rangle\langle(s, s)\rangle,
$$

while $\mathcal{G}\left(H_{0}, M, \alpha\right)=\mathcal{G}\left(K_{0}, M, \alpha\right)=K_{0} \prec\langle\tau\rangle$ for an involution $\alpha \in T_{u} \backslash\{1, \bar{s}\}$. In particular, any subgroup of $K_{0} \imath\langle\tau\rangle\langle(s, s)\rangle$ admits $\mathcal{S}\left(H_{0}, M, 1\right)$ and $\mathcal{S}\left(H_{0}, M, \bar{s}\right)$, while any subgroup of $K_{0} \prec\langle\tau\rangle$ admits $\mathcal{S}\left(H_{0}, M, \alpha\right)$ for $\alpha \in T_{u}$ with $\alpha^{2}=1$. Note that $\mathcal{S}\left(H_{0}, M, 1\right) \simeq \mathcal{S}\left(H_{0}, M, \bar{s}\right)$ by Lemma 13.11 (iii), and $\mathcal{S}\left(H_{0}, M, \alpha\right) \simeq \mathcal{S}\left(H_{0}, M, \beta\right)$ for involutions $\alpha, \beta \in T_{u} \backslash\{1, \bar{s}\}$ since $\mathcal{L}\left(H_{0}, M, \alpha^{\bar{s}}\right)=\mathcal{L}\left(H_{0}, M, \alpha\right)^{(s, s)}$. By a computation in GAP using [45, 52], the full automorphism group of $\mathcal{S}\left(H_{0}, M, \alpha\right)$ is $V: \mathcal{G}\left(H_{0}, M, \alpha\right)$ for $\alpha \in T_{u}$ such that $\alpha^{2}=1$. In particular, $\mathcal{S}\left(H_{0}, M, 1\right)$ is not isomorphic to $\mathcal{S}\left(H_{0}, M, \alpha\right)$ for any involution $\alpha \in T_{u} \backslash\{1, \bar{s}\}$.

Now we describe a sporadic example that is very similar to Example 13.13 but is not isomorphic to $\mathcal{S}\left(H_{0}, M, \alpha\right)$ for any $u$-compatible triple $\left(H_{0}, M, \alpha\right)$.

Example 13.17. Let $R:=2 \cdot S_{4}^{-} \leqslant \mathrm{GL}_{2}(7)$ and $T:=N_{\mathrm{GL}_{2}(7)}(R)$. Let $u \in V_{2}(7)^{*}$ and $V:=$ $V_{2}(7) \times V_{2}(7)$. Now $R$ is regular on $V_{2}(7)^{*}$, so $T=R: T_{u}$. Further, the following hold: $T_{u} \simeq C_{3}$, $R$ has a unique index 2 subgroup $K:=\mathrm{SL}_{2}(3)=R^{\prime}$, and $T=N_{\mathrm{GL}_{2}(7)}(K)$. Write $R=K\langle r\rangle$. Let $X_{0}:=(K \times K)\langle(r, 1) \tau\rangle$ and $Y_{0}:=X_{0}\left\{(s, s): s \in T_{u}\right\}$. Now $X_{0}$ has index 2 in $R \imath\langle\tau\rangle$, while $Y_{0}$ has index 2 in $\mathcal{G}\left(R, V_{2}(7), 1\right)=R \imath\langle\tau\rangle\left\{(s, s): s \in T_{u}\right\}$ (see Figure 1). For $g \in\{1, r\}$, let $\Omega(g):=\left\{\left(v, v^{h}\right): v \in V_{2}(7)^{*}, h \in K g\right\}$. Now $X_{0, V_{1}}=(K \times K)\langle(r, r)\rangle$, and the orbits of $X_{0, V_{1}}$ on $V_{2}(7)^{*} \times V_{2}(7)^{*}$ are $\Omega(1)$ and $\Omega(r)$, so $V: X_{0}$ and $V: Y_{0}$ are rank 3 groups. For $\alpha \in T_{u}$, let

$$
\mathcal{L}(\alpha):=\left\{\left\{\left(v, v^{g}\right): v \in V_{2}(7)\right\}+w: g \in K \alpha \cup K \alpha^{2} r, w \in V\right\} .
$$

Observe that $\mathcal{L}(1)=\mathcal{L}\left(R, V_{2}(7), 1\right)$ but $\mathcal{L}(\alpha) \neq \mathcal{L}\left(R, V_{2}(7), \alpha\right)$ for $\alpha \in T_{u} \backslash\{1\}$. We claim that $\mathcal{S}(\alpha):=(V, \mathcal{L}(\alpha))$ is a proper $\left(V: Y_{0}\right)$-affine partial linear space for $\alpha \in T_{u}$. Let $L_{\alpha}:=\left\{\left(v, v^{\alpha}\right)\right.$ : $\left.v \in V_{2}(7)\right\}$ and $B_{\alpha}:=L_{\alpha}^{*}$. Since $\mathcal{L}(\alpha)_{0}=L_{\alpha}^{X_{0}}$ and $\left\{(s, s): s \in T_{u}\right\}$ fixes $L_{\alpha}$, it suffices to show that $B_{\alpha}$ is a block of $X_{0}$ by Lemmas 2.3 and 2.7. Now $B_{\alpha}$ is a block of $R \times R$ and therefore of $X_{0, V_{1}}$. Recall that $(r, 1) \tau$ maps the $X_{0, V_{1}}$-orbit $\Omega(1)$ to $\Omega(r)$, and observe that $B_{\alpha} \subseteq \Omega(1)$ since $v^{\alpha} \in v^{K}$ for all $v \in V_{2}(7)^{*}$. Thus $B_{\alpha}$ is a block of $X_{0}$, and the claim holds. Note that $\mathcal{S}(\alpha)$ and $\mathcal{S}\left(\alpha^{2}\right)$ are isomorphic for $\alpha \in T_{u}$ since $\mathcal{L}\left(\alpha^{2}\right)=\mathcal{L}(\alpha)^{(1, r)}$. Let $\alpha \in T_{u} \backslash\{1\}$. Now $B_{\alpha}$ is not a block of $R \imath\langle\tau\rangle$ since $\tau$ fixes $(u, u)$ but not $B_{\alpha}$, so neither $R \imath\langle\tau\rangle$ nor $\mathcal{G}\left(R, V_{2}(7), 1\right)$ are automorphism groups of $\mathcal{S}(\alpha)$. Indeed, we verify using MAGMA that $V: Y_{0}$ is the full automorphism group of $\mathcal{S}(\alpha)$; in particular, $\mathcal{S}(\alpha)$ is not isomorphic to $\mathcal{S}(1)=\mathcal{S}\left(R, V_{2}(7), 1\right)$.

Let $\mathcal{A}(\alpha)$ be the affine plane of order 49 on $V$ with line set $\left\{V_{i}+v: i \in\{1,2\}, v \in V\right\} \cup \mathcal{L}(\alpha)$ for $\alpha \in T_{u}$. By [43, Theorem 19.10], there are (up to isomorphism) two affine planes of order 49 of type $\mathrm{F} * 7$ (as defined in [43, p.95]): the irregular nearfield plane $\mathcal{A}(1)$ (cf. Example 13.13) and the exceptional Lüneburg plane of order 49 . We claim that $\mathcal{A}(\alpha)$ is isomorphic to the latter when 
$\alpha \neq 1$. Now $\mathcal{A}(\alpha) \not \mathcal{A}(1)$ when $\alpha \neq 1$, or else $\mathcal{S}(\alpha) \simeq \mathcal{S}(1)$ by Remark 2.12, a contradiction. Thus it suffices to show that $\mathcal{A}(\alpha)$ is of type $\mathrm{F} * 7$. To do so, we must show that $\mathcal{A}(\alpha)$ has properties (j) and (ij) of [43, p.95]. Property (j) requires Aut $(\mathcal{A}(\alpha))_{L}$ to be 2 -transitive on $L$ for all lines $L$, which holds by Lemma 2.6(ii). For (ij), let $P$ and $Q$ be the points at infinity of the lines $V_{1}$ and $V_{2}$, respectively, and let $O$ be the point $0 \in V$. Let $S_{0}:=K \times 1$ and $S_{\infty}:=1 \times K$. Then $S_{0} \simeq S_{\infty} \simeq \mathrm{SL}_{2}(3)$, and $S_{0}$ and $S_{\infty}$ have the same four orbits on $\ell_{\infty}$. Further, $S_{0}$ fixes $P$ and the line on $O$ and $Q$ pointwise (i.e., $V_{2} \cup\{Q\}$ ), while $S_{\infty}$ fixes $Q$ and the line on $O$ and $P$ pointwise (i.e., $V_{1} \cup\{P\}$ ). Since (ij) requires the existence of $P, Q, O, S_{0}$ and $S_{\infty}$ with precisely these properties, $\mathcal{A}(\alpha)$ is of type $\mathrm{F} * 7$, and the claim holds.

We wish to prove that no other examples arise; the following lemma is a basic but crucial observation.

Lemma 13.18. Let $G$ be an affine permutation group of rank 3 on $V:=V_{n}(p) \oplus V_{n}(p)$ where $n \geqslant 1, p$ is prime and $G_{0} \leqslant \mathrm{GL}_{n}(p) \imath\langle\tau\rangle$. Let $\mathcal{S}:=(V, \mathcal{L})$ be a $G$-affine proper partial linear space in which $\mathcal{S}(0)=V_{n}(p)^{*} \times V_{n}(p)^{*}$. Let $L \in \mathcal{L}_{0}$. Then $L=\left\{\left(v, v^{\alpha}\right): v \in M\right\}$ for some $M \subseteq V_{n}(p)$ and injective map $\alpha: M \rightarrow V_{n}(p)$. In particular, if $(x, x) \in L$, then $\alpha$ fixes $x$.

Proof. If $\left(v, w_{1}\right),\left(v, w_{2}\right) \in L^{*}$ for some $v, w_{1}, w_{2} \in V_{n}(p)$, then $w_{1}=w_{2}$ by Lemma 2.15(i). The desired result then follows from the symmetry of this argument.

In the following, we prove that if $H_{0} \times H_{0} \unlhd G_{0}$ where $H_{0}$ is transitive on $V_{n}(p)^{*}$, then every $G$-affine proper partial linear space $\mathcal{S}$ with $\mathcal{S}(0)=V_{n}(p)^{*} \times V_{n}(p)^{*}$ has the form $\mathcal{S}\left(H_{0}, M, \alpha\right)$ for some $u$-compatible triple $\left(H_{0}, M, \alpha\right)$. In order to give an elementary proof of this fact, we do not assume that the triple is linearly compatible. Note that if $H_{0} \times H_{0} \leqslant G_{0}=G_{0, V_{1}}\langle(t, 1) \tau\rangle \leqslant$ $\mathrm{GL}_{n}(p) \imath\langle\tau\rangle$ where $H_{0} \leqslant \mathrm{GL}_{n}(p)$ is transitive on $V_{n}(p)^{*}$, then for any $u \in V_{n}(p)^{*}$, there exists $h \in H_{0}$ such that $u^{h t}=u$, and $G_{0}=G_{0, V_{1}}\langle(h t, 1) \tau\rangle$. Thus, for such $G_{0}$, there is no loss of generality in assuming that $t$ fixes some non-zero vector.

Lemma 13.19. Let $G$ be an affine permutation group of rank 3 on $V:=V_{n}(p) \oplus V_{n}(p)$ where $n \geqslant 1, p$ is prime and $H_{0} \times H_{0} \unlhd G_{0}=G_{0, V_{1}}\langle(t, 1) \tau\rangle \leqslant \mathrm{GL}_{n}(p) \imath\langle\tau\rangle$ such that $H_{0} \leqslant \mathrm{GL}_{n}(p)$ is transitive on $V_{n}(p)^{*}$ and $t \in \mathrm{GL}_{n}(p)_{u}$ for some $u \in V_{n}(p)^{*}$. The following are equivalent.

(i) $\mathcal{S}$ is a $G$-affine proper partial linear space in which $\mathcal{S}(0)=V_{n}(p)^{*} \times V_{n}(p)^{*}$.

(ii) $\mathcal{S}=\mathcal{S}\left(H_{0}, M, \alpha\right)$ and $G_{0} \leqslant \mathcal{G}\left(H_{0}, M, \alpha\right)$ for some triple $\left(H_{0}, M, \alpha\right)$ that is u-compatible with a linear space on $V_{n}(p)$ such that $|M| \geqslant 3$ and $\alpha^{2}=\left.t\right|_{M}$.

Proof. If (ii) holds, then (i) holds by Lemma 13.11(vi). Conversely, suppose that (i) holds, and let $\mathcal{L}$ be the line set of $\mathcal{S}$. Recall that $t \in G_{0}^{1}=G_{0}^{2}$, and note that $G_{0}^{1} \leqslant N:=N_{\mathrm{GL}_{n}(p)}\left(H_{0}\right)$ since $H_{0} \unlhd G_{0}^{1}$. Let $L \in \mathcal{L}_{0}$ be such that $(u, u) \in L$. By Lemma 13.18, $L=\left\{\left(v, v^{\alpha}\right): v \in M\right\}$ for some $M \subseteq V_{n}(p)$ and injective map $\alpha: M \rightarrow V_{n}(p)$, where $\alpha$ fixes 0 and $u$. Also, $|M|=|L| \geqslant 3$. Let - $: N_{M} \rightarrow N_{M}^{M}$ be the natural homomorphism and $R:=H_{0, M}^{M}$. Let $B:=L^{*}$. By Lemma $2.6, B$ is a block of $G_{0}$. Then $(t, 1) \tau \in G_{0, B}$ since $(t, 1) \tau$ fixes $(u, u)$. Thus $L=\left\{\left(v^{\alpha}, v^{t}\right): v \in M\right\}$, so $\alpha \in \operatorname{Sym}(M)$ and $t \in N_{M}$. Further $\alpha^{2}=\bar{t}=\left.t\right|_{M}$.

Let $H:=V_{n}(p): H_{0}$. We claim that $\left(V_{n}(p), M^{H}\right)$ is a linear space. By Lemma 2.7, it suffices to show that $C:=M^{*}$ is a block of $H_{0}$ on $V_{n}(p)^{*}$ and that $H_{M}$ is transitive on $M$. Now $C$ is a block of $H_{0}$ on $V_{n}(p)^{*}$, for if $v^{h}=w$ for some $v, w \in C$ and $h \in H_{0}$, then there exists $h^{\prime} \in H_{0}$ such that $\left(v^{\alpha}\right)^{h^{\prime}}=w^{\alpha}$, so $\left(h, h^{\prime}\right) \in G_{0, B}$, in which case $C^{h}=C$, as desired. Recall that $(t, 1) \tau$ fixes $(u, u)$ and $L$, and note that $V:\left(H_{0} \times H_{0}\right)\langle(t, 1) \tau\rangle$ is a rank 3 subgroup of $G$. Now, by Lemma 2.6, there exist $\left(v_{1}, v_{2}\right) \in V$ and $\left(h_{1}, h_{2}\right) \in H_{0} \times H_{0}$ such that $g:=\tau_{\left(v_{1}, v_{2}\right)}\left(h_{1}, h_{2}\right)$ fixes $L$ and maps $(0,0)$ to $(u, u)$, where $\tau_{\left(v_{1}, v_{2}\right)}$ denotes the translation of $V$ by $\left(v_{1}, v_{2}\right)$. Then $L=L^{g}=\left\{\left(v^{h_{1}}+u, v^{\alpha h_{2}}+u\right): v \in M\right\}$. In particular, $\tau_{v_{1}} h_{1}$ fixes $M$ and maps 0 to $u$, where $\tau_{v_{1}}$ denotes the translation of $V_{n}(p)$ by $v_{1}$. Thus $H_{M}$ is transitive on $M$, and the claim holds.

Next we claim that that $H_{M}^{M}$ is sharply 2-transitive, in which case $\left(H_{0}, M\right)$ sharply generates $\left(V_{n}(p), M^{H}\right)$. Since $H_{M}^{M}$ is 2 -transitive, it suffices to prove that $H_{0, u}$ fixes $C=M^{*}$ pointwise. 
Let $h \in H_{0, u}$. Now $(h, 1) \in G_{0, B}$, so $\left(v^{h}, v^{\alpha}\right) \in B$ for all $v \in C$, in which case $\left(v^{h}\right)^{\alpha}=v^{\alpha}$ for all $v \in C$. Since $\alpha$ is injective, $v^{h}=v$ for all $v \in C$, as desired.

Now we claim that $\left(H_{0}, M, \alpha\right)$ is $u$-compatible with $\left(V_{n}(p), M^{H}\right)$. For condition (a) of Definition 13.9, since $\alpha^{2}=\bar{t} \in N_{M}^{M}$, it remains to prove that $\alpha$ normalises $R$. Let $h \in H_{0, M}$. There exists $h^{\prime} \in H_{0, M}$ such that $u^{h^{\prime}}=\left(u^{h}\right)^{\alpha}$. Now $\left(h, h^{\prime}\right) \in G_{0, B}$, so $L=\left\{\left(v^{h}, v^{\alpha h^{\prime}}\right): v \in M\right\}$, in which case $\bar{h} \alpha=\alpha \overline{h^{\prime}}$. Thus $\alpha$ normalises $R$. We saw above that there exist $h_{1}, h_{2} \in H_{0}$ such that $L=\left\{\left(v^{h_{1}}+u, v^{\alpha h_{2}}+u\right): v \in M\right\}$. In particular, $M^{h_{1}}+u=M$ and $\left(v^{h_{1}}+u\right)^{\alpha}=v^{\alpha h_{2}}+u^{\alpha}$ for all $v \in M$, so condition (b) of Definition 13.9 holds, and the claim follows.

It remains to prove that $G_{0} \leqslant \mathcal{G}\left(H_{0}, M, \alpha\right)$ and $\mathcal{L}=\mathcal{L}\left(H_{0}, M, \alpha\right)$. By definition, $\mathcal{L}\left(H_{0}, M, \alpha\right) \subseteq$ $L^{G}=\mathcal{L}$, so it suffices to show that $G_{0} \leqslant \mathcal{G}\left(H_{0}, M, \alpha\right)$. Since $\bar{t}=\alpha^{2}$ where $t \in N_{M}$ and $G_{0}=$ $G_{0, V_{1}}\langle(t, 1) \tau\rangle$, we only need to show that $G_{0, V_{1}} \leqslant\left(H_{0} \times H_{0}\right) \mathcal{N}\left(H_{0}, M, \alpha\right)$. Let $\left(g_{1}, g_{2}\right) \in G_{0, V_{1}}$. There exist $h_{1}, h_{2} \in H_{0}$ such that $u^{h_{1} g_{1}}=u=u^{h_{2} g_{2}}$. Let $s_{1}:=h_{1} g_{1}$ and $s_{2}:=h_{2} g_{2}$. Now $\left(s_{1}, s_{2}\right) \in G_{0, B}$, so $L=\left\{\left(v^{s_{1}}, v^{\alpha s_{2}}\right): v \in M\right\}$, and $s_{1}, s_{2} \in N_{M}$ since $H_{0} \unlhd G_{0}^{1} \leqslant N$. Further, $\bar{s}_{1} \alpha=\alpha \bar{s}_{2}$, so $\bar{s}_{2}=\bar{s}_{1}^{\alpha}$. Thus $\left(g_{1}, g_{2}\right)=\left(h_{1}^{-1}, h_{2}^{-1}\right)\left(s_{1}, s_{2}\right) \in\left(H_{0} \times H_{0}\right) \mathcal{N}\left(H_{0}, M, \alpha\right)$.

Now we prove that Examples 13.12-13.17 are the only partial linear spaces $\mathcal{S}$ on $V_{n}(p) \times V_{n}(p)$ with an affine automorphism group in class (I) but not (I0) for which $\mathcal{S}(0)=V_{n}(p)^{*} \times V_{n}(p)^{*}$. In particular, we see that with the exception of Example 13.17, every such partial linear space has the form of Definition 13.10. We also provide more information about the possible rank 3 groups that arise. Although the groups $\mathcal{G}\left(H_{0}, M, 1\right)$ and $\mathcal{G}\left(H_{0}, M, \alpha\right)$ are often conjugate, we work with arbitrary $\alpha$ since this is not true in general (see Examples 13.15 and 13.16). For the following, recall the definition of $\zeta_{q}$ and $\sigma_{q}$ from $\S 2.3$.

Proposition 13.20. Let $G$ be an affine permutation group of rank 3 on $V:=V_{n}(p) \oplus V_{n}(p)$ where $n \geqslant 2, p$ is prime, $G_{0}=G_{0, V_{1}}\langle(t, 1) \tau\rangle \leqslant \mathrm{GL}_{n}(p) 2\langle\tau\rangle$ and $G_{0}$ is not a subgroup of $\Gamma L_{1}\left(p^{n}\right) \curlywedge\langle\tau\rangle$. Let $u \in V_{n}(p)^{*}$. Then $\mathcal{S}:=(V, \mathcal{L})$ is a $G$-affine proper partial linear space in which $\mathcal{S}(0)=V_{n}(p)^{*} \times V_{n}(p)^{*}$ if and only if one of the following holds (up to conjugacy in $\left.\mathrm{GL}_{n}(p) \imath\langle\tau\rangle\right)$.

(i) $\mathcal{L}=\mathcal{L}\left(H_{0}, M, \alpha\right)$ and $G_{0} \leqslant \mathcal{G}\left(H_{0}, M, \alpha\right)$ for some triple $\left(H_{0}, M, \alpha\right)$ that is linearly $u$ compatible with a linear space on $V_{n}(p)$. Here $\alpha \in T_{u}$ where $T:=N_{\mathrm{GL}\left(M, \mathbb{F}_{p}\right)}\left(H_{0, M}^{M}\right)$, and one of the following holds.

(a) $H_{0}=\mathrm{GL}_{m}(q)$ and $M=\langle u\rangle_{F}$ for some subfield $F$ of $\mathbb{F}_{q}$ where $q^{m}=p^{n}, m \geqslant 2$ and $|F| \geqslant 3$. Here there exists $\theta \in \operatorname{Aut}(F)$ such that $(\lambda u)^{\alpha}=\lambda^{\theta} u$ for all $\lambda \in F$, and $\theta^{2}=\left.\pi\right|_{F}$ where $\pi \in \operatorname{Aut}\left(\mathbb{F}_{q}\right)$ is such that $t$ is $\pi$-semilinear.

(b) $H_{0}$ is regular on $V_{n}(p)^{*}$ and $M=V_{n}(p)$ where $n=2$ and $\left(p, H_{0}, T_{u}\right)$ is given by Table 8. Either $H_{0} \times H_{0} \unlhd G_{0}$ and $\alpha^{2} t^{-1} \in H_{0}$, or one of the following holds.

(1) $p=7$ and either $G_{0}=X_{0}$ and $\alpha=1$, or $G_{0}=X_{0}^{g_{\alpha}}\left\{(s, s): s \in T_{u}\right\}$ where $g_{\alpha}=(1, \alpha), X_{0}=\left(\mathrm{SL}_{2}(3) \times \mathrm{SL}_{2}(3)\right)\langle(r, 1) \tau\rangle$ and $H_{0}=2 \cdot S_{4}^{-}=\mathrm{SL}_{2}(3)\langle r\rangle$.

(2) $p=23$ and $G_{0}=(K \times K)\langle(t, 1) \tau\rangle$ where $K:=\mathrm{SL}_{2}(3) \times C_{11}$ is the unique index 2 subgroup of $H_{0}=T=2 \cdot S_{4}^{-} \times C_{11}$, and $t \in H_{0} \backslash K$.

(c) $H_{0}=\mathrm{SL}_{2}(13)$ and $M=C_{V_{6}(3)}\left(H_{0, u}\right) \simeq V_{2}(3)$ where $p^{n}=3^{6}$. Here $T_{u} \simeq S_{3}, \alpha^{2}=1$ and $G_{0}=\mathrm{SL}_{2}(13) \imath\langle\tau\rangle=\mathcal{G}\left(H_{0}, M, \alpha\right)$.

(d) $H_{0}=\mathrm{SL}_{2}(5)\left\langle\zeta_{9}^{2}, \zeta_{9} \sigma_{9}\right\rangle \leqslant \Gamma L_{2}(9)$ and $M=\langle u\rangle_{\mathbb{F}_{9}}$ where $p^{n}=3^{4}$ and $u^{\sigma 9}=u$. Here $T_{u} \simeq S_{3}, \alpha^{2}=1, \mathrm{SL}_{2}(5) \times \mathrm{SL}_{2}(5) \unlhd G_{0} \leqslant H_{0} \imath\langle\tau\rangle\left\langle\left(\sigma_{9}, \sigma_{9}\right)\right\rangle=\mathcal{G}\left(H_{0}, M, 1\right)$, and if $G_{0} \nless H_{0} 2\langle\tau\rangle$, then $\alpha \in\left\{1, \sigma_{9}\right\}$.

(e) $H_{0}=\left(D_{8} \circ Q_{8}\right) \cdot C_{5}$ and $M=C_{V_{4}(3)}\left(H_{0, u}\right) \simeq V_{2}(3)$ where $p^{n}=3^{4}$. Here $T_{u} \simeq S_{3}$, $\alpha^{2}=1, H_{0} \times H_{0} \unlhd G_{0} \leqslant\left(H_{0}\left\langle s^{2}\right\rangle\right) \imath\langle\tau\rangle\langle(s, s)\rangle=\mathcal{G}\left(H_{0}, M, 1\right), H_{0}\langle s\rangle=N_{\mathrm{GL}_{4}(3)}\left(H_{0}\right)$, $u^{s}=u, M^{s}=M$, and if $G_{0} \nless\left(H_{0}\left\langle s^{2}\right\rangle\right) \imath\langle\tau\rangle$, then $\alpha \in\left\{1,\left.s\right|_{M}\right\}$.

(ii) $\mathcal{L}=\left\{\left\{\left(v, v^{g}\right)+w: v \in V_{n}(p)\right\}: g \in \mathrm{SL}_{2}(3) \alpha \cup \mathrm{SL}_{2}(3) \alpha^{2} r, w \in V\right\}$ and $G_{0}=X_{0}$ or $X_{0}\left\{(s, s): s \in T_{u}\right\}$, where $p^{n}=7^{2}, T=N_{\mathrm{GL}_{n}(p)}\left(2 \cdot S_{4}^{-}\right), X_{0}=\left(\mathrm{SL}_{2}(3) \times \mathrm{SL}_{2}(3)\right)\langle(r, 1) \tau\rangle$, $2 \cdot S_{4}^{-}=\mathrm{SL}_{2}(3)\langle r\rangle, \alpha \in T_{u} \backslash\{1\}$ and $T_{u} \simeq C_{3}$. 
Proof. If (i) holds, then $\mathcal{S}$ is a $G$-affine proper partial linear space by Lemma 13.11 and the information given in Examples 13.12-13.16. If (ii) holds, then $\mathcal{S}$ is a $G$-affine proper partial linear space by the information given in Example 13.17.

Conversely, suppose that $\mathcal{S}:=(V, \mathcal{L})$ is a $G$-affine proper partial linear space in which $\mathcal{S}(0)=$ $V_{n}(p)^{*} \times V_{n}(p)^{*}$. There exists $L \in \mathcal{L}_{0}$ such that $(u, u) \in L$. Let $B:=L^{*}$. By Lemma 2.6, $B$ is a block of $G_{0}$ on $V_{n}(p)^{*} \times V_{n}(p)^{*}$. By Lemma 13.18, $L=\left\{\left(v, v^{\alpha}\right): v \in M\right\}$ for some $M \subseteq V_{n}(p)$ with $|M| \geqslant 3$ and injective map $\alpha: M \rightarrow V_{n}(p)$, where $\alpha$ fixes 0 and $u$. By assumption, $G_{0}$ belongs to one of the classes (I1)-(I8), so that (in the notation of Theorem 3.1), $G_{0} \leqslant \Gamma \mathrm{L}_{m}(q) \imath\langle\tau\rangle$ where $q^{m}=p^{n}$ and $m \geqslant 2$. Recall that $t \in G_{0}^{1}=G_{0}^{2} \leqslant \Gamma \mathrm{L}_{m}(q)$. Let $\sigma:=\sigma_{q}$. We may assume without loss of generality that $u^{\sigma}=u$. Let $\zeta:=\zeta_{q}$. Let $\pi \in \operatorname{Aut}\left(\mathbb{F}_{q}\right)$ be such that $t$ is $\pi$-semilinear.

Assume for this paragraph that $H_{0} \times H_{0} \unlhd G_{0}$ where $H_{0} \leqslant \Gamma \mathrm{L}_{m}(q)$ is transitive on $V_{n}(p)^{*}$. Now $u^{h t}=u$ for some $h \in H_{0}$, so by Lemma 13.19, $\mathcal{L}=\mathcal{L}\left(H_{0}, M, \alpha\right), G_{0} \leqslant \mathcal{G}\left(H_{0}, M, \alpha\right)$ and $\left(H_{0}, M, \alpha\right)$ is $u$-compatible with a linear space on $V_{n}(p)$. In particular, $M \subseteq C_{V_{n}(p)}\left(H_{0, u}\right)$. Further, $\alpha^{2}=\overline{h t}$ and $h t \in N_{M}$, where $N:=N_{\mathrm{GL}_{n}(p)}\left(H_{0}\right)$ and ${ }^{-}: N_{M} \rightarrow N_{M}^{M}$ is the natural homomorphism. Note that the line $M$ is described by Theorem 2.19, and $H_{0}$ is regular on $V_{n}(p)^{*}$ when $M=V_{n}(p)$. Note also that if $L$ is an $\mathbb{F}_{p}$-subspace of $V$, then $M$ is an $\mathbb{F}_{p}$-subspace of $V_{n}(p)$ and $\alpha \in \mathrm{GL}\left(M, \mathbb{F}_{p}\right)$, in which case $\alpha \in T_{u}$ where $T:=N_{\mathrm{GL}\left(M, \mathbb{F}_{p}\right)}\left(H_{0, M}^{M}\right)$ and $\left(H_{0}, M, \alpha\right)$ is linearly $u$-compatible. Suppose now that $H_{0} \leqslant \mathrm{GL}_{m}(q)$ and $M=\langle u\rangle_{F}$ for some subfield $F$ of $\mathbb{F}_{q}$. We claim that (i)(a) holds. Note that $H_{0, M}^{M}=\mathrm{GL}_{m}(q)_{M}^{M} \simeq \mathrm{GL}_{1}(F)$. There exists $g \in H_{0}$ such that $u^{g}=-u$. Now $L \subseteq M \oplus M$ since $\alpha \in \operatorname{Sym}(M)$, and $g \in \mathrm{GL}_{m}(q)$, so $L$ is an $\mathbb{F}_{p}$-subspace of $V$ by Lemma 2.16. Thus $\alpha \in T_{u} \simeq \operatorname{Aut}(F)$, and we have already seen that $\alpha^{2}=\overline{h t} \in \Gamma \mathrm{L}_{m}(q)_{M}^{M}$, so $\left(\mathrm{GL}_{m}(q), M, \alpha\right)$ is linearly $u$-compatible. Further, there exists $\theta \in \operatorname{Aut}(F)$ such that $(\lambda u)^{\alpha}=\lambda^{\theta} u$ for all $\lambda \in F$, and $\theta^{2}=\left.\pi\right|_{F}$. Now $L^{\mathrm{GL}_{m}(q) \times \mathrm{GL}_{m}(q)}=L^{H_{0} \times H_{0}}$, so $\mathcal{L}\left(\mathrm{GL}_{m}(q), M, \alpha\right)=\mathcal{L}\left(H_{0}, M, \alpha\right)=\mathcal{L}$. Recall that $G_{0}=G_{0, V_{1}}\langle(h t, 1) \tau\rangle$, and $G_{0, V_{1}} \leqslant\left(H_{0} \times\right.$ $\left.H_{0}\right)\left\{\left(s_{1}, s_{2}\right) \in N_{M} \times N_{M}: \bar{s}_{2}=\bar{s}_{1}^{\alpha}\right\}$. Since $G_{0, V_{1}} \leqslant \Gamma \mathrm{L}_{m}(q) \times \Gamma \mathrm{L}_{m}(q)$ and $h t$ is $\pi$-semilinear, it follows that $G_{0} \leqslant \mathcal{G}\left(\mathrm{GL}_{m}(q), M, \alpha\right)$ (cf. Example 13.12), and the claim holds.

We now consider the various possibilities for $G_{0}$. Whenever $H_{0} \times H_{0} \unlhd G_{0}$ such that $H_{0} \leqslant$ $\Gamma \mathrm{L}_{m}(q)$ is transitive on $V_{n}(p)^{*}$, we make use of the above observations and notation.

Suppose that $G_{0}$ belongs to one of (I1)-(I3). We may take $H_{0}$ to be $\mathrm{SL}_{m}(q), \mathrm{Sp}_{m}(q)$ or $G_{2}(q)^{\prime}$, respectively (by [55, p.125] when $\left.H_{0}=G_{2}(q)^{\prime}\right)$. Now $H_{0}$ is not regular on $V_{n}(p)^{*}$, so $M \neq V_{n}(p)$, in which case $M=\langle u\rangle_{F}$ for some subfield $F$ of $\mathbb{F}_{q}$ by Theorem 2.19. Thus (i)(a) holds.

Suppose that $G_{0}$ belongs to (I5). We may take $H_{0}$ to be $A_{6}$ or $A_{7}$. Now $H_{0}$ is not regular on $V_{n}(p)^{*}$, so $M \neq V_{n}(p)$, but this contradicts Theorem 2.19.

Suppose that $G_{0}$ belongs to (I6). We may take $H_{0}$ to be $\mathrm{SL}_{2}(13)$, which is self-normalising in $\mathrm{GL}_{6}(3)$. Since $-1 \in H_{0}$, Lemma 2.16 implies that $L$ is a subspace of $V_{6}(3)$, so $M$ is a subspace of $C_{V_{6}(3)}\left(\mathrm{SL}_{2}(13)_{u}\right) \simeq V_{2}(3)$. Either $M=\langle u\rangle_{\mathbb{F}_{3}}$, in which case (i)(a) holds, or $M=$ $C_{V_{6}(3)}\left(\mathrm{SL}_{2}(13)_{u}\right)$, in which case (i)(c) holds by Example 13.14 .

Suppose that $G_{0}$ belongs to (I7), and let $N^{*}:=N_{\mathrm{GL}_{m}(q)}\left(\mathrm{SL}_{2}(3)\right)$. Then $G_{0} \leqslant N^{*} \imath\langle\tau\rangle$. Note that $m=2$ and $q=p$. Using MAGMA, we determine that $-1 \in G_{0}$, so $L$ is an $\mathbb{F}_{p}$-subspace of $V$ by Lemma 2.16. In particular, $M$ is an $\mathbb{F}_{p}$-subspace of $V_{2}(p)$ and $|L|$ divides $p^{2}$. If $|L|=p$, then $L=\langle(u, u)\rangle_{\mathbb{F}_{p}}$ and $G_{0} \leqslant \mathrm{GL}_{2}(p) \imath \tau=\mathcal{G}\left(\mathrm{GL}_{2}(p),\langle u\rangle_{\mathbb{F}_{p}}, 1\right)$, so (i)(a) holds. Otherwise, $|L|=p^{2}$ and $M=V_{2}(p)$. Using MAGMA, we determine that $N^{*}$ has a subgroup $R$ that is regular on $V_{2}(p)^{*}$ and isomorphic to a group in Table 8. Moreover, $N^{*}=N_{\mathrm{GL}_{2}(p)}(R)$, so $G_{0}$ normalises $R \times R$. If $R \times R \subseteq G_{0}$, then we may take $H_{0}$ to be $R$, in which case $\alpha^{2} t^{-1}=h \in H_{0}$ and $T_{u}$ is given by Table 8 , so (i)(b) holds. Suppose instead that $R \times R$ is not a subgroup of $G$. Since $G_{0} \nless \Gamma L_{1}\left(p^{2}\right) \imath\langle\tau\rangle$, it can be checked using MAGMA that either $p=23$ and (i)(b)(2) holds, or $p=7$, in which case either (i)(b)(1) holds, or (ii) holds (up to conjugacy in $N^{*} \times N^{*}$ ).

Suppose that $G_{0}$ belongs to (I4). Then $S \times S \unlhd G_{0} \leqslant N^{*} \imath\langle\tau\rangle$ where $S:=\mathrm{SL}_{2}(5), N^{*}:=$

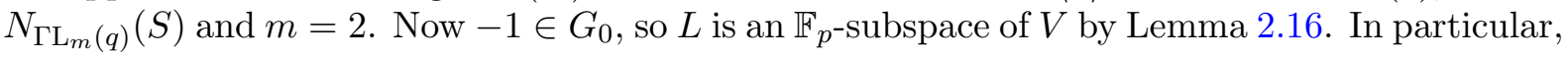


$M$ is an $\mathbb{F}_{p}$-subspace of $V_{n}(p)$ and $|L|$ divides $q^{2}$. Suppose that $q \in\{11,19,29,59\}$ (we treat the case $q=9$ below). Now $q=p$, so if $|L|=p$, then $L=\langle(u, u)\rangle_{\mathbb{F}_{p}}$ and $G_{0} \leqslant \mathrm{GL}_{2}(p) \imath \tau=$ $\mathcal{G}\left(\mathrm{GL}_{2}(p),\langle u\rangle_{\mathbb{F}_{p}}, 1\right)$, so (i)(a) holds. Otherwise, $|L|=p^{2}$ and $M=V_{2}(p)$. By a computation in Magma, $N^{*}=S\langle\zeta\rangle$, so $\left|N_{u}^{*}\right|=60 /(q+1)$. Further, if $q=19$, then $G_{0}=N^{*} \imath\langle\tau\rangle$, so we may take $H_{0}$ to be $N^{*}$, but $N^{*}$ is not regular on $V_{2}(p)^{*}$, a contradiction. Thus $q \in\{11,29,59\}$. Using Magma, we determine that $N^{*}$ has a subgroup $R$ that is regular on $V_{2}(p)^{*}$ and isomorphic to a group in Table 8. Moreover, $N^{*}=N_{\mathrm{GL}_{2}(p)}(R)$ and $R \times R \unlhd G_{0}$. Thus we may take $H_{0}$ to be $R$, in which case $\alpha^{2} t^{-1}=h \in H_{0}$ and $T_{u}$ is given by Table 8 , so (i)(b) holds.

Still assuming that $G_{0}$ lies in (I4), suppose that $q=9$. If $|L|=3$, then $L=\langle(u, u)\rangle_{\mathbb{F}_{3}}$ and $G_{0} \leqslant \Gamma L_{2}(9) \imath\langle\tau\rangle=\mathcal{G}\left(\mathrm{GL}_{2}(9),\langle u\rangle_{\mathbb{F}_{3}}, 1\right)$, so (i)(a) holds. Otherwise, $|L| \in\{9,81\}$ since $|L|$ divides 81 and $|B|$ divides 6400. By a computation in MAGMA and Examples 13.12 and 13.15, if $G_{0} \leqslant \mathcal{G}\left(\mathrm{GL}_{2}(9),\langle u\rangle_{\mathbb{F}_{9}}, 1\right)=\mathrm{GL}_{2}(9) \imath\langle\tau\rangle\langle(\sigma, \sigma)\rangle$, then (i)(a) holds, and if not, then (i)(d) holds.

Suppose that $G_{0}$ belongs to (I8). Then $\left(D_{8} \circ Q_{8}\right) \times\left(D_{8} \circ Q_{8}\right) \unlhd G_{0} \leqslant N^{*} \imath\langle\tau\rangle$ where $N^{*}:=$ $N_{\mathrm{GL}_{m}(q)}\left(D_{8} \circ Q_{8}\right), m=4$ and $q=p=3$. Now $-1 \in G_{0}$, so $L$ is an $\mathbb{F}_{3}$-subspace of $V$ by Lemma 2.16. In particular, $M$ is a subspace of $V_{4}(3)$ and $|L|$ divides $3^{4}$. If $|L|=3$, then $L=\langle(u, u)\rangle_{\mathbb{F}_{3}}$ and $G_{0} \leqslant \mathrm{GL}_{4}(3) \imath \tau=\mathcal{G}\left(\mathrm{GL}_{4}(3),\langle u\rangle_{\mathbb{F}_{3}}, 1\right)$, so (i) (a) holds. Otherwise, $|L|>3$. By Example 13.16, $\mathrm{GL}_{4}(3)$ has a subgroup $H_{0}:=\left(D_{8} \circ Q_{8}\right) . C_{5}$ that is transitive on $V_{4}(3)^{*}$. Further, $N:=N_{\mathrm{GL}_{4}(3)}\left(H_{0}\right)=\left(D_{8} \circ Q_{8}\right) . \mathrm{AGL}_{1}(5)=H_{0}\langle s\rangle$, where $N_{u}=\langle s\rangle \simeq C_{8}$ and $H_{0, u}=\left\langle s^{4}\right\rangle$. By a computation in MAGma, there exists $g \in N^{*} \times N^{*}$ such that $H_{0} \times H_{0} \unlhd G_{0}^{g}$. (Note that $H_{0}$ is not normal in $N^{*}$ since $N<\left(D_{8} \circ Q_{8}\right) \cdot S_{5}=N^{*}$ by Remark 2.20.) Thus we may assume that $H_{0} \times H_{0} \unlhd G_{0}=G_{0, V_{1}}\langle(t, 1) \tau\rangle$, in which case $M=C_{V_{4}(3)}\left(H_{0, u}\right) \simeq V_{2}(3)$ and (i)(e) holds. (The additional statements can be verified using MAGMA and Example 13.16.)

For Proposition 13.20(ii) and cases (a), (c), (d) and (e) of Proposition 13.20(i), the number of examples for a particular group $G$ can be determined directly from the conditions on $\alpha$, as described in Examples 13.17, 13.12, 13.14, 13.15 and 13.16, respectively. This can also be done for Proposition 13.20(i)(b) when $H_{0} \times H_{0} \unlhd G_{0}$ and $\alpha^{2} t^{-1} \in H_{0}$, as follows. Observe that if $T_{u}$ is abelian and $G_{0} \leqslant \mathcal{G}\left(H_{0}, V_{2}(p), \alpha_{1}\right) \cap \mathcal{G}\left(H_{0}, V_{2}(p), \alpha_{2}\right)$ for some $\alpha_{1}, \alpha_{2} \in T_{u}$, then $\alpha_{1}^{2}=r_{1} t$ and $\alpha_{2}^{2}=r_{2} t$ for some $r_{1}, r_{2} \in H_{0}$, so $\alpha_{1}^{2}=\alpha_{2}^{2}$ since $H_{0, u}=1$, in which case $\mathcal{G}\left(H_{0}, V_{2}(p), \alpha_{1}\right)=\mathcal{G}\left(H_{0}, V_{2}(p), \alpha_{2}\right)$ by Lemma 13.11 (cf. Example 13.13). In particular, if $T_{u}$ is cyclic of odd order, then $G_{0}$ admits a unique example, and if $T_{u}=C_{2}$ or $C_{4}$, then $G_{0}$ admits exactly two examples. Otherwise, $T_{u}=S_{3}$ and the groups $\mathcal{G}\left(H_{0}, V_{2}(3), \alpha\right)$ are pairwise distinct for $\alpha \in T_{u}$. In particular, both $\mathcal{G}\left(H_{0}, V_{2}(3), 1\right)$ and its subgroup $H_{0}\langle\langle\tau\rangle\{(s, s): s \in$ $\left.A_{3}\right\}$ admit exactly one example, while $H_{0}\left\langle\langle\tau\rangle\left\{\left(s, s^{-1}\right): s \in A_{3}\right\}=\bigcap_{\alpha \in S_{3} \backslash A_{3}} \mathcal{G}\left(H_{0}, V_{2}(3), \alpha\right)\right.$ admits exactly three examples. Up to conjugacy, these are all of the rank 3 groups. (Note that $H_{0} \prec\langle\tau\rangle=\bigcap_{\alpha \in\left(S_{3} \backslash A_{3}\right) \cup\{1\}} \mathcal{G}\left(H_{0}, V_{2}(3), \alpha\right)$ admits exactly four examples, but $H_{0}=Q_{8} \leqslant \Gamma L_{1}(9)$, so this group is not considered in Proposition 13.20.)

Proof of Proposition 13.3. This follows from Proposition 13.20, Lemma 13.11(iii), and the information given in Examples 13.12-13.17.

\section{The extraspecial Class (E)}

We begin by describing the exceptional partial linear spaces that arise for class (E). Recall the definition of $\zeta_{q}$ from $\S 2.3$.

Example 14.1. Let $E:=D_{8} \circ Q_{8}$, and let $G:=V: N_{\mathrm{GL}_{4}(5)}(E)$ where $V:=V_{4}(5)$. Now $G$ has rank 3 with subdegrees 240 and 384 . By a computation in MAGMA, $G_{0}=\left(\mathrm{SL}_{2}(5) \circ E \circ\left\langle\zeta_{5}\right\rangle\right) \cdot 2$, and the proper subgroups of $G_{0}$ with two orbits on $V^{*}$ are $\mathrm{SL}_{2}(5) \circ E$ and $\mathrm{SL}_{2}(5) \circ E \circ\left\langle\zeta_{5}\right\rangle$. The exceptional Walker plane of order 25 is an affine plane with group of automorphisms $G$, and $G$ has $\ell_{\infty}$-orbit lengths $(10,16)$ (see $[47,54]$ and [3, Corollary 8]). Now $G$ has two orbits $\mathcal{L}_{1}$ and $\mathcal{L}_{2}$ on the lines of this affine plane, so $\mathcal{S}_{1}:=\left(V, \mathcal{L}_{1}\right)$ and $\mathcal{S}_{2}:=\left(V, \mathcal{L}_{2}\right)$ are $G$-affine proper partial 
linear spaces by Lemma 2.11. Using Magma, we determine that $\operatorname{Aut}\left(\mathcal{S}_{1}\right)=\operatorname{Aut}\left(\mathcal{S}_{2}\right)=G$. Further, the lines of $\mathcal{S}_{1}$ and $\mathcal{S}_{2}$ are affine subspaces of $V$.

Example 14.2. Let $E:=D_{8} \circ Q_{8}$, and let $G:=V: N_{\mathrm{GL}_{4}(7)}(E)$ where $V:=V_{4}(7)$. Now $G$ has rank 3 with subdegrees 480 and 1920 . By a computation in MAGma, $G_{0}=2 \cdot S_{5}^{+} \circ E \circ\left\langle\zeta_{7}\right\rangle$, and the proper subgroups of $G_{0}$ with two orbits on $V^{*}$ are $2 \cdot S_{5}^{+} \circ E$ or a conjugate of $\left(E \circ\left\langle\zeta_{7}\right\rangle\right) \cdot \mathrm{AGL}_{1}(5)$. The Mason-Ostrom plane of order 49 is an affine plane with group of automorphisms $G$, and $G$ has $\ell_{\infty}$-orbit lengths $(10,40)$ (see [44] and [3, Corollary 8]). Now $G$ has two orbits $\mathcal{L}_{1}$ and $\mathcal{L}_{2}$ on the lines of this affine plane, so $\mathcal{S}_{1}:=\left(V, \mathcal{L}_{1}\right)$ and $\mathcal{S}_{2}:=\left(V, \mathcal{L}_{2}\right)$ are $G$-affine proper partial linear spaces by Lemma 2.11. Using Magma, we determine that $\operatorname{Aut}\left(\mathcal{S}_{1}\right)=\operatorname{Aut}\left(\mathcal{S}_{2}\right)=G$. Further, the lines of $\mathcal{S}_{1}$ and $\mathcal{S}_{2}$ are affine subspaces of $V$.

Example 14.3. Let $E:=Q_{8} \circ Q_{8} \circ Q_{8}$, and let $G:=V: N_{\mathrm{GL}_{8}(3)}(E)$ where $V:=V_{8}(3)$. By $[39$, p.485], $G_{0}=E \cdot \mathrm{SO}_{6}^{-}(2) \leqslant \mathrm{GSp}_{8}(3)$, and $G$ has rank 3 with subdegrees 1440 and 5120 . By a computation in MAGMA, the only proper subgroup of $G_{0}$ with two orbits on $V^{*}$ is $E \cdot \Omega_{6}^{-}(2)$. Now $G_{0}$ has an orbit $\Delta$ on the points of $\mathrm{PG}_{7}(3)$ with size 720 . By a computation in GAP using [2], there is a partition $\Sigma$ of $\Delta$ into hyperbolic lines - that is, projective lines arising from non-degenerate 2-subspaces of $V$ with respect to the symplectic form preserved by $\operatorname{Sp}_{8}(3)$ - such that $G_{0}$ acts transitively on the elements of $\Sigma$. Embed $\mathrm{PG}_{7}(3)$ as a hyperplane $\Pi$ in $\mathrm{PG}_{8}(3)$. View $V$ as the set of points in $\mathrm{AG}_{8}(3)$ (i.e., the set of points in $\mathrm{PG}_{8}(3)$ that are not in $\Pi$ ), and let $\mathcal{L}$ be the set of affine 2 -subspaces of $\mathrm{AG}_{8}(3)$ whose completions meet $\Pi$ in an element of $\Sigma$. Then $\mathcal{S}:=(V, \mathcal{L})$ is a $G$-affine proper partial linear space with line-size 9. By a computation in Magma, $\operatorname{Aut}(\mathcal{S})=G$.

Proposition 14.4. Let $G$ be an affine permutation group of rank 3 on $V:=V_{d}(p)$ where $p$ is prime, $E \unlhd G_{0}, E$ is irreducible on $V$ and $\left(E, p^{d}, G\right)$ is given by Table 2. Then $\mathcal{S}$ is a $G$-affine proper partial linear space if and only if one of the following holds.

(i) $\mathcal{S}=\left(V,\left\{\langle u\rangle_{\mathbb{F}_{p}}+v: u \in X, v \in V\right\}\right)$ where $X$ is an orbit of $G_{0}$ on $V^{*}$ and $p \neq 2$.

(ii) $\left(E, p^{d}\right)=\left(3^{1+2}, 2^{6}\right)$ and $\mathcal{S}=\left(V,\left\{\langle u\rangle_{\mathbb{F}_{4}}+v: u \in X, v \in V\right\}\right)$ where $V=V_{3}(4)$ and $X$ is an orbit of $G_{0}$ on $V^{*}$. Here $\mathcal{S}$ has an automorphism group in class (R1).

(iii) $\left(E, p^{d}\right)=\left(D_{8} \circ Q_{8}, 5^{4}\right)$ and $\mathcal{S}$ is described in Example 14.1.

(iv) $\left(E, p^{d}\right)=\left(D_{8} \circ Q_{8}, 7^{4}\right)$ and $\mathcal{S}$ is described in Example 14.2.

(v) $\left(E, p^{d}\right)=\left(Q_{8} \circ Q_{8} \circ Q_{8}, 3^{8}\right)$ and $\mathcal{S}$ is described in Example 14.3.

Proof. Note that by Corollary 3.5, $\langle x\rangle_{\mathbb{F}_{p}}^{*} \subseteq x^{G_{0}}$ for all $x \in V^{*}$. Further, if $\left(E, p^{d}\right)=\left(3^{1+2}, 2^{6}\right)$, then $G_{0} \leqslant \Gamma \mathrm{U}_{3}(2)$ and $V=V_{3}(4)$, so $\langle x\rangle_{\mathbb{F}_{4}}^{*} \subseteq x^{G_{0}}$ for all $x \in V^{*}$. In particular, if (i) or (ii) holds, then $\mathcal{S}$ is a $G$-affine proper partial linear space, and when (ii) holds, $V: \Gamma U_{3}(2)$ is an automorphism group of $\mathcal{S}$ in class (R1). Moreover, if one of (iii)-(v) holds, then we have already seen in Examples 14.1-14.3 that $\mathcal{S}$ is a $G$-affine proper partial linear space.

Suppose that $\mathcal{S}$ is a $G$-affine proper partial linear space. Let $L \in \mathcal{L}_{0}$, let $B:=L^{*}$, and let $x \in B$. Since $E$ is irreducible, $G$ is primitive by Lemma 2.1, so $B$ is a non-trivial block of $G_{0}$ on $x^{G_{0}}$ by Lemma 2.6. If $p$ is odd, then since $E$ is irreducible, its central involution is

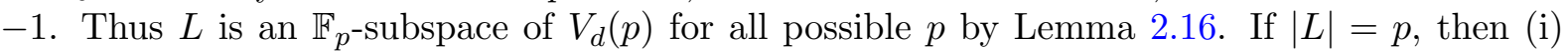
holds, so we assume that $|L| \geqslant p^{2}$. In particular, $d>2$. If $\left(E, p^{d}\right)=\left(D_{8} \circ Q_{8} \circ\left\langle\zeta_{5}\right\rangle, 5^{4}\right)$ and $G_{0} \nless N_{\mathrm{GL}_{4}(5)}\left(D_{8} \circ Q_{8}\right)$, then there are no examples by a computation in Magma. Otherwise, $\left(E, p^{d}\right)$ is one of $\left(3^{1+2}, 2^{6}\right),\left(D_{8} \circ Q_{8}, 5^{4}\right),\left(D_{8} \circ Q_{8}, 7^{4}\right)$ or $\left(Q_{8} \circ Q_{8} \circ Q_{8}, 3^{8}\right)$, in which case one of (ii)-(v) holds by a computation in MAGMA.

\section{The almost simple Class (AS)}

We begin by describing the exceptional partial linear spaces that arise for class (AS). Note that when computing with MAGMA in these examples, we often use [56] to construct the representation of the appropriate quasisimple group. Recall the definition of $\zeta_{q}$ and $\sigma_{q}$ from $\S 2.3$. 
Example 15.1. Let $S:=2 \cdot A_{5} \simeq \mathrm{SL}_{2}(5)$. By [23, Lemma 11.2], the normaliser $N$ of $S$ in $\Gamma L_{2}(9)$ is $S\langle\zeta, \sigma\rangle$ where $\zeta:=\zeta_{9}$ and $\sigma:=\sigma_{9}$, and $N$ is transitive on $V^{*}$ where $V:=V_{2}(9)$. Moreover, by [25, Theorem 5.3], the subgroups of $N$ containing $S$ with two orbits on $V^{*}$ are $S, S\left\langle\zeta^{2}\right\rangle, S\langle\sigma\rangle$, $S\left\langle\zeta^{2} \sigma\right\rangle$ and $S\left\langle\zeta^{2}, \sigma\right\rangle$; these all have orbit sizes 40 and 40 on $V^{*}$. Note that $S\langle\sigma\rangle^{\zeta}=S\left\langle\zeta^{2} \sigma\right\rangle \simeq$ $2 \cdot S_{5}^{+}$. The nearfield plane of order 9 is an affine plane whose full automorphism group is the 2-transitive group $V:\left(D_{8} \circ Q_{8} \circ S\langle\zeta \sigma\rangle\right)$ (see $\S 2.9$ and Remark 2.20), and $V: S$ has $\ell_{\infty}$-orbit lengths $(5,5)$ (see $\left[3\right.$, Corollary 8]). Now $V: S$ has two orbits $\mathcal{L}_{1}$ and $\mathcal{L}_{2}$ on the lines of the nearfield plane, so $\mathcal{S}_{1}:=\left(V, \mathcal{L}_{1}\right)$ and $\mathcal{S}_{2}:=\left(V, \mathcal{L}_{2}\right)$ are $(V: S)$-affine proper partial linear spaces by Lemma 2.11 . Using Magma, we determine that, without loss of generality, $\operatorname{Aut}\left(\mathcal{S}_{1}\right)=V: S\langle\sigma\rangle$ and $\operatorname{Aut}\left(\mathcal{S}_{2}\right)=$ $V: S\left\langle\zeta^{2} \sigma\right\rangle$. Thus $\mathcal{S}_{1}$ is a $\left(V: G_{0}\right)$-affine proper partial linear space for $G_{0} \in\{S, S\langle\sigma\rangle\}$, and $\mathcal{S}_{2}$ is a $\left(V: G_{0}\right)$-affine proper partial linear space for $G_{0} \in\left\{S, S\left\langle\zeta^{2} \sigma\right\rangle\right\}$. Note that $\mathcal{S}_{1} \simeq \mathcal{S}_{2}$ since $\zeta \sigma$ maps $\mathcal{L}_{1}$ to $\mathcal{L}_{2}$. Note also that the lines of $\mathcal{S}_{1}$ are affine $\mathbb{F}_{3}$-subspaces of $V$.

Example 15.2. Let $S:=2 \cdot A_{5} \simeq \mathrm{SL}_{2}(5)$ and $q:=49$. By a computation in Magma, the normaliser $N$ of $S$ in $\Gamma L_{2}(q)$ is $S\langle\zeta, \sigma\rangle$ where $\zeta:=\zeta_{q}$ and $\sigma:=\sigma_{q}$, and $N$ has orbits $X$ and $Y$ on $V^{*}$ with sizes 960 and 1440 , respectively, where $V:=V_{2}(q)$. Moreover, the proper subgroups of $N$ with two orbits on $V^{*}$ are $S \circ\langle\zeta\rangle$ and $S\left\langle\zeta \sigma, \zeta^{2}\right\rangle=2 \cdot S_{5}^{-} \circ\left\langle\zeta^{2}\right\rangle$. Let $G:=V: S\left\langle\zeta \sigma, \zeta^{2}\right\rangle$. The Korchmáros plane of order 49 is an affine plane with group of automorphisms $G$, and $G$ has $\ell_{\infty}$-orbit lengths $(20,30)[38]$. Now $G$ has two orbits on the lines of this affine plane; let $\mathcal{L}$ be the orbit for which $\left|\mathcal{L}_{0}\right|=20$. By Lemma $2.11, \mathcal{S}:=(V, \mathcal{L})$ is a $G$-affine proper partial linear space with $\mathcal{S}(0)=X$, and using Magma, we determine that $\operatorname{Aut}(\mathcal{S})=G$ and $\mathcal{L}$ consists of affine $\mathbb{F}_{7^{-}}$ subspaces of $V$. We do not consider the other partial linear space arising from the Korchmáros plane here, for it is $\mathbb{F}_{q^{-}}$-dependent with line set $\left\{\langle u\rangle_{\mathbb{F}_{q}}+v: u \in Y, v \in V\right\}$. Further, $\mathcal{S}$ is not

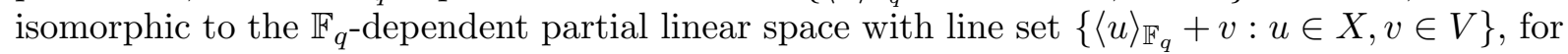
the automorphism group of this latter partial linear space contains $V: S\langle\zeta, \sigma\rangle$.

Example 15.3. Let $S:=2 \cdot A_{6}$. By a computation in MAGMA, the normaliser $N$ of $S$ in $\mathrm{GL}_{4}(5)$ is $\left(S \circ\left\langle\zeta_{5}\right\rangle\right) \cdot 2$, and $N$ has orbit sizes 144 and 480 on $V^{*}$ where $V:=V_{4}(5)$. Moreover, $N$ has no proper subgroups with two orbits on $V^{*}$. Let $G:=V: N$. The exceptional Walker plane of order 25, or Hering plane, is an affine plane with group of automorphisms $G$, and $G$ has $\ell_{\infty}$-orbit lengths $(6,20)$ (see [54] and [3, Corollary 8]). Now $G$ has two orbits $\mathcal{L}_{1}$ and $\mathcal{L}_{2}$ on the lines of this affine plane, so $\mathcal{S}_{1}:=\left(V, \mathcal{L}_{1}\right)$ and $\mathcal{S}_{2}:=\left(V, \mathcal{L}_{2}\right)$ are $G$-affine proper partial linear spaces by Lemma 2.11. Using Magma, we determine that $\operatorname{Aut}\left(\mathcal{S}_{1}\right)=\operatorname{Aut}\left(\mathcal{S}_{2}\right)=G$. Further, the lines of $\mathcal{S}_{1}$ and $\mathcal{S}_{2}$ are affine subspaces of $V$.

Example 15.4. Let $S:=A_{9}$. By [39, p.509], the group $G:=V: S$ has rank 3 , where $V:=V_{8}(2)$ and $S \leqslant \Omega_{8}^{+}(2)$. The orbits of $S$ on $V^{*}$ are the sets of non-singular and singular vectors with respect to the quadratic form preserved by $\Omega_{8}^{+}(2)$, with sizes 120 and 135 , respectively. By a computation in Magma, $S$ has no proper subgroups with two orbits on $V^{*}$. The following definition is made in $[10, \S 2.3 .3]$. Let $\Delta$ be the set of points of $\mathrm{PG}_{7}(2)$ that are singular with respect to the quadratic form preserved by $\Omega_{8}^{+}(2)$. By [21], there exists a spread $\Sigma$ of $\Delta$ - that is, a partition of the points of $\Delta$ into maximal totally singular subspaces - such that $S$ acts 2-transitively on the elements of $\Sigma$. Embed $\mathrm{PG}_{7}(2)$ as a hyperplane $\Pi$ in $\mathrm{PG}_{8}(2)$. View $V$ as the set of points in $\mathrm{AG}_{8}(2)$ (i.e. the set of points in $\mathrm{PG}_{8}(2)$ that are not in $\Pi$ ), and let $\mathcal{L}$ be the set of affine 4 -subspaces of $\mathrm{AG}_{8}(2)$ whose completions meet $\Pi$ in an element of $\Sigma$. Then $\mathcal{S}:=(V, \mathcal{L})$ is a $G$-affine proper partial linear space with line-size 16. By $[10, \S 2.3 .3], \operatorname{Aut}(\mathcal{S})=G$.

Example 15.5. Let $S:=M_{11}$. Let $V$ be the irreducible $\mathbb{F}_{3} S$-module of dimension 5 such that $S$ has orbit sizes 110 and 132 on $V^{*}$. Let $G:=V: S$. Let $x$ be an element of the orbit of $S$ of size 132 on $V^{*}$. Then $S_{x} \simeq A_{5}$, and there are exactly two maximal subgroups $H$ of $S$ for which $S_{x} \leqslant H \simeq \mathrm{PSL}_{2}(11)$. Let $B:=x^{H}$. Now $B$ is a block of $S$. Let $L:=B \cup\{0\}$ and $\mathcal{L}:=L^{G}$. By a computation in MAGMA, $S_{L}$ is transitive on $L$, so by Lemma 2.7, $\mathcal{S}:=(V, \mathcal{L})$ is a $G$-affine proper partial linear space with line-size 12 and point-size 12. By a computation in MAgma, $\operatorname{Aut}(\mathcal{S})=G$. Note that if we instead choose $H$ to be the other maximal subgroup of $S$ for which 
$S_{x} \leqslant H \simeq \mathrm{PSL}_{2}(11)$, then we obtain another $G$-affine proper partial linear space, but we will see in Proposition 15.8 that these partial linear spaces are isomorphic. Note also that $S$ has no proper subgroups with two orbits on $V^{*}$.

Example 15.6. Let $S:=2 \cdot G_{2}(4)$. By a computation in MAgma, the normaliser $N$ of $S$ in $\mathrm{GL}_{12}(3)$ is $S .2^{-}$, and $N$ has orbit sizes 65520 and 465920 on $V^{*}$ where $V:=V_{12}(3)$. Moreover, the only proper subgroup of $N$ with two orbits on $V^{*}$ is $S$. Recall from Table 3 that $S \leqslant \operatorname{Sp}_{12}(3)$, so $N \leqslant \operatorname{GSp}_{12}(3)$ by [37, Corollary $\left.2.10 .4(\mathrm{i})\right]$. Let $G:=V: N$. Now $G_{0}$ has an orbit $\Delta$ on the points of $\mathrm{PG}_{11}(3)$ with size 32760 . By a computation in GAP using [2], there is a partition $\Sigma$ of $\Delta$ into hyperbolic lines - that is, projective lines arising from non-degenerate 2-subspaces of $V$ with respect to the symplectic form preserved by $\operatorname{Sp}_{12}(3)$ - such that $G_{0}$ acts transitively on the elements of $\Sigma$. Embed $\mathrm{PG}_{11}(3)$ as a hyperplane $\Pi$ in $\mathrm{PG}_{12}(3)$. View $V$ as the set of points in $\mathrm{AG}_{12}(3)$, and let $\mathcal{L}$ be the set of affine 2-subspaces of $\mathrm{AG}_{12}(3)$ whose completions meet $\Pi$ in an element of $\Sigma$. Then $\mathcal{S}:=(V, \mathcal{L})$ is a $G$-affine proper partial linear space with line-size 9 . We claim that $\operatorname{Aut}(\mathcal{S})=G$. By Theorem 1.8, $\operatorname{Aut}(\mathcal{S})$ is an affine permutation group on $V$. A consideration of the subdegrees in Tables $2-4$ shows that $\operatorname{Aut}(\mathcal{S})$ belongs to class $($ AS $)$ with $S=2 \cdot G_{2}(4)$ or $2 \cdot$ Suz. It then follows from Proposition 15.8 below that $\operatorname{Aut}(\mathcal{S})=G$.

Example 15.7. Let $S:=2 \cdot J_{2}$. By a computation in MAGMA, the normaliser $N$ of $S$ in $\mathrm{GL}_{6}(5)$ is $\left(S \circ\left\langle\zeta_{5}\right\rangle\right) \cdot 2$, and $N$ has orbit sizes 7560 and 8064 on $V^{*}$ where $V:=V_{6}(5)$. Moreover, the proper subgroups of $N$ with two orbits on $V^{*}$ are $S$ and $S \circ\left\langle\zeta_{5}\right\rangle$. Recall from Table 3 that $S \leqslant \operatorname{Sp}_{6}(5)$, so $N \leqslant \operatorname{GSp}_{6}(5)$ by [37, Corollary 2.10.4(i)]. Let $G:=V: N$. Now $G_{0}$ has an orbit $\Delta$ on the points of $\mathrm{PG}_{5}(5)$ with size 1890 . By the proof of [39, Lemma 5.1], there is a partition $\Sigma$ of $\Delta$ into hyperbolic lines - that is, projective lines arising from non-degenerate 2 -subspaces of $V$ with respect to the symplectic form preserved by $\operatorname{Sp}_{6}(5)$ - such that $G_{0}$ acts transitively on the elements of $\Sigma$. (This partition arises from the 315 quaternionic reflections described in [14, p.42]: these reflections form a conjugacy class $C$ of involutions of $S$, and for each $r \in C$, there is a unique non-degenerate 2-subspace of $V$ on which $r$ acts as -1 , and $C_{S}(r)$ acts as $\mathrm{SL}_{2}(5)$ on this 2-subspace.) Embed $\mathrm{PG}_{5}(5)$ as a hyperplane $\Pi$ in $\mathrm{PG}_{6}(5)$. View $V$ as the set of points in $\mathrm{AG}_{6}(5)$, and let $\mathcal{L}$ be the set of affine 2 -subspaces of $\mathrm{AG}_{6}(5)$ whose completions meet $\Pi$ in an element of $\Sigma$. Then $\mathcal{S}:=(V, \mathcal{L})$ is a $G$-affine proper partial linear space with line-size 25 . By a computation in Magma, $\operatorname{Aut}(\mathcal{S})=G$.

Proposition 15.8. Let $G$ be an affine permutation group of rank 3 on $V:=V_{n}(q)$ where $q^{n}=p^{d}$ for $p$ prime, $S \unlhd G_{0}, S$ is irreducible on $V_{d}(p)$ and $\left(S, p^{d}, G, q\right)$ is given by Table 3 . Let $\zeta:=\zeta_{q}$. Then $\mathcal{S}$ is a $G$-affine proper partial linear space if and only if one of the following holds.

(i) $\mathcal{S}=\left(V,\left\{\langle u\rangle_{\mathbb{F}_{p}}+v: u \in X, v \in V\right\}\right)$ where $X$ is an orbit of $G_{0}$ on $V^{*}$ and $p \neq 2$.

(ii) $\left(S, p^{d}\right)$ is one of $\left(2 \cdot A_{5}, 7^{4}\right),\left(3 \cdot A_{6}, 2^{6}\right)$ or $\left(J_{2}, 2^{12}\right)$ and $\mathcal{S}=\left(V,\left\{\langle u\rangle_{\mathbb{F}_{q}}+v: u \in X, v \in V\right\}\right)$ where $X$ is an orbit of $G_{0}$ on $V^{*}$. Here $q=p^{2}$.

(iii) $\left(S, p^{d}\right)=\left(2 \cdot A_{5}, 3^{4}\right)$ and $G_{0}$ and $\mathcal{S}$ or $\mathcal{S}^{\zeta^{2}}$ are described in Example 15.1.

(iv) $\left(S, p^{d}\right)=\left(2 \cdot A_{5}, 7^{4}\right)$ and $G_{0}$ and $\mathcal{S}$ or $\mathcal{S}^{\zeta}$ are described in Example 15.2.

(v) $\left(S, p^{d}\right)=\left(2 \cdot A_{6}, 5^{4}\right)$ and $\mathcal{S}$ is described in Example 15.3 .

(vi) $\left(S, p^{d}\right)=\left(A_{9}, 2^{8}\right)$ and $\mathcal{S}$ is described in Example 15.4 .

(vii) $\left(S, p^{d}\right)=\left(M_{11}, 3^{5}\right)$ and $G_{0}$ and $\mathcal{S}$ or $\mathcal{S}^{\zeta}$ are described in Example 15.5.

(viii) $\left(S, p^{d}\right)=\left(2 \cdot G_{2}(4), 3^{12}\right)$ and $\mathcal{S}$ is described in Example 15.6.

(ix) $\left(S, p^{d}\right)=\left(2 \cdot J_{2}, 5^{6}\right)$ and $\mathcal{S}$ is described in Example 15.7.

Proof. By assumption, $S$ is irreducible on $V_{d}(p)$, so $G$ is primitive by Lemma 2.1. Let $\sigma:=\sigma_{q}$. By Corollaries 3.5 and 3.7, $\langle x\rangle_{\mathbb{F}_{q}}^{*} \subseteq x^{G_{0}}$ for all $x \in V^{*}$, except when $S=2 \cdot A_{5}$ and $q=9$, in which case $\langle x\rangle_{\mathbb{F}_{3}}^{*} \subseteq x^{G_{0}}$ for all $x \in V^{*}$. In particular, if (i) or (ii) holds, then $\mathcal{S}$ is a $G$-affine proper partial linear space. Moreover, if one of (iii)-(ix) holds, then we have already seen in Examples 15.1-15.7 that $\mathcal{S}$ is a $G$-affine proper partial linear space. 
Suppose that $\mathcal{S}$ is a $G$-affine proper partial linear space. Let $L \in \mathcal{L}_{0}$, let $B:=L^{*}$, and let $x \in B$. By Lemma 2.6, $B$ is a non-trivial block of $G_{0}$ on $X:=x^{G_{0}}$. First suppose that $S \neq M_{11}$. Then $-1 \in G_{0}$ (including the case $p=2$ ), so $L$ is an $\mathbb{F}_{p}$-subspace of $V$ by Lemma 2.16. If $|L|=p$, then $p \neq 2$ and (i) holds. Thus we may assume that $|L|>p$, so $d>2$. Using Magma and [56], we verify that one of (ii)-(vi), (viii) or (ix) holds.

Thus $\left(S, p^{d}\right)=\left(M_{11}, 3^{5}\right)$ and $q=3$. If $B=\{x,-x\}$, then (i) holds, so we assume otherwise. By a computation in Magma, either $|X|=220, G_{0} \in\left\{S, S \times \mathbb{F}_{3}^{*}\right\}$ and $|B|=4$ where $B$ is the same block for $S$ and $S \times \mathbb{F}_{3}^{*}$; or $|X|=132, G_{0}=S$ and $B$ is one of two blocks with size 11. In the latter case, (vii) holds. In the former case, $|L|$ does not divide $3^{5}$, but $-1 \in\left(S \times \mathbb{F}_{3}^{*}\right) \backslash S$, so we obtain a contradiction using Lemmas 2.6, 2.7 and 2.16.

Note that the partial linear space in Proposition 15.8(ii) with $S=3 \cdot A_{6}$ and $|X|=18$ is the unique generalised quadrangle of order $(3,5)$; see [11] for more details.

\section{Proof of Theorem 1.4}

Let $\mathcal{S}$ be a finite proper partial linear space, and let $G \leqslant \operatorname{Aut}(\mathcal{S})$ where $G$ is an affine primitive permutation group of rank 3 with socle $V:=V_{d}(p)$ where $d \geqslant 1$ and $p$ is prime. We may assume that $\mathcal{S}$ has point set $V$. Let $k$ be the line-size of $\mathcal{S}$, and let $\ell$ be the point-size of $\mathcal{S}$. By Lemma $2.8,|\mathcal{S}(0)|=\ell(k-1)$.

Suppose that $\mathcal{S}$ is described in one of Examples 6.2, 13.6, 13.8, 13.13-13.17, 14.1-14.3 or 15.115.7. By the information in these examples, in order to prove that (iii) holds, it remains to justify the comments in the column "Notes" for Examples 6.2, 13.8 (with line-size 27), 14.3 and 15.4-15.7, where we claim that $\mathcal{S}$ cannot be obtained from any $2-\left(p^{d}, k, 1\right)$ design admitting a rank 3 group (by the method of Lemma 2.11), as well as Examples 13.6, 13.8 (with linesize 9$)$ and 13.14-13.16, where we claim that $\mathcal{S}$ can be obtained from a rank 3 affine $2-\left(p^{d}, k, 1\right)$ design. By joining the lines of a partial linear space of line-size 9 from Example 13.8 with the lines of a partial linear space from Example 13.14, both of which admit $3^{12}$ : $\left(\mathrm{SL}_{2}(13)\right.$ ? $\left.S_{2}\right)$ as an automorphism group, we obtain a rank 3 affine $2-\left(3^{12}, 9,1\right)$ design by Lemma 2.11. Similarly, we obtain a rank 3 affine $2-\left(3^{8}, 9,1\right)$ design by joining the lines of a partial linear space from Example 13.6 with the lines of a partial linear space from Example 13.15 (respectively, 13.16), both of which admit $3^{8}:\left(2 \cdot S_{5}^{-} \prec S_{2}\right)$ (respectively, $\left.3^{8}:\left(\left(\left(D_{8} \circ Q_{8}\right) \cdot D_{10}\right) \curlywedge S_{2}\right)\right)$ as an automorphism group.

Now suppose that $\mathcal{S}$ is described in Examples 6.2, 13.8 (with line-size 27), 14.3 or 15.4-15.7, and suppose that there exists a permutation group $H$ on $V$ that is transitive of rank 3 and an automorphism group of a $2-\left(p^{d}, k, 1\right)$ design $\mathcal{D}$ with point set $V$ such that $H$ has orbits $\mathcal{L}_{1}$ and $\mathcal{L}_{2}$ on the lines of $\mathcal{D}$, where $\mathcal{L}_{1}$ is the line set of $\mathcal{S}$. Now $H \leqslant \operatorname{Aut}(\mathcal{S})$, and $\operatorname{Aut}(\mathcal{S})$ is an affine primitive permutation group with socle $V$. We claim that $V \leqslant H$. Note that $|H|=|V|\left|H_{0}\right|$ and $H_{0}$ has two orbits on $V^{*}$. In particular, by the information given in the examples, either $H_{0}=\operatorname{Aut}(\mathcal{S})_{0}$, or $H_{0}$ is one of the following: $\left(Q_{8} \circ Q_{8} \circ Q_{8}\right) \cdot \Omega_{6}^{-}(2)$ when $p^{d}=3^{8} ; 2 \cdot G_{2}(4)$ when $p^{d}=3^{12}$; or one of $2 \cdot J_{2}$ or $2 \cdot J_{2} \circ 4$ when $p^{d}=5^{6}$. In particular, $\left[\operatorname{Aut}(\mathcal{S})_{0}: H_{0}\right]=2^{i}$ for some $i$, and $i=0$ when $p=2$. However $[V: V \cap H]=[H V: H]$, and this divides $[\operatorname{Aut}(\mathcal{S}): H]=\left[\operatorname{Aut}(\mathcal{S})_{0}: H_{0}\right]$, so the claim holds. Now $\left(V, \mathcal{L}_{2}\right)$ is an $H$-affine proper partial linear space with line-size $k$ by Lemma 2.11, but no such partial linear space exists by Propositions 6.3, 13.3, 14.4 and 15.8 since $\mathrm{SL}_{2}(13)$ is not a subgroup of $\Gamma L_{2}(27)$. Thus (iii) does indeed hold.

For the remainder of this proof, we say that a triple $(H, a, r)$ is good if it satisfies Hypothesis 3.6 and $H$ has socle $V$, in which case $H$ is primitive on $V$ and $r^{a}=p^{d}$, and by Corollary 3.7, $H_{0} \leqslant \Gamma \mathrm{L}_{a}(r)$ and $H_{0}$ has two orbits on the points of $\mathrm{PG}_{a-1}(r)$.

By Theorem 3.1, $G_{0}$ belongs to one of the classes (R0)-(R5), (T1)-(T3), (S0)-(S2), (I0)-(I8), (E) or (AS). If $G_{0}$ belongs to the class (R0), then (iv)(a) holds. If $G_{0}$ belongs to class (E), then by Proposition 14.4, either $\mathcal{S}$ is described in Examples 14.1-14.3 and (iii) holds, or $\mathcal{S}$ is described 
in Example 1.1 with respect to the good triple $(G, d, p)$ when $p$ is odd or $(G, 3,4)$ when $p=2$, in which case (i) holds. Similarly, if $G_{0}$ belongs to class (AS), then by Proposition 15.8, either $\mathcal{S}$ is described in Examples 15.1-15.7 and (iii) holds, or $\mathcal{S}$ is described in Example 1.1 with respect to the good triple $(G, d, p)$ when $p \neq 2$ or the good triple $\left(G, d / 2, p^{2}\right)$ when $S \unlhd G_{0}$ and $\left(S, p^{d}\right)$ is one of $\left(2 \cdot A_{5}, 7^{4}\right),\left(3 \cdot A_{6}, 2^{6}\right)$ or $\left(J_{2}, 2^{12}\right)$, in which case (i) holds.

Suppose that $G_{0}$ belongs to one of the classes (R1)-(R5), (T1)-(T3) or (S0)-(S2). Now $V=$ $V_{c}(s)$ and $G_{0} \leqslant \Gamma L_{c}(s)$ where $(c, s)$ is given by Table 6 of Corollary 3.5, and $\langle x\rangle_{\mathbb{F}_{s}}^{*} \subseteq x^{G_{0}}$ for all $x \in V^{*}$. If $\mathcal{S}$ is $\mathbb{F}_{s}$-dependent, then by Proposition $4.2, \mathcal{S}$ has line set $\left\{\langle u\rangle_{\mathbb{F}_{r}}+v: u \in \mathcal{S}(0), v \in V\right\}$ for some subfield $\mathbb{F}_{r}$ of $\mathbb{F}_{s}$ with $r>2$, so $\mathcal{S}$ is described in Example 1.1 with respect to the good triple $\left(G, \log _{r}\left(p^{d}\right), r\right)$ (see the discussion before Proposition 4.2). Otherwise, $\mathcal{S}$ is $\mathbb{F}_{s}$-independent. If $G_{0}$ belongs to one of the classes (R1)-(R5), then by Propositions 5.1, 6.1, 6.3, 7.1, 8.1, 9.4 and $9.5, \mathcal{S}$ is described in Example 6.2, so (iii) holds. If $G_{0}$ belongs to one of the classes (T1)(T3), then by Proposition 10.1, $\mathcal{S}$ is described in Example 1.2, and (ii) holds. If $G_{0}$ belongs to $(\mathrm{S} 1)$ or $(\mathrm{S} 2)$, then by Propositions 11.1 and 11.2, either $\mathcal{S}$ is described in Example 1.2 and (ii) holds, or $s=9$ and $\mathcal{S}$ is isomorphic to a partial linear space from Example 1.1 that is defined with respect to the good triple $(G, c, s)$, so (i) holds. Lastly, suppose that $G_{0}$ belongs to (S0). If $|\mathcal{S}(0)|=q\left(q^{3}-1\right)\left(q^{2}-1\right)$, then (iv)(c) holds. Otherwise, by Proposition 12.1, $\mathcal{S}$ is described in Example 1.2, so (ii) holds.

We may therefore assume that $G_{0}$ belongs to one of the classes (I0)-(I8). Now $V=V_{n}(p) \oplus$ $V_{n}(p)$. Let $V_{1}:=\left\{(u, 0): u \in V_{n}(p)\right\}$ and $V_{2}:=\left\{(0, u): u \in V_{n}(p)\right\}$, and recall that $\mathcal{S}(0)=$ $V_{1}^{*} \cup V_{2}^{*}$ or $V_{n}(p)^{*} \times V_{n}(p)^{*}$. First suppose that $\mathcal{S}(0)=V_{1}^{*} \cup V_{2}^{*}$. By Proposition 13.1, one of the following holds: $\mathcal{S}$ is the $p^{n} \times p^{n}$ grid of Example 1.3, in which case (ii) holds; $\mathcal{S}$ is isomorphic to a partial linear space in Example 13.6 or 13.8, in which case (iii) holds; or $\mathcal{S}=\mathrm{AG}_{b}(r) \square \mathrm{AG}_{b}(r)$ where $r^{b}=p^{n}, b \geqslant 2$ and $r>2$, in which case $\mathcal{S}$ is described in Example 1.1 with respect to the good triple $\left(V:\left(\left(\Gamma \mathrm{L}_{b}(r) 2 S_{2}\right) \cap \Gamma \mathrm{L}_{2 b}(r)\right), 2 b, r\right)$ by Lemma 2.2, and (i) holds. Suppose instead that $\mathcal{S}(0)=V_{n}(p)^{*} \times V_{n}(p)^{*}$. If $G_{0}$ lies in class (I0), then (iv)(b) holds, so we assume otherwise. By Proposition 13.3, either $\mathcal{S}$ is isomorphic to a partial linear space described in Examples 13.1313.17, in which case (iii) holds, or $G_{0} \leqslant\left(\Gamma \mathrm{L}_{b}(r) \prec S_{2}\right) \cap \Gamma \mathrm{L}_{2 b}(r)$ and $\mathcal{S}$ is isomorphic to a partial linear space with line set $\left\{\langle u\rangle_{\mathbb{F}_{r}}+v: u \in V_{n}(p)^{*} \times V_{n}(p)^{*}, v \in V\right\}$ where $r^{b}=p^{n}, b \geqslant 2$ and $r>2$, in which case (i) holds since this partial linear space is described in Example 1.1 with respect to the good triple $(G, 2 b, r)$.

\section{Proof of Corollary 1.7}

First we require the following result. Recall the definition of $\zeta_{q}$ and $\sigma_{q}$ from $\S 2.3$.

Lemma 17.1. Let $G_{0} \leqslant \Gamma L_{1}\left(p^{d}\right)$ where $d \geqslant 1$ and $p$ is an odd prime. Suppose that $G_{0}$ has $t$ orbits on $V_{1}\left(p^{d}\right)^{*}$ where $t \leqslant 2$, and suppose that $\left|G_{0}\right|$ is even when $t=2$. Then $-1 \in G_{0}$.

Proof. Let $q:=p^{d}, \zeta:=\zeta_{q}$ and $\sigma:=\sigma_{q}$. Let $H:=G_{0} \cap\langle\zeta\rangle$. By [25, §3], $G_{0}=\left\langle\zeta^{n}, \zeta^{k} \sigma^{s}\right\rangle$ for some integers $n, k$ and $s$ such that $H=\left\langle\zeta^{n}\right\rangle,|H|=(q-1) / n,\left|G_{0}\right|=d(q-1) /$ sn and $s$ divides $d$. Since $-1 \in G_{0}$ if and only if $|H|$ is even, it suffices to prove that $(q-1) / n$ is even. Since $q$ is odd, we may assume that $n$ is even, and since $\left|G_{0}\right|$ is even and $s$ divides $d$, we may assume that $d$ is even. Write $d=2^{e} d^{\prime}$ and $n=2^{f} n^{\prime}$ where $d^{\prime}$ and $n^{\prime}$ are odd. If $t=2$, then since $n$ is even, the orbits of $G_{0}$ on $V^{*}$ have the same size by [25, Corollary 3.8], in which case $n / 2$ divides $d$ by [25, Theorem 3.10]. If $t=1$, then $n$ divides $d$ by [23, Proposition 15.3]. Thus, in either case, $f \leqslant e+1$, so it suffices to prove that $2^{e+2}$ divides $q-1$. Let $r:=p^{d^{\prime}}$. Since $e \geqslant 1$,

$$
q-1=r^{2^{e}}-1=(r-1)\left(r^{2^{0}}+1\right)\left(r^{2^{1}}+1\right) \cdots\left(r^{2^{e-1}}+1\right) .
$$

Since 4 divides $r-1$ or $r+1$, it follows that $2^{e+2}$ divides $q-1$, as desired.

Note that $\Gamma L_{1}(q)$ may contain odd-order subgroups with two orbits on $V_{1}(q)^{*}$ : if $q \equiv 3 \bmod 4$, then $G_{0}:=\left\{\lambda^{2}: \lambda \in \mathbb{F}_{q}^{*}\right\}$ has odd order, and the orbits of $G_{0}$ on $\mathbb{F}_{q}^{*}$ are $G_{0}$ and $-G_{0}$. 
Proof of Corollary 1.7. We may assume that $\mathcal{S}$ has point set $V$. Suppose that the lines of $\mathcal{S}$ are not affine subspaces of $V$. Now Theorem 1.4(iii) or (iv) holds. If Theorem 1.4(iii) holds, then by the descriptions of $\mathcal{S}$ given in Examples 6.2, 13.6, 13.8, 13.13-13.17, 14.1-14.3 and 15.1-15.7, either (i) or (ii) holds. Thus we may assume that Theorem 1.4(iv) holds. By Lemma 2.16, $p$ is odd and $G_{0}$ does not contain -1. In particular, Theorem 1.4(iv)(c) does not hold. If Theorem 1.4(iv)(a) holds, then $G_{0} \leqslant \Gamma L_{1}\left(p^{d}\right)$ and $G_{0}$ has two orbits on $V^{*}$, so by Lemma $17.1,\left|G_{0}\right|$ is odd. However, this contradicts the fact that the orbitals of $G$ are self-paired by Remark 2.5.

Hence Theorem 1.4(iv)(b) holds. Now $V=V_{n}(p) \oplus V_{n}(p)$ and $G_{0} \leqslant \Gamma L_{1}\left(p^{n}\right)$ 々 $S_{2}$ where $\mathcal{S}(0)=V_{n}(p)^{*} \times V_{n}(p)^{*}$. In order to show that (iii) holds, it remains to show that (2)-(7) hold and $n \geqslant 2$. Now (2) holds by Lemma 2.17, (3) holds by Lemma 4.4, (4) holds by Lemma 13.18, and (5) holds by Lemma 2.16. If $H \times K \leqslant G_{0}$ for some $H, K \leqslant \Gamma L_{1}\left(p^{n}\right)$ and if $H$ and $K$ are both transitive on $V_{n}(p)^{*}$, then $-1 \in H$ and $-1 \in K$ by Lemma 17.1, so $-1 \in G_{0}$, a contradiction. Thus (7) holds. Let $V_{1}:=\left\{(u, 0): u \in V_{n}(p)\right\}$ and $V_{2}:=\left\{(0, u): u \in V_{n}(p)\right\}$, and for $i \in\{1,2\}$, let $G_{0}^{i}$ be the image of the projection of $G_{0, V_{1}}=G_{0} \cap\left(\Gamma L_{1}\left(p^{n}\right) \times \Gamma L_{1}\left(p^{n}\right)\right)$ onto the $i$-th factor of $\Gamma \mathrm{L}_{1}\left(p^{n}\right) \times \Gamma \mathrm{L}_{1}\left(p^{n}\right)$. Since $G_{0}$ is transitive on $V_{1}^{*} \cup V_{2}^{*}$, it follows that $G_{0}^{1}$ and $G_{0}^{2}$ are transitive on $V_{n}(p)^{*}$. By Lemma $17.1,-1 \in G_{0}^{1}$ and $-1 \in G_{0}^{2}$, so (6) holds.

Now suppose for a contradiction that $n=1$. Then $G_{0}^{1}=\mathrm{GL}_{1}(p)=G_{0}^{2}$. For $i \in\{1,2\}$, let $K_{i}$ be the kernel of the projection map of $G_{0, V_{1}}=G_{0} \cap\left(\mathrm{GL}_{1}(p) \times \mathrm{GL}_{1}(p)\right)$ onto $G_{0}^{i}$. Since $G_{0}=G_{0, V_{1}}\langle(t, s) \tau\rangle$ for some $t, s \in \mathrm{GL}_{1}(p)$, there exists $H \unlhd G_{0}^{1}$ such that $K_{1}=1 \times H$ and $K_{2}=H \times 1$, and $\left|G_{0}\right|=2\left|G_{0}^{1}\right||H|$. By assumption, $G_{0}$ is transitive on $V_{1}(p)^{*} \times V_{1}(p)^{*}$, so $(p-1)^{2}$ divides $\left|G_{0}\right|$. Thus $|H|=p-1$ or $(p-1) / 2$, so $\left\langle\zeta^{2}\right\rangle \leqslant H$, where $\zeta:=\zeta_{p}$. Since $\zeta \in G_{0}^{1}=G_{0}^{2}$, it follows that $(\zeta, \zeta) \in G_{0}$, but then $-1 \in G_{0}$, a contradiction.

\section{Example 1.2 And $2-(v, k, 1)$ Designs}

In this section, we consider when a partial linear space from Example 1.2 can be obtained from a 2- $(v, k, 1)$ design using a rank 3 group (in the sense of Remark 1.5 and Lemma 2.11).

Let $V:=U \otimes W$ where $U:=V_{2}(q), W:=V_{m}(q), m \geqslant 2$, and $q$ is a prime power. Let $\mathcal{S}_{U}:=\left(V, \mathcal{L}_{U}\right)$ where $\mathcal{L}_{U}:=\left\{(U \otimes w)+v: w \in W^{*}, v \in V\right\}$, and define $\mathcal{S}_{W}$ and $\mathcal{L}_{W}$ similarly. Recall that $\mathcal{S}_{U} \simeq \mathcal{S}_{W}$ when $m=2$ by Lemma 10.2 (iv). Let $K:=\mathbb{F}_{q^{2}}$. In $\S 11$, we saw that $\mathcal{S}_{U}$ may be viewed as a $K$-dependent proper partial linear space from Example 1.1 with respect to the rank 3 affine primitive group $G:=V:\left(\mathrm{GL}_{m}(q) \circ K^{*}\right)$ : Aut $(K)$ from class (S1). We may define $\mathcal{L}_{Y}:=\left\{\langle u\rangle_{K}+v: u \in Y, v \in V\right\}$, where $Y$ is the orbit of $G_{0}$ on $V^{*}$ consisting of those vectors that are not collinear with 0 in $\mathcal{S}_{U}$, in which case $\mathcal{S}_{Y}:=\left(V, \mathcal{L}_{Y}\right)$ is a $K$-dependent $G$-affine proper partial linear space whose collinearity relation is disjoint from that of $\mathcal{S}_{U}$. Observe that the incidence structure $\left(V, \mathcal{L}_{U} \cup \mathcal{L}_{Y}\right)$ is the linear space $\mathrm{AG}_{m}\left(q^{2}\right)$. Thus the partial linear space $\mathcal{S}_{U}$ can be obtained from a $2-\left(q^{2 m}, q^{2}, 1\right)$ design using a rank 3 group (in the sense of Remark 1.5 and Lemma 2.11). Similarly, in $\S 12$ we saw that for $K:=\mathbb{F}_{q^{3}}$ and $m=3, \mathcal{S}_{W}$ may be viewed as a $K$-dependent proper partial linear space from Example 1.1 with respect to the rank 3 affine primitive group $G:=V:\left(\mathrm{GL}_{2}(q) \circ K^{*}\right)$ : Aut $(K)$ from class $(\mathrm{S} 0)$, in which case $\mathcal{S}_{W}$ can be obtained from the $2-\left(q^{6}, q^{3}, 1\right)$ design $\mathrm{AG}_{2}\left(q^{3}\right)$ using a rank 3 group. However, when $m \geqslant 4$, it turns out that $\mathcal{S}_{W}$ cannot be obtained from any $2-(v, k, 1)$ design using a rank 3 group unless $(m, q)=(5,2)$, which we now prove.

Proposition 18.1. Let $V:=U \otimes W$ where $U:=V_{2}(q), W:=V_{m}(q), m \geqslant 4$ and $q$ is a prime power. Let $\mathcal{L}_{W}:=\left\{(u \otimes W)+v: u \in U^{*}, v \in V\right\}$. The following are equivalent.

(i) $H$ is a permutation group of rank 3 on $V$ and there is a $2-\left(q^{2 m}, q^{m}, 1\right)$ design $\mathcal{D}:=(V, \mathcal{L})$ such that $H \leqslant \operatorname{Aut}(\mathcal{D})$ and $H$ has two orbits on $\mathcal{L}$, one of which is $\mathcal{L}_{W}$.

(ii) $H=V:\left(\Gamma L_{1}\left(2^{2}\right) \otimes \Gamma L_{1}\left(2^{5}\right)\right) \leqslant \mathrm{A}_{1}\left(2^{10}\right)$ and $(m, q)=(5,2)$.

Proof. Suppose that (i) holds, and let $\mathcal{S}_{W}$ denote the partial linear space $\left(V, \mathcal{L}_{W}\right)$. Note that $H \leqslant \operatorname{Aut}\left(\mathcal{S}_{W}\right)$. By Proposition 10.10, $\operatorname{Aut}\left(\mathcal{S}_{W}\right)=V:\left(\mathrm{GL}_{2}(q) \otimes \mathrm{GL}_{m}(q)\right): \operatorname{Aut}\left(\mathbb{F}_{q}\right)$, so $\operatorname{Aut}\left(\mathcal{S}_{W}\right)$ is primitive on $V$ by Lemma 2.2. Thus $H$ is primitive on $V$ by Lemma 2.9. If $H$ is not affine, 
then by Proposition 2.21, either $H$ is almost simple, or $H$ has subdegrees $2\left(q^{m}-1\right)$ and $\left(q^{m}-1\right)^{2}$. However, the former is not possible by Theorem 2.22 since $H$ is not 2-transitive, and the latter is not possible since $m \geqslant 4$ and $\operatorname{Aut}\left(\mathcal{S}_{W}\right)$ has subdegrees $(q+1)\left(q^{m}-1\right)$ and $q\left(q^{m}-1\right)\left(q^{m-1}-1\right)$. Thus $H$ is affine, so the socles of $H$ and $\operatorname{Aut}\left(\mathcal{S}_{W}\right)$ are equal by [49, Proposition 5.1].

Hence $H$ is an affine permutation group of rank 3 on $V$ and $H_{0}$ stabilises the tensor decomposition of $V$. Since $m \geqslant 4, H$ belongs to one of the classes (R0), (I0) or (T1)-(T5) by Theorem 3.2. Recall the definition of the group $H_{0}^{W}$ from the proof of Theorem 3.2. Now $H_{0}^{W}$ is transitive on the lines of $\mathrm{PG}(W)$ since $H_{0}$ is transitive on the set of non-simple tensors of $V$, so either $H_{0}^{W}$ is 2-transitive on $\mathrm{PG}(W)$, or $H_{0}^{W}=\Gamma L_{1}\left(2^{5}\right)$ with $(m, q)=(5,2)$ [35]. If $H_{0}^{W}=\Gamma \mathrm{L}_{1}\left(2^{5}\right)$ and $(m, q)=(5,2)$, then (ii) holds by a computation in MAGMA, so we assume otherwise. By [12], either $\mathrm{SL}_{m}(q) \unlhd H_{0}^{W}$, or $H_{0}^{W}=A_{7}$ and $(m, q)=(4,2)$. In particular, $H_{0}$ is not soluble, so $H_{0}$ does not belong to (R0) or (I0). Let $\mathcal{L}^{\prime}$ be the orbit of $H$ on $\mathcal{L}$ that is not $\mathcal{L}_{W}$. By Lemma $2.11, \mathcal{S}^{\prime}:=\left(V, \mathcal{L}^{\prime}\right)$ is an $H$-affine proper partial linear space with line-size $q^{m}$ for which $\mathcal{S}^{\prime}(0)$ is the set of non-simple tensors of $V$. If $H_{0}$ belongs to one of the classes (T1)-(T3), then by Proposition $10.1, \mathcal{S}^{\prime}$ is $\mathbb{F}_{q}$-dependent, but then $\mathcal{S}^{\prime}$ has line-size at most $q$, a contradiction. Otherwise, $H_{0}$ belongs to (T4) or (T5), in which case $H_{0}$ belongs to (S2) or (S1), respectively. By Proposition 11.2, $\mathcal{S}^{\prime}$ is isomorphic to an $\mathbb{F}_{q^{2}}$-dependent partial linear space, but then $\mathcal{S}^{\prime}$ has line-size at most $q^{2}$, a contradiction.

Conversely, suppose that (ii) holds. Using MAgma and Lemmas 2.3, 2.7 and 2.11, we verify that (i) holds.

\section{REFERENCES}

[1] Aschbacher, M. Finite group theory. Cambridge University Press, 2000.

[2] Bamberg, J., Betten, A., De Beule, J., Cara, P., Lavrauw, M., and NeUNHÖFFER, M. FinInG - a GAP package, Version 1.0, 2013. http://cage.ugent.be/ geometry/fining.php.

[3] Biliotti, M., And Johnson, N. L. The non-solvable rank 3 affine planes. J. Combin. Theory, Ser. A 93 (2001), 201-230.

[4] Biliotti, M., Montinaro, A., And Francot, E. 2- $(v, k, 1)$ Designs with a pointprimitive rank 3 automorphism group of affine type. Des. Codes Cryptogr. 76 (2015), $135-171$.

[5] Blackburn, N., and Huppert, B. Finite groups II. Springer Verlag, Berlin, 1981.

[6] Bosma, W., Cannon, J., And Playoust, C. The Magma algebra system. I. The user language. J. Symbolic Comput. 24 (1997), 235-265.

[7] Bray, J. N., Holt, D. F., And Roney-Dougal, C. M. The maximal subgroups of the low-dimensional finite classical groups. Cambridge University Press, Cambridge, 2013.

[8] Brouwer, A. E., Cohen, A. M., And Neumaier, A. Distance-regular graphs. SpringerVerlag, Berlin, 1989.

[9] Buekenhout, F., Delandtsheer, A., Doyen, J., Kleidman, P. B., Liebeck, M. W., AND SAXL, J. Linear spaces with flag-transitive automorphism groups. Geom. Dedic. 36 (1990), 89-94.

[10] Buekenhout, F., And Van Maldeghem, H. A characterization of some rank 2 incidence geometries by their automorphism group. Mitt. Mathem. Sem. Giessen 218 (1994), 1-70. https://cage. ugent.be/ hvm/Research.html.

[11] Buekenhout, F., and Van Maldeghem, H. Finite distance-transitive generalized polygons. Geom. Dedic. 52 (1994), 41-51.

[12] Cameron, P. J., And Kantor, W. M. 2-Transitive and antiflag transitive collineation groups of finite projective spaces. J. Algebra 60 (1979), 384-422.

[13] Chevalley, C. The algebraic theory of spinors and Clifford algebras: collected works, vol. 2. Springer, Berlin, 1996. 
[14] Conway, J. H., Curtis, R. T., Norton, S. P., Parker, R. A., And Wilson, R. A. Atlas of finite groups. Clarendon Press, Oxford, 1985.

[15] Dembowski, P. Finite geometries, reprint of the 1968 edition. Springer-Verlag, Berlin, 1997.

[16] Devillers, A. A classification of finite partial linear spaces with a primitive rank 3 automorphism group of almost simple type. Innov. Incidence Geom. 2 (2005), 129-175.

[17] Devillers, A. A classification of finite partial linear spaces with a primitive rank 3 automorphism group of grid type. Eur. J. Combin. 29 (2008), 268-272.

[18] Devillers, A., And Hall, J. I. Rank 3 Latin square designs. J. Combin. Theory, Ser. A 113 (2006), 894-902.

[19] Dickson, L. E. Linear groups with an exposition of the Galois field theory. B.G. Teubner, Dresden, 1901.

[20] Dixon, J. D., And Mortimer, B. Permutation groups. Springer Verlag, New York, 1996.

[21] Dye, R. H. Partitions and their stabilizers for line complexes and quadrics. Ann. Mat. Pura Appl. 114 (1977), 173-194.

[22] Fawcett, J. B. Bases of primitive permutation groups. PhD thesis, University of Cambridge, Cambridge, 2013.

[23] Foulser, D. A. The flag-transitive collineation groups of the finite Desarguesian affine planes. Canad. J. Math. 16 (1964), 443-472.

[24] Foulser, D. A. Solvable primitive permutation groups of low rank. Trans. Amer. Math. Soc. 143 (1969), 1-54.

[25] Foulser, D. A., And Kallaher, M. J. Solvable, flag-transitive, rank 3 collineation groups. Geom. Dedic. 7 (1978), 111-130.

[26] The GAP Group. GAP - Groups, Algorithms, and Programming, Version 4.7.5, 2014. http://www.gap-system.org.

[27] Guralnick, R. M. Subgroups of prime power index in a simple group. J. Algebra 81 (1983), 304-311.

[28] Hering, C. Transitive linear groups and linear groups which contain irreducible subgroups of prime order, II. J. Algebra 93 (1985), 151-164.

[29] Hering, C. Two new sporadic doubly transitive linear spaces. In Finite Geometries (1985), C. A. Baker and L. M. Batten, Eds., vol. 103, Dekker, New York, pp. 127-129.

[30] Huppert, B. Zweifach transitive auflösbare Permutationsgruppen. Math. Z. 68 (1957), $126-150$.

[31] Huppert, B. Endliche Gruppen I. Springer-Verlag, Berlin, 1967.

[32] IsaAcs, I. M. Character theory of finite groups. Dover publications, New York, 1994.

[33] Jansen, C., Lux, K., Parker, R., And Wilson, R. An atlas of Brauer characters. Clarendon Press, Oxford, 1995.

[34] Jones, G. A. Paley and the Paley graphs. In Isomorphisms, Symmetry and Computations in Algebraic Graph Theory (2020), G. A. Jones, I. Ponomarenko, and J. Širáň, Eds., vol. 305, Springer, Switzerland, pp. 155-183.

[35] Kantor, W. M. Line-transitive collineation groups of finite projective spaces. Israel J. Math. 14 (1973), 229-235.

[36] Kantor, W. M. Homogeneous designs and geometric lattices. J. Combin. Theory, Ser. A 38 (1985), 66-74.

[37] Kleidman, P., And Liebeck, M. The subgroup structure of the finite classical groups. Cambridge University Press, Cambridge, 1990.

[38] Korchmáros, G. A translation plane of order 49 with non-solvable collineation group. $J$. Geom. 24 (1985), 18-30.

[39] Liebeck, M. W. The affine permutation groups of rank three. Proc. London Math. Soc 54 (1987), 477-516.

[40] Liebeck, M. W. The classification of finite linear spaces with flag-transitive automorphism 
groups of affine type. J. Combin. Theory, Ser. A 84 (1998), 196-235.

[41] Liebeck, M. W., Praeger, C. E., and Saxl, J. On the O'Nan-Scott theorem for finite primitive permutation groups. J. Austral. Math. Soc. 44 (1988), 389-396.

[42] Liebeck, M. W., And SaxL, J. The finite primitive permutation groups of rank three. Bull. London Math. Soc. 18 (1986), 165-172.

[43] Lüneburg, H. Translation planes. Springer-Verlag, Berlin, 1980.

[44] Mason, G., And Ostrom, T. G. Some translation planes of order $p^{2}$ and of extra-special type. Geom. Dedic. 17 (1985), 307-322.

[45] McKay, B. D., And Piperno, A. Practical graph isomorphism, II. J. Symbolic Computation 60, 94-112.

[46] Montinaro, A. 2- $(v, k, 1)$ Designs admitting a primitive rank 3 automorphism group of affine type: the extraspecial and the exceptional classes. J. Combin. Designs 23 (2015), 481-498.

[47] Ostrom, T. G. Elementary abelian 2-groups on the line at infinity of translation planes. J. Geom. 17 (1981), 128-139.

[48] Pearce, G., and Praeger, C. E. Rank 3 transitive decompositions of complete multipartite graphs. Graphs Comb. 29 (2013), 669-680.

[49] Praeger, C. E. The inclusion problem for finite primitive permutation groups. Proc. London Math. Soc. 60 (1990), 68-88.

[50] Praeger, C. E., And Saxl, J. Closures of finite primitive permutation groups. Bull. London Math. Soc. 24 (1992), 251-258.

[51] Shult, E., AND Surowski, D. Algebra: a teaching and source book. Springer, Cham, 2015.

[52] SoICher, L. H. Grape - a GAP package, Version 4.6.1, 2012. https://gap-packages. github.io/grape/.

[53] Suzuki, M. On a class of doubly transitive groups. Ann. Math. (1962), 105-145.

[54] Walker, M. A characterization of some translation planes. Abh. Math. Sem. Univ. Hamb. 49 (1979), 216-233.

[55] Wilson, R. A. The finite simple groups. Springer, London, 2009.

[56] Wilson, R. A., ET AL. AtLAS of finite group representations. http://brauer.maths. qmul.ac.uk/Atlas/v3.

[57] Zassenhaus, H. Über endliche Fastkörper. Abh. Math. Sem. Univ. Hamb. 11 (1935), $187-220$.

[58] Zsigmondy, K. Zur Theorie der Potenzreste. Monatsh. Math. Phys. 3 (1892), 265-284.

(Bamberg, Devillers, Praeger) Centre for the Mathematics of Symmetry and Computation, Department of Mathematics and Statistics, The University of Western Australia, 35 Stirling Highway, Crawley, W.A. 6009, Australia

Email address: john.bamberg@uwa.edu.au, alice.devillers@uwa.edu.au, cheryl.praeger@uwa.edu.au

(Fawcett) Department of Mathematics, Imperial College London, South Kensington Campus, LonDON, SW7 2AZ, United Kingdom

Email address: j.fawcett@imperial.ac.uk 